\title{
Nanoscale organization and dynamics of SNARE proteins in the presynaptic membranes
}

\author{
Dissertation \\ for the award of the degree \\ "Doctor rerum naturalium" \\ of the Georg-August-Universität Göttingen \\ within the doctoral program Molecular Biology \\ of the Georg-August University School of Sciences (GAUSS)
}

submitted by

Dragomir Milovanović

from Belgrade

Göttingen, 2015 
Thesis Advisory Committee:

Prof. Dr. Reinhard Jahn

Department of Neurobiology, Max Planck Institute for Biophysical Chemistry

Prof. Dr. Stefan W. Hell

Department of NanoBiophotonics, Max Planck Institute for Biophysical Chemistry

Prof. Dr. Andreas Janshoff

Institute of Physical Chemistry, University of Göttingen

Ass. Prof. Dr. Geert van den Bogaart

Department of Tumor Immunology, Radboud University Medical Center

Extended Evaluation Committee (alphabetically):

Prof. Dr. Ulf Diederichsen

Institute of Organic and Biomolecular Chemistry, University of Göttingen

Prof. Dr. Stefan Jakobs

Department of Neurology, University of Göttingen Medical Center

Prof. Dr. Claudia Steinem

Institute of Organic and Biomolecular Chemistry, University of Göttingen

Oral examination: October $5^{\text {th }}, 2015$ 


\section{TABLE OF CONTENTS}

Acknowledgements .............................................................

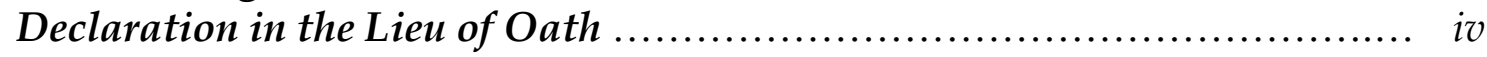

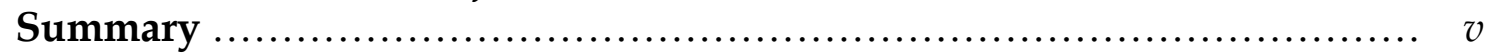

\section{Introduction}

1.1 Biochemical properties of the lipid bilayer ............................ 3

1.1.1 Composition of the lipid bilayer ...................................... 3

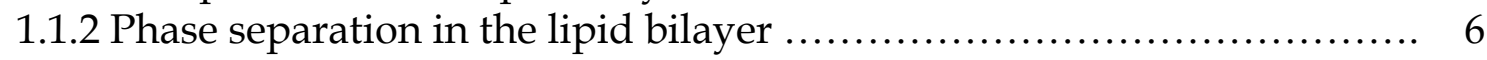

1.1.3 Cholesterol affects the lipid bilayer properties ........................ 9

1.2 Characteristics of the cellular membranes ............................... 10

1.2.1 Cellular membranes are rich in different protein and lipid species ....... 10

1.2.2 Proteins and lipids generate membrane curvature ...................... 12

1.2.3 Lipid asymmetry in cellular membranes ............................ 15

1.2.4 Phase separation in cellular membranes .............................. 16

1.2.5 Cytoskeleton and scaffolding proteins fine-tune the plasma membrane structure ..................................................................... 19

1.3 SNAREs as tools to understand the physical principles behind membrane

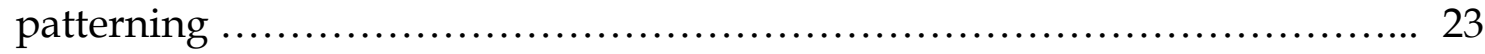

1.3.1 Segregation within the hydrophobic core of the membrane ............. 24 1.3.2 Segregation caused by interactions at the hydrophobic-hydrophilic

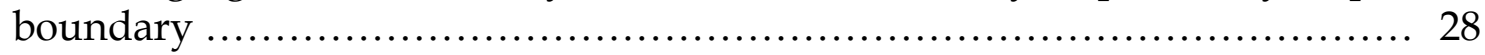

1.3.3 Segregation due to interactions in the hydrophilic space $\ldots \ldots \ldots \ldots \ldots \ldots \ldots . . .30$

1.4 Functional relevance of SNARE clustering ........................... 31

1.5 Aims of this Thesis ................................................... 35

\section{Material and Methods}

2.1 Peptides and lipids ..................................................... 38

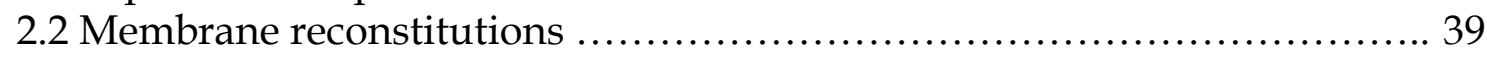

2.2.1 Polymer supported membranes ........................................ 39

2.2.2 Stacked lipid bilayers ............................................ 40

2.2.3 Large unilamellar vesicles .............................................. 41

2.2.4 Giant unilamellar vesicles ......................................... 42

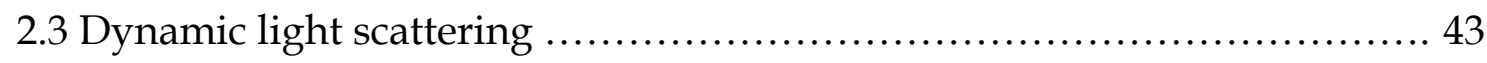


2.4 Cell culture and immunofluorescence ................................. 44

2.5 Superresolution stimulated emission depletion (STED) nanoscopy ........ 46

2.6 Fluorescence correlation spectroscopy ................................. 50

2.7 Förster resonance energy transfer .................................... 52

\section{Results}

3.1 Hydrophobic mismatch between the lipid bilayer and the transmembrane domains drives SNARE clustering ......................................... 55 3.2 Hydrophobic mismatch shapes syntaxin clusters together with the surface ionic interactions and specific protein-protein interactions . .64 3.3 Calcium acts as a charge bridge that connects multiple syntaxin $1 / \mathrm{PI}(4,5) \mathrm{P}_{2}$ clusters into larger mesoscale domains 72

\section{Discussion}

4.1 Difference between membrane thickness and the length of the TMDs can drive clustering of membrane proteins ...................................... 81 4.2 Cholesterol increases membrane thickness and thereby increases hydrophobic mismatch

4.3 Electrostatic interactions, protein-protein interactions and hydrophobic mismatch all modulate lateral organization of SNAREs ..................... 84 $4.4 \mathrm{Ca}^{2+}$ acts as a charge bridge that connects multiple syntaxin $1 / \mathrm{PI}(4,5) \mathrm{P}_{2}$ clusters into larger mesoscale domains ...................................... 87 4.5 Physiological significance of SNARE clustering in the plasma membrane 88

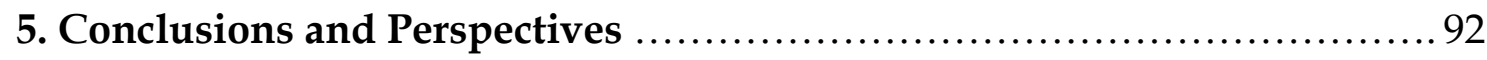

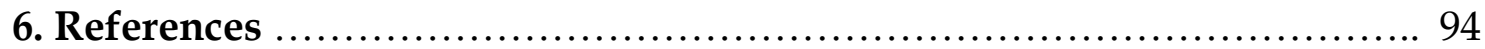

\section{Appendices}

7.1 Information on peptide synthesis .................................... 117

7.2 Information on imaging ellipsometry ............................... 118

7.3 Information on atomic force microscopy measurements .................. 119

8. List of Figures ................................................... 120

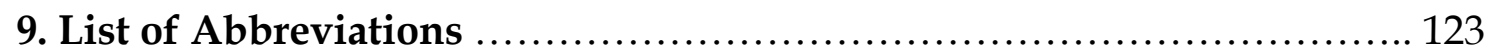

10. Curriculum vitae with the List of Publications .........................125 


\section{ACKNOWLEDGEMENTS}

These wonderful years in Göttingen are marked by many diverse personalities that were source of my motivation and inspiration. I am in debt to all people who shaped my professional and personal time and here I specially thank to:

- $\quad$ Reinhard Jahn for the curiosity driven research and for being a true mentor who took care of my comprehensive professional development.

- Geert van den Bogaart for his engagement, support and many great hours we have spent together in the lab.

- $\quad$ Stefan W. Hell for the support and freedom to use different, exciting setups while tackling my biological questions. Overall, the Department of NanoBiophotonics is an amazing blend of physicists, chemists and biologists, and I enjoyed learning from all of them.

- Alf Honigmann for teaching me how to use the setups and sharing my daily excitements while doing the experiments. Also, to Fabian Göttfert with whom I enjoyed discussing different setups.

- Andreas Janshoff for his input during our vivid discussions and suggestions for experiments that strengthened this work.

- $\quad$ Ulf Diederichsen for contributing with the expertise in peptide synthesis that was important for many experiments performed within this work.

- $\quad$ Claudia Steinem for her immense engagement in providing the stimulating platform for people passionate about the biological membranes.

- Iwan Schaap and Mitja Platen for our great venture into atomic force microscopy of biomembranes.

- Helmut Grubmüller and Herre Jelger Risselada for inspiring discussions and modeling of domain formation.

- $\quad$ Stefan Jakobs for taking part in the evaluation of this work.

- My colleagues from the Department of Neurobiology for the wonderful atmosphere and stimulating discussions. Particularly to my dear students: Nathan Pagano, Vedran Vasić and Sebastian Sydlik, all of whom I worked with pleasure.

- $\quad$ Scientists whose input I always highly valued: Christian Griesinger, Christian Eggeling, Silvio Rizzoli, Lukas Tamm, Aki Kusumi, Gerhard Schütz, Mary Osborne, Vesna Niketic, Ratko Jankov, Stephan Ferneding.

- My MolBio Family, especially my fellows from the class 2011/2012: it was such a remarkable year! Also, to Steffen Burkhardt and Kerstin Grüniger for taking care that all the elements run smoothly; and to colleagues from the GGNB Office for the nice time during my term as a student representative.

- Without listing, I am thankful to all my Family and Friends, especially to my parents Petra \& Dragan, and to Christian, for immense support and making the time of graduate studies unforgettable and my life emotionally fulfilled! 


\section{Declaration in the Lieu of Oath}

Herewith I declare, that I prepared the Doctoral Thesis:

Nanoscale organization and dynamics of SNARE proteins in the presynaptic membrane

on my own and with no other sources and aids than quoted.

Göttingen, August 7th 2015

Dragomir Milovanovic 


\section{SUMMARY}

The specific organization of proteins and lipids in functional domains in biological membranes allows localization and segregation of specific physiological activities. Mechanisms that underlie the formation of these domains include hydrophobic and ionic interactions with membrane lipids as well as specific protein-protein interactions.

Using plasma membrane-resident SNARE proteins as a model, I show that cholesterol-induced hydrophobic mismatch between the transmembrane domains and the membrane lipids not only suffices to induce clustering of proteins, but can also lead to the segregation of structurally closely homologous membrane proteins in distinct membrane domains. Domain formation is further fine-tuned by interactions with polyanionic phosphoinositides and proteins. Furthermore, $\mathrm{Ca}^{2+}$ acts as a charge bridge that connects multiple syntaxin $1 / \mathrm{PI}(4,5) \mathrm{P}_{2}$ complexes into larger domains.

Segregating SNARE proteins into distinct clusters at the plasma membrane has three key functional implications for exocytosis: (i) clusters act as the local hot spots for the vesicle recruitment, (ii) the local enrichment provides sufficient number of proteins necessary for the fast, evoked synaptic release, (iii) closely homologous SNARE proteins such as syntaxin 1 and 4 are segregated in non-overlapping membrane domains which is essential for their distinct roles in regulated (syntaxin 1) and constitutive (syntaxin 4) exocytosis.

Overall, the findings presented in this thesis demonstrate that the structural organization of membranes is governed by a hierarchy of interactions with hydrophobic mismatch emerging as one of the fundamental principles. 



\section{INTRODUCTION}

Early concepts on the plasma membrane structure date back to the beginning of twentieth century when Langmuir described the molecular nature of a lipid monolayer spread at the air/water interface as a result of lipids containing both hydrophobic regions (acyl chains) oriented towards the air and polar (head-groups) facing the aqueous environment (Langmuir, 1917). His assay pioneered by Agnes Pockels in Braunschweig, to later be adapted to what is now known as the Langmuir-Blodgett assay, helped to further characterize the properties of the lipid bilayer (Blodgett, 1935; Pockels, 1891; 1894; Rayleigh, 1899). Using similar assay, Gorter and Grendel compared the surface occupied by lipids extracted from erythrocytes with the average size of erythrocyte and they concluded that the naturally occurring membranes are composed of the lipid bilayer (Gorter \& Grendel, 1925).

The saga of membrane research kept evolving, especially with the development of the electron microscope. Initial micrographs where the lipid bilayer was lightly stained in contrast to aqueous boundary led to the concept of tri-striata (three layer) organization of the plasma membrane. Here, the lipid bilayer sandwiched with the proteins at both cytosolic and extracellular facets (Danielli \& Davson, 1935; Robertson, 1960; 1963). However, further studies of the hydrophobic interactions between proteins and lipids (Lenard \& Singer, 1966; Wallach \& Zahler, 1966) indicated that the plasma membrane is not fully covered by proteins, but rather contains proteins that either span the entire membrane (integral) or are embedded in the single monolayer (peripheral). Also, these proteins were shown to undergo lateral diffusion in the membrane (Frye \& Edidin, 1970). Altogether this has led to the refinement of the plasma membrane organization into the so-called fluid-mosaic model. In this model, the plasma membrane is a dynamic structure in which all components are able to diffuse laterally, with the membrane proteins "floating like icebergs in a sea" of membrane lipids (Singer \& Nicolson, 1972). 
Although the fluid-mosaic model still forms the foundation of our present understanding of the biological membranes, it is becoming evident that lipids and proteins form subdomains within the membrane whose properties are slowly emerging (Fig. 1.1). Here I start by describing the general properties of the lipid bilayers (Chapter 1.1). Biophysical approaches and membrane reconstitutions substantially contributed to membrane biology and I present some of the key parameters that underlie the complexity of cellular membranes (Chapter 1.2). Particularly the soluble NSF-attached protein receptor (SNARE) family of proteins has served as an excellent paradigm to look at the mechanisms that shape the structure of the plasma membrane and these major mechanisms are presented in Chapter 1.3. Using SNAREs as an example, in Chapter 1.4 I present the functional significance of protein/lipid domains in the plasma membrane. This also leads us to the Aims and the main hypothesis of this Thesis (Chapter 1.5).
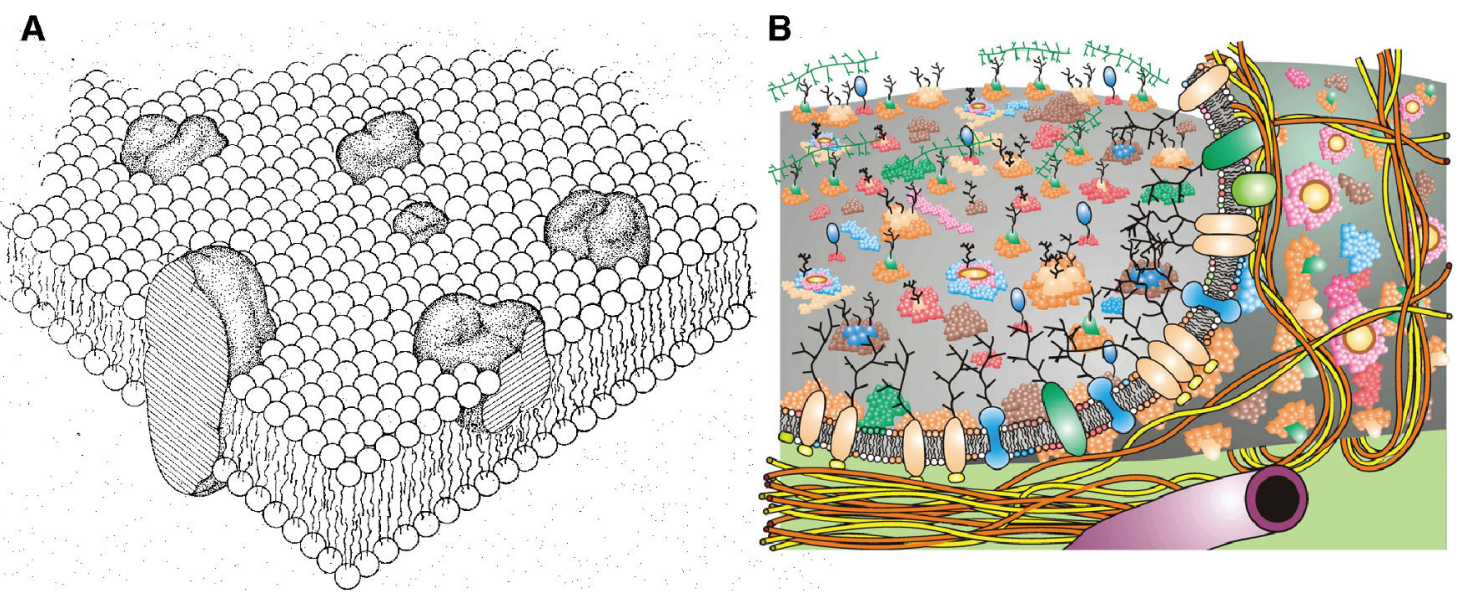

Figure 1.1. Evolving view on the plasma membrane organization. A. The scheme of the initial fluid-mosaic model of membrane organization. The membrane contains proteins that span both layers (integral) or are embedded in a single layer (peripheral). B. Evolved view on the plasma membrane structure demonstrates multiple protein/lipid domains as well as the underlying cytoskeleton. Adapted from Nicolson, 2014; Singer \& Nicolson, 1972. 


\subsection{BIOCHEMICAL PROPERTIES OF THE LIPID BILAYER}

\subsubsection{Composition of the lipid bilayer}

The lipid bilayer is composed of two layers of glycerophospholipids which acyl chains are oriented toward each other generating the hydrophobic core and polar head groups that are exposed to the aqueous environment (Nagle \& Tristram-Nagle, 2000; Zaccai, Blasie, \& Schoenborn, 1975). The backbone of glycerophospholipids is made of the alcohol glycerol where fatty acids are esterified at positions 1 and 2 and at position 3 a polar head-group is attached vis a phosphate group (e.g. choline, ethanolamine, serine). The close association of phospholipids is mediated by non-covalent, hydrophobic interactions (Bagatolli, Ipsen, Simonsen, \& Mouritsen, 2010). The stability of the simple phospholipid bilayer is generally determined by three parameters: (i) repulsion between the neighboring head-groups, (ii) attractive interaction of neighboring lipids to minimize the contact area between the hydrophobic tails and the surrounding water molecules and (iii) repulsion of neighboring lipids due to collisions of flexible acyl chains. The pressure along the bilayer (Fig. 1.2) can be disrupted by different lipids (i.e. sterols) or proteins, which can lead to changes in membrane shape and make the bilayer more or less prone to remodeling (Bagatolli et al., 2010; Kozlov et al., 2014).

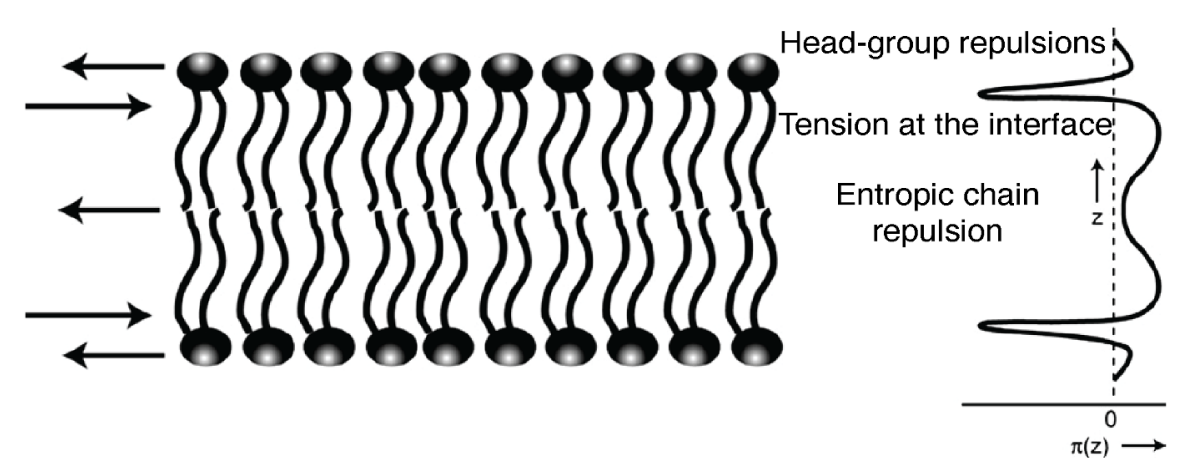

Figure 1.2. Scheme of the lipid bilayer. The contribution of individual region of the lipid bilayer to the lateral pressure is indicated. Adapted from Bagatolli et al., 2010. 
Apart from phospholipids, another family of lipids often found in membranes is the sphingolipids. In contrast to glycerophospholipids, sphingolipids contain the 18-C amino alcohol with a trans double bond as a backbone. Attachment of a fatty acid to amino group generates ceramide and further attachment of polar (phospho)head-group (i.e. ethanolamine, PE; choline, PC) gives rise to sphingomyelin (SM). Sphingomyelin is the most abundant sphingolipid and accounts for an average of $20 \mathrm{~mol} \%$ of total plasma membrane lipids.

Both acyl chains and the head-groups of phospholipids can vary in their structure. Acyl chains can either be completely saturated alkyls or they can contain one or more double bonds (mono- and polyunsaturated lipids). The existence of even a single double bond can alter the bilayer (Feller, 2008). The double bond is more polar than a single $\mathrm{C}-\mathrm{C}$ bond and it also introduces a change in the conformational freedom of the acyl chain. The saturated acyl chains are in all trans form. However, a double bond induces a gauche conformation and one gauche conformation $\left(120^{\circ}\right)$ is followed by another gauche conformation $\left(-120^{\circ}\right)$ of either of the two neighboring bonds

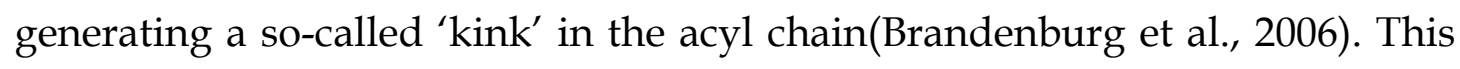
directly affects the spatial packing of the acyl chain by increasing the area occupied per lipid (Fig. 1.3; Niemelä, Hyvönen, \& Vattulainen, 2006). On the other hand, saturation increases the thickness of the lipid bilayer due to tight hydrophobic packing of the acyl chains (Li, Vorobyov, \& Allen, 2012). 

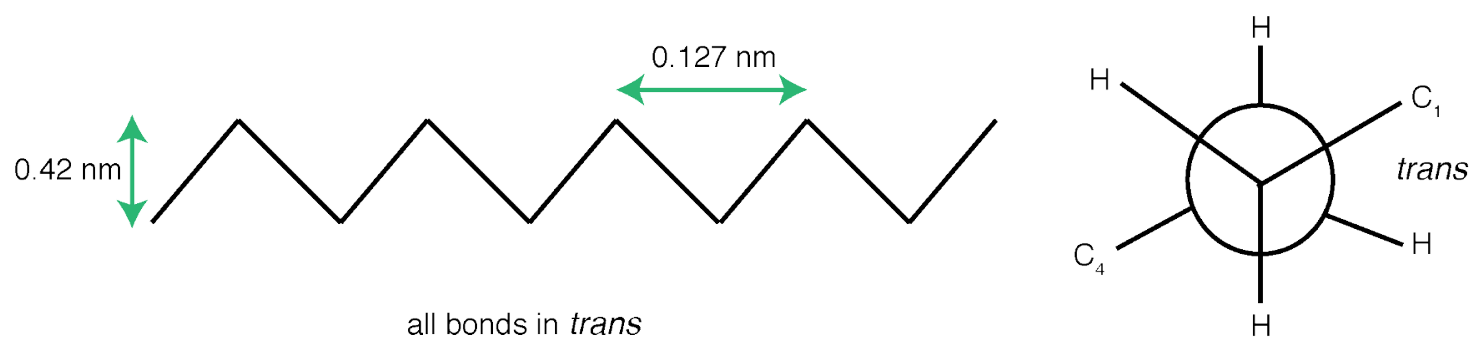

all bonds in trans

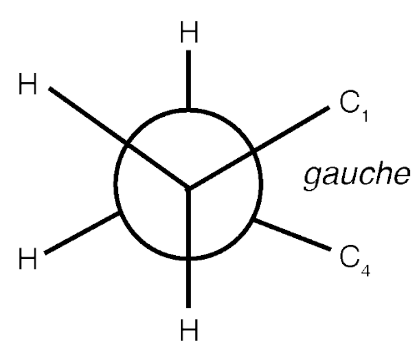

double gauche generates kink in the acyl chain

Figure 1.3. Schemes and Newman projections of acyl-chains with all bonds in trans (top) and mixture with one cis bond (bottom). Even a single cis bond causes two subsequent gauche conformations, which results in a kink within the acyl chain. Adapted from Brandenburg et al., 2006.

Head-groups are always polar, but their charge can vary (Fig. 1.4). Phosphatidyl-choline (PC) and phosphatidyl-ethanolamine (PE) head-groups have their pKa around 14 and 8, respectively. This implies that in the cell ( $\mathrm{pH}$ 7.35) these head-groups will be positively charged. However, due to the negative charge of the phosphate group in the neck region of the phospholipid the overall charge of these phospholipids will be zero. Phosphatidyl-serine (PS) contains both ternary amino group (pKa 9) and the carboxyl group (pKa 3); this together with the phosphate group from the neck region makes phosphatidyl-serine derivatives carrying a single negative charge at physiological $\mathrm{pH}$. The most interesting are the lipids with the phosphoinositide head-groups that can be mono- and polyphosphorylated at the 3', 4' and 5' positions (McLaughlin \& Murray, 2005; McLaughlin, Wang, Gambhir, \& Murray, 2002). The charge of these lipids varies in the range between -3 and -7 at physiological $\mathrm{pH}$, making these lipids the main modulators of the bilayer electrostatics. 


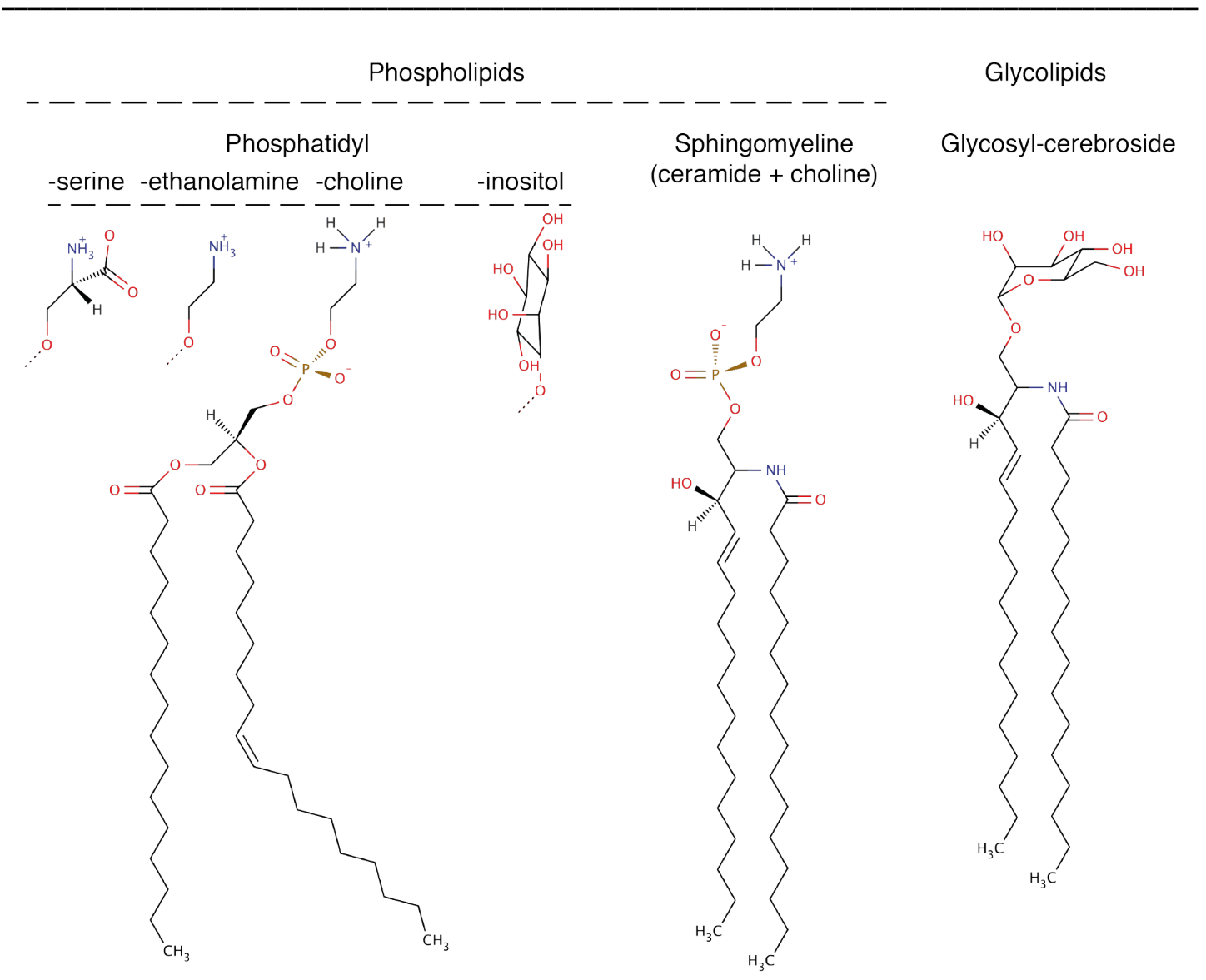

Figure 1.4. Formulas of the major lipid classes. Charge of the polar headgroups at $\mathrm{pH} 7.4$ is indicated.

\subsubsection{Phase separation in the lipid bilayer}

Phase transition is the change of a substance from one thermodynamic state to another and it mostly refers to the change between solid, liquid or gaseous states (Fig. 1.5; Atkins \& de Paula, 2014). All the elements and substances can transition from one phase to another at the specific temperature and pressure. By the general Gibbs phase rule, the total number of coexisting phases in a system of fixed thermodynamic variables is determined by the number of components in the system. If we assume a onecomponent system, a single phase would be present if there is no variation in pressure and temperature. At the phase boundary, two phases coexists and in the case of zero degrees of freedom we would have a single, triple-point (coexistence of three phases; Atkins \& de Paula, 2014). 
Under constant pressure, the lipid bilayer can undergo three distinct phases depending on the temperature (Fig 1.6A). Each of these membrane phases has a characteristic local order (chain conformation) and crystallinity (two dimensional deposition of molecules on the membrane plane). The first is the gel phase, also called solid ordered phase (So). In So phase lipids are mostly ordered in all-trans and arranged in two dimensional triangular lattice. The diffusion of lipids is very slow in this phase $\left(\sim 0.5 \mu \mathrm{m}^{2} \mathrm{~s}^{-1}\right.$; Bacia et al., 2004). The second phase is the liquid ordered phase (Lo, also called ripple phase). The Lo phase is a partially melted lipid phase with a lower average degree of chain ordering than in the So phase. The diffusion of lipids is faster $\left(\sim 1 \mu \mathrm{m}^{2} \mathrm{~s}^{-1}\right)$ than in So phase although the acyl chains still have a high order. The third characteristic phase is liquid disordered phase ( $\operatorname{L} \alpha$, also known as fluid phase). In L $\alpha$ phase the acyl chains are mostly disordered and the lipid diffusion is fast $\left(\sim 1 \mu \mathrm{m}^{2} \mathrm{~s}^{-1}\right)$ and acyl chains are disordered.

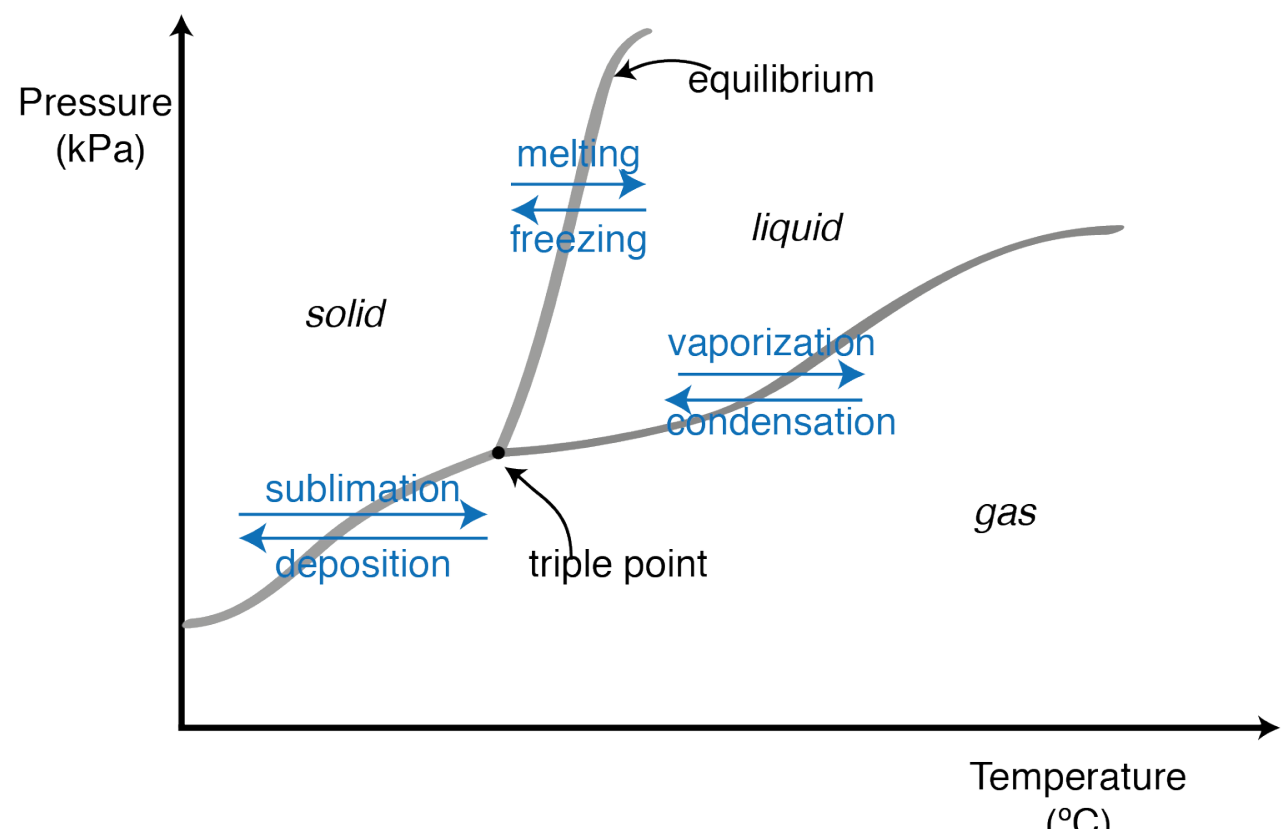

$\left({ }^{\circ} \mathrm{C}\right)$

Figure 1.5. Scheme of the classical phase diagram for a given substance under different pressure and temperature. Gray lines indicate the conditions at which two states of the substance coexist; phase transition occurs as the lines are crossed. Triple point is a condition at which a particular substance coexists in all three phases. 
In the 1980's Tamm and McConnell measured the lateral diffusion coefficients of fluorescently labeled lipids in a reconstituted lipid bilayer (Tamm, 1985; McConnell, Tamm, \& Weis, 1984). Already in this simple monolipid mixture, it was clear that temperature increase changes the phase (Fig. 1.6B). Indeed, apart from the chemistry of lipid bilayer (level of acyl chain saturation, number of components), temperature affects the phase transition of lipid bilayer. As a rule of thumb, fluidity increases with the degree of unsaturation and with the rise of temperature.

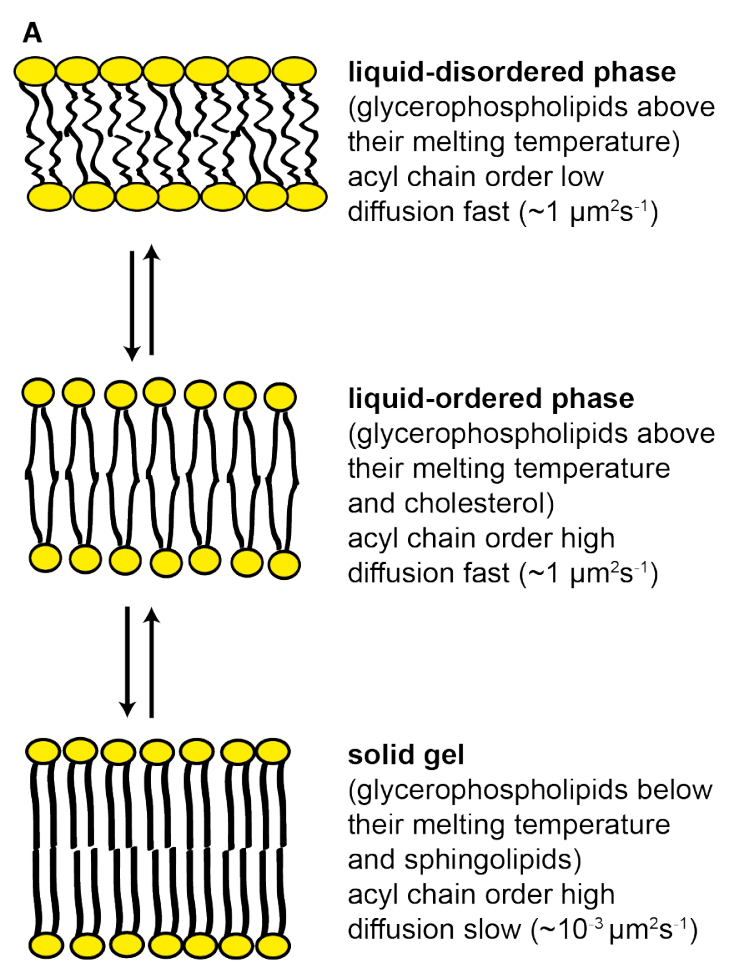

B

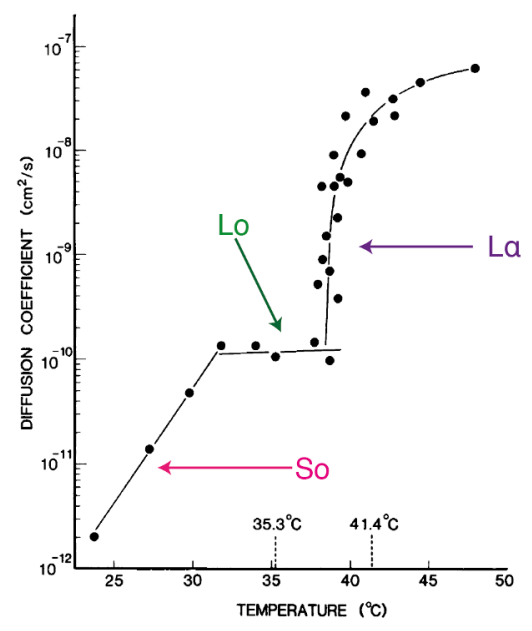

Figure 1.6. Three characteristic phases of the lipid bilayer: solid (So), liquid ordered (Lo) and liquid disordered (L $\alpha$ ) phases. A. Schematic representation of different phases. Lo phase is characterized with a higher order of acyl chain (similar to So) and a fast lateral diffusion (similar to $L \alpha$ ). B. The lateral diffusion coefficient of NBD-PE in DPPC bilayer. Two thermal transitions occur leading to the change from So to Lo phase (at $\sim 32^{\circ}$ ) and from Lo to L $\alpha$ (at $\sim 40^{\circ}$ ). Adapted from Tamm, 1985. 
For a two-component system (binary lipid mixtures), another degree of freedom is opened and a two-phase region can occur. In this case, as the critical melting temperature for a particular lipid membrane is present where the transition from a solid to the more liquid phase occurs (Veatch \& Keller, 2005). For a three component systems the phase diagram can be plotted as a prism (Komura, Shirotori, Olmsted, \& Andelman, 2004). The most commonly used ternary lipid mixtures include cholesterol (C), unsaturated (U) and saturated (D) phospholipids. In this phase prism, for a given temperature we can get the phase-transition triangle with two-phase and three-phase coexisting regions (Fig. 1.7).

A

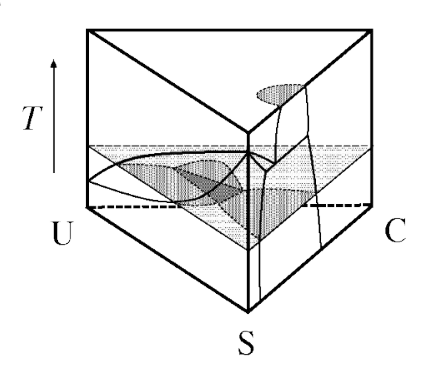

B

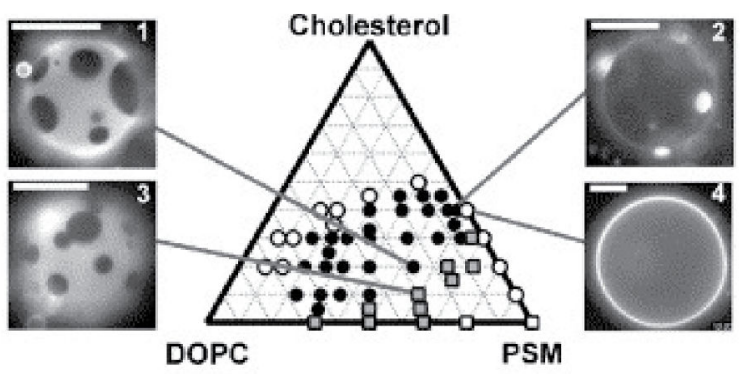

Figure 1.7. Phase transition in model lipid membranes. A. Phase prism of a ternary lipid system consisting of saturated lipid (S), unsaturated lipid (U), and cholesterol (C) at varying temperatures. On the triangle plane for the given temperature we can see light gray (two-phase) and dark gray (threephase) regions. B. Example of a triangle phase plane at $25^{\circ} \mathrm{C}$. Phase separation that was observed in GUVs (DOPC, SM and cholesterol) included uniform phases (white circles/liquid, white squares/solid), coexisting So and Lo phases (gray squares) and Lo and La (black circles). Representative images of GUVs are shown. Adapted from Komura et al., 2004; Veatch \& Keller, 2005.

\subsubsection{Cholesterol affects the lipid bilayer properties}

Cholesterol is a planar, polycyclic compound with a single $3^{\prime}-\mathrm{OH}$ group. The size of cholesterol allows it to span one bilayer in the membrane, with its $\mathrm{OH}$-group oriented towards the aqueous side and the planar polycyclic region intercalating the acyl chains. Interestingly, the effects of cholesterol depend substantially on the chemical nature of the surrounding acyl chains (Lindblom, Orädd, \& Filippov, 2006; Marsh \& Smith, 1973; Reich 
et al., 2008; Rubenstein, Smith, \& McConnell, 1979). In case of lipids with mono- or polyunsaturated acyl chains, cholesterol will increase the thickness of the bilayer, and decrease the area per lipid making the bilayer overall less fluid. For saturated acyl chains, cholesterol will disturb the all-trans arrangements, decreasing the thickness and increasing the area per lipid.

In the context of lipid phases, cholesterol will be the prime component to generate the Lo phase (i.e. it may condense the bilayer; Marsh \& Smith, 1973; Rubenstein et al., 1979; Krause, Daly, Almeida, \& Regen, 2014). In a single-component lipid mixture, the phospholipid head-groups occupy approximately half of the surface area whereas the other half are partially hydrated alkane groups of the acyl chain (Aittoniemi, Niemelä, Hyvönen, Karttunen, \& Vattulainen, 2007). By introducing cholesterol in the membrane, these partially hydrated ('wet') alkane groups are replaced by $-\mathrm{OH}$ group of cholesterol. This increases downstream hydrophobic contact between the acyl chains and strengthens the packing of the bilayer.

\subsection{CHARACTERISTICS OF THE CELLULAR MEMBRANES}

\subsubsection{Cellular membranes are rich in different protein and lipid species}

Lipid synthesis occurs mostly in the ER, Golgi and mitochondria. Three main mechanisms account for the dynamic exchange of lipids between different organelles and the plasma membrane. First, vesicular trafficking along the secretory pathway sorts not only proteins, but also the accompanying lipids from a donor to a target membrane. Second, membrane contact sites, mostly mediated by ER, are zones where lipids can diffuse freely between different membrane compartments. Third, specific classes of soluble, cytosolic proteins are specialized for lipid transfer between the membranes. The best described are oxysterol-binding protein (OSBP) and its related proteins (ORPs). Humans and yeast contain sixteen and seven ORPs, respectively (Schulz \& Prinz, 2007). Structural analyses of yeast ORP - Osh4p indicated that it contains hydrophobic pocket that can accommodate a sterol molecule. Hence, Osh4p has been proposed to bind to cholesterol in ER and exchange it for PI4P in the plasma membrane (Mesmin, Antonny, \& Drin, 2013; Schulz \& Prinz, 2007). Similarly, tricalbins in yeast (E-Syts in 
mammalian cells) constitute a family of tethering proteins that contain a SMP domain, which binds phospholipid acyl-chains and allows their transfer between ER and the plasma membrane (Giordano et al., 2013; Manford, Stefan, Yuan, Macgurn, \& Emr, 2012; Stefan, Manford, \& Emr, 2013).

Also, the presence of specific kinases and phosphatases at particular membranes generates the specific polyphosphoinositide profile along the trafficking path. The best know example is the $\mathrm{PI}(4,5) \mathrm{P}_{2}$ involvement in synaptic vesicle cycle (Di Paolo \& De Camilli, 2006; Di Paolo et al., 2004). $\mathrm{PI}(4,5) \mathrm{P}_{2}$ appears to be enriched in regions where synaptic vesicles dock and eventually fuse (Honigmann et al., 2013; James, Khodthong, Kowalchyk, \& Martin, 2008; Milosevic et al., 2005). In addition downstream $\mathrm{PI}(4,5) \mathrm{P}_{2}$ together with membrane proteins signals for the recruitment of adaptor proteins (i.e. AP2) that will initiate clathrin-formation and membrane engulfment (Jung et al., 2007). Finally, upon the cleavage of the phosphate groups of $\mathrm{PI}(4,5) \mathrm{P}_{2}$ by $4^{\prime}, 5^{\prime}$ phosphatase - synaptojanin 1 , newly endocytosed vesicles release their coat (Jung \& Haucke, 2007). This shows that the lipid cycle is tightly coupled to the protein cycle during exo- and endocytosis (Haucke, Neher, \& Sigrist, 2011).

In addition to a large variety of lipids, the plasma membrane contains thousands of different protein species. It is especially important to note the high protein occupancy of the bilayer volume $(\sim 20 \%)$, as indicated by the analysis of organelles (Takamori et al., 2006) and plasma membranes (Dupuy \& Engelman, 2008). Also, many of the membrane proteins have substantially large cytosolic domains and hence the membrane surface coverage goes between $45-60 \%$ (Wilhelm et al., 2014). Due to such a high abundance and versatility of proteins and lipids, a simple, two-component system such as with a liquid ordered and liquid disordered phase does not provide a satisfactory explanation of membrane structure. Moreover, it seems reasonable to look at the proteins as an integral membrane "phase". Additional factors that affect the structure and dynamics of cellular membranes are discussed below. 


\subsubsection{Proteins and lipids generate membrane curvature}

The relative extension of the polar head-group in relation to the acylchains defines the overall shape of a lipid species (Fig. 1.8A). Small headgroups lead to a cone shaped lipid that favors concave membranes. Similarly, large head-groups lead to inverted cones that favor convex curvature. During membrane bending (Fig. 1.8B) inverted-cone lipids will be enriched at the outer leaflet (positive curvature) and cone lipids at the inner leaflet (negative curvature; Cooke \& Deserno, 2006; Frolov, Shnyrova, \& Zimmerberg, 2011).

A
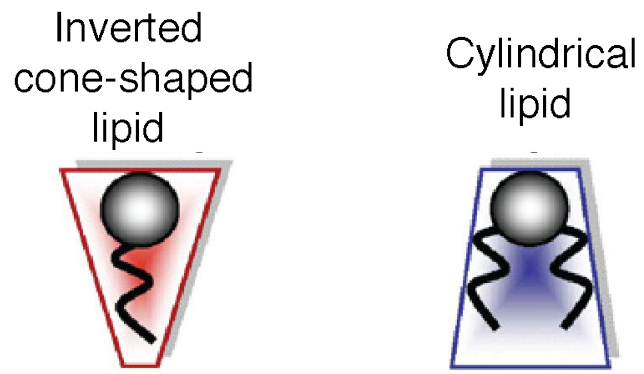

B

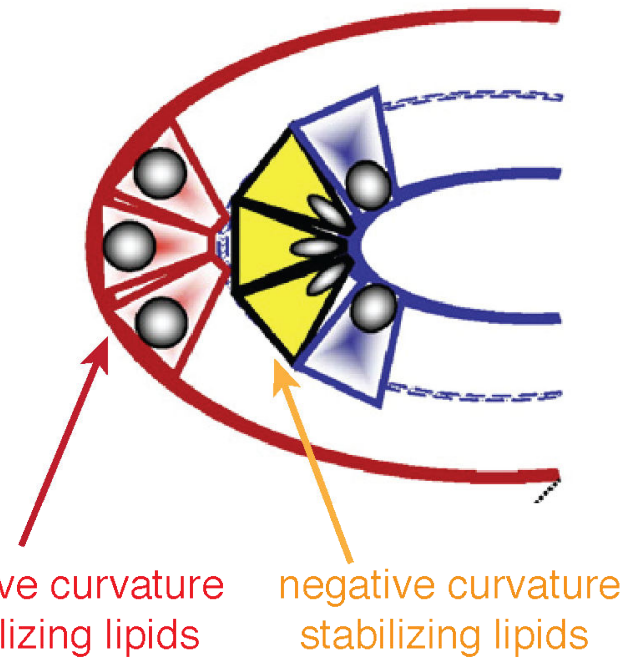

Cone-shaped

lipid

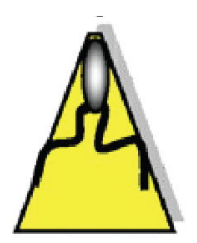

Figure 1.8. Different lipid shapes underlie spontaneous membrane curvature. A. Molecular shape of lipids depends on the area occupied by the polar-head group and acyl chain: inverted cone (polar head-group occupies larger area), cylindrical (similar area occupancy of both head-group and acyl chain) and cone shaped (acyl chain occupies larger area). B. Inverted cone lipids stabilize positive curvature (outer leaflet) and cone-shaped lipids stabilize negative curvature (inner leaflet). Adapted from Boukh-Viner \& Titorenko, 2006. 
In the context of cellular membranes, proteins can enhance or reduce curvature in several ways. Proteins such as clathrin, COPI and COPII coordinate the generation of scaffolds around the membrane regions which generates the vesiculation of membranes. Also, protein crowding in membranes induces local destabilization of the bilayer. Amphipathic protein domains that can locally and asymmetrically insert into the lipid bilayer can further induce or enhance curvature formation (Kirchhausen, 2012).

The main molecular cues that would recruit specific proteins scaffolds on the membrane are lipid packing defects and accumulation of charged lipids (Fig. 1.9; Bigay \& Antonny, 2012). Membranes of the intracellular organelles (ER, cis-Golgi) where most of the lipid synthesis takes place are characterized by loose lipid packing and larger fluidity. This is a consequence of low cholesterol amounts and abundant polyunsaturated acyl-chains. Therefore, insertion of amphipathic helices causes curvature of these membranes. On the other hand, the inner leaflet of the plasma membrane is rich in negatively charged lipids (i.e. PS, PI4P, PI $\left.(4,5) \mathrm{P}_{2}\right)$ which makes these membranes strongly negatively charged. Two main families of curvature sensors are (i) BAR domain containing proteins that interact with the negatively charged membranes through their positive surface patches (Mim \& Unger, 2012) and (ii) ALPS motifs which are amphipathic helices that contain non-polar side chains on their hydrophobic surface used to screen lipid packing defects (Bigay \& Antonny, 2012). Proteins containing BAR and ALPS motifs further stabilize the curved membrane regions. It is important to note that many intracellular organelles also contain negatively charged lipids mostly from phosphoinositide family (Di Paolo \& De Camilli, 2006). These lipids are especially important in signaling and recruitment of specific cytosolic proteins that require protein and lipid signal for binding to the membrane, the so-called 'coincidence detection'. 

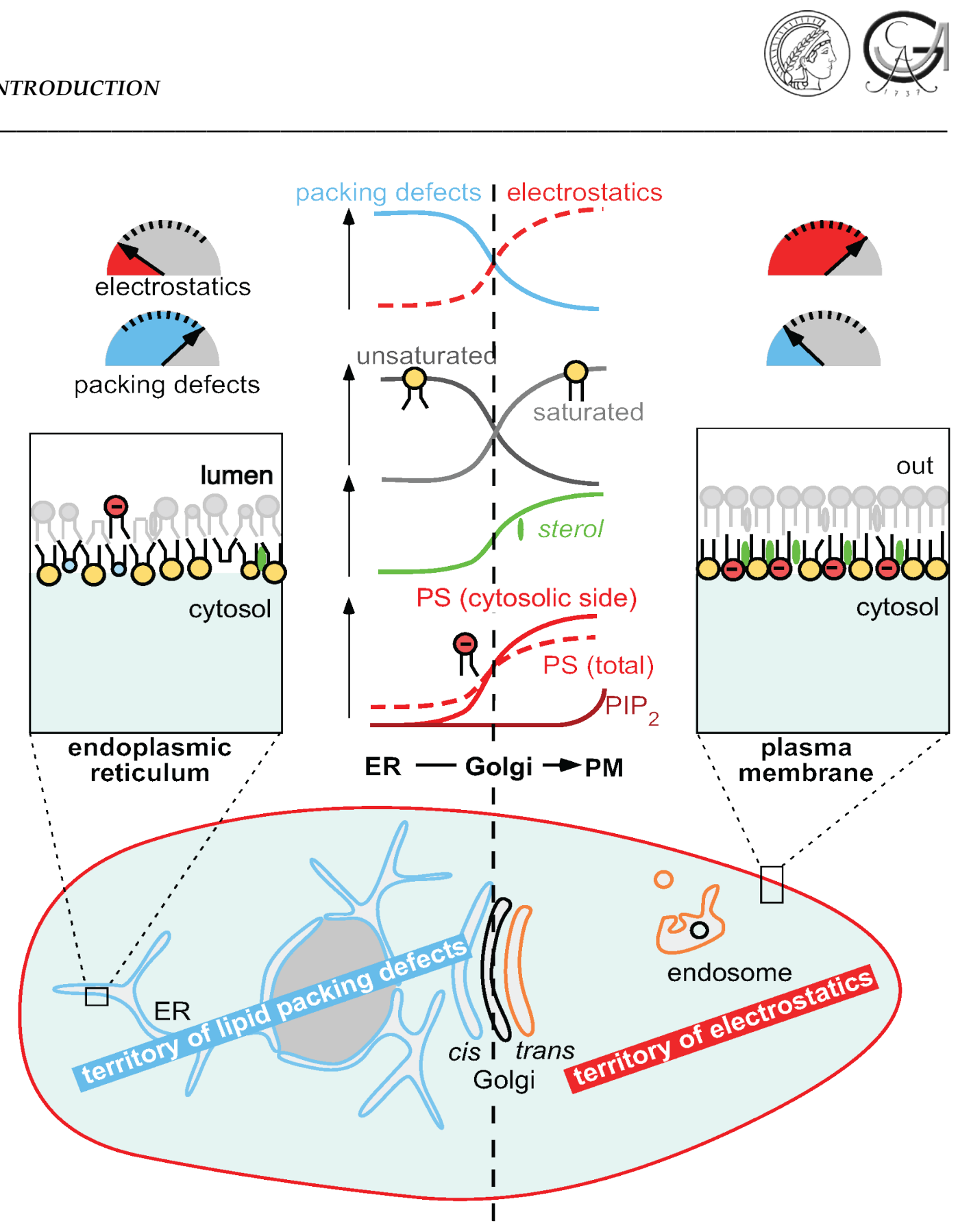

Figure 1.9. Different biochemical properties of lipids generate two major membrane territories in cell. First is the territory of lipid packing defects: ER and cis-Golgi contain more unsaturated lipids with less charged head-groups. Second is the territory of electrostatics: trans-Golgi and plasma membrane contain more saturated lipids which head-groups are charged. Adapted from Bigay \& Antonny, 2012. 


\subsubsection{Lipid asymmetry in cellular membranes}

Apart from rotational and lateral diffusion, lipids in cellular membranes are distinctly distributed between two leaflets (Fig. 1.10A). In the plasma membrane of eukaryotic cells, PC and SM are present predominantly in the outer leaflet. On contrary, PE, different PIPs and PS are concentrated in the inner leaflet (van Meer, Voelker, \& Feigenson, 2008). The transfer of different lipid species between two leaflets is called flip-flop diffusion. In protein-free bilayers, spontaneous flip-flop diffusion leads to equilibration of lipid components (Gurtovenko \& Vattulainen, 2007).

In cellular membranes phospholipid scramblases do not require energy and stimulate bi-directional movements of lipids thereby counteracting the asymmetry (Fig. 1.10B). In contrast, two classes of integral membrane proteins maintain the bilayer asymmetry. First are ATP-binding cassette (ABC) transporters that use ATP to move lipids from the inner, cytosolic to the outer leaflet. Second are aminophospholipid translocases (APLTs) that selectively pump PS and PE from the outer to the inner leaflet maintain the bilayer asymmetry (Daleke, 2008).

Segregation of lipid biosynthesis/turn-over enzymes at different membranes contributes as well to their asymmetric distribution (i.e. glycolipid synthesis in the outer leaflet and $\mathrm{PI}(4,5) \mathrm{P}_{2}$ turn-over in the inner leaflet of the plasma membrane). Hence, PIs are localized at the membrane/cytosol interface where can be recognized by a variety of cytosolic proteins through PI-interacting domains. Also, PIs have a unique distribution throughout the cell and large number of PI-kinases and PI-phosphatases are necessary to maintain organelle identity in respect to distinct PI species (Di Paolo \& De Camilli, 2006). 


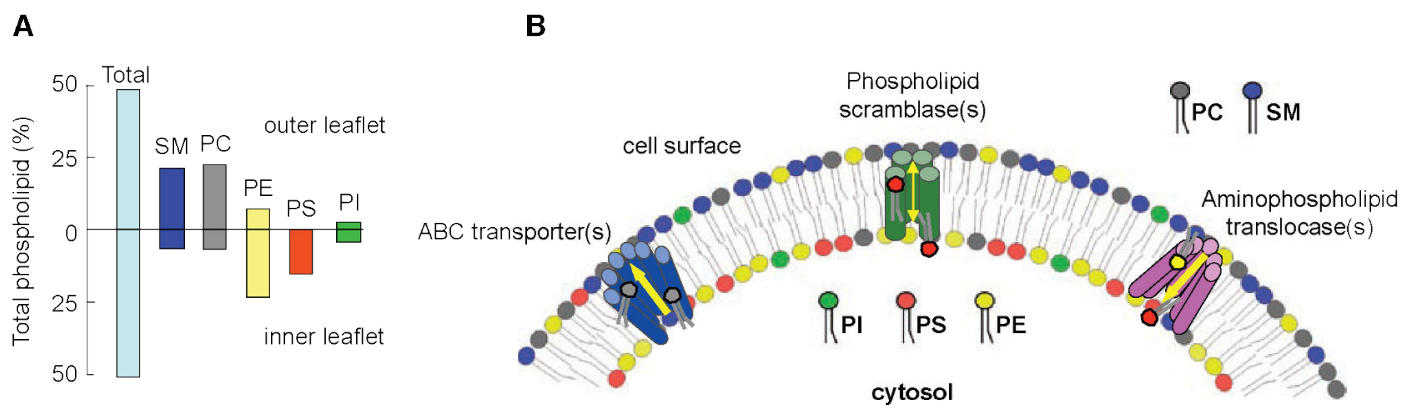

Figure 1.10. Lipid asymmetry in the plasma membrane. A. Relative abundance of different phospholipids: PC and SM are mostly distributed in the outer leaflet, PE, PS and PI are mostly in the inner leaflet. B. The asymmetry of the plasma membrane is mediated by (i) phospholipid scramblases, (ii) ATP-binding cassette (ABC) transporters and (iii) aminophospholipid translocases. Adapted from (Fadeel \& Xue, 2009; Daleke, 2008).

\subsubsection{Phase separation in cellular membranes}

The first idea that biological membranes contain adjacent phases under physiological conditions came from the difference in solubility of certain lipid species during detergent extraction (Simons \& van Meer, 1988). Certain proteins preferentially accumulated in these 'detergent-resistant' lipid patches during extraction with non-ionic detergents (Brown \& Rose, 1992; Schroeder, London, \& Brown, 1994). It was then proposed that these complexes represent specific lipid domains in the plasma membrane, so-called lipid rafts, to which certain proteins preferentially associate (Fig. 1.11A; Simons \& Ikonen, 1997).

Further characterization of protein separation in reconstituted membranes showed that most of the proteins partition into the L $\alpha$ phase and that a minor subset of proteins accumulate in the Lo-phase/rafts (R. F. M. de Almeida, Fedorov, \& Prieto, 2003; R. de Almeida et al., 2004). However, such a detergent treatment has inherited problems: (i) extraction at low temperature induces lipid phase transition, (ii) detergents severely disrupt the membrane structure since it solubilizes particular lipids rupturing the membrane, (iii) dehydration of the membrane interface ruins the hydrogen bonding at the surface (i.e. electrostatic repulsion between polyionic patches of proteins and/or lipid head-groups becomes stronger) which does not affect 
all domains at the same extent (R. F. M. de Almeida et al., 2003; Heerklotz \& Seelig, 2002; Jesús Sot, M Isabel Collado, José L R Arrondo, Alicia Alonso, \& Goñi, 2002; E. London \& Brown, 2000; Silvius, del Giudice, \& Lafleur, 1996). Therefore, proteins that accumulate in these detergent resistant regions should be seen as potential candidates associating in domains, rather than the proof of such association. Some proteins such as GPI-anchored thymocyte antigen 1, ganglioside GM-1 and the membrane spanning linker for activation of $\mathrm{T}$ cells are all enriched when purified using DRMs, but all of these proteins are shown to form distinct clusters in the plasma membrane (Lichtenberg, Goñi, \& Heerklotz, 2005; Wilson et al., 2004).

Nonetheless, the concept of specific protein/lipid domains in the membrane was instrumental in establishing the significance of the lateral organization in the cellular context. Moreover, increasing evidence indeed show that particular lipids would segregate around certain domains in order to best accommodate ("lubricate") the integral proteins which thicknesses can vary greatly (Fig. 1.11B; Anderson \& Jacobson, 2002).

Recent assays showed phase transitions in vesicles formed from blebs of the plasma membrane, so-called giant plasma membrane derived vesicles (GPMVs) supporting the idea that lipid phase partitioning is a major factor in generating subdomains within the membrane plane (Levental, Grzybek, \& Simons, 2011; Sezgin et al., 2012). However, in GPMVs we have a loss in lipid asymmetry between the leaflets. Also, cytoskeleton elements are detached from the membrane allowing for large-scale lipid mixing (Charras, Yarrow, Horton, Mahadevan, \& Mitchison, 2005). Rafts are currently envisioned to be much smaller in size (few dozen $\mathrm{nm}$ ) with faster protein and lipid exchange (Lingwood \& Simons, 2010; Pike, 2006). Using stimulated emission depletion (STED) nanoscopy in combination with fluorescence correlation spectroscopy (for more details look at Materials and Methods), GPI-anchored proteins were observed to be trapped in domains of $\sim 20 \mathrm{~nm}$ for around $10 \mathrm{~ms}$ (Eggeling et al., 2009). Moreover, owing to the development of superresolution optical microscopy, it is becoming clear that solely a phase-transition model cannot explain that many nanometer-sized clusters alone but that multiple different mechanisms shape the protein and lipid lateral distribution in the plasma membrane (Saka et al., 2014; Sieber et al., 2007; Wilhelm et al., 2014). 


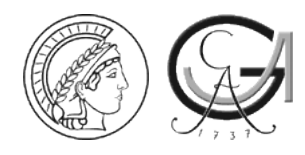

A
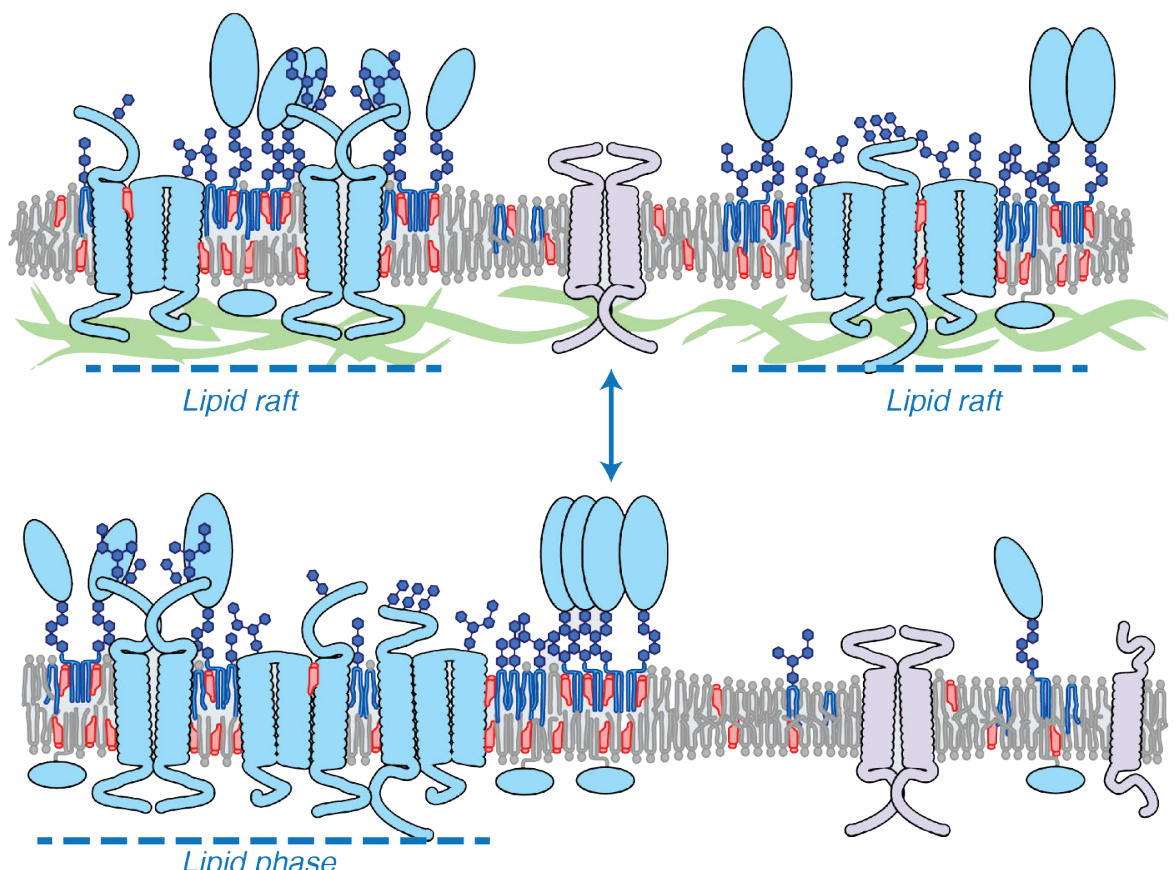

B

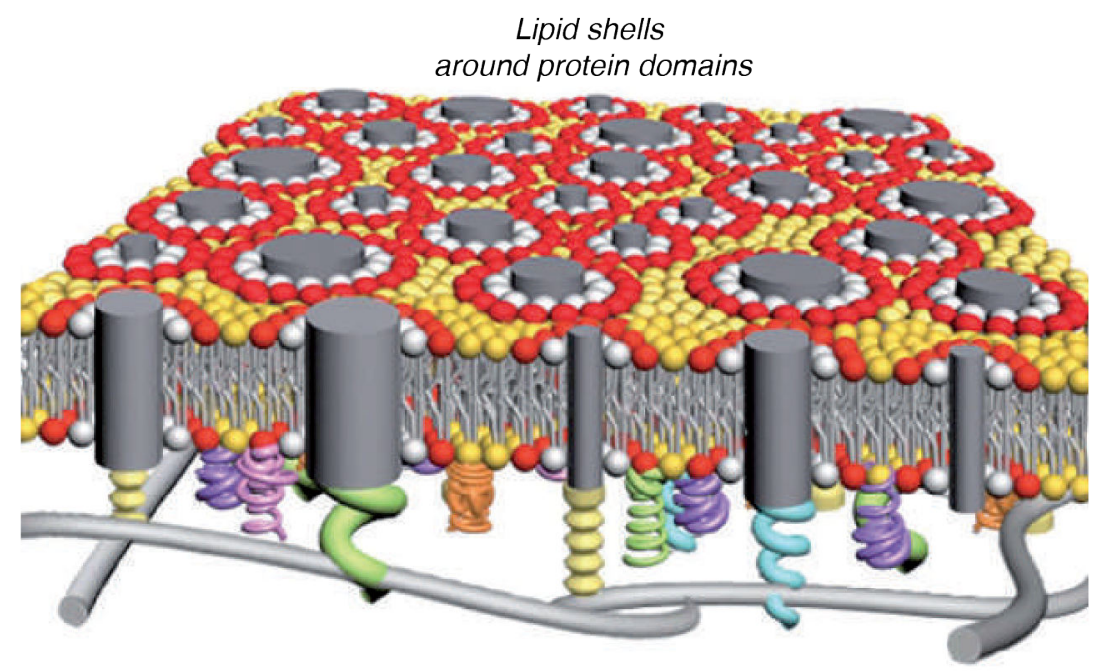

Figure 1.11. Lipid phases in the plasma membrane. A. Lipid rafts enriched in cholesterol and SM sequester GPI-anchored proteins and certain TMDs. These nanoscale rafts coalesce into larger raft phases that may resemble the Lo phases in reconstituted membranes. B. TMDs of particular thickness will sequester lipids with that would best accommodate these TMDs, thereby generating nanoscale domains. Adapted from Lingwood \& Simons, 2010 and Anderson \& Jacobson, 2002. 
1.2.5 Cytoskeleton and scaffolding proteins fine-tune the plasma membrane structure

Single particle tracking and fluorescence correlation spectroscopy experiments suggested that the formation of any large protein-lipid phase is prevented in the plasma membranes (D. M. Owen, Williamson, Rentero, \& Gaus, 2009). This may partially be due to an underlying actin meshwork that acts as a fence together with some of the membrane proteins docked into this meshwork as pickets, the so-called picket-fence model (Fig. 1.12A; Dietrich, Yang, Fujiwara, Kusumi, \& Jacobson, 2002; Fujiwara, Ritchie, Murakoshi, Jacobson, \& Kusumi, 2002; Jacobson, Hou, Derzko, Wojcieszyn, \& Organisciak, 1981). Indeed, in electron microscopy images of the plasma membrane it is clear that cytosolic meshwork generates the membrane regions of maximally $300-400 \mathrm{~nm}$ in size (Fig. 1.12B; Morone et al., 2006).

The plasma membrane of most eukaryotic cells is underlined with the spectrin-based protein network that affects cell shape and elastic properties. Spectrin is a flexible, rod-shaper antiparallel heterotetramer composed of $\alpha$ and $\beta$ - spectrin (Bennett \& Baines, 2001). Spectrin connects filamentous actin (F-actin) with the proteins that structurally support the plasma membrane/interact with integral proteins (e.g. ankyrin, protein 4.1, adducing, catenin). F-actin fibers organize the cortical actin that both provides the cell stability as well as allows the formation of tissue by coupling to the extracellular matrix (Clark \& Brugge, 1995; Pantaloni, Le Clainche, \& Carlier, 2001). For instance, on the cytosolic side F-actin interacts with the cytosolic adaptor proteins (such as vinculin, talin, a-actinin) that associate with the integrins; on the extracellular side integrins attach to the extracellular matrix molecules. These adaptor proteins also directly bind to the inner leaflet lipid $\mathrm{PI}(4,5) \mathrm{P}_{2}$ (Gilmore \& Burridge, 1996).

Another way of F-actin interaction with the plasma membrane is through ERM family of proteins (ezrin, radixin and merlin) (Bretscher, Edwards, \& Fehon, 2002). These proteins attach to the membrane through their N-terminal and crosslink F-actin via the C-terminal. Lipids such as $\mathrm{PI}(4,5) \mathrm{P}_{2}$ induce the transient conformational opening of the ERM molecules (separation of $\mathrm{N}$ - and C-terminals) and ERMs are further stabilized in the open conformation by phosphorylation at their C-terminal (Fehon, McClatchey, \& Bretscher, 2010). 


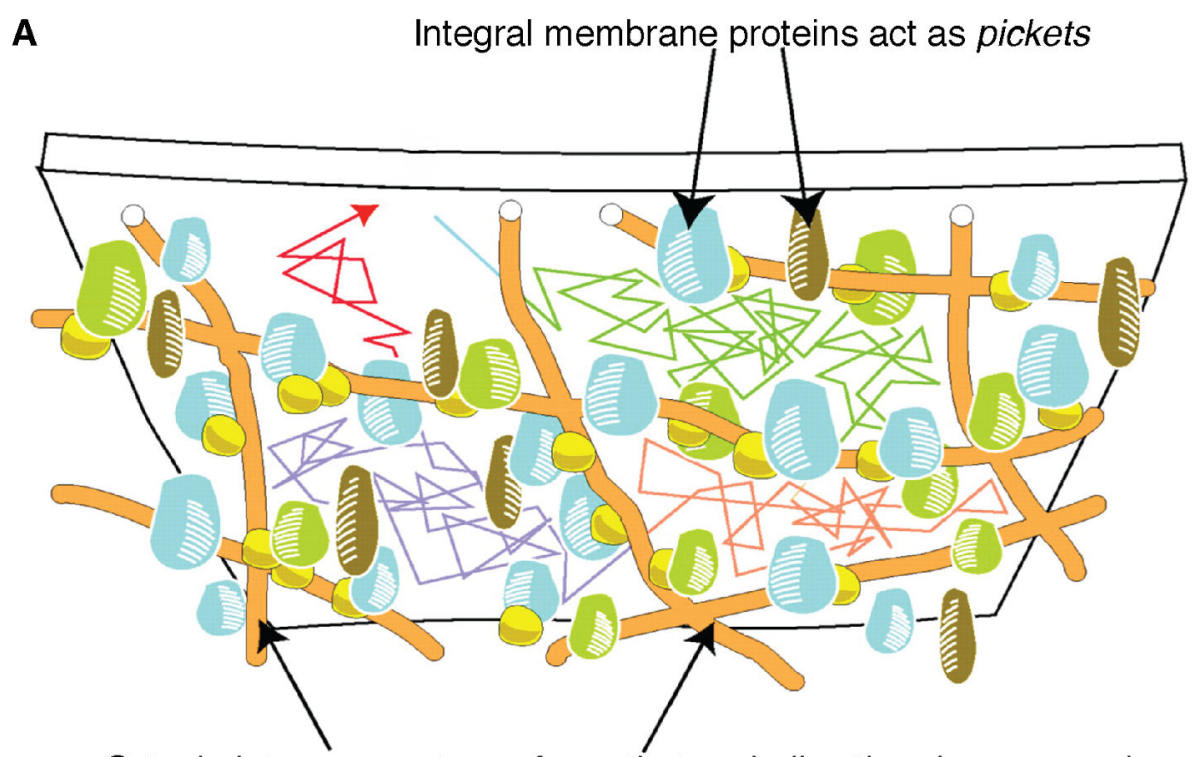

Cytoskeleton generates a fence that underlies the plasma membrane
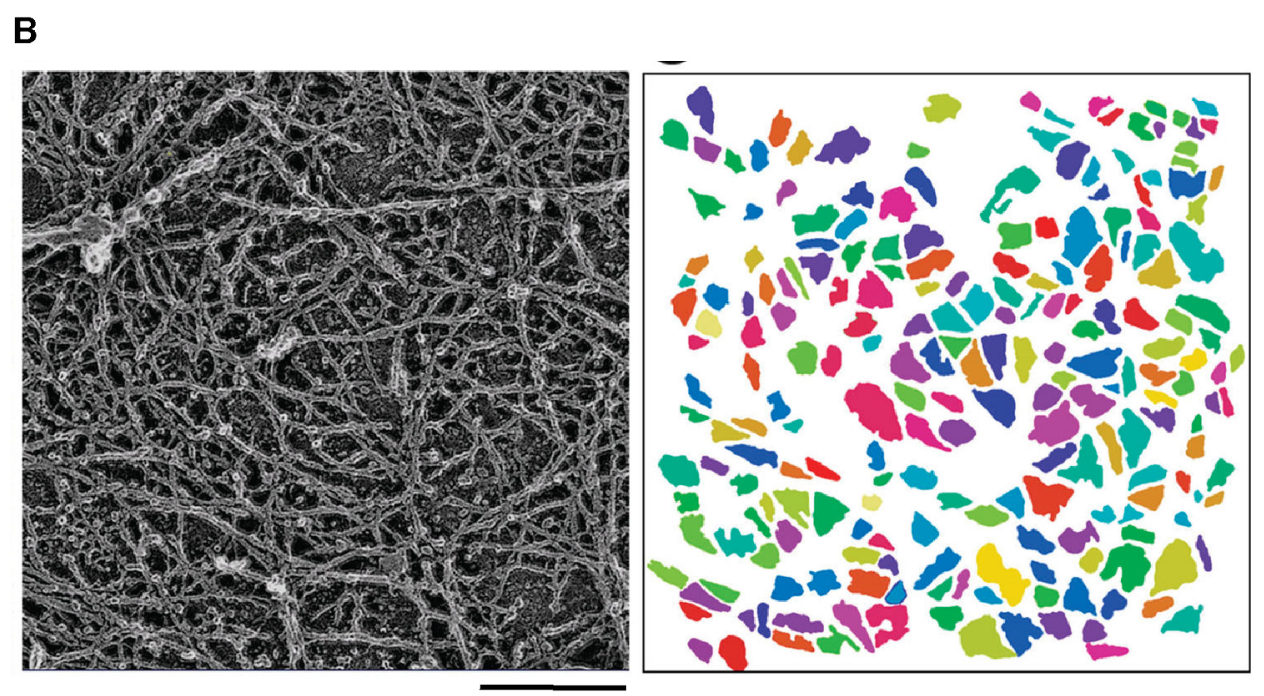

Figure 1.12. Picket-fence model of the plasma membrane organization A. Scheme of the cortical actin underlying the plasma membrane. Certain proteins in the plasma membrane (pickets) interact to the actin network directly or through adaptor proteins. Actin network prevents coalescence of smaller domains into larger phases. B. EM images (inside-out) of the actin network that lines up the inner leaflet of the plasma membrane. Scale bar 200 nm. Adapted from Kusumi, Suzuki, Kasai, Ritchie, \& Fujiwara, 2011 and Morone et al., 2006. 
Spectin and actin networks are just the examples how cytosolic scaffold proteins can alter the membrane structure. Some membranes such as pre- and postsynaptic neuronal membranes depend less on the actin meshwork. In the presynapse, specific scaffolding proteins (such as Rab3-interacting molecule (RIM), piccolo, bassoon and ELKS/Rab6-interacting/CAST family (ERC), MINT1, liprin $\alpha$ ) define the region of synaptic vesicle tethering, docking and subsequent release sites (Ziv and Garner, 2004; Fernández-Busnadiego et al., 2013; 2010; Imig et al., 2014). For instance, in mammalian synapses RIM interacts with the synaptic vesicle proteins Rab3 and synaptotagmin, as well as with calcium channels in the plasma membrane. This mechanism thus results in a indirect association of the synaptic vesicle to the calcium channel (Fernández-Busnadiego et al., 2013; Rosenmund et al., 2002) and ensures a tight spatial coupling between calcium influx and NT release (Fig. 1.13). Further molecular details of the precise organization of the presynaptic active zone are still emerging. In parallel, the organization of receptors in the postsynapse depends again on specific protein scaffolds such as PSD 95 in excitatory (Cline, 2005; de Bartolomeis \& Tomasetti, 2012) and gephyrin in some inhibitory postsynapses (Papadopoulos \& Soykan, 2011).

The presynapse and postsynapse are not isolated entities, but rather actin rearrangements at the postsynapse can influence the organization and release efficiency in the presynapse (Cheadle \& Biederer, 2012; Dean \& Dresbach, 2006; Ziv and Garner, 2004). Synaptic formation can be triggered by the presynaptic (axonal) membrane, postsynaptic (dendritic) membrane or by both membranes simultaneously contacting each other. Postsynaptic adhesion molecules have been shown to play a key role in synapse maturation (ElHusseini, Schnell, Chetkovich, Nicoll, \& Bredt, 2000; Wittenmayer et al., 2009) and activity (Regalado, Terry-Lorenzo, Waites, Garner, \& Malenka, 2006; Woolfrey et al., 2009). Adhesion molecules thus modulate signaling across the synaptic cleft (Futai et al., 2007) thereby acting as both structural and information bridges between the postsynapse and presynapse. Thus the cytoskeleton and scaffolding proteins in the cytosol modulate the plasma membrane structure and dynamics, and this not only holds for neurons but for many cell types. 


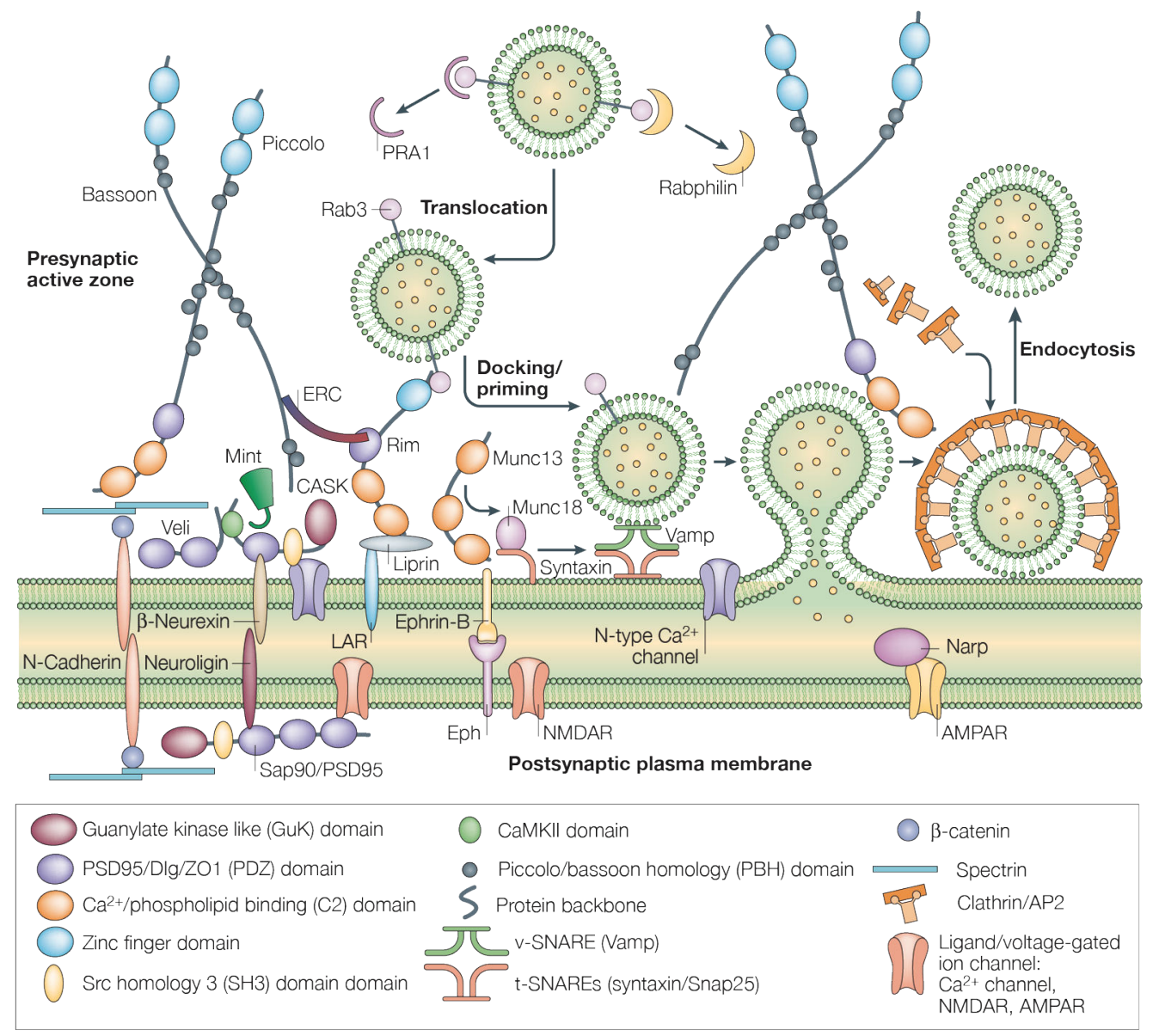

Figure 1.13. Organization and coupling of the excitatory pre- and postsynapse. Presynaptic scaffolding proteins organize synaptic vesicles and $\mathrm{Ca}^{2+}$-channels. Presynapse and postsynapse are coupled through the interactions of $\beta$-neurexins/neuroligins, ephrinB/Eph and $\mathrm{N}$-cadherins. Adapted from Ziv and Garner, 2004. 


\subsection{SNARES AS TOOLS TO UNDERSTAND THE PHYSICAL PRINICIPLES BEHIND MEMBRANE PATTERNING}

\section{Section 1.3 was published in:}

Milovanovic, D. \& Jahn, R. (2015). Frontiers in Physiology 6:89 http:// doi: 10.3389/fphys.2015.00089

Proteins involved in synaptic vesicle release have served as excellent models for analyzing the patterning of the plasma membrane. Synaptic vesicle release itself is a well-orchestrated process where a neurotransmitterloaded vesicle attaches to the plasma membrane (a process known as docking), after which the fusion machinery enters a 'preparatory' phase (known as priming) and then, once there is a calcium influx, the vesicle fuses with the plasma membrane (Südhof, 2004). Membrane fusion, the key step in neurotransmitter release, is mediated by the interaction between protein members of the soluble NSF-attached protein receptor (SNARE) family that reside in the donor membrane with their cognate partners in the target membrane (Hong \& Lev, 2014; Jahn \& Scheller, 2006).

SNARE proteins posses a central 60-70 AA-long motif (SNARE domain) that forms a coiled coil upon the interaction with the cognate SNARE partners. This coiled coil is connected by sixteen layers of interacting amino acid side chains that are hydrophobic (the flanking are all polar or charged) except of the amino acids in the central layer, which are either glutamine (Q) or arginine (R). Generally, the coiled-coil SNARE complex has three domains that contain glutamine together with one that contains arginine (QabcR) (Antonin, Fasshauer, Becker, Jahn, \& Schneider, 2002; Stein, Weber, Wahl, \& Jahn, 2009; Sutton, Fasshauer, Jahn, \& Brunger, 1998). The SNAREs involved in neuronal exocytosis include the plasma membrane residents syntaxin $1 \mathrm{~A}$ (Qa) and SNAP 25 that contributes with two SNARE motifs (Qbc), and synaptobrevin 2 at the synaptic vesicle (R). Other SNARE complexes mediate intracellular traffic steps such as early and late endosomal fusion (Fig. 1.14). 

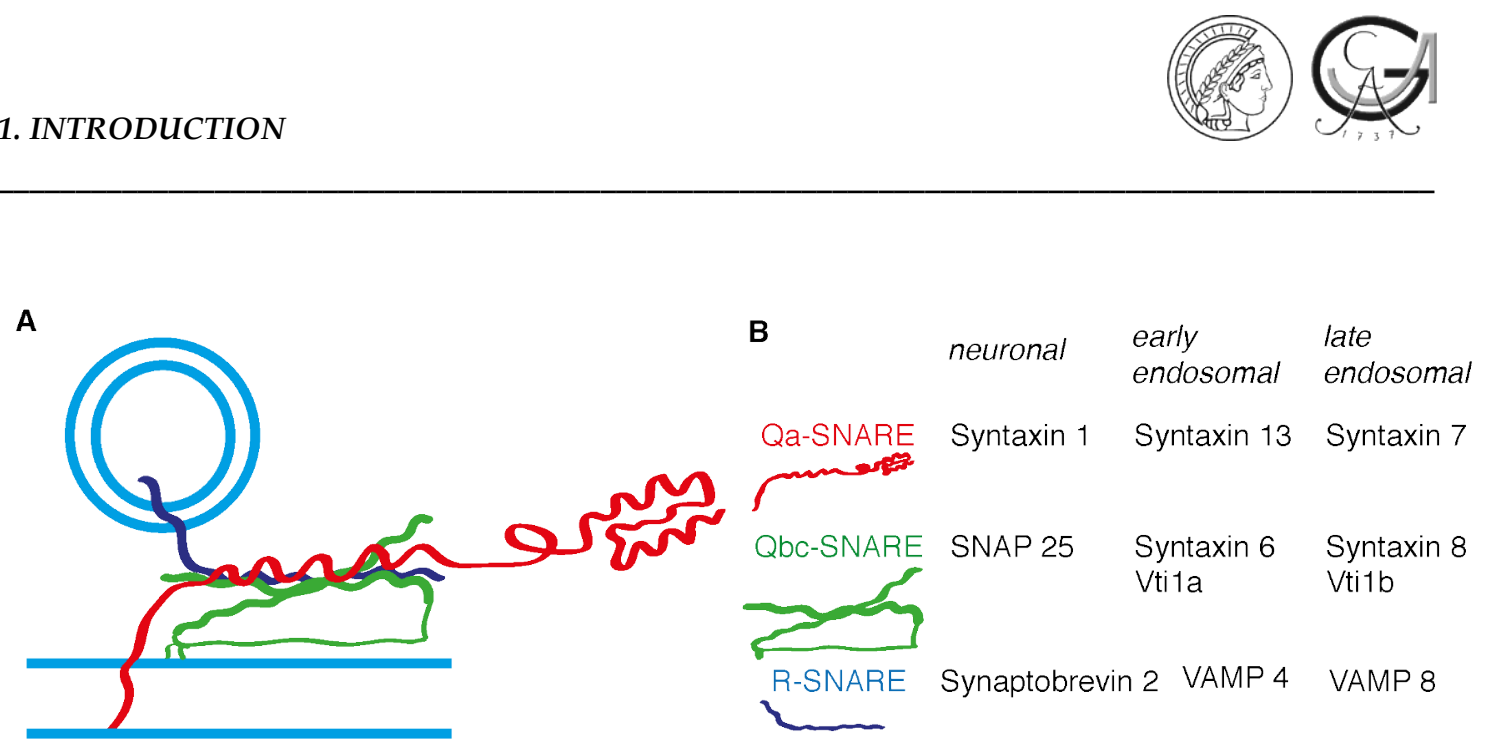

Figure 1.14. SNARE proteins catalyze membrane fusion. A. Scheme of the vesicle (with R-SNARE) approaching the plasma membrane (with Qabc SNAREs). Helical motifs form QabcR SNAREs zipper in N- to C-terminal direction forming the four-helical bundle. B. Examples of some cognate SNARE proteins involved in neuronal, early and late endosomal fusion.

In recent years, an increasing body of evidence has revealed that SNAREs form clusters in both plasma membranes and intracellular membranes. Multiple approaches have yielded an increasingly refined picture of the forces and of the other biophysical parameters responsible for SNARE clustering, which will be discussed in the following chapters.

\subsubsection{SEGREGATION WITHIN THE HYDROPHOIBC CORE OF THE MEMBRANE}

Clustering induced by lipid phases. As discussed above, lipid-based domain segregation was first postulated based on the observation that certain proteins tend to associate with specific lipid species (most conspicuously with cholesterol and sphingomyelin) and resist extraction by some nonionic detergents. Although initial studies reported enrichment of SNAREs within DRMs (Chamberlain \& Gould, 2002; Chamberlain, Burgoyne, \& Gould, 2001; Lafont et al., 1999; S. A. Predescu, Predescu, Shimizu, Klein, \& Malik, 2005; Salaün, Gould, \& Chamberlain, 2005), it soon became clear that by applying different detergents, SNAREs were not co-floating with the classical DRM markers (Lang et al., 2001; Ohara-Imaizumi, Nishiwaki, Kikuta, et al., 2004a). 
However, similar to DRMs cholesterol is required for the integrity of SNARE clusters (Lang, 2007; Lang et al., 2001). Furthermore, cholesterol depletion inhibits exocytosis in both neuronal (Lang et al., 2001) and non-neuronal cells such as epithelial (Chintagari et al., 2006) and endothelial cells (S. A. Predescu et al., 2005), but it is still unclear whether dispersal of SNARE clusters and inhibition of fusion are causally related. Beyond neurotransmitter release, SNARE clusters are shown to be the release sites for cytokines at the phagocytic cup (Kay, Murray, Pagan, \& Stow, 2006) and insulin (OharaImaizumi, Nishiwaki, Kikuta, et al., 2004a; Ohara-Imaizumi, Nishiwaki, Nakamichi, et al., 2004b). Additionally, in vitro reconstitution of neuronal SNARE proteins into giant unilamellar liposomes capable of undergoing phase segregation suggested that SNAREs distribute in the liquid disordered phase (unsaturated phospholipids, cholesterol depleted regions), rather than in the liquid ordered phases (rich in saturated phospholipids and cholesterol). Although such simple phase-separation may not reflect phase partitioning in the plasma membranes, these studies confirmed that SNAREs do not associate with sphingomyelin and saturated phospholipids (Bacia, Schuette, Kahya, Jahn, \& Schwille, 2004; Saslowsky, Lawrence, Henderson, \& Edwardson, 2003). On the other hand they demonstrate that SNARE proteins are sensitive to such phase partitioning, raising the possibility that phase heterogeneity may contribute to SNARE segregation.

Clustering induced by hydrophobic mismatch. Hydrophobic mismatch occurs when the length of the protein transmembrane domains (TMDs) does not match the bilayer thickness (Fig. 1.15). In this case, it is energetically favorable to cluster the TMDs of similar length in the same region rather than to accommodate each of the TMDs separately. In a theoretical paper, Mouritsen and Bloom proposed that proteins may cluster in order to minimize membrane mismatch (Mouritsen \& Bloom, 1984). Pioneering research showed that certain enzymes have the highest activity when reconstituted in bilayers of particular thickness, whereas in both thinner and thicker bilayers the activity drops (Johannsson et al., 1981a; Johannsson, Smith, \& Metcalfe, 1981b; Kusumi \& Hyde, 1982). This implied that hydrophobic mismatch affects enzyme conformation that subsequently reduces its activity. Moreover, the aggregation state of some of these proteins 
such as rhodopsin is shown to depend on the acyl-chain length of lipids that were used for the reconstitution (Kusumi \& Hyde, 1982). More recently, it was shown that the overlap between the TMD length of the perfringolysin $\mathrm{O}$, a multispanning barrel protein, and the width of lipid bilayer also affects the proteins' distribution and functionality in proteoliposomes (Lin \& London, 2013). Protein clustering driven by hydrophobic mismatch was first shown directly for synthetic TMD peptides (de Planque et al., 1998; Sparr et al., 2005). The phospholipid acyl-chains are flexible and their lateral organization depends on the neighboring lipid molecules (i.e. cholesterol restricts the flexibility due to the pronounced hydrophobic planar structure). Hence, lipids can adapt to a range of different thicknesses (Killian \& Nyholm, 2006). On the other hand, proteins exhibit less flexibility in length distortion in the case of membrane mismatch (Petrache et al., 2002; Zaccai, 2000). Caution is needed when interpreting experiments based on altering acyl chain lengths because these changes also affect the lipid packing, curvature and surface charge distribution. Hence, the protein function may be affected by many of these parameters (Anderson \& Jacobson, 2002).

Hydrophobic mismatch appears to play a role in defining the final destination of membrane components in intracellular trafficking. It is well established that sorting of proteins and lipids in polarized, epithelial cells is mediated by both the lipid environment and the cytoskeleton, and that lipid domains coalesce prior to vesicle formation (Brown \& Rose, 1992; Lipowsky, 1993; Roux et al., 2005; Yoshimori, Keller, Roth, \& Simons, 1996). Additionally, altering the TMD length of peptides affected their trafficking from ER, Golgi to the plasma membrane. Considering that the average thickness of the membrane increases from ER $(\sim 3.75 \mathrm{~nm})$ to the plasma membrane ( $\sim 4.25 \mathrm{~nm}$; Mitra, Ubarretxena-Belandia, Taguchi, Warren, \& Engelman, 2004), it is reasonable to expect that proteins destined to the plasma membrane have longer TMDs. Indeed, when the TMD of plasma membrane syntaxin 3 was truncated to be 17 instead of endogenous 23 AA, syntaxin 3 was retained in the cis-Golgi (Watson \& Pessin, 2001). Also, in a comprehensive screen of the TMDs sequences from different species, Sharpe et al. demonstrate that an average length of TMDs is about 5 amino acids shorter for proteins destined to ER compared to the proteins of the plasma membrane (Sharpe, Stevens, \& Munro, 2010). 
A

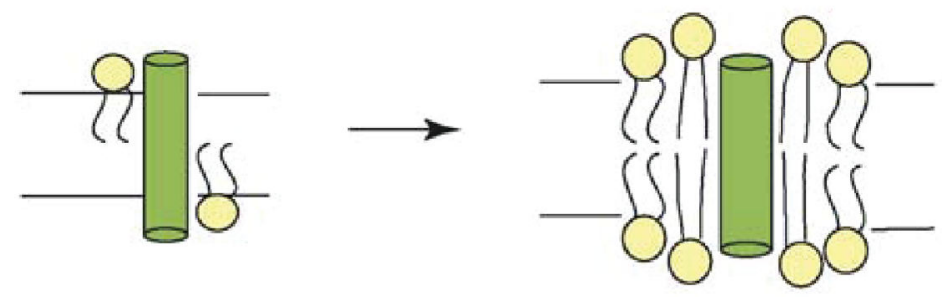

B

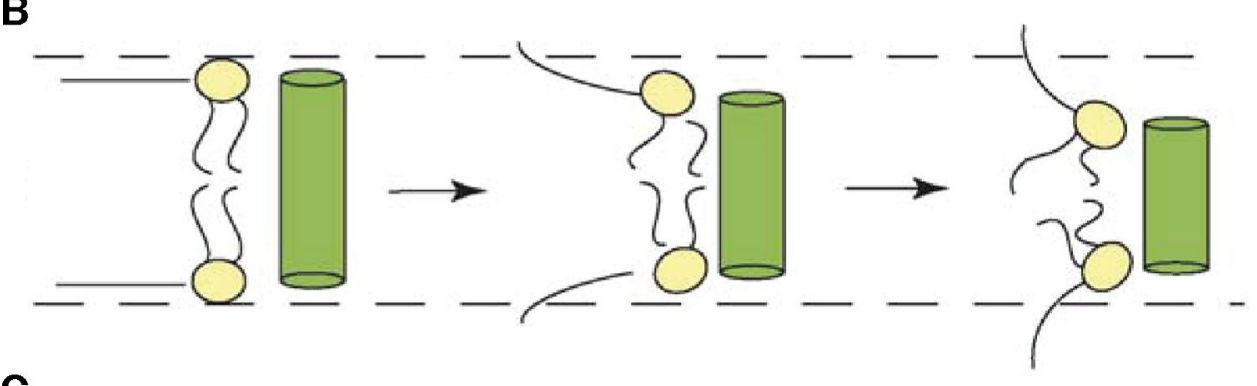

C

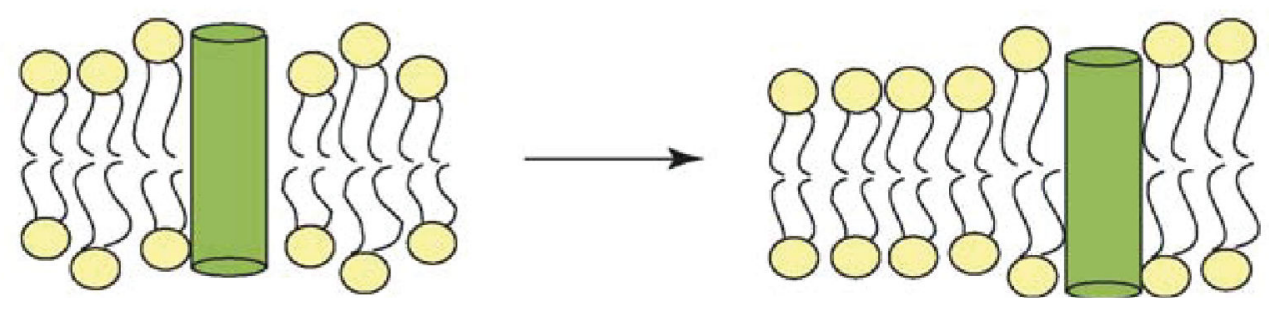

Figure 1.15. Effects of hydrophobic mismatch between lipids and proteins. A. Lipids will stretch acyl chains next to the longer TMD (positive mismatch). B. Disordering of the acyl chains of lipids next to the shorter TMD (negative mismatch). C. In the complex mixture of lipids, TMD sequesters the lipids that best match its thickness. Adapted from Killian \& Nyholm, 2006.

Palmitoylation modulates attachment of soluble proteins to the membrane. Posttranslational modifications further modulate SNARE patterning (for detailed review see (van den Bogaart, Lang, \& Jahn, 2013)). Most attention has been paid to palmitoylation, i.e. the covalent addition of the acyl chain palmitate (C16:0) to a cysteine residue in the protein. For instance, the Qbc SNAREs SNAP 23 and 25 are palmitoylated at five and four cysteine residues, respectively, which is required for membrane attachment (Prescott, Gorleku, Greaves, \& Chamberlain, 2009). Proteomics analyses suggested that many other synaptic proteins undergo palmitoylation including proteins containing TMDs (Kang et al., 2008), among these are the SNAREs syntaxin 1 and 
synaptobrevin 2. It has been suggested that SNAREs are reversibly targeted to cholesterol and sphingomyelin rich regions via palmitoylation (Y. Fukata \& Fukata, 2010; Levental, Lingwood, Grzybek, Coskun, \& Simons, 2010), which would add another mechanism contributing to cluster formation. Support for this concept is provided by the recent finding that a fraction of amyloid precursor is palmitoylated, which further modulates its association with cholesterol-rich regions in the presynaptic membrane (Bhattacharyya, Barren, \& Kovacs, 2013).

\subsubsection{SEGREGATION CAUSED BY INTERACTIONS AT THE HYDROPHOBIC-HYDROPHILIC BOUNDARY}

Clustering of SNAREs is influenced by electrostatic interactions between positively charged side chains adjacent to the hydrophobic TMD with negatively charged polyphosphoinositides (Di Paolo \& De Camilli, 2006; Do Heo et al., 2006; van den Bogaart et al., 2011). Tamm and colleagues showed that diffusion of syntaxin 1 decreases upon incorporation of $\mathrm{PI}(4,5) \mathrm{P}_{2}$ in the lipid monolayer (Wagner \& Tamm, 2001). $\mathrm{PI}(4,5) \mathrm{P}_{2}$ is enriched in regions of the plasma membrane where secretory vesicles dock (Aoyagi et al., 2005; Laux et al., 2000), and it is essential for exocytosis (Hay \& Martin, 1993; James et al., 2008; Milosevic et al., 2005; Wen, Osborne, \& Meunier, 2011). Although $\mathrm{PI}(4,5) \mathrm{P}_{2}$ comprises only $1 \%$ of total lipids of the plasma membrane (Di Paolo \& De Camilli, 2006), it can reach concentrations of more than $80 \%$ of total lipids in clusters (van den Bogaart et al., 2011). Association between syntaxin 1 and $\mathrm{PI}(4,5) \mathrm{P}_{2}$ was clearly shown both in vitro reconstituted systems (Murray \& Tamm, 2009; 2011) and in cells (van den Bogaart et al., 2011). Responsible for this strong interaction is a cluster of positively charged arginines and lysines directly adjacent to the TMD of syntaxin 1 (Fig. 1.16; (Khuong et al., 2013; van den Bogaart et al., 2011)). 
A

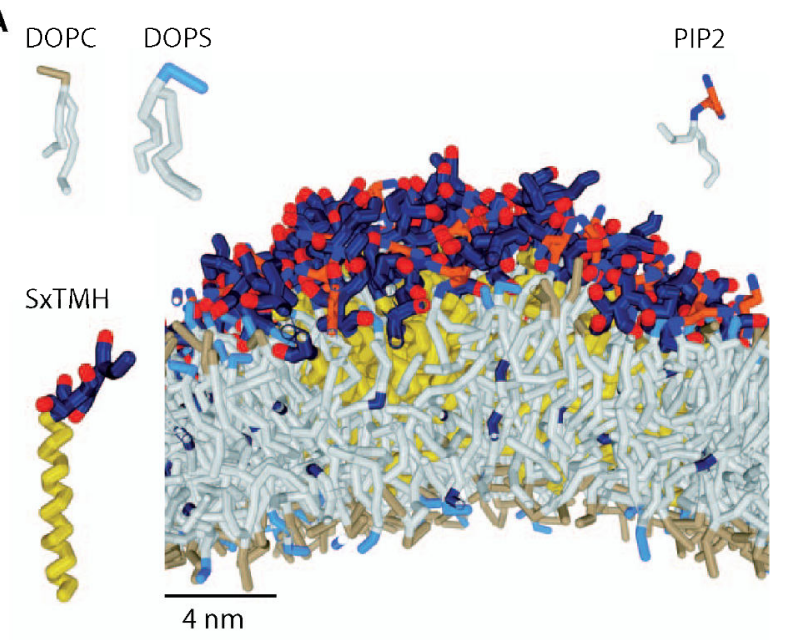

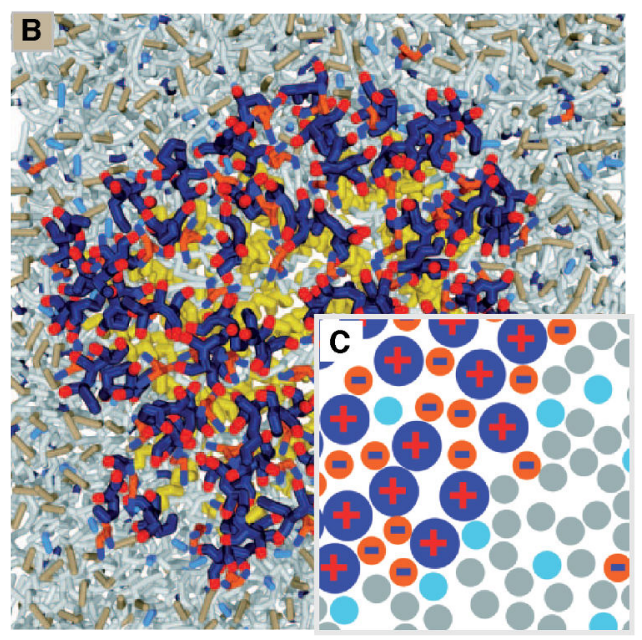

Figure 1.16. Syntaxin $1 \mathrm{~A}$ interacts with $\mathrm{PI}(4,5) \mathrm{P}_{2}$ in the membrane. Side view (A), top view (B) and scheme (C) of a coarse-grained molecular dynamics simulation (64 copies of syntaxin 1A peptide incorporated in the DOPC:DOPS mixture (4:1 molar ration); simulation time $6 \mu \mathrm{s})$. $\mathrm{PI}(4,5) \mathrm{P}_{2}$ interacts with the polybasic patch of syntaxin 1A that is juxtaposed to its TMD. Adapted from van den Bogaart et al., 2011.

Ionic interactions between macromolecules are strongly influenced by mobile ions. The ionic composition at the surface of a membrane is highly complex (Y.-H. Wang et al., 2012; Y.-H. Wang, Slochower, \& Janmey, 2014), rendering it difficult to quantify the influence of ions on domain formation. Ions present at high concentrations on the cytoplasmic surface $\left(\mathrm{K}^{+}, \mathrm{Mg}^{2+}\right.$, glutamate, ATP; (Beis \& Newsholme, 1975; Hess, Metzger, \& Weingart, 1982; R. E. London, 1991)) are able to shield the charge of both lipid head-groups and proteins involved in exocytosis (Park et al., 2012). It is worth noting that calcium increases SNAREs clustering in the plasma membrane of PC12 cells (Zilly et al., 2011), and this mechanism might involve the interaction with negatively charged lipids. Polybasic clusters on the cytoplasmic face adjacent to transmembrane proteins are common among many membrane proteins (Heijne, 2006). Thus it is possible that such ionic interactions play a major role in patterning of the plasma membrane and possibly also of intracellular membranes. 
Both homophilic and heterophilic interactions have been described for Qa SNARE family members. For instance, syntaxin 1 and syntaxin 4 are involved in regulated and constitutive exocytosis, respectively. Interactions between the SNARE motifs at the cytoplasmic surface has been suggested to contribute to the segregation of these proteins into distinct domains (Fig. 1.17; (Sieber, Willig, Heintzmann, Hell, \& Lang, 2006)). Hence, in case of syntaxin isoforms homotypic protein interactions contribute to the functional segregation. Similarly, in non-neuronal cells, syntaxin isoforms segregate in different regions of the membrane. In highly polarized epithelial cells, syntaxin 3 and syntaxin 4 are trafficked distinctly to the apical and basolateral membrane, respectively. Even the deletion of the targeting signal of syntaxin 3 does not eliminate its distinct segregation from syntaxin 4 enriched regions (Low et al., 2006).

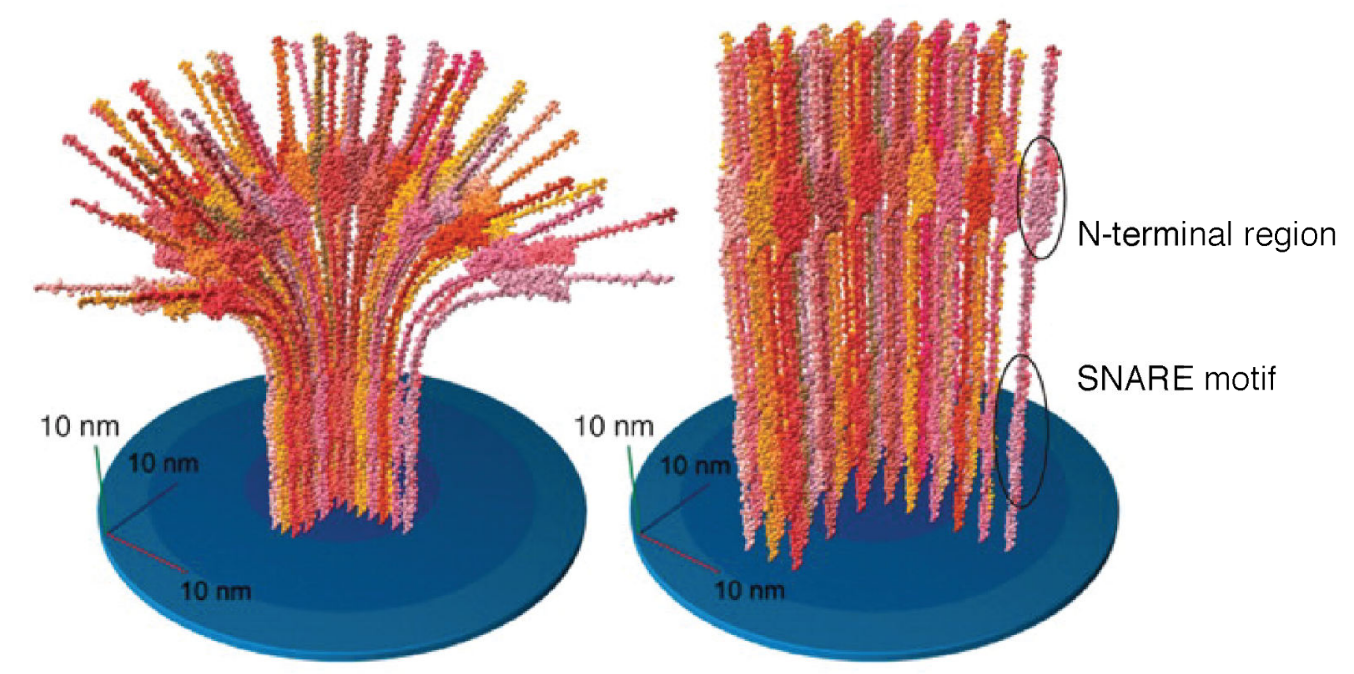

Figure 1.17. Supramolecular syntaxin clusters reconstituted in silico. Two possibilities exist for the shape of clusters: bunchlike (left) and cylindrical (right). The bunchlike organization is more feasible, since the overexpression does not change the size of cluster, but increases their number. Adapted from Sieber et al., 2007. 
Heterotypic protein interactions are important for both the spatial sorting of proteins in the presynapse, as well as for catalyzing the fusion reaction. For instance, some presynaptic membrane proteins bind to the actin meshwork (for details please look at Introduction 1.2.5; (Torregrosa-Hetland et al., 2013; 2011; Villanueva et al., 2012)). This binding to the cytoskeleton can be direct as in the case of syntaxin 4 (Jewell, Luo, Oh, Wang, \& Thurmond, 2008; Woronowicz et al., 2010) and SNAP 25 (Torregrosa-Hetland et al., 2013). Alternatively, binding to actin can be mediated by adaptor proteins such as myosin $\mathrm{V}$ that connects syntaxin 1 to actin (M. Watanabe et al., 2005), and $\alpha$ fodrin that connects syntaxins 3 and 4 to actin (Nakano, Nogami, Sato, Terano, \& Shirataki, 2001). Another example for heterotypic interactions includes binding of regulatory proteins to SNAREs. The SM-protein Munc 18 that binds to syntaxin 1 is not only essential for exocytosis (Verhage et al., 2000) but also necessary for trafficking of syntaxin 1 to the plasma membrane (Kurps \& de Wit, 2012; Voets et al., 2001; X. Yang, Xu, Xiao, Xiong, \& Xu, 2006). Indeed, if syntaxin 1 clusters serve as reservoir of the protein for fusion, Munc 18 may be needed to pry an individual syntaxin 1 molecules away from the cluster (Bar-On et al., 2012). Munc 18 bound syntaxin 1 is able to recruit SNAP 25 in the cell lawns and synaptobrevin 2 containing vesicles can bind to this complex (Zilly, Sørensen, Jahn, \& Lang, 2006).

\subsection{FUNCTIONAL RELEVANCE OF SNARE CLUSTERING}

\section{Section 1.4 was published in:}

Milovanovic, D. \& Jahn, R. (2015). Frontiers in Physiology 6:89 http:// doi: 10.3389/fphys.2015.00089

SNAREs clustering may be important for exocytosis. First, the high local concentrations of SNAREs at the plasma membrane may provide the functional pools of proteins necessary for the formation of SNARE complexes (Fig. 1.18). Clustering of SNAREs may also prevent nonproductive sidereactions of the highly reactive SANREs such as the formation of so-called "dead-end" complexes between syntaxin 1 and SNAP 25 incapable of fusion 
(Fasshauer \& Margittai, 2004). It has been shown that the plasma membrane of chromaffin cells lacks these dead-end complexes (Halemani, Bethani, Rizzoli, \& Lang, 2010). Along the same line, removal of cholesterol does not only affects the clustering of SNAREs, but also reduces the number of functionally active syntaxin 1/SNAP 25 complexes ready for ternary complex formation with synaptobrevin 2 (Rickman et al., 2010). Second, Q-SNARE domains (together with $\left.\mathrm{PI}(4,5) \mathrm{P}_{2}\right)$ may represent docking platforms for vesicles (de Wit et al., 2009; Imig et al., 2014; James et al., 2008). PI(4,5) $\mathrm{P}_{2}$ was shown to be enriched at the sites of vesicle fusion, and altering the amount of $\mathrm{PI}(4,5) \mathrm{P}_{2}$ affects the release capacities (de Wit et al., 2009; Milosevic et al., 2005). Therefore, $\mathrm{PI}(4,5) \mathrm{P}_{2}$ domains have been proposed to act as molecular beacons for vesicle recruitment to the membrane. Indeed, synaptotagmin 1 , the main calcium sensor at the synaptic vesicle, binds to syntaxin $1 / \mathrm{PI}(4,5) \mathrm{P}_{2}$ domains in the plasma domains (Honigmann et al., 2013).

Finally, clustering may help overcoming the energy barrier that needs to be overcome for membrane fusion in two ways. First, clustering alters the line tension around clusters in the plasma membrane which generates regions in the membrane more susceptible for membrane fusion and budding (Boucrot et al., 2012; Kozlov et al., 2014; Risselada, Bubnis, \& Grubmüller, 2014). Hence, the total number of SNARE complexes needed fur fusion is reduced (Hernandez, Kreutzberger, Kiessling, Tamm, \& Jahn, 2014; Mohrmann, de Wit, Verhage, Neher, \& Sørensen, 2010; van den Bogaart et al., 2010). Secondly, while initial experiments were overemphasizing the role of syntaxin 1 and synaptobrevin 2 TMDs in overcoming the energy barrier for fusion (Fdez, Martínez-Salvador, Beard, Woodman, \& Hilfiker, 2010; Grote, Baba, Ohsumi, \& Novick, 2000; Han, Wang, Bai, Chapman, \& Jackson, 2004), recent data indicate that TMDs might be critical for fast, evoked membrane fusion, whereas for spontaneous fusion the structural requirements for membrane anchorage appear to be less strict (Zhou, Bacaj, Yang, Pang, \& Südhof, 2013). Interestingly, disruption of SNARE clusters by bioactive molecules such as anesthetics reduces the release capacities of chromaffin cells (Herring et al., 2011; Herring, Xie, Marks, \& Fox, 2009; Xie et al., 2013). Clustered SNAREs are in dynamic exchange with the surrounding membrane, and diffusion of SNARE molecules between clusters is rather high (Barg, Knowles, Chen, Midorikawa, \& Almers, 2010; Knowles et al., 2010; 
Sieber et al., 2007). The precise structure of the fusion site remains to be established. As discussed above, syntaxin clusters may serve as docking sites but the assembly of fusion competent SNARE complexes may take place adjacent to the clusters (Bar-On et al., 2012; Gandasi \& Barg, 2014; Rickman, $\mathrm{Hu}$, Carroll, \& Davletov, 2005).

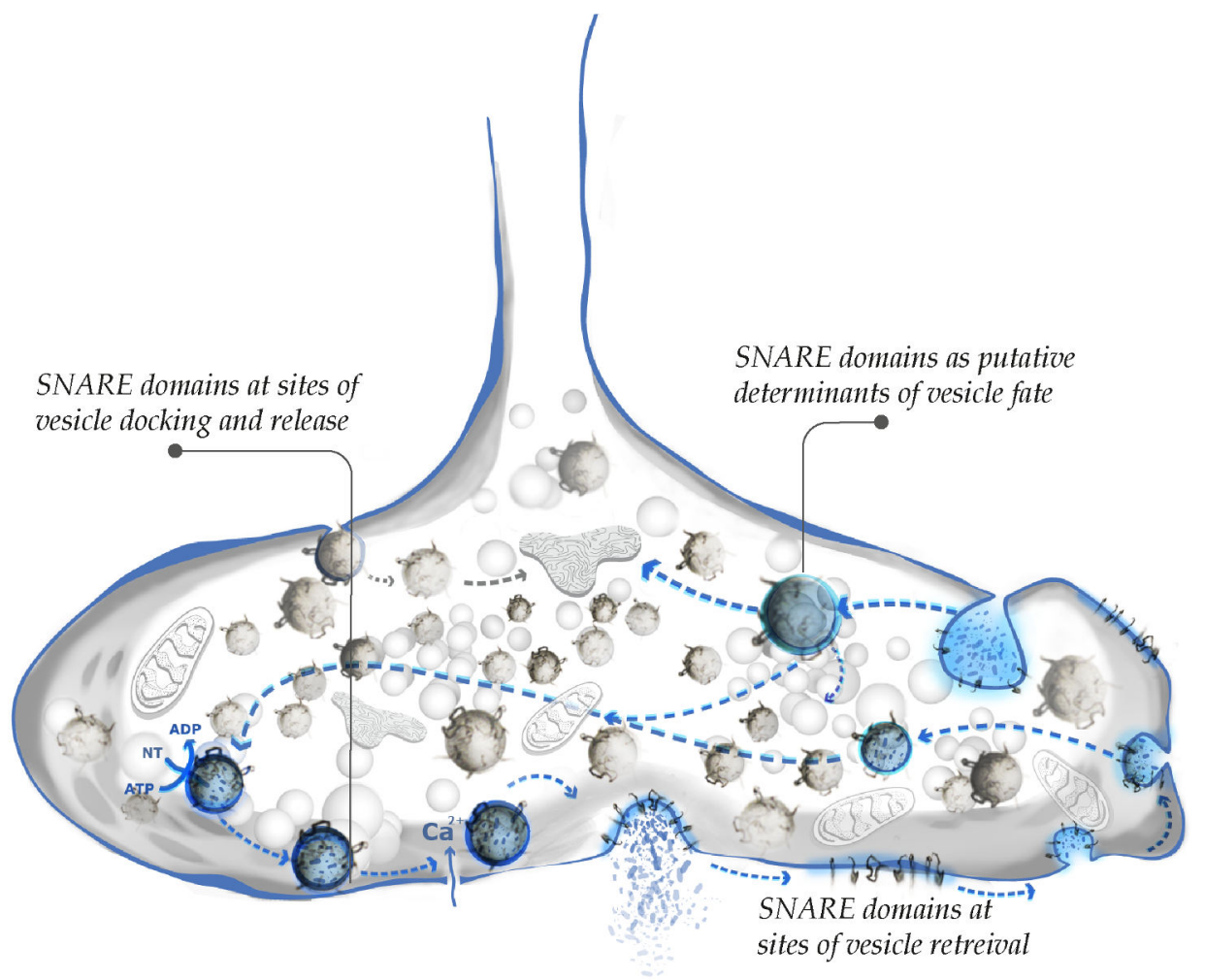

Figure 1.18. Scheme of the synaptic vesicle cycle. Details of the cycle are explained in the text. SNARE domains are indicated to play a role in vesicle docking. After fusion SNAREs either remain clustered or are re-clustered prior to endocytosis. During endocytosis (especially bulk retrieval) SNAREs may act as markers of membrane integrity and determine the subsequent sorting. Adapted from Milovanovic \& Jahn, 2015.

SNARE clustering may be important for endocytotic retrieval of vesicles. Neurotransmitter release is a rapid and repetitive process. In order to maintain membrane balance vesicle fusion and fission have to be tightly spatially and temporally coupled (Figure 18). During endocytosis, vesiclespecific proteins are selectively retrieved while plasma membrane residents are excluded. Even during sustained, high activity, the composition of synaptic vesicles needs to remain constant. While some flexibility may be tolerated for abundant proteins such as synaptobrevin 2 ( 70 copies/vesicle) 
some of the functionally essential proteins are present only $1-2$ copies/vesicle, e.g. the vacuolar ATPase required for neurotransmitter uptake (Takamori et al., 2006). Using STED microscopy, Willig et al. proposed that SV proteins remain clustered after exocytosis (Willig, Rizzoli, Westphal, Jahn, \& Hell, 2006). Alternatively, SV proteins may be sorted and re-clustered prior to endocytosis (Hua et al., 2011). Interestingly, a study that combined the electron microscopy and the STED nanoscopy showed that synaptic vesicle proteins such as synaptotagmin remain clustered even within the early endosome, thus being a marker for synaptic vesicle retrieval (Hoopmann et al., 2010).

Generally, there appear to be at least two main pathways for vesicle endocytosis: (i) slow, clathrin-mediated endocytosis (CME), and (ii) fast, mostly clathrin-independent, endocytosis. CME has been extensively studied (Dittman \& Ryan, 2009; Jung \& Haucke, 2007). The relatively slow kinetics of CME ( 20 seconds) cannot fully explain fast vesicle turnover at the synaptic bouton (Gandhi \& Stevens, 2003; Heuser \& Reese, 1973). Using a combination of optogenetics and high-pressure freezing electron microscopy, Jorgensen and colleagues showed that a second type of endocytosis co-exists in neurons that can be very rapid ( $\sim 30 \mathrm{~ms})$ but is likely to be less accurate than CME, resulting in endocytotic membrane vesicles larger than SV (S. Watanabe, Lehmann, et al., 2014a; S. Watanabe, Liu, et al., 2013a; S. Watanabe, Rost, et al., 2013b). Apart from speed, the availability of endocytotic machinery might be the limiting step in CME during the sustained SV release. Indeed, quantitative analysis of the synaptic bouton showed that there are about five folds less endocytotic than exocytotic proteins (Wilhelm et al., 2014). This problem may be overcome by fast, bulk endocytosis that requires fewer proteins to be involved in vesicle engulfing (S. Watanabe, Rost, et al., 2013b).

It is still debated to which extent endocytosed vesicles need to pass through an additional endosomal sorting step before re-entering the SV pool. It is conceivable that the fate of the endocytosed membrane is determined by its protein and lipid components (Rizzoli, 2014). Shortly after exocytosis the protein content of the synaptic vesicle either remains clustered (Willig et al., 2006), or it diffuses in the plane of the membrane which is followed by immediate re-clustering (Hua et al., 2011; Wienisch \& Klingauf, 2006). Specific adaptor proteins such as AP2, stonin and AP 180 specifically bind to synaptic 
vesicle proteins such as synaptobrevin 2 (AP 180) (Granseth, Odermatt, Royle, \& Lagnado, 2006) or synaptotagmin 1 (AP2, stonin) (Collins, McCoy, Kent, Evans, \& Owen, 2002; Jung et al., 2007), ensuring their clustering in a coated pit (Glyvuk et al., 2010). It is conceivable that a clathrin-coated vesicle separating from the plasma membrane matches the membrane composition of synaptic vesicles (as already suggested earlier; Maycox, Link, Reetz, Morris, \& Jahn, 1992), allowing for immediate re-use after uncoating without an intermediate sorting step. In contrast, it is highly unlikely that vesicles retrieved from the plasma membrane by ultrarapid endocytosis are sorted with similarly high accuracy (Watanabe, Rost, et al., 2013b). It is conceivable that these vesicles need to "proof-read" by cytoplasmic factors after endocytosis (Figure 17). If the protein and lipid content of such an endocytosed vesicle meet the requirements for a functional synaptic vesicle, the vesicle might be loaded with NT and can be immediately used for the next round of the release. Otherwise, the vesicle is targeted to recycling endosomes for further sorting (Watanabe, Trimbuch, et al., 2014b). The precise sorting mechanism is far from understood and the sorting signals involved in vesicle recycling and the maintenance of the vesicle identity still need to be identified.

\subsection{AIMS OF THIS THESIS}

As explained above, the presence of many structurally and funstionally different domains in the plasma membrane with distinct compositions cannot be explained by only one, or just a few, parameters. Therefore, during my graduate studies, I worked on a broad range of concepts that consider electrostatic interactions, protein-protein (homo- and heterotypic) interactions, and hydrophobic interactions between bilayer core and protein transmembrane domains as parameters that contribute to segregation of proteins and lipids in distinct domains. Using SNAREs as an exquisite model to look at different clustering mechanisms, I focused on three specific topics.

First, I looked at the effect of hydrophobic mismatch on SNARE clustering. The crystal structure of the neuronal SNARE complex revealed that the TMD of syntaxin 1 may be too short to span the entire plasma 
membrane (Stein et al., 2009). In comparison to syntaxin 1 (involved in calcium regulated exocytosis), syntaxin 4 (involved in the constitutive exocytosis) has a slightly (1-2 residues) longer TMD (Fig. 1.19). As shown previously, the length of the TMDs plays an important role in trafficking different syntaxins to the particular organelles (Watson \& Pessin, 2001). However, for syntaxin 1 and 4 the TMD lengths are respectively 2 and 3 amino acids shorter than the average TMD length of other plasma membrane proteins (Sharpe et al., 2010). Therefore, I addressed if these shorter TMDs contribute to clustering of syntaxin isoforms in the plasma membrane. Also, I examined if the 1-2 residue difference in the TMD length between syntaxins 1 and 4 attribute to the lateral segregation of these SNAREs into distinct domains.
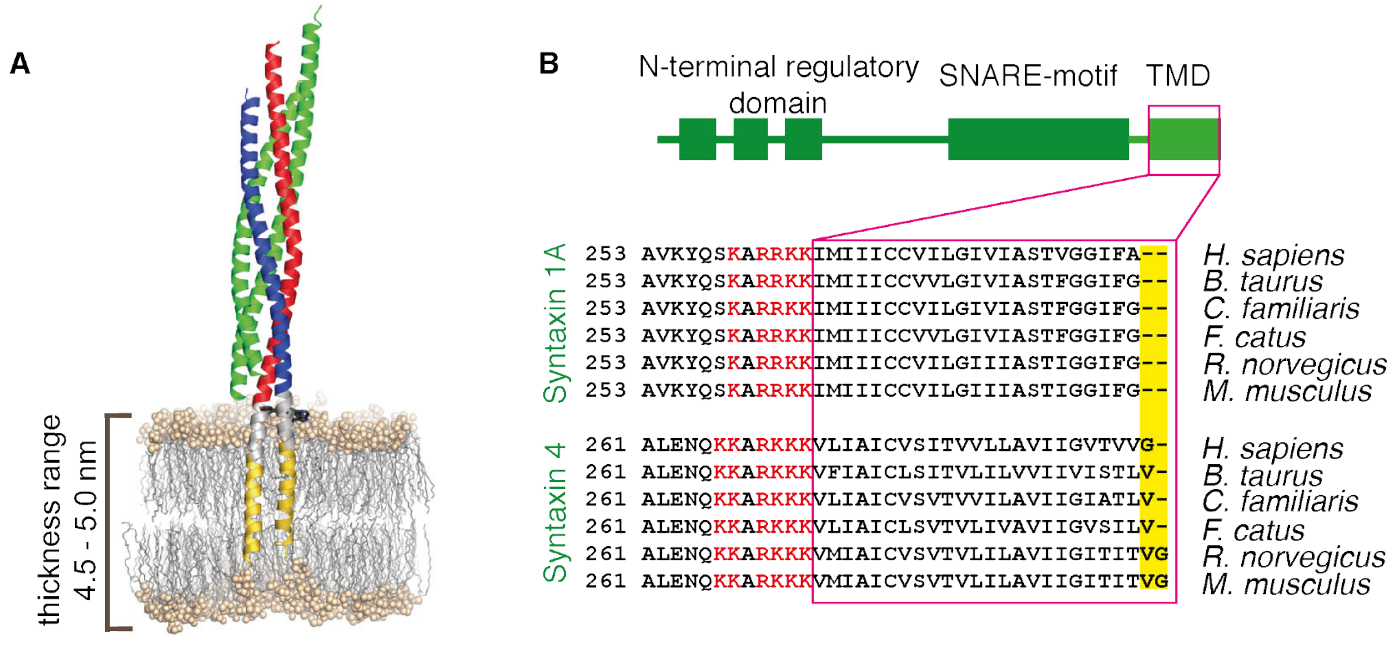

Figure 1.19. Plasma membrane SNAREs may be too short to span the entire membrane. A. Crystal structure of the full length neuronal SNARE complex. Neither the TMD of syntaxin 1 nor synaptobrevin 2 are long enough to span the average thickness of the plasma membrane. B. Sequence alignment of syntaxin 1 and syntaxin 4 with the TMDs marked in pink and polybasic patch highlighted in red. Adapted from Stein et al., 2009 and Milovanovic et al., 2015.

Second, I studied the interplay between different clustering mechanisms on SNARE domain formation. Homologous protein-protein interactions between SNARE motifs have been convincingly shown to cluster syntaxin 1 and 4 into distinct domains (Sieber et al., 2006). In addition, both 
syntaxin 1 and 4 contain the polybasic amino acid patch juxtaposed to the TMD that interacts with $\mathrm{PI}(4,5) \mathrm{P}_{2}$ in the plasma membrane (van den Bogaart et al., 2011). In this context, I addressed the contribution of hydrophobic mismatch on syntaxin clustering by varying the bilayer thickness in the presence of $\mathrm{PI}(4,5) \mathrm{P}_{2}$. I further looked at several mutants where the difference in TMD length between syntaxin 1 and 4 was either eliminated or enhanced. Together these data show the effect of hydrophobic mismatch in the context of the plasma membrane.

Third, I wanted to decipher how calcium ions affect syntaxin clusters. For SNAREs, it has been shown that an increased calcium concentration promotes formation of larger domains (Zilly et al., 2011). For the proteins with a dominant negative charge at the cytosolic surface this is explained by direct ionic interactions between calcium and the carboxyl groups of the amino acid side chains or C-terminus. However, syntaxin has a polybasic patch at the cytosolic surface and calcium is still able to induce $\sim 200 \mathrm{~nm}$ sized domains even of truncated syntaxin 1 mutants with anionic residues deleted. In this part I show that calcium acts as a charge bridge that connects multiple syntaxin 1 proteins into larger, mesoscale domains through $\mathrm{PI}(4,5) \mathrm{P}_{2}$

Overall this Thesis emphasizes that multiple mechanisms contribute to the lateral distribution of proteins and lipids in clusters at the plasma membrane. It is becoming apparent that these plasma membrane domains can represent local hot spots that are essential for the functional segregation of distinct cellular processes and can also contribute to vesicle tethering at the plasma membrane (see for instance Honigmann et al., 2013), discussed in details in Chapter 1.4). 


\section{MATERIALS AND METHODS}

\subsection{PEPTIDES AND LIPIDS}

Peptides used for the reconstitution experiments were synthesized using Fmoc solid phase synthesis by the group of Prof. Ulf Diederichsen, University of Göttingen. The fluorescent dyes Atto647N NHS-ester (Atto-Tec) and Rodamine red succinimidyl ester (Life Technologies) were coupled to the N-termini of syntaxin transmembrane domain (sx TMD). The detailed synthesis is described in the Appendix 11.1.

Precisely, the peptides used were:

(i) syntaxin 1 TMD (residues 266-288; sx-1 TMD) from Rattus norvegicus;

(ii) syntaxin 4 TMD (residues 262-297; sx-4 TMD) from Homo sapiens;

(iii) syntaxin 1 TMD mutant (sx-1 TMD with the following mutations: M267A, C271A, I279A);

(iv) syntaxin 1 polybasic patch mutant (sx-1 TMD mutant with the following mutations: K264A, K265A).

Lipids used in this study are:

(a) C18:1 PC (1,2-dioleoyl-sn-glycero-3-phosphocholine),

(b) C14:1 PC(1,2-dimyristoleoyl-sn-glycero-3-phosphocholine),

(c) C16:1 PC (1,2-dipalmitoleoyl-sn-glycero-3-phosphocholine),

(d) C20:1 PC (1,2-dieicosenoyl-sn-glycero-3-phosphocholine),

(e) brain $\mathrm{PI}(4,5) \mathrm{P}_{2}\left(\mathrm{PI}(4,5) \mathrm{P}_{2}\right.$ isolated from porcine brain),

(f) $\operatorname{doPI}(4,5) \mathrm{P}_{2}$ (1,2-dioleoyl-sn-glycero-3-phosphatidyl-(1'-myo-inositol-4', 5' bisphosphate)),

(g) brain PS (L- $\alpha$-phosphatidylserine isolated from porcine brain),

(h) cholesterol. Lipids $a-h$ were purchased from Avanti Polar Lipids.

(i) The lipophilic fluorescent probe $\mathrm{DiO}$ (3,3'-dilinoleyloxacarbocyanine perchlorate) was from Life Technologies.

(j) doPI $(4,5) \mathrm{P}_{2}$ labeled with Atto647N at the SN2 position.

(k) C16-ceramide labeled with Atto590. Lipids $j$ and $k$ were kindly provided by Dr. Vladimir Belov of the Max Planck Institute for Biophysical Chemistry and described in (Kolmakov et al., 2010). 


\subsection{MEMBRANE RECONSTITUTIONS}

\subsubsection{Polymer supported membranes}

Polymer supported membranes are used to generate a single lipid bilayer on glass cover slides used in microscopy (Fig. 2.1; Roder et al., 2011). First, the surface of glass slides was cleaned using fresh piranha solution (mixture of concentrated sulfuric acid and hydrogenperoxide in a 2:1 volume ratio) for $20 \mathrm{~min}$. The cleaned coverslips were extensively rinsed with distilled water in order to entirely remove the piranha solution and bath sonicated in distilled water for $5 \mathrm{~min}$. Coverslips were then dried with a stream of nitrogen gas.

Activation of the glass surface was done using pure GOPTS ((3glycidyloxypropyl)-trimetoxysilane; Sigma). The reaction was performed in a pyraniaclean dish by placing one coverslip to the bottom. One drop of GOPTS was added (carefully, with a syringe) and a 'sandwich' was made by positioning another cover slip on top of the GOPTS. The coverslips with the GOPTS were incubated for $50 \mathrm{~min}$ at $75^{\circ} \mathrm{C}$. This is a crucial step where the surface of the glass was activated. GOPTS introduces the highly reactive epoxide group at the glass surface by silanization. Meanwhile, $1 \mathrm{~g}$ (the tip of a spatula) of DAPEG (diaminopolyethylene glycol; Rapp Polymer Tubingen) was preheated for $10 \mathrm{~min}$ at $75^{\circ} \mathrm{C}$. DAPEG was taken out of the freezer before use and warmed to room temperature to prevent its condensation (especially important since it is a hygroscopic substance).

After activation the glass slides were washed with acetone to remove excess GOPTS and then placed on pre-melted DAPEG to incubate overnight at $75^{\circ} \mathrm{C}$. DAPEG has two functional amino groups at its ends. One amino group of DAPEG can interact with the epoxide groups at the glass surface leaving the amino group on other end of DAPEG free to react with the anchoring molecule. The phospholipid membranes were tethered to these amino groups by C16 acyl chains. For this purpose, the DAPEG coated glass slides were covered with $0.5 \mathrm{M}$ palmitic acid (Sigma) in DMSO and diisopropylcarbodiimide (Sigma) in a 2:1 volume ratio for $45 \mathrm{~min}$ incubation at room temperature. 
Finally, the membranes were successfully spin-coated on the coverslip (at $100 \mathrm{xg}$ ). For the spin-coating, I prepared a mixture with a 2:1 molar ratio of phospholipids to cholesterol, the lipid label (DiO, $0.01 \mathrm{~mol} \%$ ) and the sx-1 TMD labeled with Atto647N (Atto Tech) in a 1/10,000 molar ratio. Total concentration of spin-coated lipids was $1 \mathrm{mg} / \mathrm{ml}$ in chloroform. After spincoating the lipid film was rehydrated in 1,000 $\mu \mathrm{L}$ of $50 \mathrm{mM}$ HEPES buffer that contained $150 \mathrm{mM} \mathrm{KCl}$ (pH at 7.4).

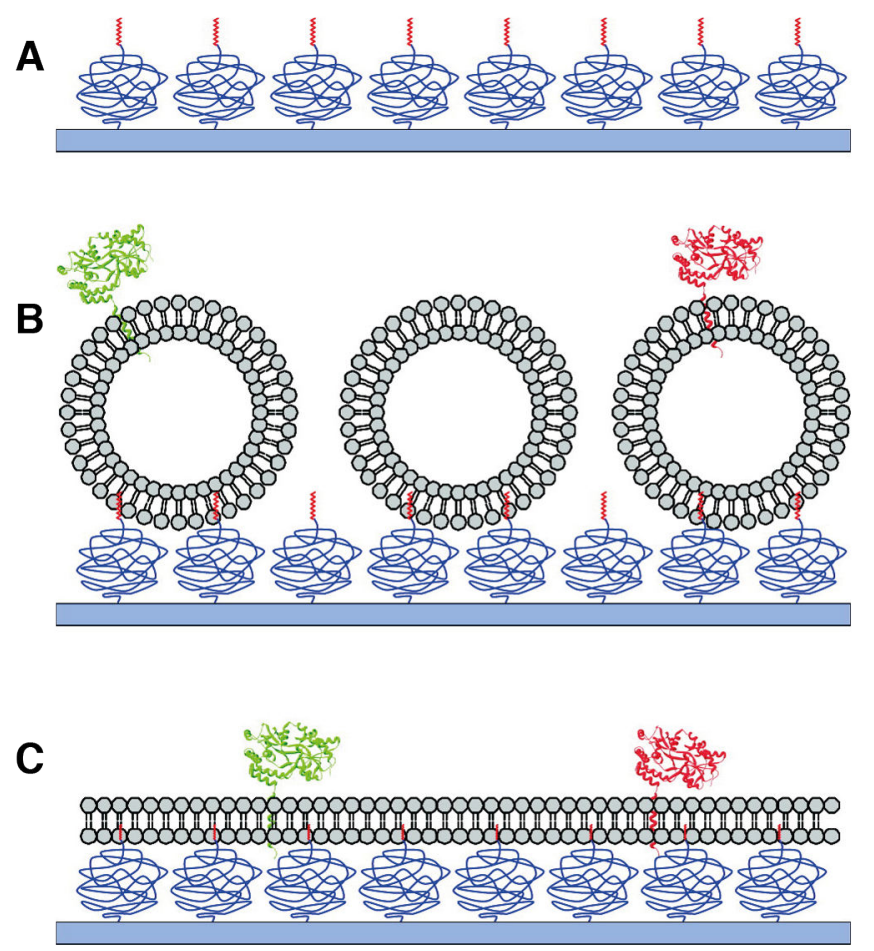

Figure 2.1. Scheme of the assay for preparation of PSM. A. Activation of the glass surface and functionalization with DAPEG (polymer, blue) and palmitic acid (anchoring molecule, red). B. Vesicles are loaded to the functionalized glass surface and captured by the palmitate group. C. After incubation and washing away the excess of vesicles, an extended polymer-supported bilayer is formed. Please note that this step I perform by spin-coating the vesicles on the activated glass surface. Adapted from Roder et al., 2011.

\subsubsection{Stacked lipid bilayers}

Glass cover slides used in microscopy were prepared by vigorous cleaning in ethanol as described in (Mennicke \& Salditt, 2002) and the supported lipid bilayer was generated by spin-coating. For the spin-coating (at $100 \mathrm{xg}$ ) we prepared a $40 \mathrm{mM}$ lipid mixture consisting of different lipid 
mixtures (indicated in each of the experiments) in chloroform:ethanol (1:1 volume mixture). The molar ratio of syntaxin TMDs to phospholipids was 1:10,000. After spin-coating $10 \mu \mathrm{L}$ of the lipid solution, the lipid film was rehydrated in $1 \mathrm{~mL}$ of $25 \mathrm{mM}$ HEPES buffer with $150 \mathrm{mM} \mathrm{NaCl}$ (pH at 7.4). The spin-coated cover slips were rehydrated in a sample chamber with 1,000 $\mu \mathrm{l}$ of buffer (25 mM HEPES, $150 \mathrm{mM} \mathrm{NaCl}$, pH 7.4). When indicated, calcium and EDTA were added directly into the sample chamber and the samples were mixed by gentle pipetting prior to STED imaging.

\subsubsection{Large unilamellar vesicles}

Large unilamellar vesicles (LUV) were prepared from PC of different acyl-chain lengths (C14:1, C16:1, C18:1, and C20:1) with or without $30 \mathrm{~mol} \%$ cholesterol and/or $1 \mathrm{~mol} \% \mathrm{PI}(4,5) \mathrm{P}_{2}$. Lipid mixtures were prepared at atal concentration of approximately $30 \mathrm{mM}$ lipids in chloroform as described in (Schuette et al., 2004). After removal of the chloroform with a rotary evaporator (Buchi Rotavapor R-124), the lipid film was resuspended to $40 \mathrm{mM}$ in methanol and fluorescently labeled peptides were added in 2,2trifluoroethanol (protein-to-lipid ratios are given at each of experiments). The organic solvents were then evaporated and the dried lipid film resuspended to $8 \mathrm{mM}$ total lipid concentration in $25 \mathrm{mM}$ HEPES buffer with $150 \mathrm{mM} \mathrm{KCl}$ ( $\mathrm{pH}$ 7.4) unless otherwise indicated (i.e. $\mathrm{NaCl}$, ATP, $\mathrm{MgCl}_{2}$ ). Multilamellar vesicles, which are generated by resuspending the lipid film, were then extruded through polycarbonate filters with $100 \mathrm{~nm}$ pore diameter (Fig. 2.2) (Avanti Polar Lipids). Vesicle sizes were confirmed by dynamic light scattering (Wyatt Technologies). 


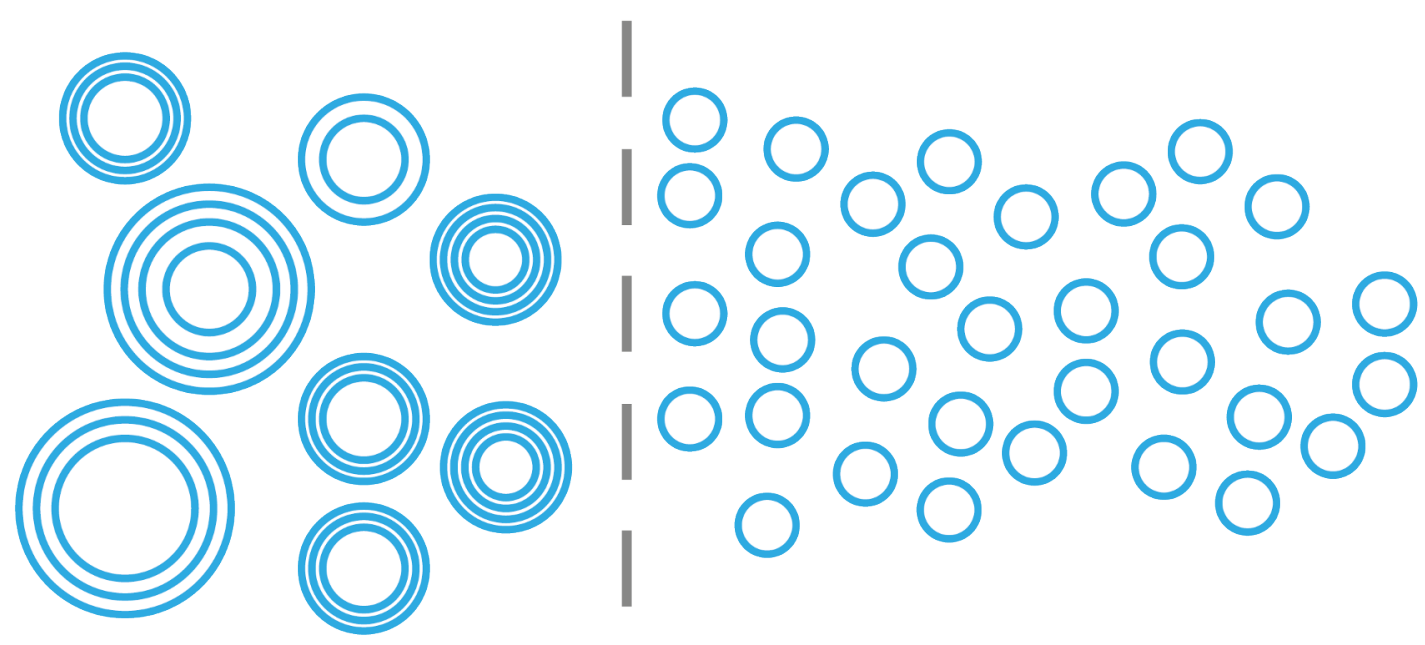

Figure 2.2. Scheme of LUVs generation by extrusion. The mixture of multilamellar vesicles is pushed through the polycarbonate filter with pore size of $100 \mathrm{~nm}$ diameter.

\subsubsection{Giant unilamellar vesicles}

Large $\mu \mathrm{m}$-range diameter giant unilamellar vesicles (GUVs) are suitable for directly observing phase separation by microscopy. Their large diameter also imply low curvature, hence all the effects observed are decoupled from curvature stress. Two main methods for GUV formation are passive swelling and electroformation (Doeven et al., 2005; Ramadurai et al., 2009; van den Bogaart et al., 2011). Passive swelling relies on the repulsion between charged polar heads of the phospholipids, whereas electroformation uses an AC electric field that interacts with the dipole moments of the lipid molecules and causes its agitation. The precise lipid mixtures used are indicated for each of the experiments. DiO was used for fluorescent labeling of the lipid phase $(0.01 \mathrm{~mol} \%)$ and sx-1 TMD was used in 1:1,000 protein-tolipid ratio. For passive swelling, $1 \mu \mathrm{L}$ of the sx-1 TMD/lipid mixture was dried on a preheated glass slide at $55^{\circ} \mathrm{C}$ and rehydrated in $100 \mu \mathrm{L}$ of prewarmed ddH20 for 20 min. Electroformation was performed by drying $1 \mu \mathrm{L}$ sample on an ITO-coated glass slide at $55^{\circ} \mathrm{C}$. A silicone O-ring was then placed around the dried sample on the glass slide and rehydrated with $500 \mu \mathrm{L}$ of pre-warmed ddH20. The rehydration suspension was positioned between two ITO-coated glasses for current conduction. Electro-formation was performed for $45 \mathrm{~min}$ at 1.2 V/10 Hz (Function Generator FG250D, Conrad). 


\subsection{DYNAMIC LIGHT SCATTERING}

Dynamic light scattering (DLS) is a technique used to determine the size distribution of particles in suspension (Berne \& Pecora, 2000). Here monochromatic light (i.e. laser) goes through the polarizer to the sample. Particles in the sample diffract the light in all directions and this light goes through the second polarizer to the photomultiplier.

The speckle pattern at the photomultiplier (light regions: positive interference, dark region: negative interference) is analyzed for the intensity change over time. From the autocorrelation function of the time dependent intensity change, diffusion coefficients (D) are calculated. The Stokes-Einstein relationship: $D_{h}=k_{B} T / 3 p \eta D_{t}\left(D_{h}\right.$ is the hydrodynamic diameter (i.e. particle size); $k_{B}$ is the Boltzman constant; $\eta$ the solvent viscosity; $D_{t}$ is the translational diffusion coefficient obtained by DLS) was used to estimate the hydrodynamic radius, $\mathrm{D}_{\mathrm{h}}(\mathrm{nm})$. In my case diffusion coefficients were measured at $25^{\circ} \mathrm{C}$ at a laser wavelength of $830 \mathrm{~nm}$ and scattering angle of $90^{\circ}$ using a DynaPro Titan DLS.

To illustrate the application of DLS for determining the vesicle size, the distribution of LUVs with an average vesicle radius of $54.5 \pm 7.3 \mathrm{~nm}$ is shown in Fig. 2.3 (preparation of LUVs according to the protocol in 2.2.3; three independent reconstitutions, ten measurements each).

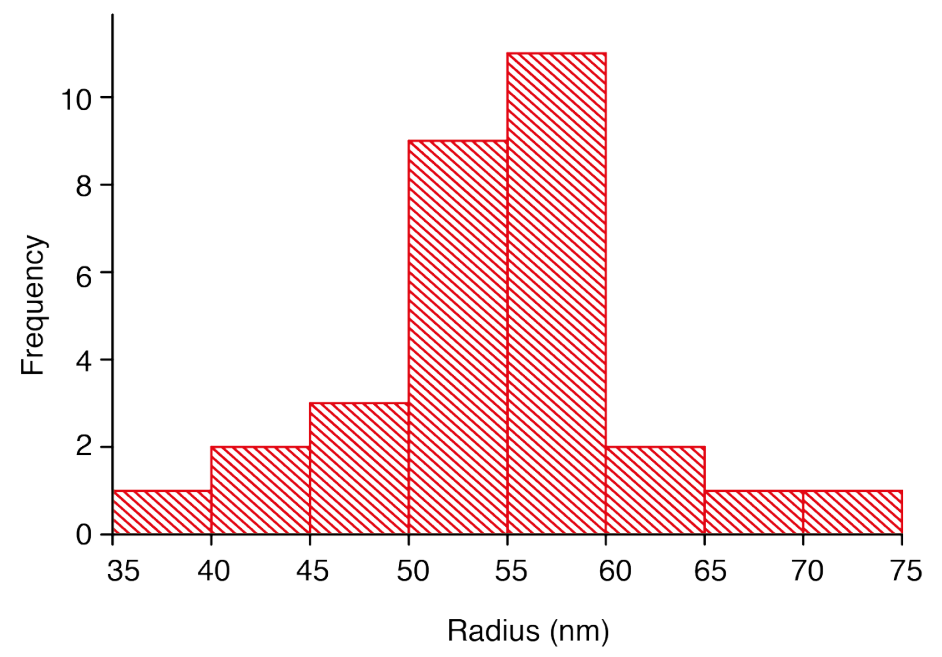

Figure 2.3. DLS measurement of the average radius $(54.5 \pm 7.3 \mathrm{~nm})$ of LUVs prepared by extrusion procedure described in Materials and Methods 2.2.3. Data are generated from three independent reconstitutions, ten measurements each. 


\subsection{CELL CULTURE AND IMMUNOFLUORESCENCE}

In this study, I used the pheochromocytoma cell line PC12 from Rattus norvegicus (Greene \& Tischler, 1976; Heumann, Kachel, \& Thoenen, 1983). Lipofectamin LTX reagents from Life Technologies were used for transfection and cells were analyzed 24 hours posttransfection. Native membrane sheets were generated by gentle sonication (Sieber et al., 2007; van den Bogaart et al., 2011) and sonication buffer contained $20 \mathrm{mM}$ K-HEPES pH 7.4, $120 \mathrm{mM}$ K-gluconate, $20 \mathrm{mM}$ K-acetate, $2 \mathrm{mM}$ ATP and $0.5 \mathrm{mM}$ DTT (note: fresh ATP and DTT were added prior to sonication) (Fig. 2.4).

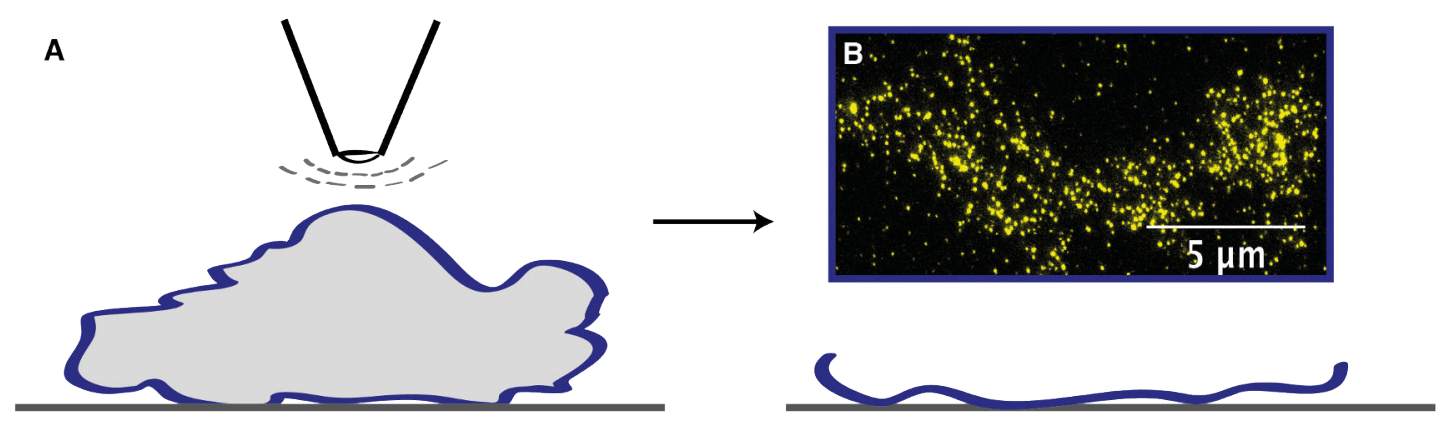

Figure 2.4. Preparation of cell sheets. A. Scheme of preparation of cell sheets using a short sonification pulse (for details pleas see text). B. Example of PC12 membrane sheet immunostained against syntaxin 1 (HPC-1 IgG1; shown in yellow). The inner leaflet of the plasma membrane is accessible for both immunostaining and imaging.

Primary antibodies used for immunohistochemistry experiments were:

1. syntaxin 1 HPC-1 IgG1 (Sigma, clone HPC-1);

2. syntaxin 1 rabbit polyclonal antiserum (Synaptic Systems, cat. number 110.302);

3. syntaxin 4 mouse monoclonal IgG1 (Synaptic Systems, clone number 139.2, cat. number 110.041);

4. mouse IgM antibodies against $\mathrm{PI}(4,5) \mathrm{P}_{2}$ (Echelon, clone Z-A045);

5. mouse monoclonal IgG2a anti-mCherry (Abcam, clone 1C51, cat. number ab125096);

6. rabbit polyclonal anti-EGFP (Abcam, cat. number ab290). 
Secondary antibodies against IgG and IgM were labeled with Alexa Fluor 488C5-maleimide (Life Technologies) or KK114-maleimide (gift from Vladimir Belov, MPI-BPC, Göttingen, Germany, described in (Honigmann et al., 2013)).

For transfection of PC12 cells we used synthetic chimeric constructs (Genscript) in the KpnI-HindIII restriction sites of pCEP4. The sequences for syntaxin 1A (sequence from Rattus norvegicus 262-297) N-terminally tagged with mCherry or mEGFP are given in Table 1 . The constructs coding for mCherry-tagged sx-1 TMD-IFG (residues 257-285) and sx-1 TMD+VG (residues 257-288 with two additional amino acids at the C-terminus: V289, G290) were generated from the wild-type construct by Quick Change mutagenesis (Agilent Technologies).

Table 1 | Syntaxin 1A and syntaxin 4 sequences used for PC12 cell transfections.

\begin{tabular}{|l|}
\hline 1. Syntaxin 1A (sequence from Rattus norvegicus, residues 257-288) \\
N-terminally tagged with mCherry \\
\hline 5'ATGGTGAGCAAGGGCGAGGAGGACAACATGGCCATCATCAAGGAGTTCATGAGGT \\
TCAAGGTGCACATGGAGGGCAGCGTGAACGGCCACGAGTTCGAGATCGAGGGCGAG \\
GGCGAGGGCAGGCCCTACGAGGGCACCCAGACCGCCAAGCTGAAGGTGACCAAGG \\
GCGGCCCCCTGCCTTCGCCTGGGACATCCTGAGCCCCCAGTTCATGTACGGCAGCA \\
AGGCCTACGTGAAGCACCCCGCCGACATCCCCGACTACCTGAAGCTGAGCTTCCCCG \\
AGGGCTTCAAGTGGGAGAGGGTGATGAACTTCGAGGACGGCGGCGTGGTGACCGTG \\
ACCCAGGACAGCAGCCTGCAGGACGGCGAGTTCATCTACAAGGTGAAGCTGAGGGG \\
CACCAACTTCCCCAGCGACGGCCCCGTGATGCAGAAGAAGACCATGGGCTGGGAGG \\
CCAGCAGCGAGAGGATGTACCCCGAGGACGGCGCCCTGAAGGGCGAGATCAAGCAG \\
AGGCTGAAGCTGAAGGACGGCGGCCACTACGACGCCGAGGTGAAGACCACCTACAA \\
GGCCAAGAAGCCCGTGCAGCTGCCCGGCGCCTACAACGTGAACATCAAGCTGGACA \\
TCACCAGCCACAACGAGGACTACACCATCGTGGAGCAGTACGAGAGGGCCGAGGGC \\
AGGCACAGCACCGGCGGCATGGACGAGCTGTACAAGGGCGGCCACAGGTGGATCTA \\
CCAGAGCAAGGCCAGGAGGAAGAAGATCATGATCATCATCTGCTGCGTGATCCTGG \\
GCATCGTGATCGCCAGCACCGTGGGCGGCATCTTCGCC3'
\end{tabular}

2. Syntaxin 1A (sequence from Rattus norvegicus, residues 257-288) N-terminally tagged with mEGFP

5'GGTACCATGGTGAGCAAGGGCGAGGAGCTGTTCACCGGCGTGGTGCCCATCCTGGT GGAGCTGGACGGCGACGTGAACGGCCACAAGTTCAGCGTGAGCGGCGAGGGCGAGG GCGACGCCACCTACGGCAAGCTGACCCTGAAGTTCATCTGCACCACCGGCAAGCTGC CCGTGCCCTGGCCCACCCTGGTGACCACCCTGACCTACGGCGTGCAGTGCTTCAGCA GGTATCCCGACCACATGAAGCAGCACGACTTCTTCAAGAGCGCCATGCCCGAGGGCT ACGTGCAGGAGAGGACCATCTTCTTCAAGGACGACGGCAACTACAAGACCAGGGCC GAGGTGAAGTTCGAGGGCGACACCCTGGTGAACAGGATCGAGCTGAAGGGCATCGA CTTCAAGGAGGACGGCAACATCCTGGGCCACAAGCTGGAGTACAACTACAACAGCC ACAACGTGTACATCATGGCCGACAAGCAGAAGAACGGCATCAAGGTGAACTTCAAG ATCAGGCACAACATCGAGGACGGCAGCGTGCAGCTGGCCGACCACTACCAGCAGAA 
CACCCCCATCGGCGACGGCCCCGTGCTGCTGCCCGACAACCACTACCTGAGCACCCA GAGCAAGCTGAGCAAGGACCCCAACGAGAAGAGGGACCACATGGTGCTGCTGGAGT TCGTGACCGCCGCCGGCATCACCCTGGGCATGGACGAGCTGTACAAGGGCGGCCAC AGGTGGATCTACCAGAGCAAGGCCAGGAGGAAGAAGATCATGATCATCATCTGCTG CGTGATCCTGGGCATCATCATCGCCAGCACCATCGGCGGCATCTTCGGCTAGTAAAA GCTT3'

3. Syntaxin 4 (sequence from Rattus norvegicus, residues 262-297) N-terminally tagged with mEGFP.

5’ATGGTGAGCAAAGGCGAAGAACTGTTTACCGGCGTGGTGCCGATTCTGGTGGAACT GGATGGCGATGTGAACGGCCATAAATTTAGCGTGAGCGGCGAAGGCGAAGGCGATG CGACCTATGGCAAACTGACCCTGAAATTTATTTGCACCACCGGCAAACTGCCGGTGC CGTGGCCGACCCTGGTGACCACCCTGACCTATGGCGTGCAGTGCTTTAGCCGCTATCC GGATCATATGAAACAGCATGATTTTTTTAAAAGCGCGATGCCGGAAGGCTATGTGCA GGAACGCACCATTTTTTTTAAAGATGATGGCAACTATAAAACCCGCGCGGAAGTGAA ATTTGAAGGCGATACCCTGGTGAACCGCATTGAACTGAAAGGCATTGATTTTAAAGA AGATGGCAACATTCTGGGCCATAAACTGGAATATAACTATAACAGCCATAACGTGTA TATTATGGCGGATAAACAGAAAAACGGCATTAAAGTGAACTTTAAAATTCGCCATAA CATTGAAGATGGCAGCGTGCAGCTGGCGGATCATTATCAGCAGAACACCCCGATTGG CGATGGCCCGGTGCTGCTGCCGGATAACCATTATCTGAGCACCCAGAGCAAACTGAG CAAAGATCCGAACGAAAAACGCGATCATATGGTGCTGCTGGAATTTGTGACCGCGGC GGGCATTACCCTGGGCATGGATGAACTGTATAAAGGCGGCCATCGCTGGATTGCGCT GGAAAACCAGAAAAAAGCGCGCAAAAAAAAAGTGCTGATTGCGATTTGCGTGAGCA TTACCGTGGTGCTGCTGGCGGTGATTATTGGCGTGACCGTGGTGGGC3’

\subsection{SUPERRESOLUTION STIMULATED EMISSION DEPLETION (STED) NANOSCOPY}

Due to the wave-properties of light, it is not possible to focus it to an infinitely small single point. The focus intensity functions of fluorophores within a certain minimal distance will overlap and cannot be well distinguished from each other. In this context, resolution is the minimal distance at which a microscope can distinguish two fluorophores. Resolution is often confused with magnification or precision (i.e. calculating the position of the center of a single PSF). In the 19 $9^{\text {th }}$ century, the famous Abbe's principle was formulated explaining that the resolution limitation is a direct consequence of diffraction (Abbe, 1873). In conventional microscopy, the resolution is determined by the wavelength applied, the refractive index and the semiaperture of the objective (discussed in Hell, 2007). Since the shortest wavelength used for optical microscopy is around $400 \mathrm{~nm}$ (near-UV) and the largest semiaperture in objectives reaches $68^{\circ}$, the Abbe's principle shows that the best resolution accessible with conventional microscopy is around $180 \mathrm{~nm}$. 
This resolution is even lower in the axial direction of light propagation, where the resolution reaches only about $500 \mathrm{~nm}$.

The realization that fluorescence molecules can exist in two states (for instance, on-state: fluorescent, off-state: non-fluorescent, dark) was the key to surpass the diffraction barrier (Hell \& Kroug, 1995; Hell \& Wichmann, 1994; Klar, Jakobs, Dyba, Egner, \& Hell, 2000). In STED microscopy, a focused excitation laser beam excites fluorophores (Fig. 2.5A). In the very short time $(\sim \mathrm{ns})$ where the excited electrons are occupying the vibrational state of the higher energy level, a second so-called depletion laser provides light with a wavelength that matches the transition from the excited $\left(S_{1}\right)$ to the ground $\left(S_{0}\right)$ state which forces the stimulated emission of photons (Fig. 2.5B). This stimulated depletion (STED) laser is red shifted, so that it does not excite the fluorophores in the ground state.

If the intensity of the depletion beam is larger, the higher is the probability that an electron will be pushed down to the $S_{0}$ state when it reaches the excited state. In this case, obviously there would be no signal detected if the depletion laser were applied to the entire specimen. In STED microscopy, however, the depletion laser first passes through a phase plate to convert the shape of the focused profile into the shape of a ring (sometimes also referred to as a doughnut; Klar et al., 2000). Since the center of this region has zero light intensity, fluorophores do not get switched off in this position and fluorescence induced by the excitation laser can be detected (Fig. 2.5C,D). These two paradigms: molecular switching (distinct states) and phase plate modulation of the depletion laser, are the bases of stimulated emission depletion (STED) nanoscopy.

The most important consideration when employing STED nanoscopy for biological applications (Hell, 2007) is the selection of appropriate dyes and good labeling strategies. The dye wavelengths should be suitable for STED, which means that the STED beam is sufficiently far with respect to the excitation maximum. Also, the lifetime of the excited state of fluorophore should be long enough to allow for stimulated depletion. Thus, careful selection of dyes is required in terms of photostability and spectral properties.

Throughout my studies, I used three different STED microscopes from the Department of NanoBiophotonics. The first was a homemade beamscanning microscopy setup (built by Dr. Veronika Mueller, Dr. Alf 
Honigmann and Dr. Christian Eggeling) (Mueller et al., 2011). The setup contained two pulsed diode excitation lasers at $488 \mathrm{~nm}$ and $633 \mathrm{~nm}$ (PicoQuant). Superresolution was achieved using a STED laser $(770 \mathrm{~nm}$ OPSL $\mathrm{CW}$, Coherent). In combination with a $2 \pi$ vortex phase plate (RPC Photonics, USA) and a $\lambda / 4$ plate the typical "doughnut" shaped focal intensity distribution with its central zero was produced. The emission filters were 540 $\pm 20 \mathrm{~nm}$ for the green channel and $670 \pm 30 \mathrm{~nm}$ for the red channel. We used a 100x oil objective with a 1.42 numerical aperture (Leica). The excitation intensity was between 5-8 $\mu \mathrm{W}$ at a diffraction limited diameter of $250 \mathrm{~nm}$ (for $633 \mathrm{~nm}$ excitation) and $190 \mathrm{~nm}$ (for $488 \mathrm{~nm}$ excitation). The average STED power was $180 \mathrm{~mW}$ at $770 \mathrm{~nm}$. The resolution in the STED channel was around $50 \mathrm{~nm}$ (determined from imaging fluorescent beads).

The second setup was a two-color STED setup (built by Fabian Göttfert) (Göttfert et al., 2013). This setup had pulsed excitation at $595 \mathrm{~nm}$ and $640 \mathrm{~nm}$. The fluorescence was collected from $600-640 \mathrm{~nm}$ and $660-720 \mathrm{~nm}$ by avalanche photo diodes (Micro Photon Devices, Italy). Superresolution was achieved using a STED laser $(775 \mathrm{~nm}, 20 \mathrm{MHz}$ pulsed fiber laser, IPG Photonics). Pulse energies from 3 to $8 \mathrm{~nJ}$ in the objectives back aperture yield a resolution of down to $30 \mathrm{~nm}$. Using the same STED beam for both dyes inherently ensures a colocalization accuracy far below the resolution limit. As we record both color channels quasi simultaneously we do not have to correct for drift or channel misalignment.

The third setup employed was a commercially available two-color STED setup (Abberior Instruments, Göttingen). This setup has two pulsed excitation lasers at $594 \mathrm{~nm}$ and $640 \mathrm{~nm}$. For superresolution a pulsed STED laser at $775 \mathrm{~nm}$ was used. The setup has a QUAD beam scanner (Abberior Instruments, Göttingen). A resolution of around $30 \mathrm{~nm}$ was obtained.

The data acquisition was done using ImSpector software (http://www.imspector.de). The density of clusters was analyzed using the particle analysis plugin in the Fiji software and the cluster correlation was obtained using Pearsons correlation analysis from the Fiji software tools (Schindelin et al., 2012). 


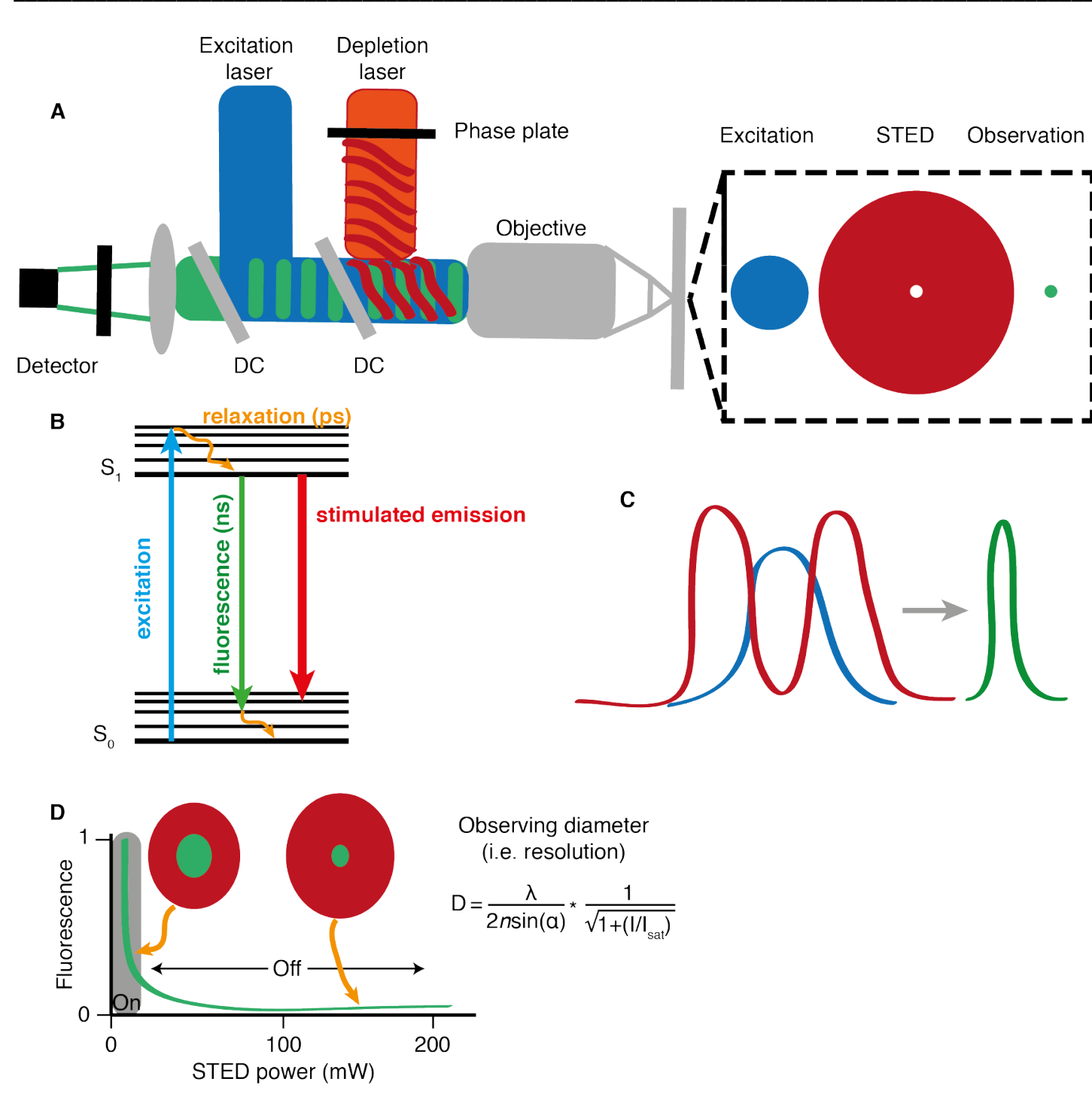

Figure 2.5. Principles of STED. A. Scheme of STED nanoscopy setup. An excitation laser activates all the fluorescent molecules that are in focus (blue). Superresolution is achieved by de-excitation (switching off) from $S_{1}$ to $S_{0}$ using a stimulated depletion (STED) light beam. The phase plate generates a ringshape STED beam, so that only fluorophores from the middle of the focus are excited and can be detected (green). B. Scheme of electronic states of a fluorophore. Upon absorption (blue arrow), the electrone goes to the excited $S_{1}$ state. After internal relaxation to the lowest vibrational energy, the electron (orange arrow) goes back to the ground $\left(\mathrm{S}_{0}\right)$ state emitting a photon (green arrow). However it is possible to force the electron to the ground state by stimulated emission (red arrow). C. Lateral cross section of the point spread functions (PSFs) at the focal plane of the excitation beam (blue), STED beam (red) and resulting fluorescence (green). Note that intensities of these functions are not to scale (i.e. the STED beam has order of magnitude higher intensity than the excitation beam). D. The probability to switch off 
fluorophores increases with increasing STED power, resulting in a reduced size of the excitation spot. Hence, by increasing the STED power (red) the volume with fluorescence emission (green) decreases. Modified Abbe's equation for STED microscopy: $\lambda$ is the wave length of the excitation light, $n$ is the refractive index of the medium, $\alpha$ is half-angle of the incoming light. $I$ is applied STED intensity, $I_{\text {sat }}$ is a threshold intensity needed to be applied in order to force the electrons from the excited to the ground state (it is dye and setup specific constant). Inspired by schemes from Hell, 2007 and Eggeling et al, 2015.

\subsection{FLUORESCENCE CORRELATION SPECTROSCOPY}

Fluorescence correlation spectroscopy (FCS) analyses fluorescence fluctuations in a focal spot generated by focused laser beams, the so-called confocal volume ( fL) (Magde, Elson, \& Webb, 1974; Ries \& Schwille, 2012). For FCS measurements I used a home-built confocal beam-scanning microscopy setup with two-color excitation by pulsed-diode lasers at $485 \mathrm{~nm}$ (pulse length 80 ps; LDH-P-635, PicoQuant) and $635 \mathrm{~nm}$ (pulse length 80 ps LDH-P-485B, PicoQuant). Emission filters were $540 \pm 20$ for the green channel and $670 \pm 30$ for the red channel (setup was built by Dr. Veronika Mueller, Dr. A. Honigmann and Dr. C. Eggeling, Department of NanoBiophotonics) (Honigmann et al., 2013; Mueller et al., 2011). Avalanche single photon counting detectors (APD, SPCM-AQR-13-FC, Perkin Elmer Optoelectronics) and a 100x oil objective with 1.42 NA (Leica Microsystems) were used.

Given that fluorescent molecules are sparse enough (i.e. highly diluted), the motion of these molecules through the confocal volume results in intensity fluctuations (Bacia, Haustein, \& Schwille, 2014; Ries \& Schwille, 2012). These intensity traces $F(t)$ are self-correlated in time to generate the autocorrelation curve $G(\tau)$ :

$$
G(\tau)=\frac{<(\partial F(t) * \partial F(t+\tau)>}{<F(t)^{2}>}
$$

where $F$ is the fluorescent intensity in time $t, \tau$ is the lag time and the angular brackets refer to time averaging. Within this work, I studied the lateral 
diffusion of sx-1 TMD and lipids in the membranes, hence the autocorrelation curve was fitted with the following two-dimensional diffusion model (Magde et al., 1974; Vukojević et al., 2005):

$$
G(\tau)=\left(\frac{1}{N *\left(1+\frac{t}{\tau d}\right)}\right) *\left(1+\frac{p}{1-p}\right) * e^{-\frac{t}{\tau t}}
$$

where $N$ is the average number of fluorescently labeled molecules in the detected volume and $p$ is the fraction of labeled molecules which convert to the dark triplet state, $\tau_{d}$ is the average transit time of molecule moving through the observation volume and $\tau_{T}$ is the average time the labeled molecule remain in the triplet state. Finally, the diffusion coefficient $D$ was calculated from the diffusion time $\tau_{d}$ :

$$
\tau d=\frac{\omega^{2}}{4 * D}(3)
$$

where $\omega$ is the lateral width of the focal volume where the measured fluorescence drops $\mathrm{e}^{2}$ times relative to the maximum and was 200 and $250 \mathrm{~nm}$ for $\mathrm{DiO}$ and Atto647N, respectively. In the experiments the bars represent the range of data obtained from two independent reconstitutions each consisting of four to seven measurements.
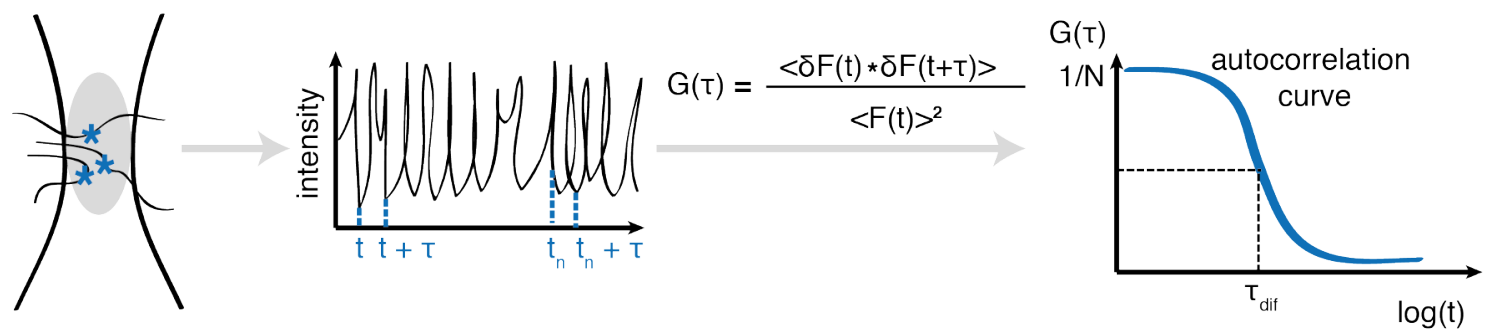

Figure 2.6. Principles of FCS. Fluorescent molecules diffuse through the detection volume of a confocal microscope generating signal fluctuations. The correlation curve is calculated as the autocorrelation of the intensity fluctuations. The amplitude of the curve corresponds to the inverse number of fluorescence molecules in the detection volume. The inflection point at the fitted curve decay is proportional to the diffusion time (i.e. time that a fluorescent molecule needs to diffuse through the detection volume). Adapted from Ries \& Schwille, 2012. 


\subsection{FÖRSTER RESONANCE ENERGY TRANSFER}

Förster resonance energy transfer (FRET) is a photophysical phenomenonon where within the very small distances a fluorophore can transfer its energy to a neighboring fluorophore and induce that fluorophore to fluoresce. The donor fluorophore that is excited by the initial excitation wavelength (provided by a controlled excitation source) contains electrons that are excited to a higher energy state. These electrons can relax to the ground state, and the energy released by their relaxation can result in fluorescence emission. In FRET, instead of these electrons returning to the ground state, they can transfer their energy to a nearby fluorophore. This fluorophore is termed the "acceptor" fluorophore, while the initial fluorophore is called the "donor" fluorophore.

Förster theory explains FRET as a consequence of the dipole-dipole interactions between the neighboring molecules (Lakowicz, 2013) where the energy transfer efficiency (i.e. quantum yield of energy transfer transition, E) depends on the proximity of fluorophores $(r)$, it is proportional to the sixth power of the distance between donor and acceptor:

$$
E \propto \frac{1}{1+\left(\frac{r}{R}\right)^{6}}
$$

where $r$ is the distance between the two fluorophores, and $R$ is the distance at which 50\% energy transfer takes place (typically $2-7 \mathrm{~nm}$ ). $R$ reflects the properties of a particular donor/acceptor pair and depends on: quantum yield of dyes, the extent of spectral overlap between donor emission and acceptor excitation and the relative spatial orientation (flexibility) of the dipoles of the donor and acceptor fluorophores (Lakowicz, 2013).

Most importantly, FRET only functions if the emission spectrum of the donor fluorophore overlaps with the excitation spectrum of the acceptor fluorophore. Thus, an experimental design must contain two fluorophores that meet this requirement, while also minimizing the overlap between the excitation spectra of the two fluorophores. This is very important, because the most essential premise of FRET is that excitation and fluorescence of the 
acceptor fluorophore is mostly stimulated by the excited donor fluorophore being in close proximity. If both fluorophores were sufficiently excited by the initial excitation wavelength, the monitored fluorescence would not be dependent on the distance between donor and acceptor fluorophores.

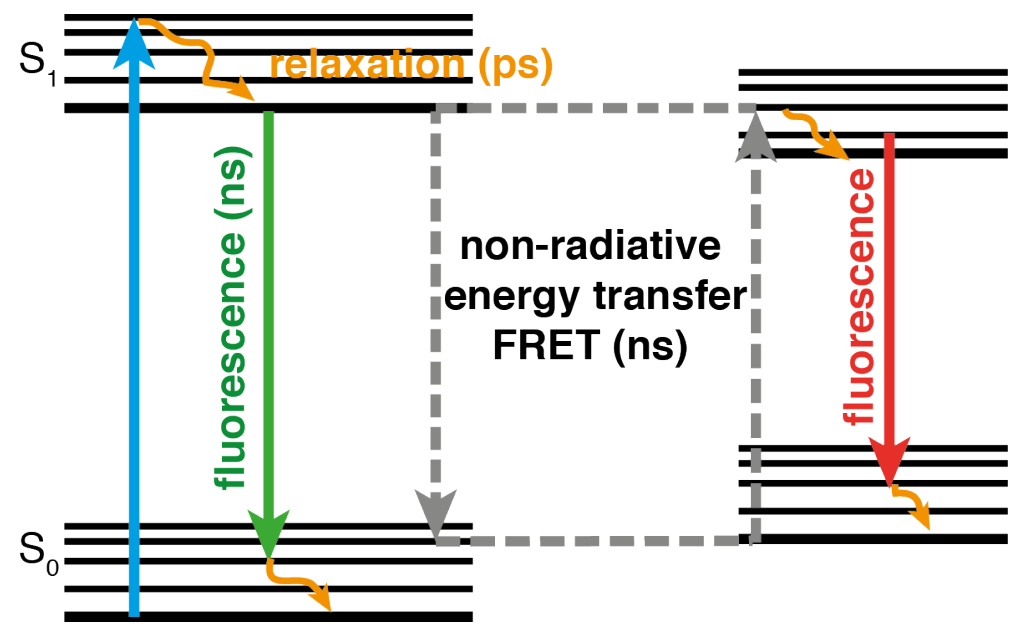

Figure 2.7. Jablonski diagram of the electronic states of donor (left) and acceptor (right) molecules in FRET. When a fluorophore absorbs light (blue arrow), electrons are excited from $S_{0}$ to $S_{1}$ state. Within a few ps, the fluorophore relaxes to the lowest vibrational level of the $S_{1}$ state (orange arrow). Subsequently, it can either relax back to the ground state emitting a photon (generating fluorescence, green arrow) or it may undergo nonradiative energy transfer (i.e. donor) to a neighboring fluorophore, thereby inducing the excitation of that fluorophore (i.e. acceptor) to $S_{1}$ state (gray dashed lines). The relaxation of the acceptor to the $S_{0}$ state generates fluorescence (red arrow). Adapted from Lakowicz, 2013. 
In this study, I used the previously established FRET-pair (Murray \& Tamm, 2009; 2011). Rhodamine Red coupled to sx TMDs (donor) and Atto647N coupled to sx-TMDs (acceptor). The protein-to-lipid ratio in my FRET measurements was 1:1,000. The reconstituted LUVs prepared with fluorescently labeled sx-TMDs were diluted 1:10 in a quartz cuvette with 25 mM HEPES buffer, containing $150 \mathrm{mM} \mathrm{NaCl}, \mathrm{pH} 7.4$ (additional components added to some mixtures are specified in the Results section). Excitation was at $560 \mathrm{~nm}$, and the emission spectra were collected from 570 to $700 \mathrm{~nm}$ with 2 nm slit widths on a FluoroMax-2 (Horiba). I corrected for cross-talk residing from acceptor excitation with samples containing only the acceptor fluorophore. The obtained FRET spectra were normalized to the maximum donor emission at $580 \mathrm{~nm}$. The FRET efficiency was calculated as the ratio of emission intensities at $660 \mathrm{~nm}$ (acceptor maximum) over $580 \mathrm{~nm}$ (donor maximum): $\mathrm{E}=\mathrm{E}_{\mathrm{AD}}{ }^{660} / \mathrm{E}_{\mathrm{AD}}{ }^{580}$. The experiments were conducted in triplicate (three independent reconstitutions). 


\section{RESULTS}

\subsection{HYDROPHOBIC MISMATCH BETWEEN THE LIPID BILAYER AND THE TRANSMEMBRANE DOMAINS DRIVES SNARE CLUSTERING}

Aiming to understand the lateral organization of plasma membrane SNAREs, I first wanted to understand if hydrophobic mismatch contributes to the clustering of syntaxin 1, a plasma membrane SNARE involved in exocytosis. Plasma membrane SNAREs syntaxin 1 and syntaxin 4 are particularly suitable to study the influence of hydrophobic mismatch on the lateral distribution of membrane proteins for two reasons.

First, both syntaxins 1 and 4 are single spanning membrane proteins which TMD lengths (21-23 AA) appear to be shorter than that needed to fully span the average hydrophobic core of the plasma membrane. This is also clear from the crystal structure of the neuronal SNARE complex (Stein et al., 2009) where the TMD segments of the SNAREs synaptobrevin 2 and syntaxin 1 seem indeed not sufficiently long to traverse the average thickness of plasma membrane of eukaryotic cells ( $\sim 4 \mathrm{~nm}$; Mitra et al., 2004). In a simulation, this resulted in defects in lipid packing, hence these SNAREs might prefer, or even organize, membrane regions of lipids with matching thicknesses. This means that in the plasma membrane syntaxin 1 transmembrane domain (sx-1 TMD) is exposed to the negative mismatch (Fig. 3.1A). In the case of negative mismatch, I speculated it would more energetically favorable to cluster syntaxins and that the membrane would adapt around the cluster generating a line tension (Fig. 3.1B).

Second, these two syntaxins segregate in separate clusters although they are homologous and structurally very similar to each other. While it was shown previously that segregation depends in part on homophilic interactions between the cytoplasmic domains (Sieber et al., 2006), it is conceivable that the small differences in the length of the TMD segments may contribute to this segregation. To isolate the effects on clustering within the membrane space from "secondary" effects caused by protein-protein interactions in the hydrophilic space, I employed truncation mutants of syntaxins 1 and 4 with their cytoplasmically oriented domains deleted. 
A

Positive mismatch Negative mismatch

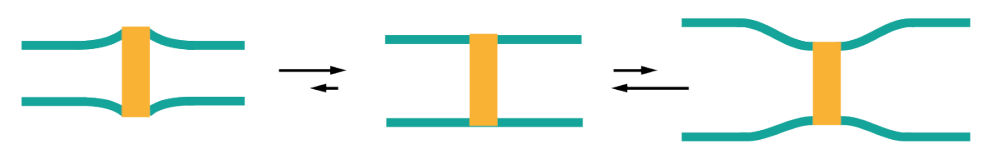

B

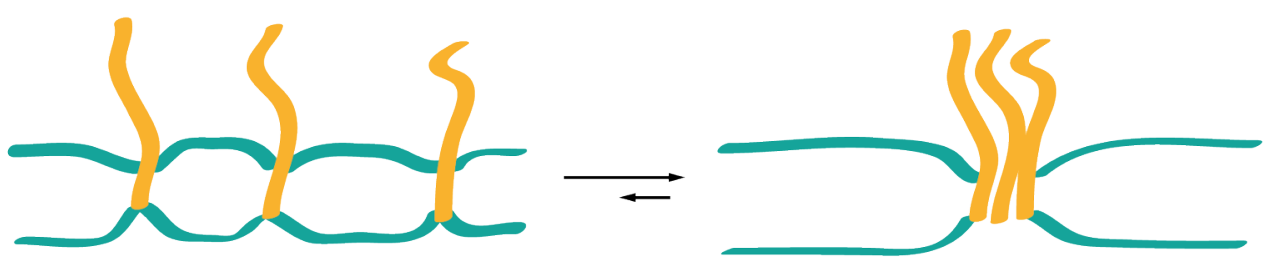

Figure 3.1. Hydrophobic mismatch between the transmembrane domain (TMD) of proteins and the surrounding lipid environment. A. The length of the TMD can match the thickness of the bilayer, be longer (positive mismatch) or shorter (negative mismatch) than the thickness of the surrounding lipid bilayer. B. It is more energetically favorable that the membrane adjusts its thickness to a cluster, rather than separately adjusting for each of the individual TMDs.

To test whether syntaxin clustering is dependent on membrane thickness, I employed an assay based on Förster resonance energy transfer (FRET) as described in Materials and Methods. More precisely, two sx-1 TMD populations labeled with spectrally separated fluorophores (RhodamineRed donor fluorophore and Atto647N - acceptor fluorophore) were mixed and incorporated into $100 \mathrm{~nm}$-sized liposomes. In case of cluster formation between sx-1 TMDs, the FRET signal would increase (Fig. 3.2A). I varied the membrane thickness by increasing the acyl chain length of PC (i.e. C14:1, C16:1, C18:1 and C20:1) and found that the FRET efficiency of sx-1 TMD was lowest (i.e. the least protein clustering) in membranes composed of C16:1 PC (Fig. 3.2B, green).

In an independent approach, I employed fluorescence correlation spectroscopy (FCS) in stacked supported lipid bilayers to assess the lateral mobility of sx-1 TMD. The lateral mobility correlates inversely with cluster formation. Indeed, I obtained a profile very similar to the FRET measurements (Fig. 3.2B, blue), with the highest mobility of sx-1 TMD in membranes of C16:1 PC. Together, these data show that syntaxin 1 clustering was lowest in membranes composed of C16:1 PC. 
A

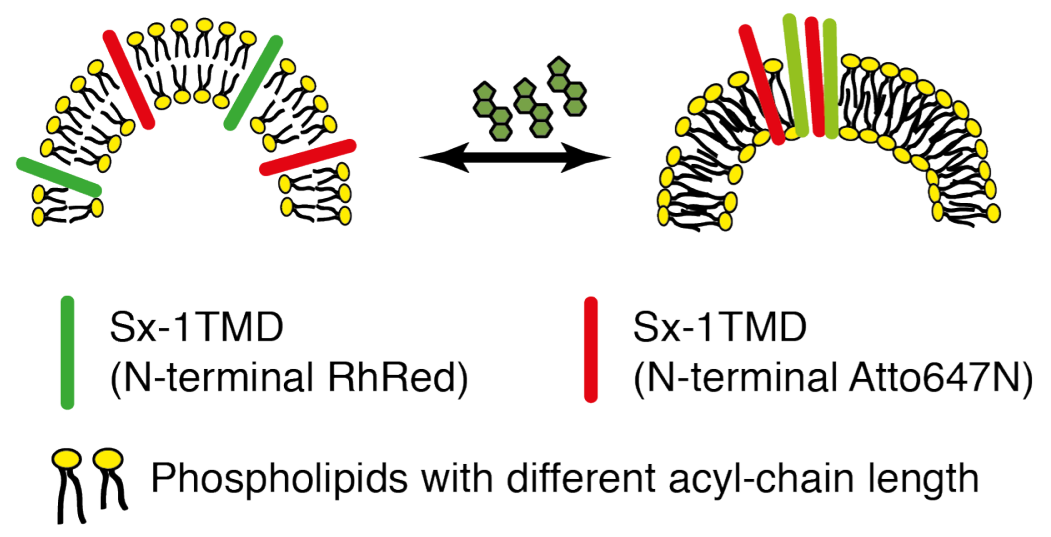

B

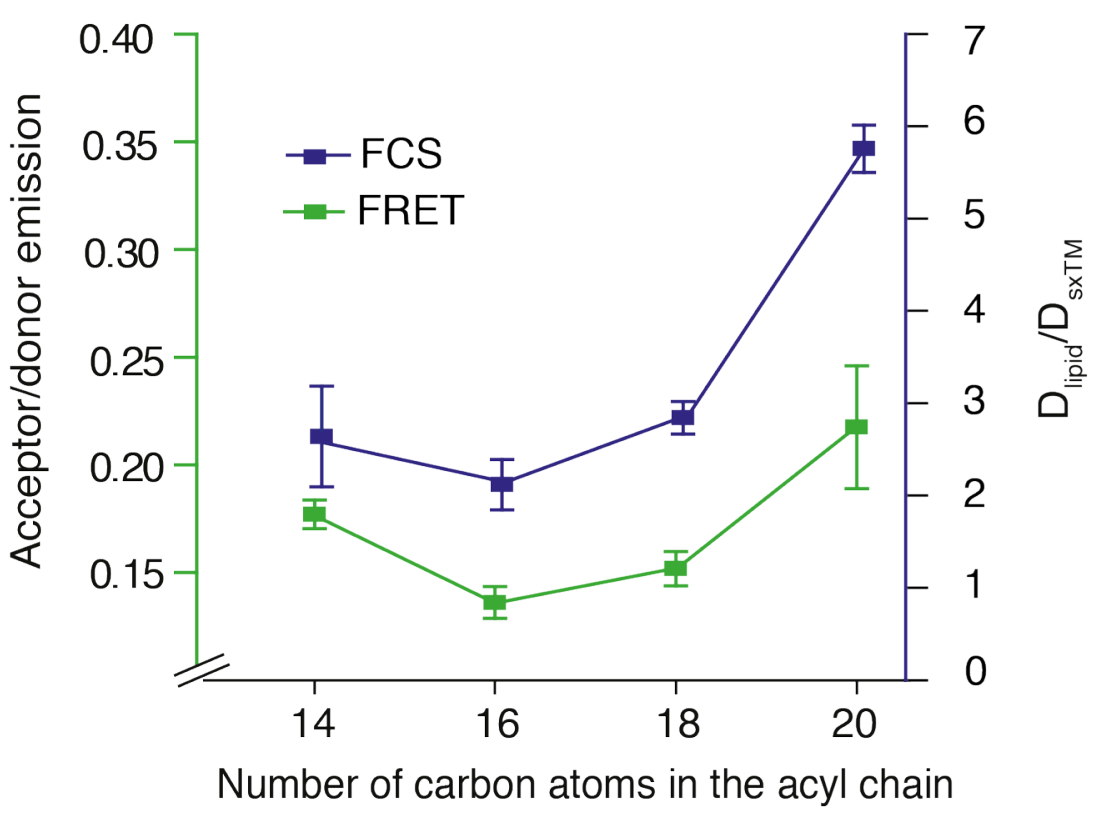

Figure 3.2. Hydrophobic mismatch clusters syntaxin 1 TMD (sx-1 TMD) in lipid bilayers. A. Scheme of FRET-based assay to measure the peptide clustering in different LUVs. Syntaxin TMDs N-terminally labeled with either Rhodamine Red or Atto647N were reconstituted in $100 \mathrm{~nm}$-sized liposomes (protein-to-lipid ratio was 1:1,000). B. Clustering determined by FRET in liposomes composed of PC of increasing acyl chain lengths (green) and FCS of sx-1 TMD labeled with Atto647N (protein/lipid ration 1:10,000) normalized to the lipid diffusion (blue). Error bars: range from two independent reconstitutions, three technical repeats each. Adapted from Milovanovic et al., 2015.

Syntaxin 4 has a 1-2 amino acids longer TMD than syntaxin 1, and this length difference is conserved in mammalian species (Introduction Fig. 1.19). Since I showed that the sx-1 TMD length determines the hydrophobic matching with the surrounding lipid environment, I expected that optimal 
matching (i.e. lowest clustering) requires a thicker bilayer for syntaxin 4 than syntaxin 1. To this end, I performed a FRET-based clustering assay using an analogous syntaxin 4 TMD peptide (sx-4 TMD), labeled at the N-terminal with the same FRET pair as sx-1 TMD (RhodamineRed and Atto647N). As shown in Fig. 3.3, the local minimum of sx-4 TMD was observed in C18:1 PC membranes. FRET data for both sx- 1 and sx-4 TMD clustering fit well with a quadratic curve $\left(a x^{2}+b x+c\right)$. In this empirical model, $-b /(2 a)$ reflects the acyl chain lengths with the lowest clustering which are 16.5 and 17.3 for sx-1TM and sx-4TM, respectively.

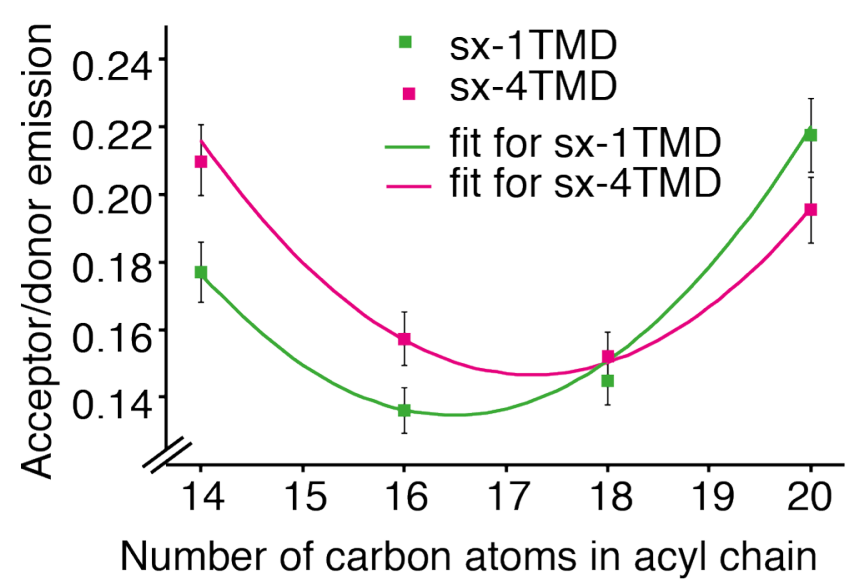

Figure 3.3. Sx-1 TMD (green) and sx-4 TMD (magenta) clustering determined by FRET assay. Sx-4 TMD with a longer transmembrane domain has a matching hydrophobic moiety shifted to bilayers composed of longer acyl chains (C18:1 PC) in contrast to syntaxin 1 (C16:1 PC). Data are fitted with quadratic curves (solid lines). Error bars: range from two independent reconstitutions, three technical repeats each. Adapted from Milovanovic et al., 2015.

Further, I employed imaging ellipsometry to determine the exact membrane thicknesses where hydrophobic matching occurs. Imaging ellipsometry assay is based on polarization changes of monochromatic light upon reflection on a bilayer (for details please see Appendix 1). Here, I determined that the bilayer thickness increases by $0.15 \mathrm{~nm}$ for each carbon unit added to the acyl chain. Based on the quadratic fit, sx- 1 TMD and sx- 4 TMD have a matching membrane thickness of $3.6 \mathrm{~nm}$ and $3.7 \mathrm{~nm}$, 
respectively. My data indicate that clustering of TMDs can be indeed driven by hydrophobic (mis)matching with the local lipid environment. Even a single amino-acid longer TMD results in a shift towards an approximately $1 \AA$ thicker membrane. Despite this difference, both syntaxins are expected to cluster since the plasma membrane has an average thickness of around $4 \mathrm{~nm}$ (Mitra et al., 2004), which would result in pronounced sequestering of these proteins to regions of decreased thickness.

The effect of cholesterol on SNARE clustering has been controversially discussed. It has been shown that SNAREs do not segregate in the Lo state induced by cholesterol, SM and saturated PC, but still the removal of cholesterol from the plasma membrane can disperse syntaxin clusters (Chamberlain et al., 2001; Lang et al., 2001; Salaün et al., 2005). Here, I wanted to address the effect of cholesterol on hydrophobic mismatch. Hence, I prepared $\sim 100 \mathrm{~nm}$ sized large unilamellar vesicles (LUVs) composed of unsaturated phosphatidylcholine (PC) with a stepwise increase of the acyl chain length (similarly as described above), either in the absence or in the presence of $30 \mathrm{~mol} \%$ cholesterol. The thickness of these membranes was determined by imaging ellipsometry measurements (Fig. 3.4A). The inclusion of $30 \mathrm{~mol} \%$ cholesterol increased the membrane thickness by approximately $0.8 \mathrm{~nm}$, independently of the acyl chain length. Next, I wanted to see if cholesterol driven membrane thickening could affect sx-1 TMD clustering. For this I used a similar FRET-based assay as described above. Indeed, in the presence of cholesterol clustering of sx-1 TMD was strongly enhanced (about $50 \%$ increase in FRET efficiency). In fact, FRET efficiencies were similar when related to membranes with the same thickness without cholesterol (Fig. 3.4B). 

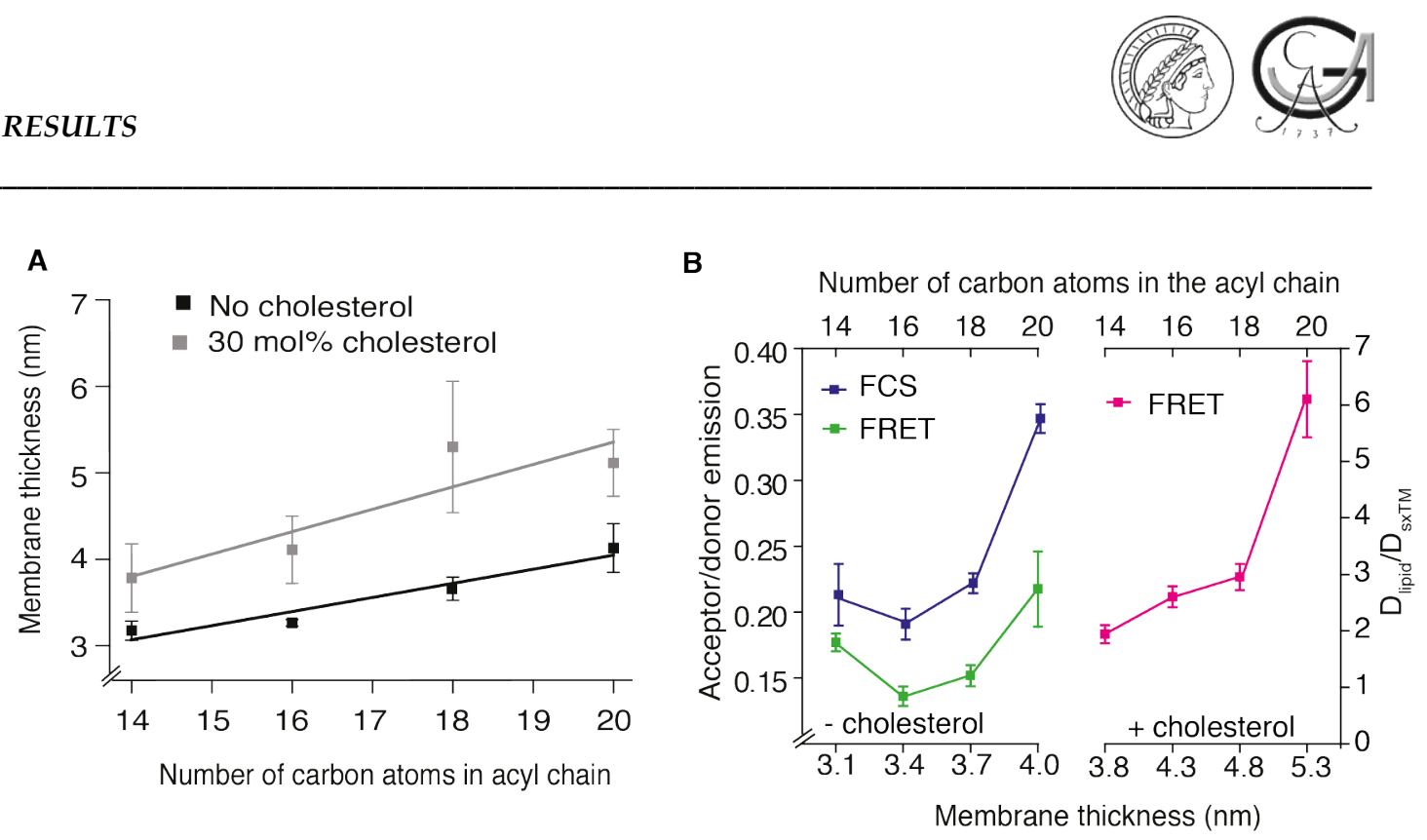

Figure 3.4. Cholesterol increases thickness of the lipid bilayer and further enhances hydrophobic mismatch. A. The thickness of supported lipid bilayers was determined by imaging ellipsometry. Lipid composition was C14:1, C16:1, C18:1 and C20:1 PC with and without $30 \mathrm{~mol} \%$ cholesterol (three independent experiments \pm SD). The linear regression analyses (solid lines) have slopes of 0.15 and 0.25 for membranes without and with cholesterol, respectively. B. Clustering determined by FRET and FCS from Fig. 3.2, but now also with membranes containing $30 \mathrm{~mol} \%$ cholesterol. Data are plotted as a function of bilayer thicknesses (bottom x-axis). Error bars: range from two independent reconstitutions, three technical repeats each. Adapted from Milovanovic et al., 2015.

To directly visualize syntaxin clustering in dependence of cholesterol, I used two imaging assays where sx- 1 TMD was reconstituted in giant unilamellar vesicles (GUVs) and polymer supported membranes (PSMs). Both GUVs and PSMs were prepared with C18:1 PC without or with $30 \mathrm{~mol} \%$ cholesterol. Here sx-1 TMD labeled with Atto647N was used to monitor its distribution and the membrane was stained with the green fluorescent lipid analogue $\mathrm{DiO}$ (3,3'-dilinoleyloxacarbocyanine). Clustering was clearly observable in the cholesterol-containing membranes and it was much less conspicuous in the absence of cholesterol (Figs. 3.5 and 3.6). This is in agreement with our FRET results described above. 

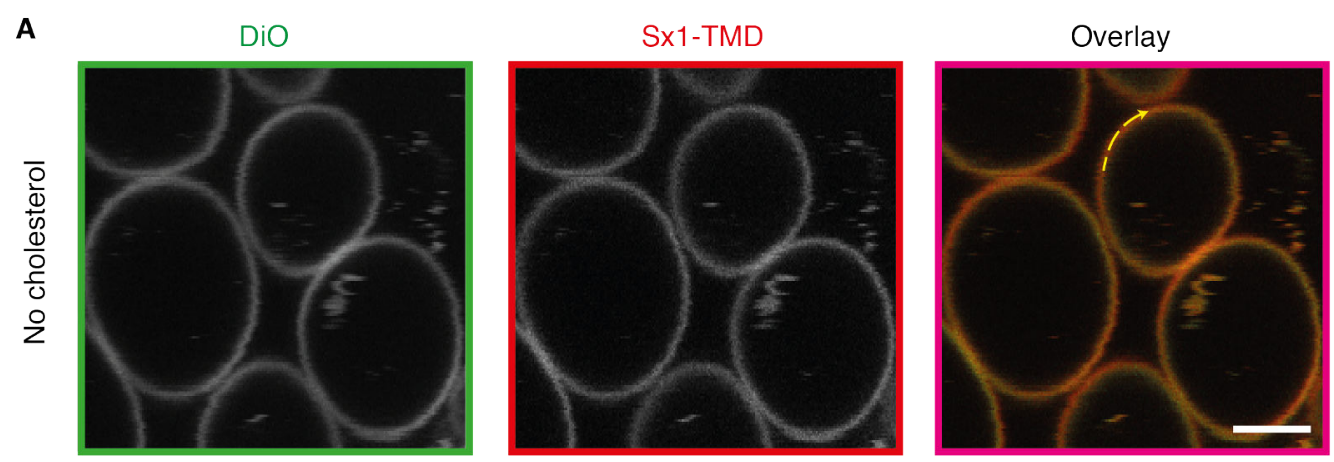

B

$\mathrm{DiO}$
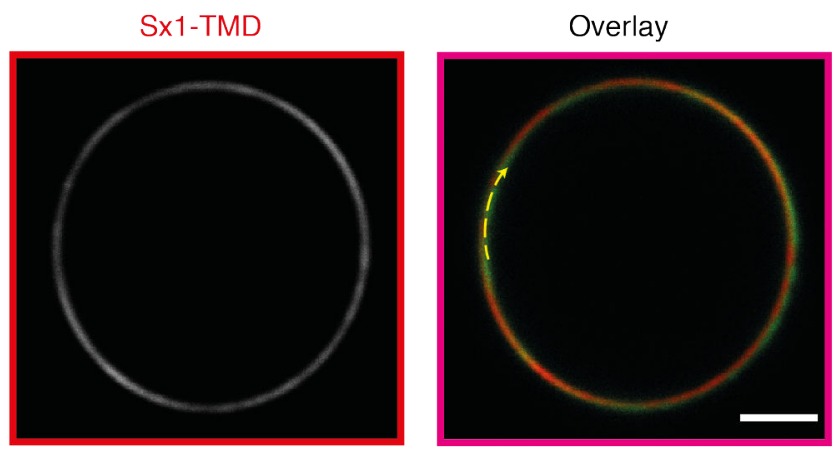

C
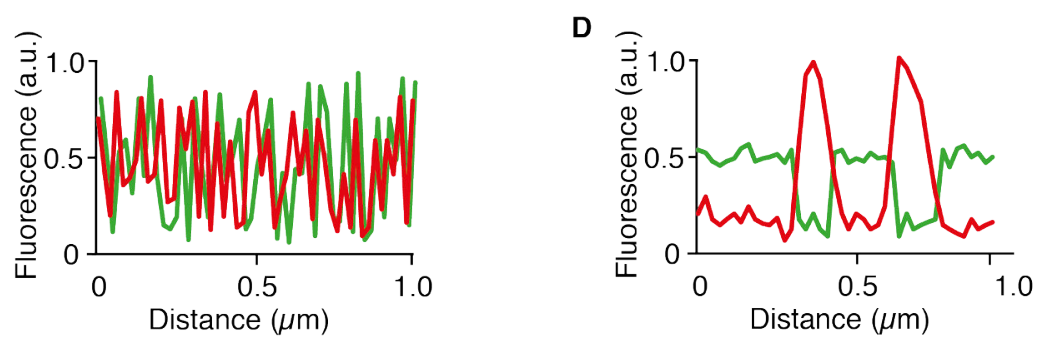

Figure 3.5. Visualizing sx-1 TMD clustering in GUVs. A. Sx-1 TMD (labeled with Atto647N, red) does not form large domains in GUVs composed of C18:1 PC. B. Cholesterol induces sx-1 TMD clustering in GUVs composed of C18:1 PC and $30 \mathrm{~mol} \%$ cholesterol. Protein-to-lipid ratio was 1:1,000. DiO (0.01 $\mathrm{mol} \%$ ) was used as lipophilic membrane marker (green). C, D. Fluorescence intensity along yellow lines in $A$ and $B$, respectively. Scale bars, $1 \mu \mathrm{m}$.

From both FRET assays and membrane reconstitution experiments, I clearly see that syntaxin clustering is not due to cholesterol-induced phase separation of membrane lipids since none of the membrane systems contained lipids with saturated fatty acids required for the partitioning into Lo and Ld phases. Rather, my data demonstrate that the effect of cholesterol increases the membrane thickness, which results in syntaxin clustering due to increased hydrophobic mismatch. 
A

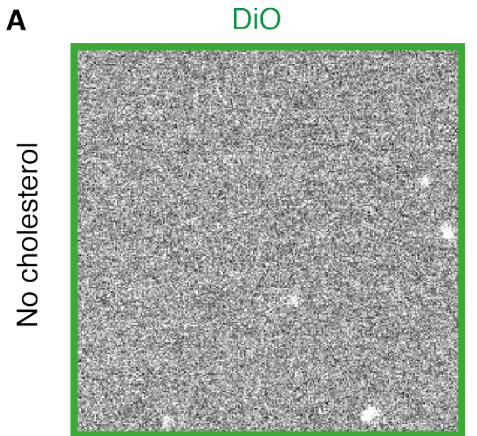

B

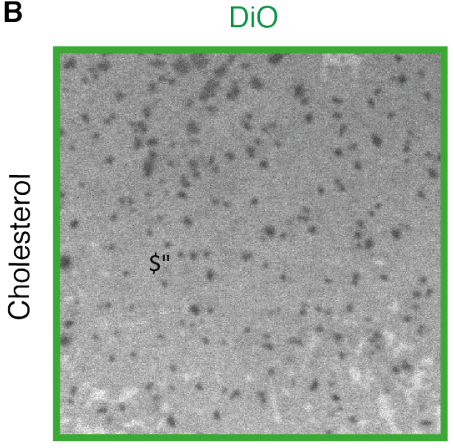

C

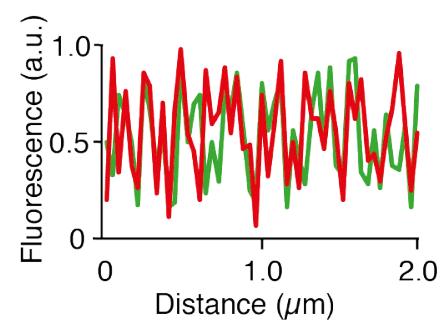

Sx1-TMD

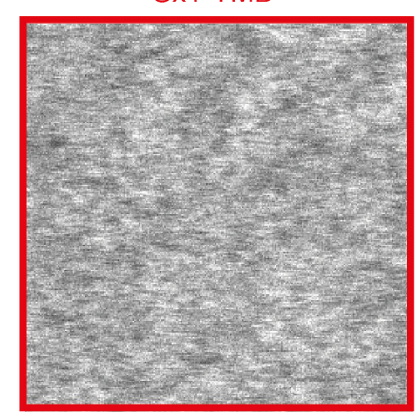

Sx1-TMD

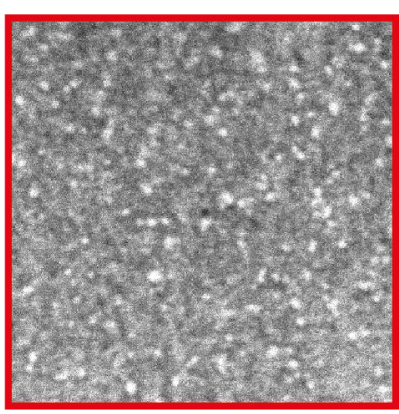

D

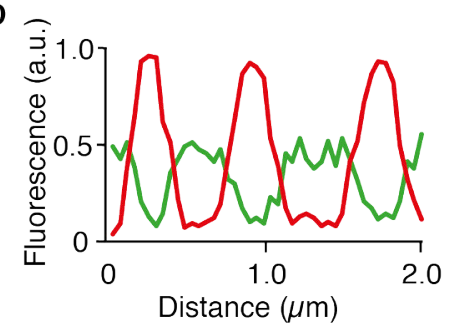

Figure 3.6. Reconstitution of sx-1 TMD in polymer supported membranes. A. Sx-1 TMD (Atto647N, red) does not form domains in C18:1 PC membranes. B. The mixture of C18:1 PC and $30 \mathrm{~mol} \%$ cholesterol induces sx-1 TMD domain formation. Protein-to-lipid ration was 1:10,000. $\mathrm{DiO}(0.01 \mathrm{~mol} \%$, green) was used as lipophilic membrane marker. C, D. Fluorescence intensity along yellow lines in $A$ and $B$, respectively. Scale bars, $2 \mu \mathrm{m}$. Adapted from Milovanovic et al., 2015.

To further understand the interaction between sx-1 TMD and the lipid bilayer, I characterized the influence of homotypic TMD interactions on clustering. Previously, it has been shown that syntaxin 1 TMDs homodimerize in membranes. This homodimerization depends on specific protein-protein interactions in the hydrophobic phase that can be disrupted by alanine 
substitutions of three hydrophobic side chains within the TMD (M267A, C271A, I279A) (Fig. 3.7A; Laage, Rohde, Brosig, \& Langosch, 2000). To examine if this homodimerization contributes to homophilic clustering during hydrophobic mismatch, I reconstituted the corresponding sx-1 TMD mutant peptides and measured clustering by FRET.

A

Syntaxin $1 \mathrm{~A}$

$\mathrm{N}$-terminal regulatory SNARE-motif TMD

Homo sapiens
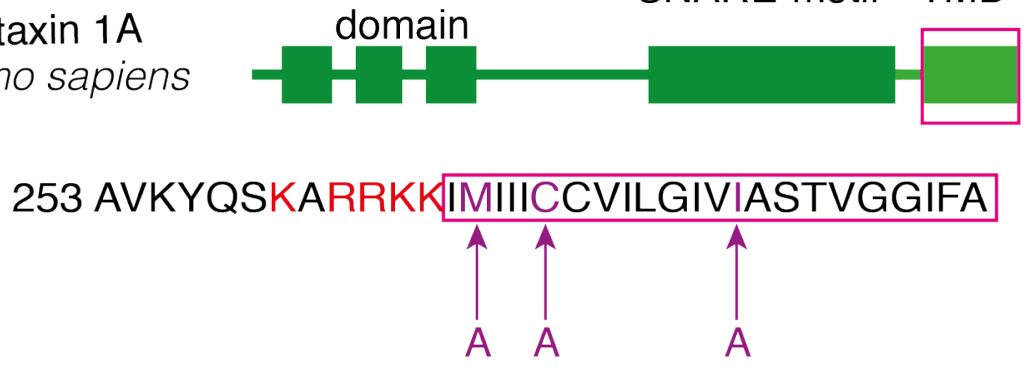

B

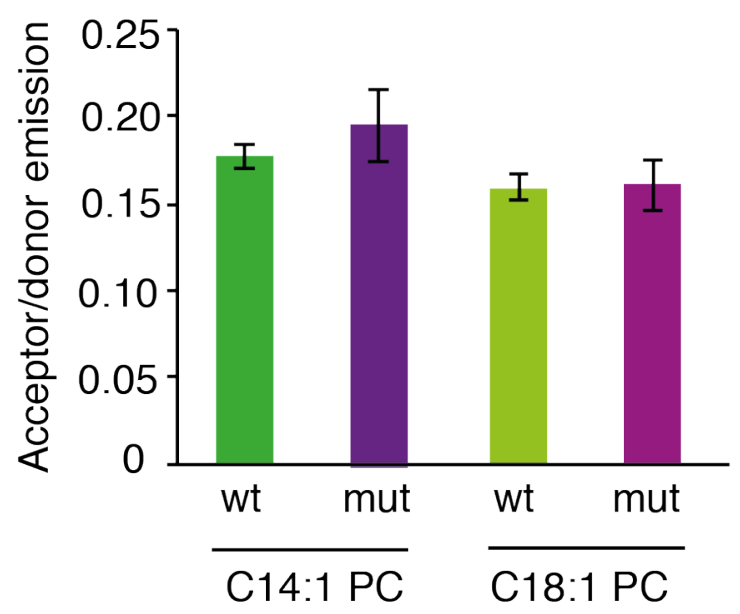

Figure 3.7. Specific protein-protein interactions of TMDs do not affect clustering in case of positive and negative mismatch. A. Scheme of syntaxin 1 domain organization with the part of the sequence (residues 253 to 288). Mutated residues within TMD are marked in purple. B. Comparison of sx-1 TMD clustering between wild-type and mutant sequence in liposomes composed of C14:1 and C18:1 PC. Error bars: range from two independent reconstitutions, three technical repeats each. Adapted from Milovanovic et al., 2015.

In membranes composed of C14:1 PC and C18:1 lipids (i.e. both thinner and thicker than required for optimal hydrophobic matching of syntaxin 1), the dimerization mutant clustered comparably to the wild-type (Fig. 3.7B), indicating that at these conditions homotypic interactions of the TMDs were not required for clustering. These results demonstrate that even if there is a 
specific protein-protein interaction at the TMD region, hydrophobic mismatch can further enhance the clustering. This is especially important given that the plasma membrane is a system with pronounced negative mismatch environment.

\subsection{HYDROPHOBIC MISMATCH SHAPES SYNTAXIN CLUSTERS TOGETHER WITH THE SURFACE IONIC INTERACTIONS AND SPECIFIC PROTEIN-PROTEIN INTERACTIONS}

Syntaxin 1 contains a polybasic motif juxtaposed to its TMD (shown in red, Fig. 3.7A) that is known to interact with $\mathrm{PI}(4,5) \mathrm{P}_{2}$ and/or $\mathrm{PI}(3,4,5) \mathrm{P}_{3}$ (Aoyagi et al., 2005; Khuong et al., 2013; Murray \& Tamm, 2009; van den Bogaart et al., 2011). Both phosphoinositides are highly accumulated in at least a fraction of syntaxin 1 clusters in the plasma membrane (Aoyagi et al., 2005; James et al., 2008; Murray \& Tamm, 2011; van den Bogaart et al., 2011).

Two-color super-resolution STED microscopy imaging of neuroendocrine PC12 plasma membrane sheets confirmed the enrichment of $\mathrm{PI}(4,5) \mathrm{P}_{2}$ in syntaxin 1 clusters (Fig. 3.8A). Syntaxin 4 also contains a similar polybasic motif and $\mathrm{PI}(4,5) \mathrm{P}_{2}$ was also enriched in syntaxin 4 clusters (Fig. $3.8 \mathrm{~B})$. The overall density of $\mathrm{PI}(4,5) \mathrm{P}_{2}$ clusters $\left(13.9 \pm 1.6\right.$ clusters $\left./ \mu \mathrm{m}^{2}\right)$ was 3 times higher than the cluster density of syntaxin $1\left(4.5 \pm 0.4\right.$ clusters $\left./ \mu \mathrm{m}^{2}\right)$ and syntaxin $4\left(5.4 \pm 0.7\right.$ clusters $\left./ \mu \mathrm{m}^{2}\right)$. This is not surprising considering that $\mathrm{PI}(4,5) \mathrm{P}_{2}$ interacts with many other proteins in cells (Di Paolo \& De Camilli, 2006; McLaughlin et al., 2002). 


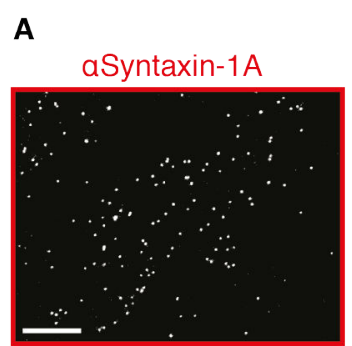

B

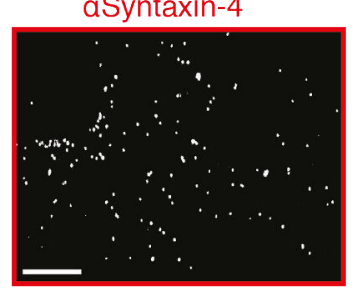

$\mathrm{aPI}(4,5) \mathrm{P}_{2}$

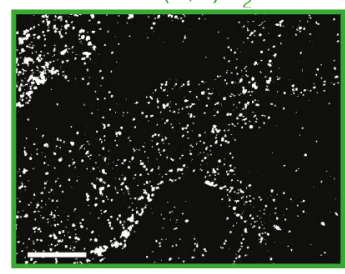

$\operatorname{aPl}(4,5) P_{2}$

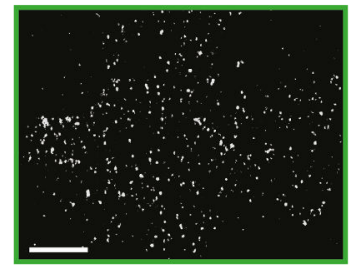

Overlay
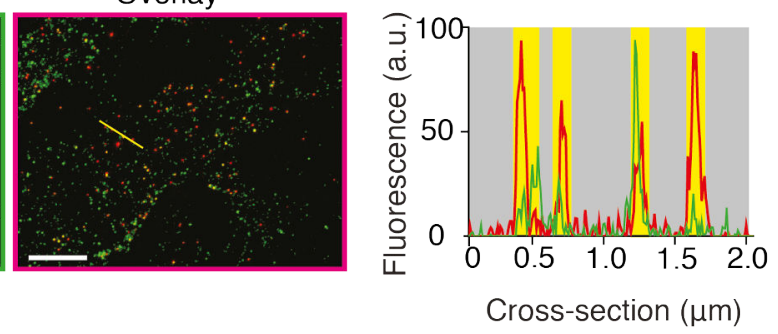

Overlay

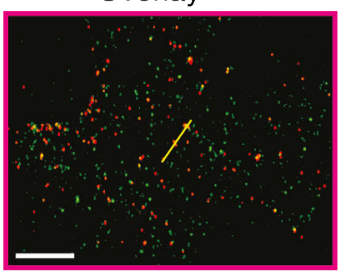

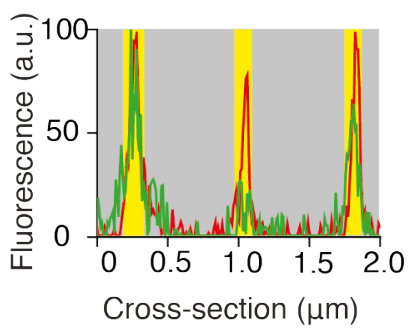

Figure 3.8. Two color STED images of syntaxin 1 and 4 colocalization with the $\mathrm{PI}(4,5) \mathrm{P}_{2}$ in the plasma membrane sheets of PC12 cells. The plasma membrane sheets were immunostained for $\mathrm{PI}(4,5) \mathrm{P}_{2}$ (green) and either syntaxin 1 (A, red) and syntaxin 4 (B, red). The graphs represent fluorescence line profiles as indicated in the images. Yellow highlights show the position of the domains. Scare bars, $2 \mu \mathrm{m}$. Adapted from Milovanovic et al., 2015.

Next I wanted to see if $\mathrm{PI}(4,5) \mathrm{P}_{2}$ increases co-clustering of syntaxins 1 and 4. Hence, I measured FRET between sx-1 TMD labeled with Rhodamine Red (donor fluorophore) and sx-4 TMD labeled with Atto647N (acceptor fluorophore) in LUVs (Fig. 3.9A). Indeed, the presence of $1 \mathrm{~mol} \% \mathrm{PI}(4,5) \mathrm{P}_{2}$ in LUVs composed of brain PC caused an increase of the FRET efficiency, indicating that the TMDs of the two syntaxin isoforms co-clustered in the membrane when $\mathrm{PI}(4,5) \mathrm{P}_{2}$ was present. To check if electrostatic interactions at the surface are not abolished when cholesterol is present (i.e. pronounced negative hydrophobic mismatch), I measured FRET in the presence of both $\mathrm{PI}(4,5) \mathrm{P}_{2}$ and cholesterol (Fig. 3.9B). The clustering was present in the mixture that contained both $\mathrm{PI}(4,5) \mathrm{P}_{2}$ and cholesterol indicating that electrostatic interactions corroborate with hydrophobic mismatch. 
A

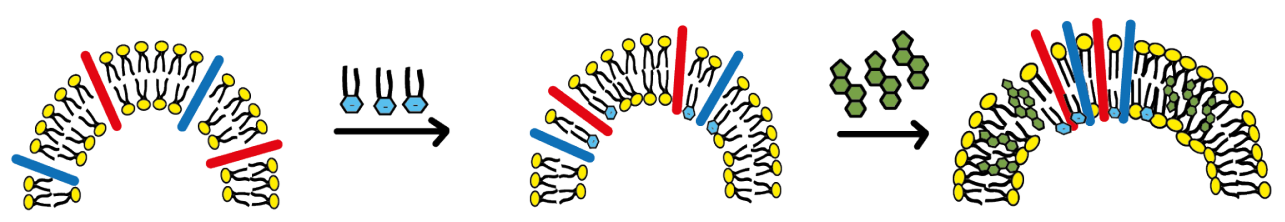

sx-1 TMD

(N-terminal RhRed)

(f) $\mathrm{PI}(4,5) \mathrm{P}_{2}$

8 Cholesterol

sx-4 TMD

(N-terminal Atto647N)

R Phospholipids

B

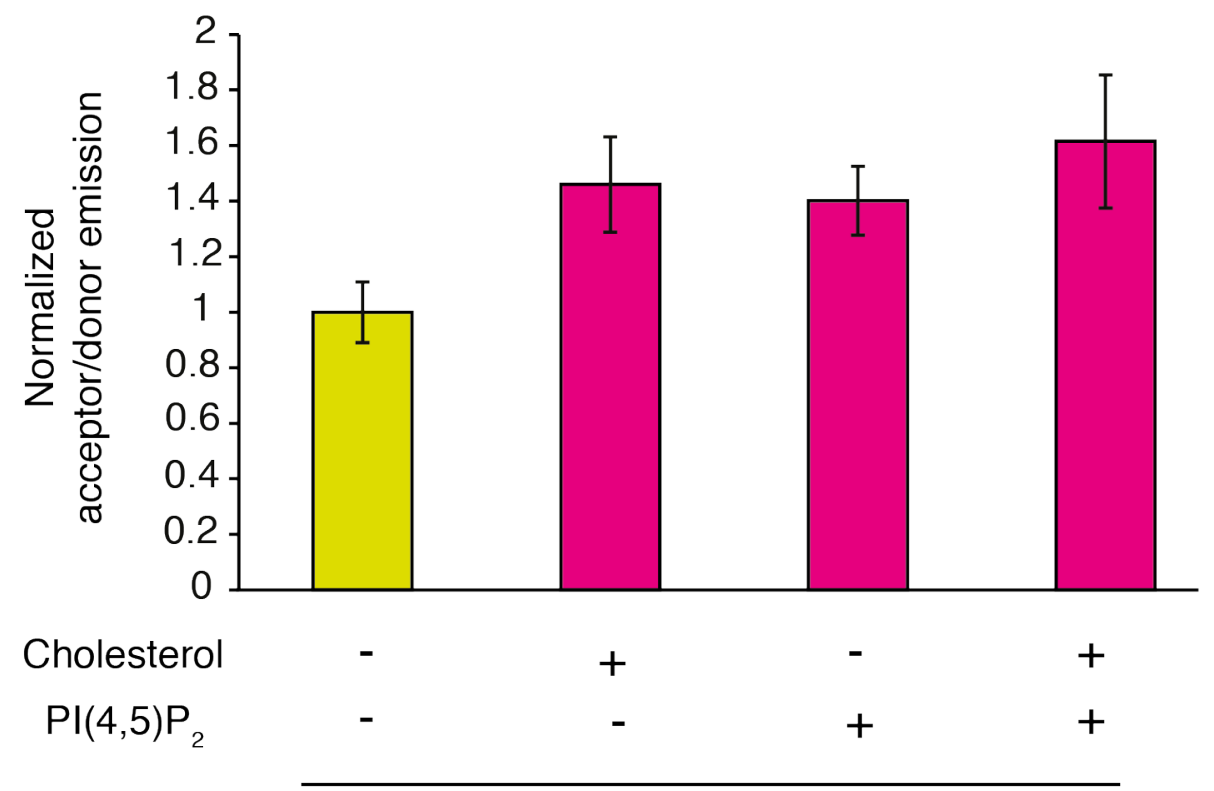

Brain PC

Figure 3.9. Cholesterol and $\mathrm{PI}(4,5) \mathrm{P}_{2}$ both induce clustering of syntaxin TMDs. A. Scheme of the FRET-based clustering assay with $\mathrm{PI}(4,5) \mathrm{P}_{2}$ and cholesterol incorporated in $100 \mathrm{~nm}$ liposomes. Sx-1 and sx-4 TMDs are labeled with Rhodamine Red and Atto647N, respectively. B. Sx-1 and sx-4 TMDs coclustering is enhanced by both $\mathrm{PI}(4,5) \mathrm{P}_{2}$ and cholesterol. FRET was measured in LUVs composed of porcine brain PC without or with $1 \mathrm{~mol} \%$ $\mathrm{PI}(4,5) \mathrm{P}_{2}$, and/or $30 \mathrm{~mol} \%$ cholesterol ( \pm range from two independent reconstitutions, three technical repeats each). Adapted from Milovanovic et al., 2015. 
In order to dissect how the electrostatic repulsion between the polybasic linker regions affects clustering by hydrophobic mismatch, I repeated the FRET assay in the presence of high concentrations of $\mathrm{NaCl}$. High concentrations of ions could counterbalance the charged amino acids. Indeed, membrane clustering of sx-1 TMD was enhanced when electrostatic interactions were screened (buffer with $1 \mathrm{M} \mathrm{NaCl}$ ). This finding indicates that the repulsion of the polybasic linkers of syntaxins under physiological ionic strength (i.e. $150 \mathrm{mM}$ ) can counteract hydrophobic mismatch and partially limit clustering even in the presence of $\mathrm{PI}(4,5) \mathrm{P}_{2}$ (Fig. 3.10).

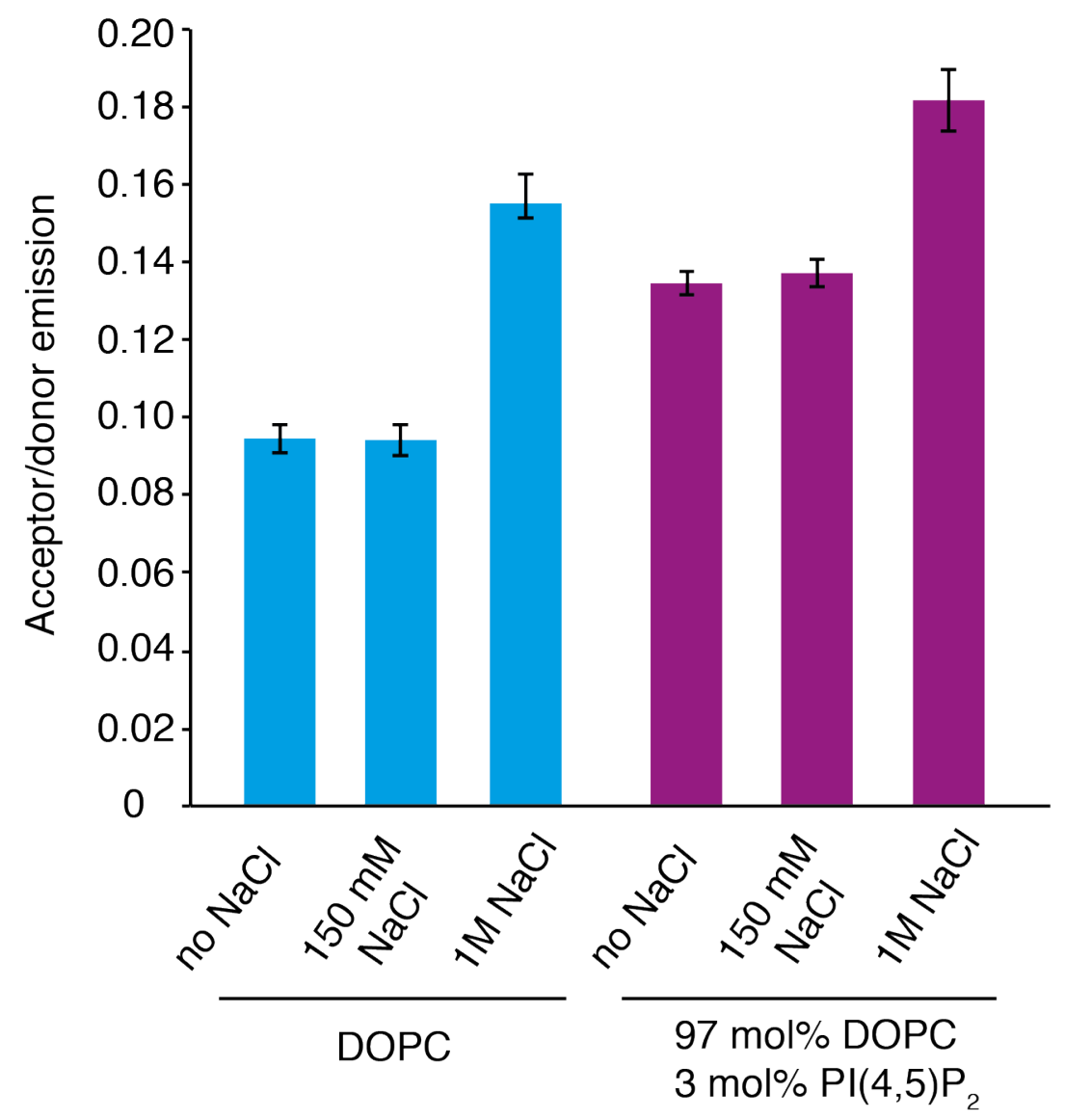

Figure 3.10. Determining the charge screening effect on sx-1TMD and $\mathrm{PI}(4,5) \mathrm{P}_{2}$ association by FRET assay. Clustering of sx-1 TMD was measured by FRET in the presence or absence of $150 \mathrm{mM}$ or $1 \mathrm{M} \mathrm{NaCl}$; and in DOPC liposomes without or with $3 \mathrm{~mol} \% \mathrm{PI}(4,5) \mathrm{P}_{2}$. Error bars: range from two independent reconstitutions, three technical repeats each. Adapted from Milovanovic et al., 2015. 
Further, I analyzed whether the varying thicknesses of the membrane bilayer could at least partially segregate syntaxins 1 and 4 considering the mismatch in length between their TMDs. It is well known that syntaxin 1 and 4 segregate into distinct domains in the plasma membrane (Fig. 3.11) and this has been largely contributed to homophilic interactions between SNARE motifs (Barg et al., 2010; Sieber et al., 2006; 2007). To address if the TMDs contribute to this conspicuous segregation, I first reconstituted sx-1 TMD and sx-4 TMD in liposomes composed of a mixture of PC with different acyl chain lengths (C14:1 to C20:1) and measured clustering by FRET. Clustering of sx-1 TMD with sx-4 TMD, but not of sx-1 TMD to sx-1 TMD and sx-4 TMD to sx-4 TMD, was lower compared to liposomes containing only C18:1 PC (Fig. 3.12). This demonstrates that syntaxin TMDs preferentially clusters in regions containing lipids with matching hydrophobic thickness.
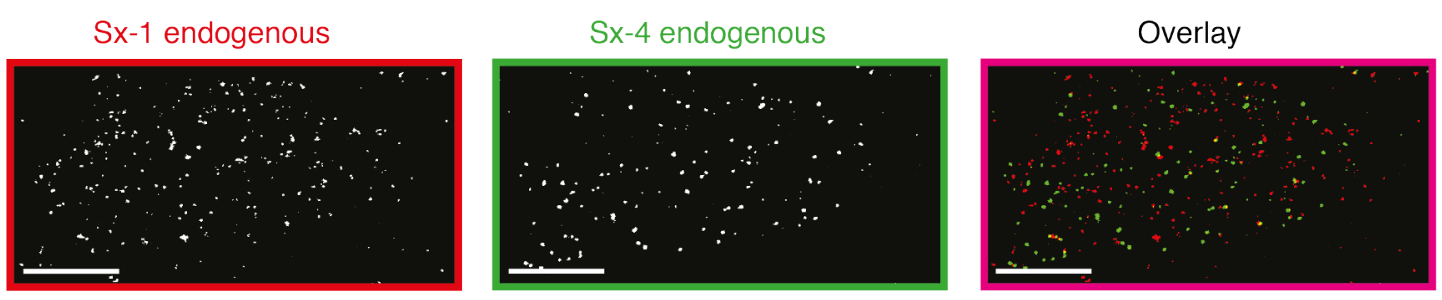

Figure 3.11. Two-color STED microscopy of PC12 membrane sheets immunostained against syntaxins 1 (red) and 4 (green). Endogenous syntaxins 1 and 4 segregate into distinct membrane domains in the complex plasma membrane environment. Scale bars $2 \mu \mathrm{m}$. Adapted from Milovanovic et al., 2015. 


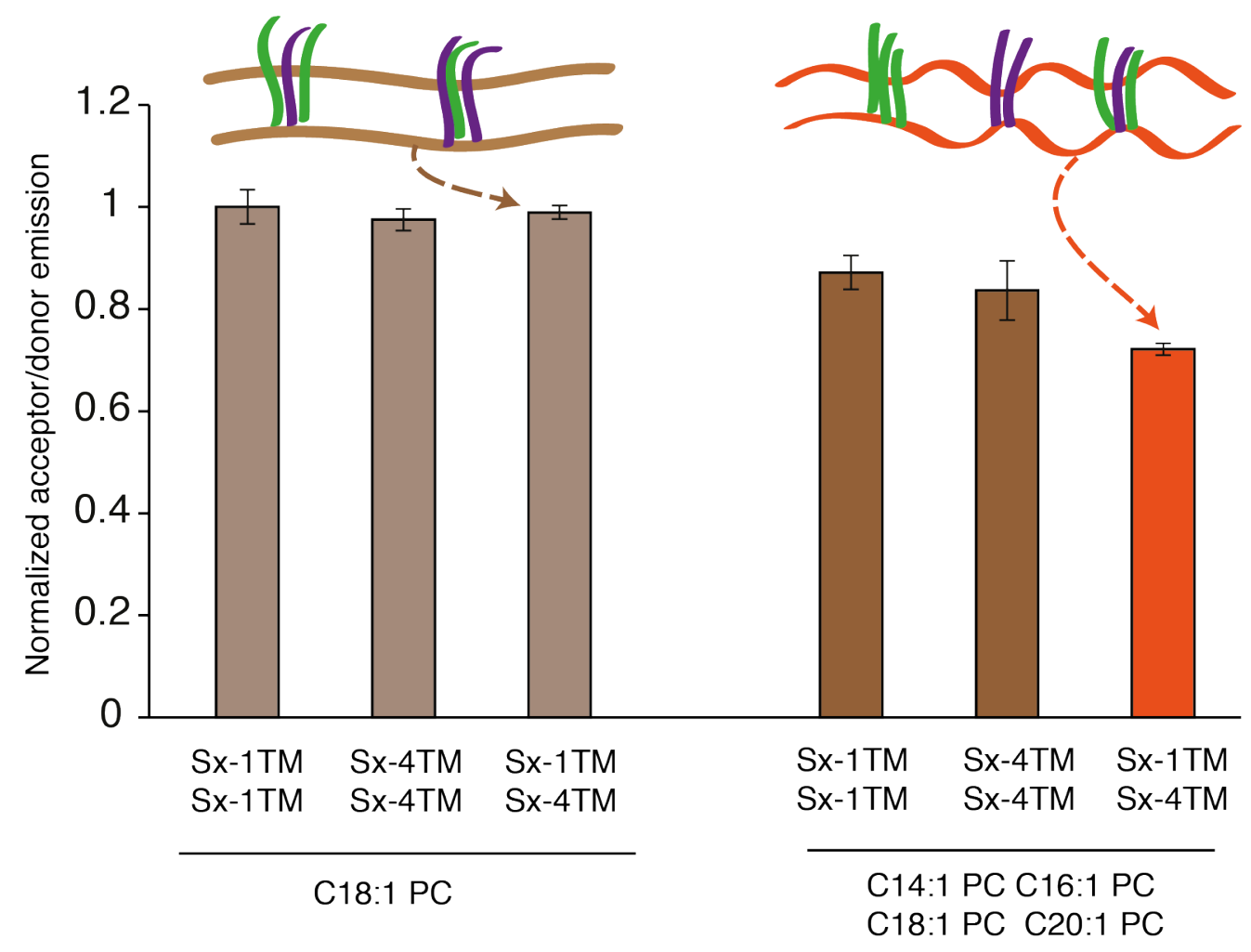

Figure 3.12. Clustering of sx-1 TMD and sx-4 TMD measured by FRET assay in liposomes composed of either C18:1 PC (left; top: scheme, bottom: data) or an equimolar mixture of C14:1, C16:1, C18:1 and C20:1 PC (right; top: scheme, bottom: data). All liposomes contained $1 \mathrm{~mol} \% \mathrm{PI}(4,5) \mathrm{P}_{2}$. Reduced clustering of TMDs was observed in liposomes that contained a mixture of acyl chains with different lengths (i.e. varying thickness). Error bars: range from two independent reconstitutions, three technical repeats each. Adapted from (Milovanovic et al., 2015).

To address if the TMDs of syntaxins 1 and 4 would also contribute to their segregation in the complex plasma membrane environment, I transfected PC12 cells with truncation mutants of both syntaxins (sx-1TM and sx-4TM, similar to the fragments used in abovementioned experiments). These syntaxin 1 and 4 mutants were N-terminally fused to GFP and mCherry, respectively. When membrane sheets from these cells were analyzed by two-color superresolution STED microscopy, segregation of the two mutants in separate clusters was observed (Fig. 3.13A). PC12 membrane sheets were fixed and immunostained against GFP and mCherry tags as 
described in Material and Methods. Expression of constructs coding for either full-length syntaxins as well as for only the TMDs showed similar clustering (Fig. 3.13B). It has been shown previously that sx-1TM does not colocalize with the full-length syntaxin 1 (Sieber et al., 2007).

A

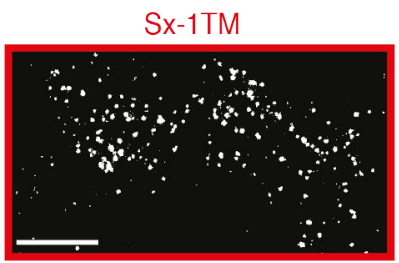

Sx-1TM
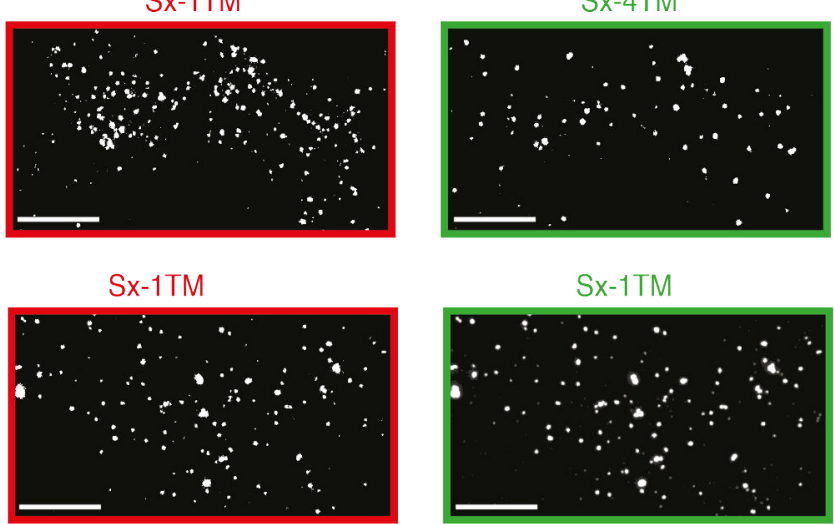

Sx-1TM
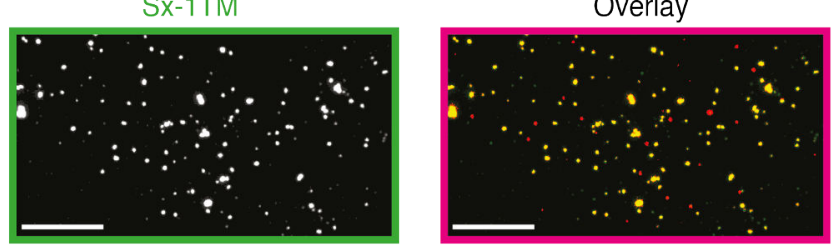

B

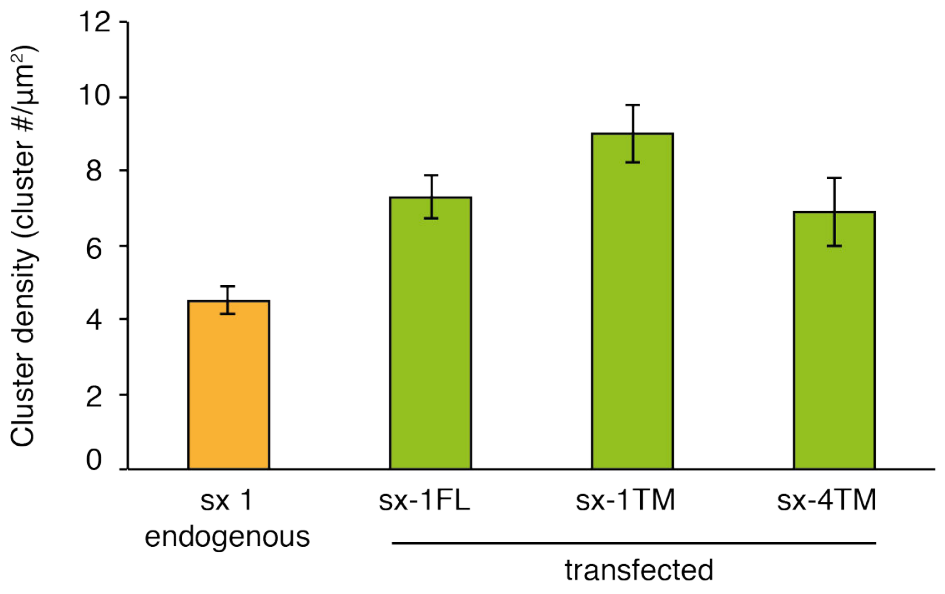

C

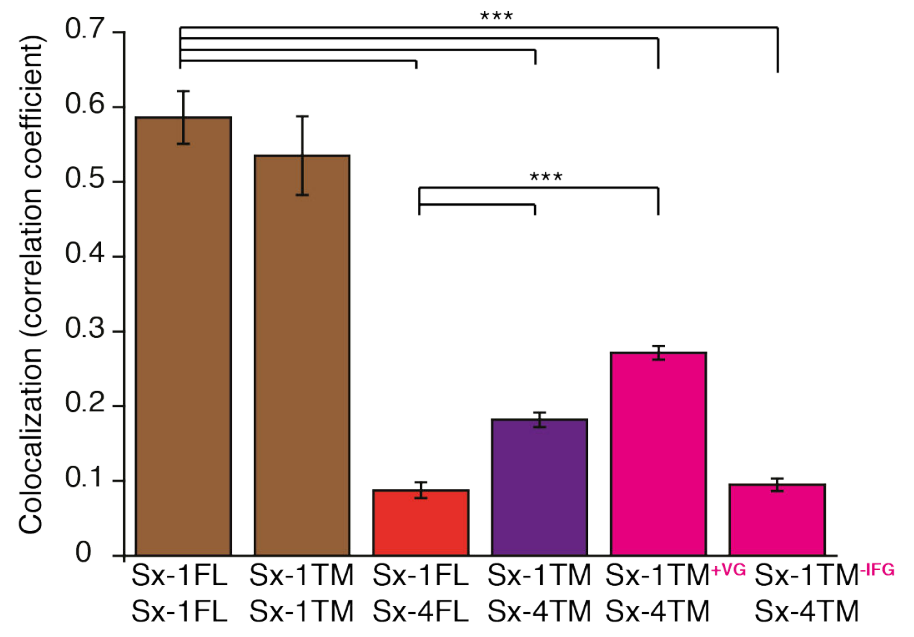


Figure 3.13. Differences in the length of the TMDs contribute to segregation of syntaxin 1 and 4 into distinct domains in the plasma membrane. A. Top: Twocolor STED images of PC12 cell sheets expressing sx-1TM (fused with mCherry, red) and sx-4TM (fused with EGFP, green). Bottom: Two-color STED images of PC12 membrane sheets expressing sx-1TM fused to mCherry (red) and EGFP (green) as a control experiment with high colocalization coefficients. B. Comparison of average cluster density between endogenously expressing syntaxin 1 and transfected with syntaxin 1 full length (sx-1 FL), sx1TM and sx-4TM. C. Pearson correlation coefficient shows the extent of overlap of clusters in PC12 membrane sheets transfected with various syntaxin TMD mutants (sx-1 FL and sx-4 FL; full length constructs of syntaxin 1 and syntaxin 4 respectively); Each analysis included at least 10 sheets from three independent transfections $\left({ }^{* *} \mathrm{p}<0.001\right.$, two-sided, unpaireded t-test, error bars show s.e.m). Scale bar, $2 \mu \mathrm{m}$. Adapted from Milovanovic et al., 2015.

To test the hypothesis that syntaxin 1 and syntaxin 4 segregation into distinct domains was due to the difference in length of the TMDs, I generated syntaxin 1 TMDs that were either two amino acids longer $\left(s x-1 T M^{+V G}\right)$ or three amino acids shorter (sx-1TM-IFG) than the wild type. In line with the hydrophobic mismatch hypothesis, clusters of the shorter sx-1TM-IFG strongly segregated from sx4-TM clusters (Fig. 3.13C). In contrast, the longer sx$1 \mathrm{TM}+\mathrm{VG}$, with a similar length of TMD as sx-4 TMD, showed significantly more co-localization with sx-4 TMD. Overall, data presented here show that increasing or reducing hydrophobic mismatch by altering the length of TMDs by only a few residues contributes to both the extent of protein clustering as well as to the segregation into separate clusters. 


\subsection{CALCIUM ACTS AS A CHARGE BRIDGE THAT CONNECTS MULTIPLE SYNTAXIN 1/PI(4,5) $P_{2}$ CLUSTERS INTO LARGER MESOSCALE DOMAINS}

In the final part of the project I addressed the surface chelating effect of multivalent cations such as $\mathrm{Ca}^{2+}$ on syntaxin clustering. Previously $\mathrm{Ca}^{2+}$ was shown to increase clustering of SNAREs in the plasma membrane including syntaxin 1 (Zilly et al., 2011). This effect was explained by the overall negative surface charge (i.e. sum of all lysines and arginines minus sum of glutamates and aspartates) of plasma membrane SNAREs. Here, $\mathrm{Ca}^{2+}$ would chelate the carboxyl groups of the side chains at the protein surface. However, $\mathrm{Ca}^{2+} \mathrm{can}$ also enhance clustering of syntaxin 1 that contains a series of polybasic residues juxtaposed to the TMD, just at the membrane surface (total charge of $+5)$. I first confirmed the previously reported finding that elevated $\mathrm{Ca}^{2+}$ promotes clustering of syntaxin 1 in the plasma membrane. Using STED microscopy I obtained high-resolution images of PC12 plasma membranes immunolabeled for syntaxin 1 (Fig. 3.14A). After analyzing at least ten cell sheets from three different experiments, I observed that the average cluster density of syntaxin 1 increased from $3.3 \pm 0.3$ clusters $/ \mu \mathrm{m}^{2}$ to $4.1 \pm 0.4$ clusters $/ \mu \mathrm{m}^{2}$ upon the addition of $150 \mu \mathrm{M} \mathrm{Ca}^{2+}$ (Fig. 3.14B).

Apart from the increase in density, $\mathrm{Ca}^{2+}$ shifts the size of domains to the slightly larger values (average domain diameter was $\sim 90 \mathrm{~nm}$ and $\sim 105 \mathrm{~nm}$ before and after $\mathrm{Ca}^{2+}$, respectively; Fig. 3.14C). Of course, these are upper estimates of domain sizes since these experiments are based on antibody staining, and so-called umbrella effect may result in a larger apparent domain sizes (Sieber et al., 2007). 
A Syntaxin 1/Atto647N

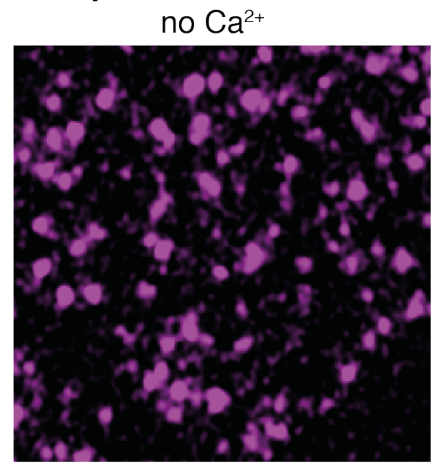

B

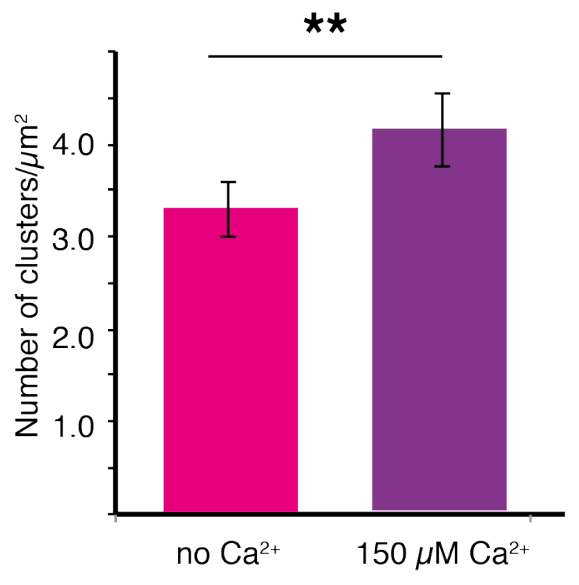

Syntaxin 1/Atto647N $150 \mu \mathrm{M} \mathrm{Ca}^{2+}$

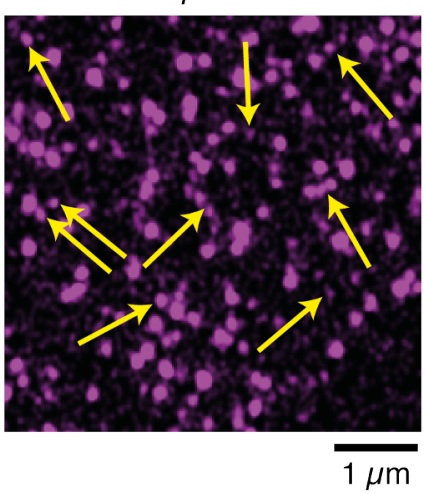

C

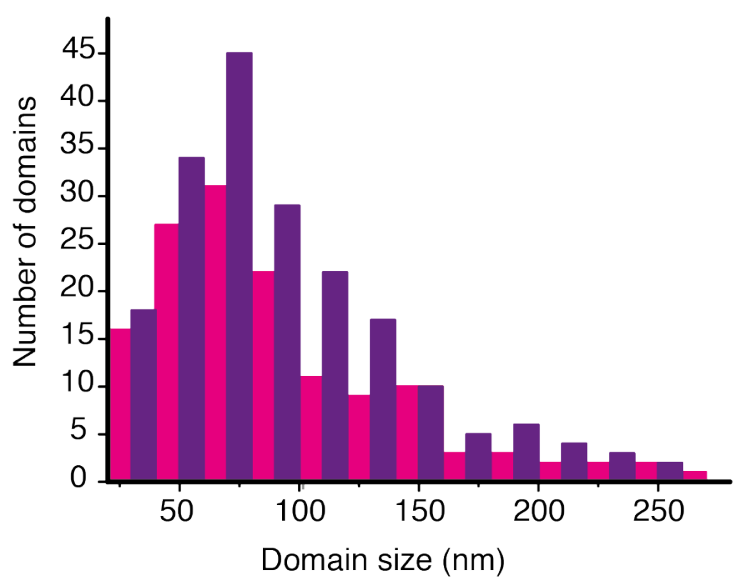

Figure 3.14. Calcium promotes clustering of syntaxin 1 in the plasma membrane of PC12 cells. A. STED images of the plasma membrane immunostained against syntaxin 1 before (left) and after (right) addition of $150 \mu \mathrm{M} \mathrm{Ca}^{2+}$. Scale bar $1 \mu \mathrm{m}$. Note the appearing of new syntaxin domains upon addition of $\mathrm{Ca}^{2+}$. B. Density of syntaxin 1 clusters increases $\sim 25 \%$ after addition of $\mathrm{Ca}^{2+}$. Error bars: range from three independent experiments with at least ten sheets analyzed. ( ${ }^{* *} P<0.01$, two-sided, unpaired $t$-test). C. Size of syntaxin 1 domains in the plasma membrane without (pink) and with (purple) $150 \mu \mathrm{M} \mathrm{Ca}^{2+}$. 


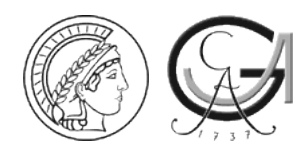

A

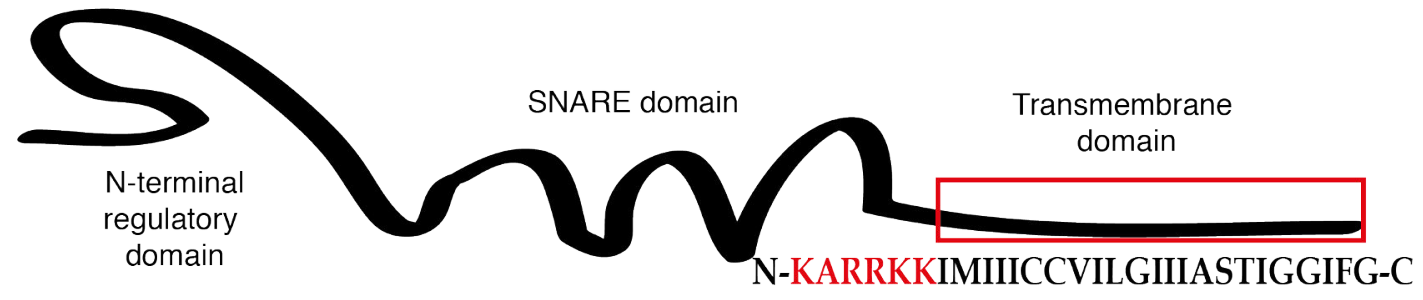

B

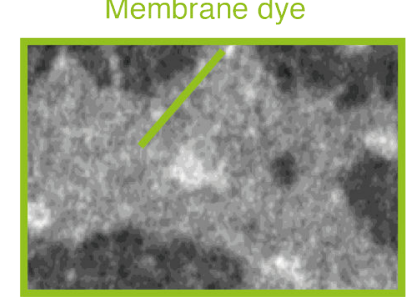

Sx-1TMD
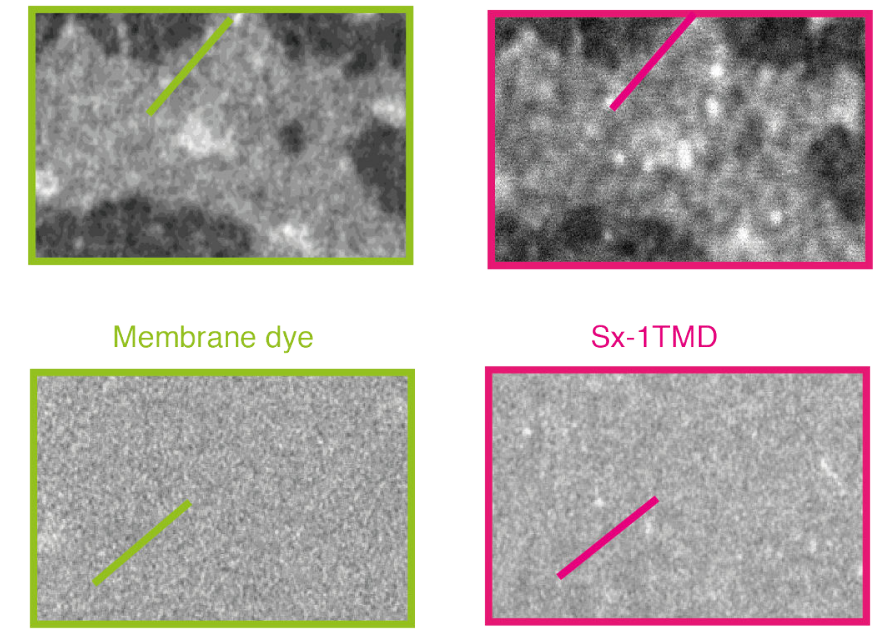

D

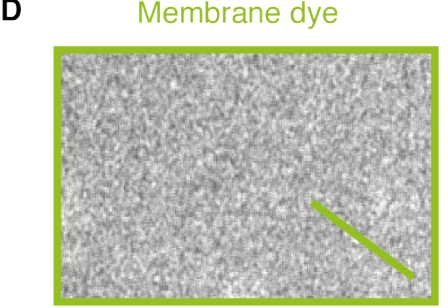

E

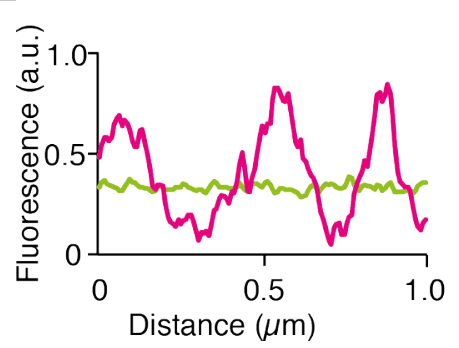

Sx-1TMD

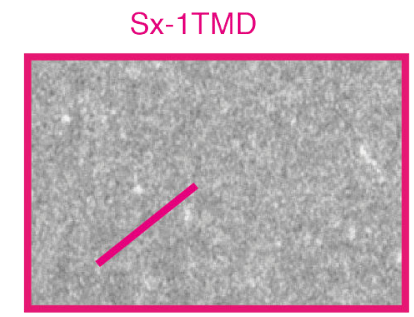

Sx-1TMD

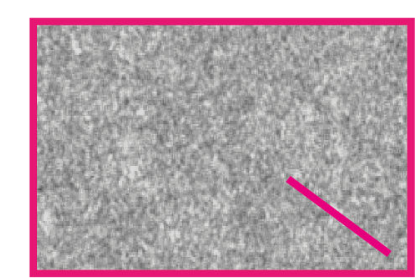

$\mathbf{F}$

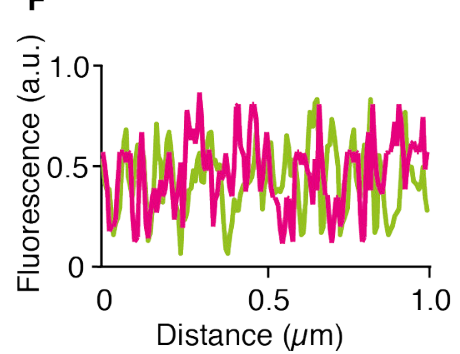

Overlay

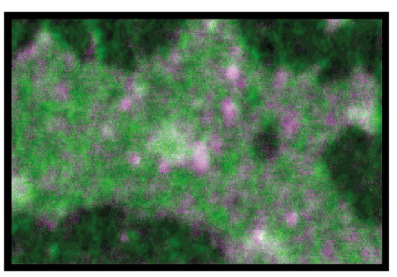

Overlay

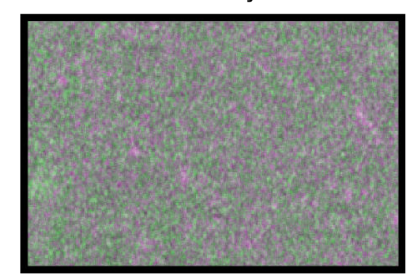

Overlay
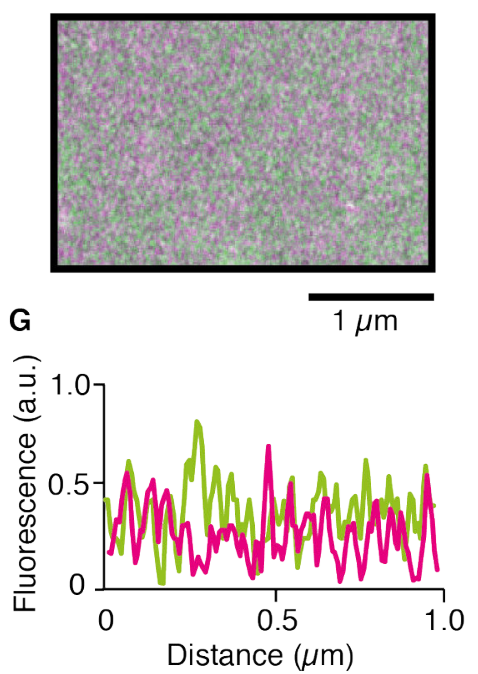

Figure 3.15. Calcium promoted syntaxin clustering requires $\mathrm{PI}(4,5) \mathrm{P}_{2}$. A. Domain organization of syntaxin 1 . The polybasic patch juxtaposed to the transmembrane domain is marked in red. B. Two-color STED images of reconstituted membranes composed of $97 \mathrm{~mol} \%$ DOPC and $3 \mathrm{~mol} \% \mathrm{PI}(4,5) \mathrm{P}_{2}$; sx-1 TMD labeled with Atto647N (magenta) and ceramide-Atto594 (membrane dye, green). Sx-1 TMD was added to a total of 1:10,000 protein-tolipid ratio; buffer contained $500 \mu \mathrm{M} \mathrm{Ca}^{2+}$. C. Same as panel $\mathbf{B}$, but now with a membrane composed of pure DOPC. D. Same as panel $\boldsymbol{B}$, but now with a membrane composed of $80 \mathrm{~mol} \%$ DOPC and $20 \mathrm{~mol} \%$ DOPS. E, F, G. Fluorescence intensity profiles from $B, C$ and $D$, respectively. Scale bar, $1 \mu \mathrm{m}$. 
I then wanted to delineate the role of calcium on syntaxin clustering in precisely controllable model membranes. I employed sx-1 TMD that does not contain its entire cytosolic domains apart from the polybasic linker (Fig. 3.15A), which allows us to exclude any effect of calcium on SNARE or Habc domains on syntaxin clustering. The lipid mixtures used for membrane reconstitutions contained DOPC without cholesterol with a thickness of approximately $3.5 \mathrm{~nm}$ which displays minimal clustering caused by hydrophobic mismatch. Hence I could focus on the effect of calcium on interactions between sx-1 TMD and $\mathrm{PI}(4,5) \mathrm{P}_{2}$. In stacked lipid bilayers, 500 $\mu \mathrm{M}$ of $\mathrm{Ca}^{2+}$ clustered reconstituted sx-1 TMD provided $\mathrm{PI}(4,5) \mathrm{P}_{2}$ was present in the membrane (Figure 3.15B). I could not observe clustering of syntaxin in membranes composed of pure DOPC or a mixture of DOPC and DOPS (Fig. 3.15C,D). This implies that $\mathrm{PI}(4,5) \mathrm{P}_{2}$ was essential for $\mathrm{Ca}^{2+}$ induced clustering.

In order to determine whether the observed clusters were really lateral membrane domains and not small membrane vesicles, I used atomic force microscopy (AFM) in collaboration with the group of Iwan Schaap. For these measurements, I prepared supported lipid bilayers with sx-1 TMD on plasmatreated glass surfaces (Fig. 3.16A). When the membrane was composed of pure DOPC, scanning showed a homogenously flat surface regardless on the presence of calcium (Fig. 3.16B,D). In contrast, and comparable to the fluorescence data, clustering was clearly observed when the membranes contained $3 \mathrm{~mol} \% \mathrm{PI}(4,5) \mathrm{P}_{2}$ (Fig. 3.16C,D). Here the circular sx-1 TMD domains had a similar size distribution as sx-1 TMD domains from the STED images with an average size of $157 \pm 2.7 \mathrm{~nm}$ (Fig. 3.16E). That these sx-1 TMD clusters were lateral membrane domains, as the average height of the patches was only $4 \mathrm{~nm}$, which is far too small for any membrane vesicle. These experiments demonstrate that calcium clusters sx-1 TMD in the presence of $\mathrm{PI}(4,5) \mathrm{P}_{2}$, which is consistent with my hypothesis.

Next, I analyzed the specificity and reversibility of calcium-triggered sx-1 TMD domain formation. To this end I reconstituted sx-1 TMD in membranes that contained $3 \mathrm{~mol} \% \mathrm{PI}(4,5) \mathrm{P}_{2}$ and recorded a series of STED images of the same membrane regions, while changing the components of the buffer (Fig. 3.17A). The addition of a final concentration of $1 \mathrm{mM} \mathrm{Mg}^{2+} \mathrm{did}$ not cause any clustering of the sx-1 TMD. However, the addition of $500 \mu \mathrm{M} \mathrm{Ca}^{2+}$ immediately triggered sx-1 TMD clusters formation with sizes between 70 
and $200 \mathrm{~nm}$ (Fig. 3.17B). These domains were dependent on the presence of $\mathrm{Ca}^{2+}$ ions, since chelating the calcium with $0.5 \mathrm{M}$ EGTA fully reversed sx-1 TMD clustering. If the $\mathrm{Ca}^{2+}$ induced clustering of sx-1 TMD occurs through $\mathrm{PI}(4,5) \mathrm{P}_{2}$, the addition of $500 \mu \mathrm{M} \mathrm{Ca}^{2+}$ should triggered clustering of $\mathrm{PI}(4,5) \mathrm{P}_{2}$ itself. Indeed, when I reconstituted a $\mathrm{PI}(4,5) \mathrm{P}_{2}$ homolog with its acyl chain labeled with Atto647N I could see detect similar calcium-induced clustering (Fig. 3.18). Together, these data clearly show that $\mathrm{Ca}^{2+}$ reversibly clusters syntaxin 1 through $\mathrm{PI}(4,5) \mathrm{P}_{2}$.

A DOPC membrane $500 \mu \mathrm{M} \mathrm{Ca}^{2+}$ in buffer

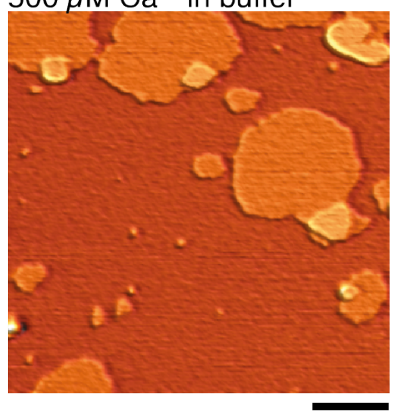

D

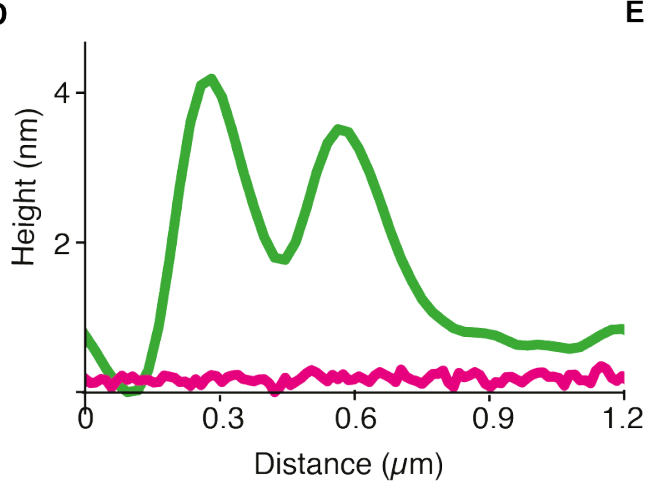

B DOPC membrane $500 \mu \mathrm{M} \mathrm{Ca}^{2+}$ in buffer

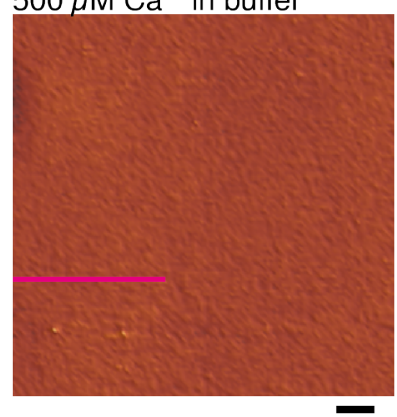

E

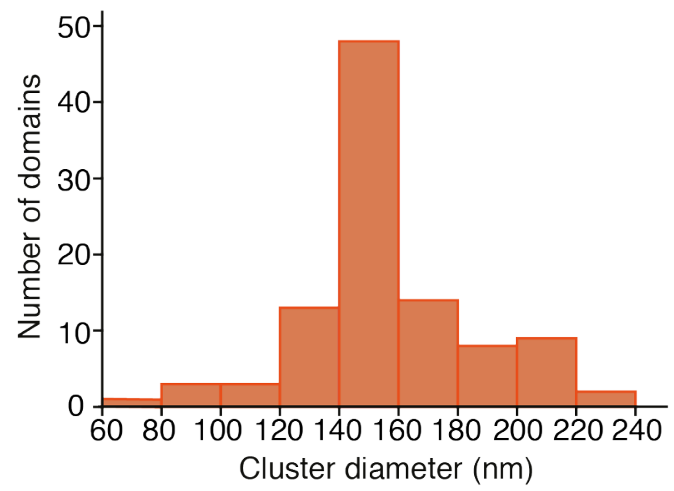

C DOPC and 3 mol\% $\mathrm{Pl}(4,5) \mathrm{P}_{2}$ $500 \mu \mathrm{M} \mathrm{Ca}^{2+}$ in buffer

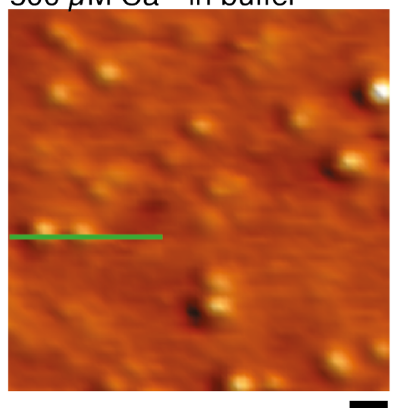

Cluster diameter $(\mathrm{nm})$

Figure 3.16. $\mathrm{Sx}-1 \mathrm{TMD} / \mathrm{PI}(4,5) \mathrm{P}_{2}$ domains reconstituted in lipid bilayers observed by AFM. A. Stacked lipid bilayers reconstituted on glass supports. Scale bar, $2 \mu \mathrm{m}$. B. Magnification of membrane region with sx-1 TMD (1:10,000 protein-to-lipid ratio) reconstituted in bilayers composed of pure DOPC. Scale bar, $0.3 \mu \mathrm{m}$. C. Same as panel $\boldsymbol{B}$, but now with bilayers containing $3 \mathrm{~mol} \% \mathrm{PI}(4,5) \mathrm{P}_{2}$. D. Line profiles from panels $B$ (pink curve) and $C$ (green). E. Size distribution of sx-1 TMD domains from $\mathrm{PI}(4,5) \mathrm{P}_{2}$ containing membranes. Data are analyzed from at least ten independent reconstitutions. 
A

A Buffer only
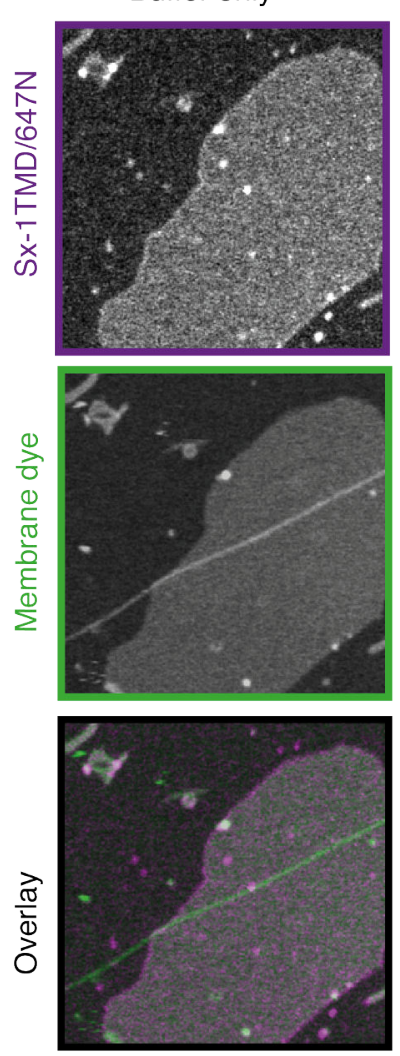

$1 \mathrm{mM} \mathrm{Mg}^{2+}$
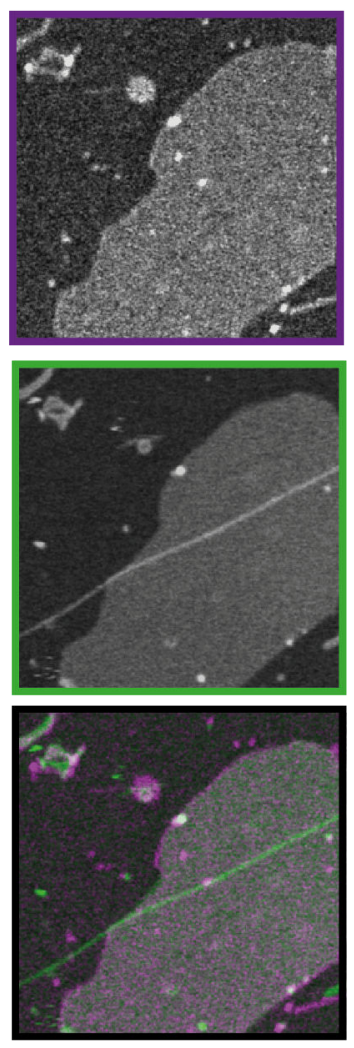

$500 \mu \mathrm{M} \mathrm{Ca}^{2+}$
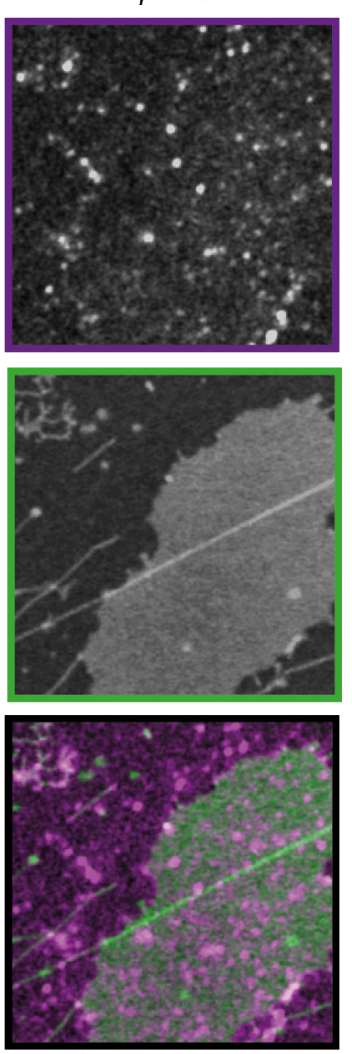

0.5 M EGTA
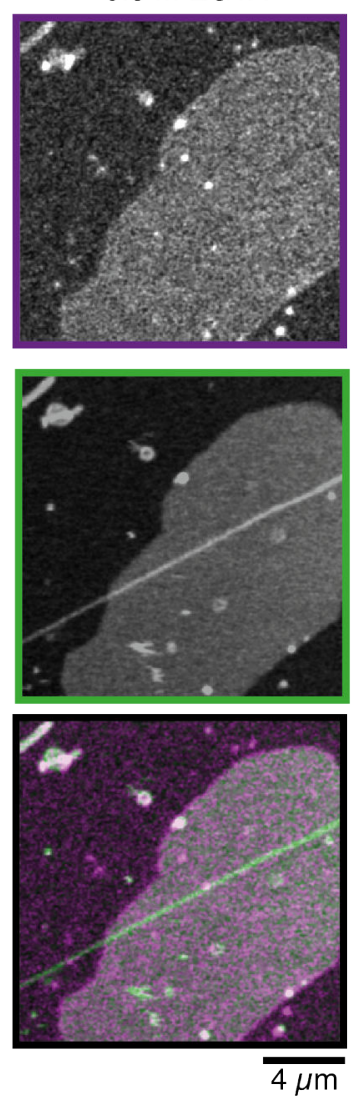

B

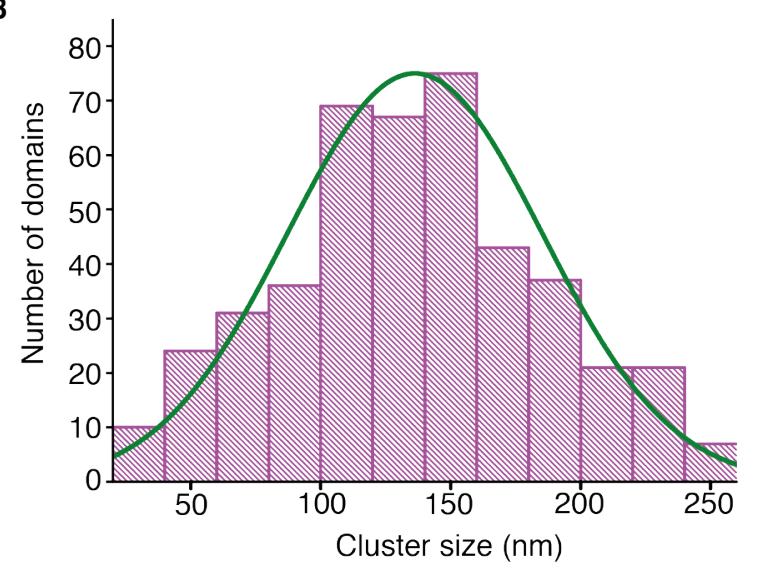

Figure 3.17. Reversible clustering of syntaxin $1 / \mathrm{PI}(4,5) \mathrm{P}_{2}$ domains by $\mathrm{Ca}^{2+}$. A. Series of STED images of reconstituted membranes composed of $97 \mathrm{~mol} \%$ DOPC and $3 \mathrm{~mol} \% \mathrm{PI}(4,5) \mathrm{P}_{2}$ with sx-1 TMD (protein-to-lipid ration was 1:10,000; magenta) and membrane dye (green). The same membrane area is

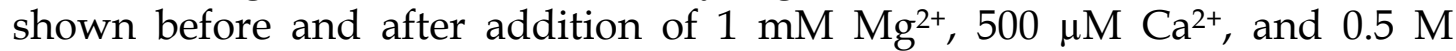
EGTA, respectively. Scale bar, $4 \mu \mathrm{m}$. B. Size distribution of sx-1 TMD domains from the membranes with $500 \mu \mathrm{M} \mathrm{Ca}^{2+}$ (purple: size distribution, green: Gaussian distribution fit). 
A

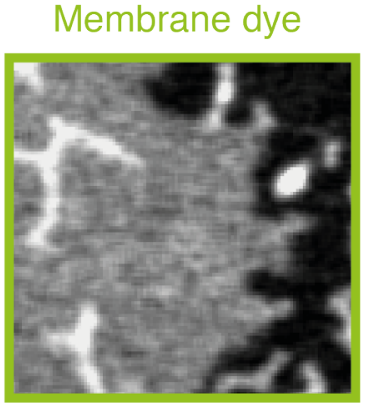

B

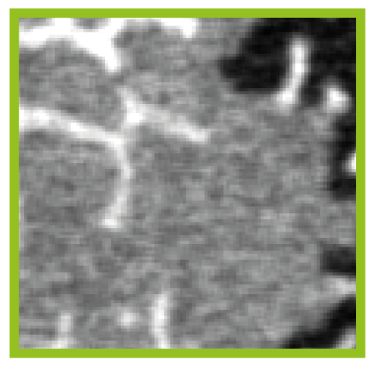

$\mathrm{PI}(4,5) \mathrm{P}_{2} /$ Atto647N
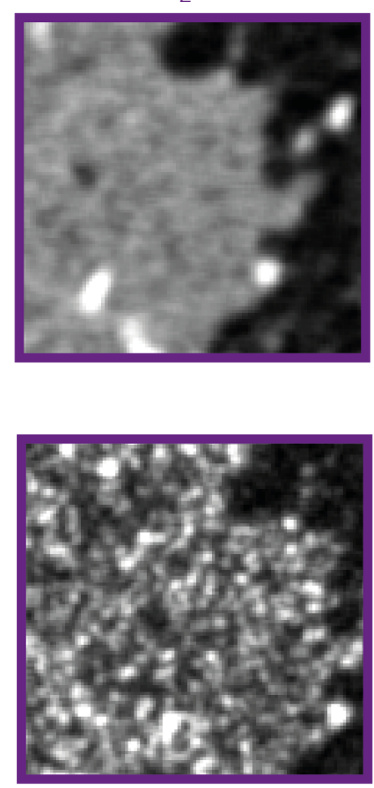

Overlay
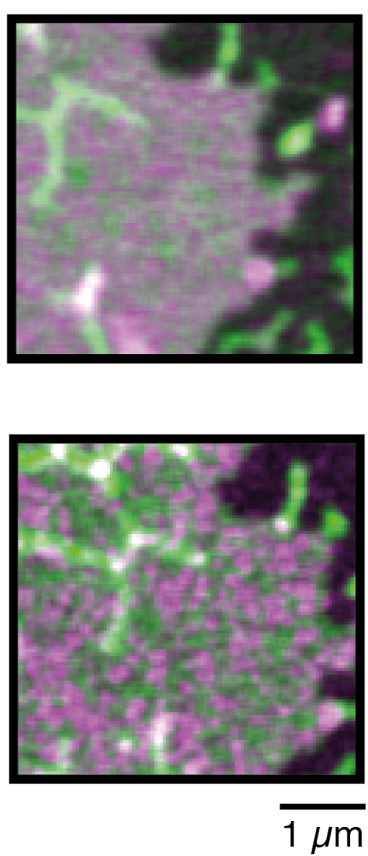

Figure 3.18. $\mathrm{Ca}^{2+}$ induces $\mathrm{PI}(4,5) \mathrm{P}_{2}$ clustering in the lipid independently of sx1 TMD. Membranes were composed of $97 \mathrm{~mol} \%$ DOPC, $3 \mathrm{~mol} \%$ unlabeled $\mathrm{PI}(4,5) \mathrm{P}_{2}, 0.1 \mathrm{~mol} \% \mathrm{PI}(4,5) \mathrm{P}_{2}$ labeled with Atto647N (magenta), and $0.1 \mathrm{~mol} \%$ DOPE-OG (green). A and B are without and with $500 \mu \mathrm{M} \mathrm{Ca}^{2+}$, respectively. Scale bar, $1 \mu \mathrm{m}$.

In order to characterize the molecular interaction between sx-1 TMD and the polar head group of $\mathrm{PI}(4,5) \mathrm{P}_{2}$, I reconstituted sx-1 TMDs (labeled with a FRET pair: RhodamineRed and Atto647N) in LUVs. I measured the emission spectra in samples before and after the addition of $150 \mu \mathrm{M} \mathrm{Ca}^{2+}$ (Fig. 3.19A). As expected, the polybasic patch of sx-1 TMD interacted with $\mathrm{PI}(4,5) \mathrm{P}_{2}$ resulting in protein clustering, and this interaction was significantly increased after the addition of $\mathrm{Ca}^{2+}$ (Fig. 3.19B). To confirm the specific interaction of the polybasic patch of sx-1 TMD with $\mathrm{PI}(4,5) \mathrm{P}_{2}$, I mutated two lysine residues located at the polybasic patch (K264A, K266A). Indeed, this sx-1 TMD mutant showed a reduced association with $\mathrm{PI}(4,5) \mathrm{P}_{2}$ and the $\mathrm{Ca}^{2+}$ effect was also diminished. Syntaxin clustering required the presence of polyvalent $\mathrm{PI}(4,5) \mathrm{P}_{2}$, as monovalent phosphatidylserine (one negative charge) did not cluster sx-1 TMD, regardless of the presence of $\mathrm{Ca}^{2+}$. Also, association between sx-1 TMD 
and $\mathrm{PI}(4,5) \mathrm{P}_{2}$ was not disrupted in the presence of PS indicating a strong electrostatic interaction between the polybasic patch and polar head-group of $\mathrm{PI}(4,5) \mathrm{P}_{2}$.

A
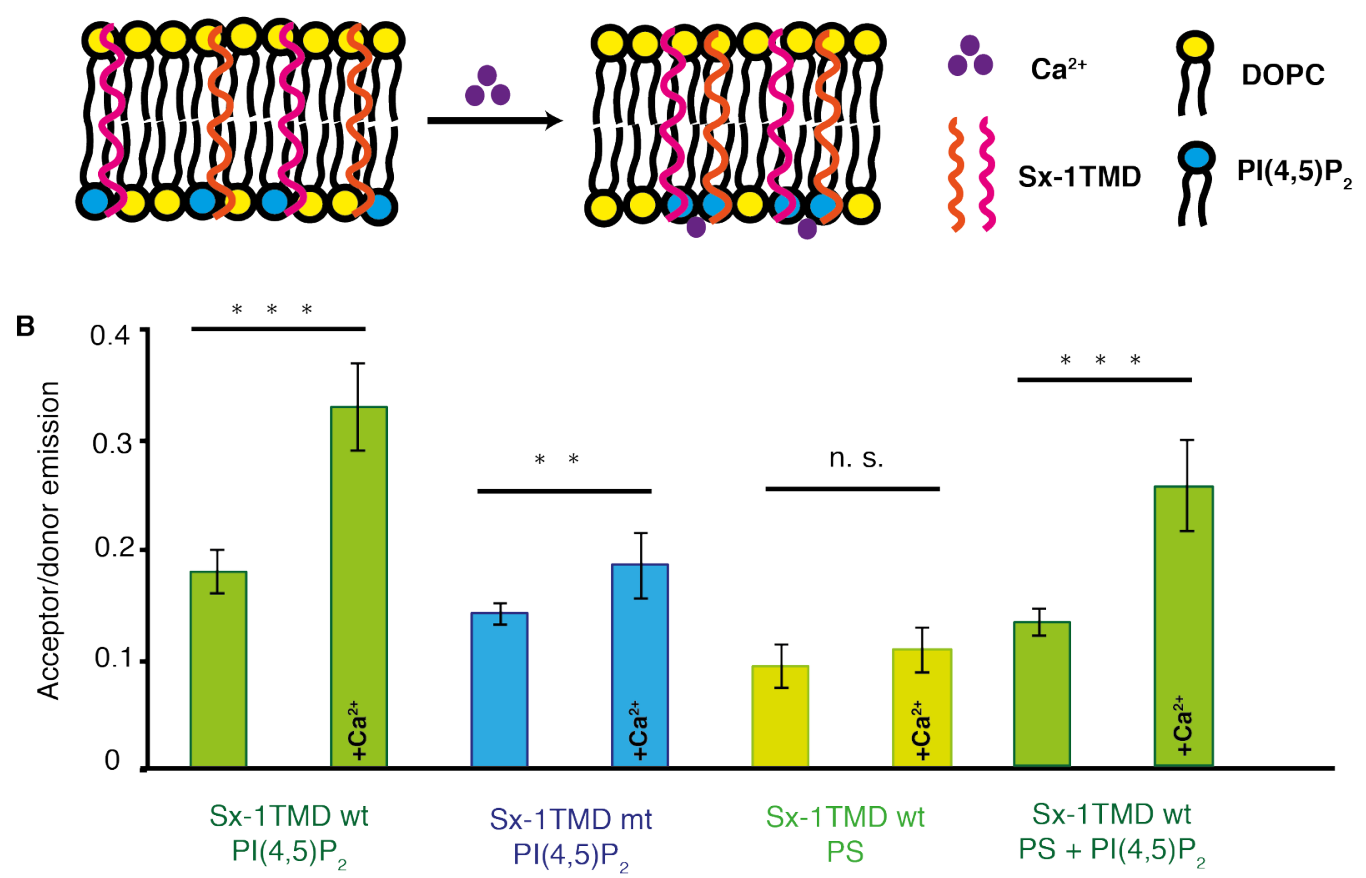

Figure 3.19. Calcium acts as charge bridge that connects multiple syntaxin $1 / \mathrm{PI}(4,5)_{2}$ complexes. A. Scheme of the FRET assay. Sx-1 TMD is labeled with Atto647N or Rhodamine Red and reconstituted in LUVs. The emission spectra were recorded before and after addition of $150 \mu \mathrm{M} \mathrm{Ca}^{2+}$. B. FRET assay shows that $150 \mu \mathrm{M} \mathrm{Ca}^{2+}$ significantly increased oligomerization of sx-1 TMD in LUVs that contain $3 \mathrm{~mol} \% \mathrm{PI}(4,5) \mathrm{P}_{2}$, but not in LUVs that contained $20 \mathrm{~mol} \%$ DOPS. Mutation of the sx-1 TMD (K264A, K265A) significantly reduced oligomerization both with and without $\mathrm{Ca}^{2+}$. Error bars: range from three independent reconstitutions, two technical repeats each.

In the final set of experiments, I analyzed the effect of $\mathrm{Mg}^{2+}$ and ATP on syntaxin clustering. These polyvalent molecules are present in the cell at relatively high concentration $\left(0.5-10 \mathrm{mM} \mathrm{Mg}^{2+}\right.$ and 1-2 $\mathrm{mM}$ ATP; Beis \& Newsholme, 1975; Hess et al., 1982; R. E. London, 1991), and they potentially could screen the charges of $\mathrm{Ca}^{2+}, \mathrm{PI}(4,5) \mathrm{P}_{2}$ and sx-1 TMD. Indeed, both $\mathrm{Mg}^{2+}$ and ATP decreased the association between sx-1 TMD and PI(4,5) $\mathrm{P}_{2}$ which is indicative for the screening of charges by these compounds (Fig. 3.20). However, $\mathrm{Ca}^{2+}$ was able to overcome this screening effect and clearly 
increased syntaxin clustering even in the presence of $\mathrm{Mg}^{2+}$ and ATP. Taken together, I conclude that $\mathrm{Ca}^{2+}$ can act as a charge bridge that brings together multiple sx-1 TMD/PI $(4,5) \mathrm{P}_{2}$ complexes into larger membrane domains.

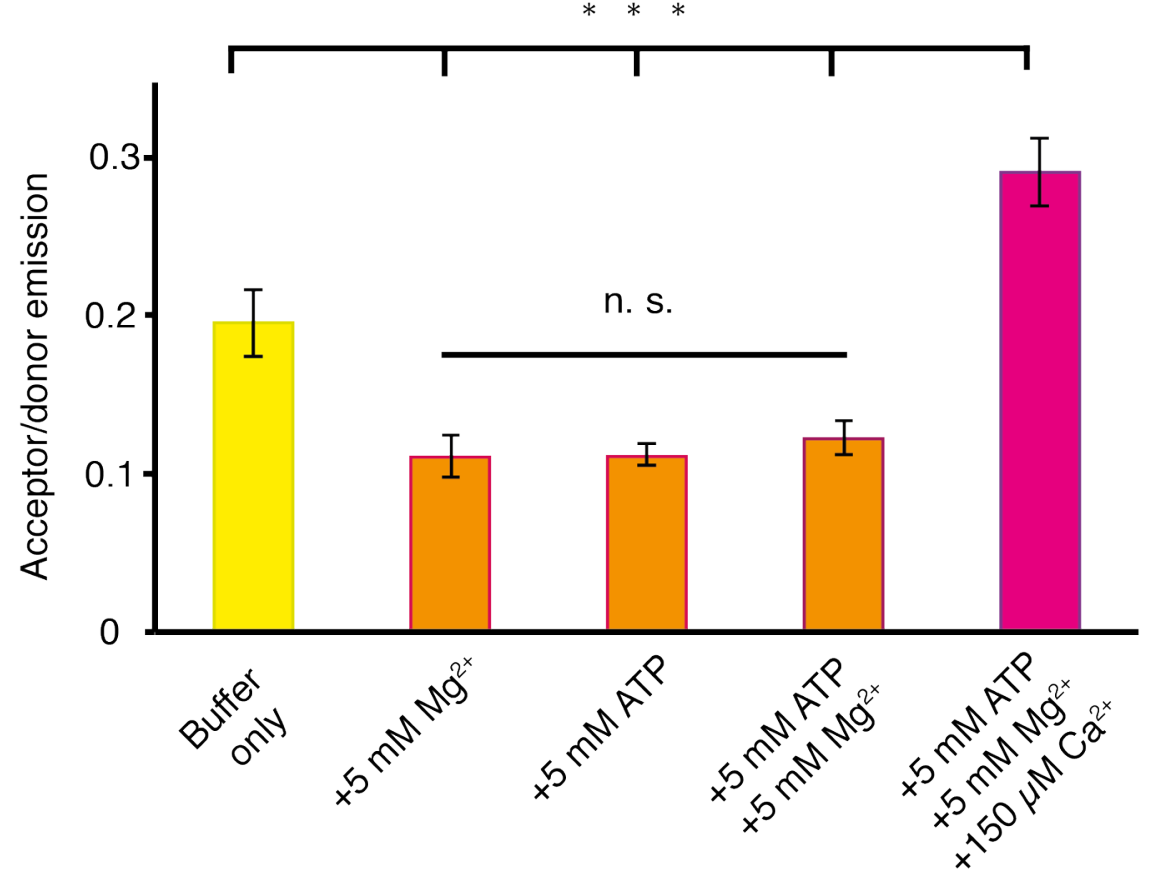

Figure 3.20. Effect of the charge screening effect on sx-1 TMD clustering in the absence and presence of $\mathrm{Ca}^{2+}$ measured by FRET. Similar to Fig. 19, FRET was

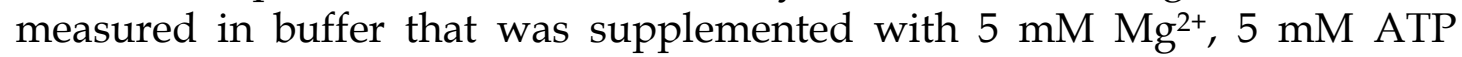
(disodium salt) or both. Finally, $150 \mu \mathrm{M} \mathrm{Ca}^{2+}$ was added to the mixture. Error bars: range from three independent reconstitutions, two technical replicates each. ${ }^{* * *} P<0.001$, two-sided, unpaired $t$-test). 


\section{DISCUSSION}

Throughout this work, I focused on understanding the organization and dynamics of SNARE proteins in the presynaptic membrane. I employed both in vitro membrane reconstitution techniques (LUVs, GUVs, polymer supported membranes) and analysis in PC12 plasma membrane sheets. First, I demonstrated that hydrophobic mismatch drives SNARE clustering and contributes to the segregation of syntaxin 1 and 4 in distinct domains in the plasma membrane. Further, I went on to integrate hydrophobic mismatch with other clustering mechanisms previously shown to contribute to SNARE clustering (i.e. protein/protein interactions and ionic protein/lipid interactions). Finally, using syntaxin clustering as a model, I elucidated a molecular mechanism of calcium-induced mesoscale domain organization.

\subsection{Difference between membrane thickness and the length of the TMDs can drive clustering of membrane proteins}

Membranes of cellular organelles have unique biochemical properties (for more details see Introduction 1.2) and some parameters such as membrane thickness vary substantially. The ER membrane is only about $3 \mathrm{~nm}$ thick, whereas the plasma membrane can be as thick as $6 \mathrm{~nm}$ (Mitra et al., 2004). The increase of membrane thickness along the secretory pathway was suggested to drive protein sorting (Munro, 1991; 1995a; 1995b). Increasing the TMD length of some resident Golgi and ER proteins causes their missorting into the plasma membrane. For instance, when the TMD of plasma membrane syntaxin 3 was truncated to be 17 instead of endogenous 23 amino acids, syntaxin 3 was retained in the cis-Golgi (Watson \& Pessin, 2001). A broad systems biology analysis of TMD length for many eukaryotic proteins indicates the clear tendency that proteins that reside in ER have a length of 17-18 amino acids and proteins in the plasma membrane contain 25-27 amino acids in their TMDs (Sharpe et al., 2010). Interestingly, plasma membrane SNAREs have 3-4 amino acids shorter TMD although their localization at the plasma membrane is critical for their physiological roles. Data presented here show that this hydrophobic mismatch between the TMD of syntaxins and the surrounding membrane is already sufficient to induce protein clustering (also 
see Milovanovic et al., 2015). Of course, SNARE clustering native membranes is multifactorial and strongly influenced by specific protein-protein and protein-lipid interactions as will be further discussed below.

It is important to emphasize that the thickness of a particular membrane is not uniform, but minor alteration in lipid distribution affects the thickness. Indeed the imaging ellipsometry data indicate that subtle increase of the acyl chain for 2 methylene groups (e.g. C14:1, C16:1, C18:1 to C20:1) increases the thickness for $0.15 \mathrm{~nm}$. Syntaxins are able to 'sense' this difference as my data show that syntaxin 1 TMD (23 amino acids long) has minimum propensity to cluster in $3.4 \mathrm{~nm}$ thick membranes, whereas syntaxin 4 TMD (24 amino acids length) has the local minimum at $3.5 \mathrm{~nm}$. Hence, the plasma membrane, with an average thickness between 4.5 and $6 \mathrm{~nm}$, can be expected to be an environment with an overall pronounced negative mismatch for syntaxins (Mitra et al., 2004; Stein et al., 2009). Moreover, because of the complex lipid composition and varying thicknesses of the plasma membrane, the difference in TMD lengths between syntaxin 1 and syntaxin 4 could at least partially contribute to their segregation into distinct regions (Sieber et al., 2006), as it will be further discussed below.

\subsection{Cholesterol increases membrane thickness and thereby increases hydrophobic mismatch}

The role of cholesterol in protein clustering is controversially discussed. SNAREs were suggested to segregate into the cholesterol/sphingomyelin rich regions, so-called detergent resistant membranes (DRM) (Chamberlain et al., 2001; Chamberlain \& Gould, 2002; Lafont et al., 1999; S. A. Predescu et al., 2005; Salaün et al., 2005). However, careful screening of different detergents indicated that SNAREs do not cluster with classical DRM markers (Lang et al., 2001; Ohara-Imaizumi, Nishiwaki, Nakamichi, et al., 2004b). Moreover, the entire concept of detergent isolation was challenged due to harsh treatments required for this procedure of low temperatures that induce phase transitions, membrane damages, and potential dehydration effects (R. F. M. de Almeida et al., 2003; Heerklotz \& Seelig, 2002; Jesús Sot et al., 2002; E. London \& Brown, 2000; Silvius et al., 1996). Nonetheless, DRMs may be indicative of clustering tendency for 
membrane proteins and they helped evolving the concept of the so-called membrane or lipid rafts (Brown \& Rose, 1992; Schroeder et al., 1994; Simons \& Ikonen, 1997; Simons \& van Meer, 1988). Lipid rafts were envisioned to represent a cellular analogue of the lipid phase-separations in model membranes (for details see Introduction 1.3). However, evidence that phaseseparation is not necessary for SNARE clustering came from analysis of syntaxin 1 and synaptobrevin 2 diffusion in model membranes composed of classical 'lipid-raft' mixtures (i.e. sphingomyelin, cholesterol and saturated phospholipids) (Bacia et al., 2004; Saslowsky et al., 2003). Here, SNAREs were shown to segregate completely into Ld phase.

However, the presence of cholesterol is essential for stability of SNARE clusters in the plasma membrane (Lang et al., 2001; Ohara-Imaizumi, Nishiwaki, Kikuta, et al., 2004a); and cholesterol extraction disrupts clusters and decreases vesicle docking (Chintagari et al., 2006; Lang et al., 2001; Ohara-Imaizumi, Nishiwaki, Nakamichi, et al., 2004b; S. A. Predescu et al., 2005). Furthermore, even $10 \mathrm{~mol} \%$ of cholesterol was shown to start inducing clustering of syntaxin 1 in LUVs and this clustering is saturated as cholesterol reaches $30 \mathrm{~mol} \%$, which corresponds to the amount present in the plasma membrane (Murray \& Tamm, 2009). These findings corroborate with results presented in this Thesis that show that cholesterol and monounsaturated phospholipids are sufficient to induce clustering of syntaxin TMDs in non-raft mixtures (i.e. without sphingomyelin and saturated phospholipids). I further show that the clustering propensity correlates not only with the inclusion of cholesterol, but also with the increase of bilayer thickness. For instance, sx-1 TMD clustering in C20:1 liposomes without cholesterol (thickness of $3.8 \mathrm{~nm}$ ) is similar to the clustering observed in C16:1 liposomes supplemented with 30 $\mathrm{mol} \%$ cholesterol (thickness of $4.0 \mathrm{~nm}$ ). Also, inclusion of $30 \mathrm{~mol} \%$ cholesterol generally increases the membrane thickness with about $0.3 \mathrm{~nm}$ (for details see Milovanovic et al., 2015). These results prove that cholesterol can cluster syntaxins by increasing the overall thickness of the membrane. This does not exclude other effects of cholesterol, but at least demonstrates that cholesterolinduced hydrophobic mismatch could be the driving force for the cholesterol dependency of SNARE clusters. 
4.3 Electrostatic interactions, protein-protein interactions and hydrophobic mismatch all modulate lateral organization of SNAREs

$\mathrm{PI}(4,5) \mathrm{P}_{2}$ is the dominant phosphoinositide lipid present in the plasma membrane (Di Paolo \& De Camilli, 2006; McLaughlin et al., 2002). Although it compromises only $1 \mathrm{~mol} \%$ of total plasma membrane lipids, it is highly enriched in particular regions of the membrane where it represent a dominant lipid (>80\%) (van den Bogaart et al., 2011). Generally, polyphosphoinositides are structurally unique since their head-groups carry polyvalent negative charges (between -4 and -7 at physiological pH) (McLaughlin et al., 2002). Early reconstitution experiments in membrane monolayers showed that the presence of $\mathrm{PI}(4,5) \mathrm{P}_{2}$ significantly decreased the diffusion coefficient of syntaxin 1 (Wagner \& Tamm, 2001). Considering that syntaxin has a polybasic patch juxtaposed to its TMDs, it was suggested that $\mathrm{PI}(4,5) \mathrm{P}_{2}$ associates with this region.

Reconstitution of syntaxin in liposomes that contained both cholesterol and $\mathrm{PI}(4,5) \mathrm{P}_{2}$ initially gave results (Murray \& Tamm, 2009; 2011) that seemed to conflict my findings. While it was clear that cholesterol alone induced syntaxins clustering, these clusters seemed to partially disperse when $\mathrm{PI}(4,5) \mathrm{P}_{2}$ was also present in the membrane, whereas I observed increased clustering. The reason for this may be the labeling of the syntaxin molecules: in these studies syntaxin was labeled at its C-terminus with Alexa 488 dye which carries a negative charge $(-2)$ at physiological pH (Murray \& Tamm, 2011). Considering that $\mathrm{PI}(4,5) \mathrm{P}_{2}$ can be expected to locate in both leaflets of the liposome bilayers, the repulsion between C-terminally labeled TMDs would compete with the association of $\mathrm{PI}(4,5) \mathrm{P}_{2}$ with the polybasic patch of syntaxin at the N-terminus. In my system, I used Rhodamine Red (no charge) and Atto647N (+1) that were both coupled to the N-terminus of the sx TMD. Hence, the overall charge balance was not disrupted and I observed that syntaxin clustering was enhanced in the presence of both cholesterol and $\mathrm{PI}(4,5) \mathrm{P}_{2}$. This is primarily an electrostatic effect, since an increase of ionic strength of the buffer screened the charge on both $\mathrm{PI}(4,5) \mathrm{P}_{2}$ and syntaxin 1 . This corroborates with biochemical and cell biology data in which the polybasic patch of syntaxin was identified as critical region for association with polyphosphoinositides (Khuong et al, 2013; van den Bogaart et al, 2011). 
In a complex plasma membrane environment, specific protein-protein interactions of the SNARE domains segregate syntaxins 1 and 4 into distinct domains (Sieber et al., 2006). However, in this study it was already clear that TMDs of syntaxin 1 and 4 have the capacity to cluster in a cholesterol dependent manner. In this Thesis, I further addressed this issue and I now show that clustering of the TMDs is driven by negative hydrophobic mismatch and that the difference of the TMD lengths contributes to the segregation of syntaxin 1 and 4 . In fact, increasing the mismatch between sx-1 and sx- 4 TMDs to 5 amino acids difference segregates these constructs apart to a similar extent as the full-length constructs (see also Milovanovic et al., 2015). This implies that the ionic interactions and the average plasma membrane thickness (i.e. between 4-6 nm) induce a strong propensity for clustering of syntaxin isoforms. However, specific protein-protein interactions and the slight differences in the TMD lengths can segregate syntaxins apart (Fig. 4.1).

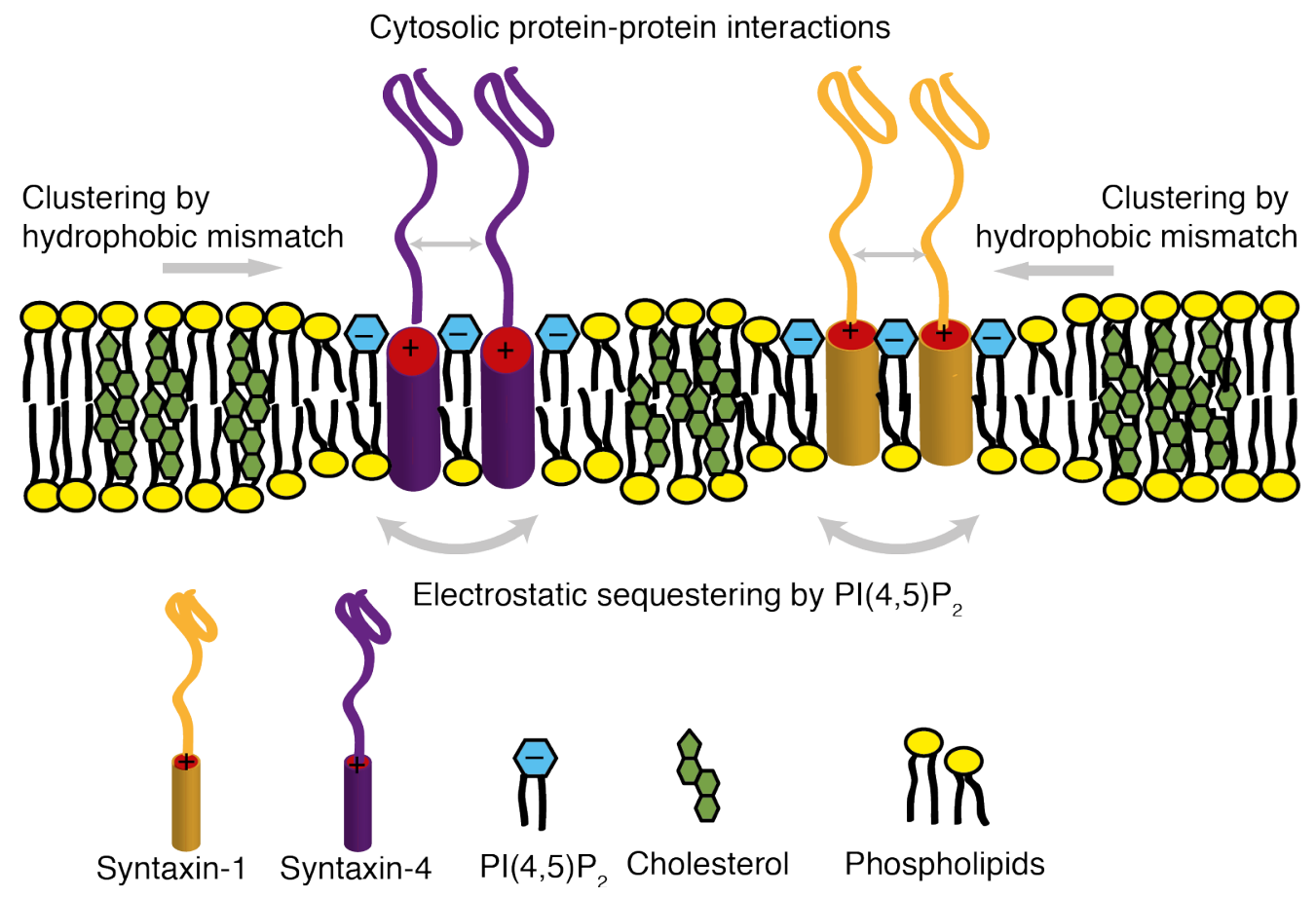

Figure 4.1. Synergistic model of syntaxin clustering in the membrane. The lateral organization of SNAREs is modulated by hydrophobic mismatch, electrostatic interactions and protein-protein interactions. Adapted from Milovanovic et al., 2015. 
It is important to emphasize that SNARE clusters are heterogeneous in their composition. For instance, syntaxin 1 associates with polyphosphoinositides (Khuong et al., 2013; van den Bogaart et al., 2011) as well as with SNAP 25 (Bar-On et al., 2012; Rickman et al., 2010) and many of the accessory proteins (Barg et al., 2010; Gandasi \& Barg, 2014). Also, proteins and lipids diffuse freely between these clusters (Barg et al., 2010; Knowles et al., 2010; Sieber et al., 2007). According to Saffman-Delbrück (SD) lateral diffusion model (Saffman \& Delbrück, 1975), doubling the radius of a protein in a bilayer decreases its lateral diffusion coefficient only by approximately $20 \%$. Although SD model was initially developed to explain the protein mobility in the membrane (Weiß et al., 2013), experiments and calculations showed that it can be further used for following the diffusion of membrane inclusions (i.e. clusters) (Cicuta, Keller, \& Veatch, 2007; Petrov \& Schwille, 2008).

In a very sensitive assay where GUVs were spread on porous substrate generating free standing membranes (so-called pore spanning membranes; developed by group of Prof. Steinem, University of Göttingen), we observed that the diffusion coefficients of full length syntaxin $1\left(\sim 2.3 \mu \mathrm{m}^{2} \mathrm{~s}^{-1}\right)$ decrease comparing to the sx-1 TMD $\left(\sim 3.4 \mu \mathrm{m}^{2} \mathrm{~s}^{-1}\right)$ for about $40 \%$ (Schwenen et al, 2015). Since the lipid mixtures in this assay contained cholesterol (i.e. the membranes had a substantial hydrophobic thickness), we conclude that clustering is not only driven by hydrophobic thickness but that proteinprotein interactions further increase the clustering. Given the structural heterogeneity of the SANRE clusters, it is exactly the competition between different protein and lipid interactions that leads to their dynamic equilibrium (i.e. constant remodeling) and allows the proteins and lipids to freely diffuse between the clusters. 


\section{4 $\mathrm{Ca}^{2+}$ acts as a charge bridge that connects multiple syntaxin $1 / \mathrm{PI}(4,5) \mathrm{P}_{2}$ clusters into mesoscale domains}

It was recently shown that calcium ions increase clustering of SNAREs in the plasma membrane (Zilly et al., 2011). This calcium induced increase of protein clustering and formation of larger domains of sizes above $200 \mathrm{~nm}$ (socalled mesoscale domains) was suggested to be a consequence of overall negative net charge of SNAREs. The suggested mechanism indicated that $\mathrm{Ca}^{2+}$ could crosslink aspartate and glutamate residues at the surface of SNAREs (Zilly et al., 2011). However, it is well established that $\mathrm{Ca}^{2+}$ can also cluster $\mathrm{PI}(4,5) \mathrm{P}_{2}$ by acting as charge bridge, connecting multiple $\mathrm{PI}(4,5) \mathrm{P}_{2}$ molecules together (Carvalho, Ramos, Roy, \& Picart, 2008; Ellenbroek et al., 2011; Levental et al., 2009; Y.-H. Wang et al., 2012; 2014). Therefore, I hypothesized that calcium would promote syntaxin 1 domain formation through the interactions with $\mathrm{PI}(4,5) \mathrm{P}_{2}$. Indeed, my data show that calcium clusters truncated mutants of syntaxin lacking almost the entire cytoplasmic domain, but only provided $\mathrm{PI}(4,5) \mathrm{P}_{2}$ is present in the membrane.

Interestingly, only $\mathrm{Ca}^{2+}$ and not $\mathrm{Mg}^{2+}$ promote membrane clustering of syntaxin $1 / \mathrm{PI}(4,5) \mathrm{P}_{2}$. These findings correlate well with several chemistry studies that characterized the interactions between divalent cations and $\mathrm{PI}(4,5) \mathrm{P}_{2}$ and showed that $\mathrm{Ca}^{2+}$ specifically causes $\mathrm{PI}(4,5) \mathrm{P}_{2}$ domain formation (Levental et al., 2009; Y.-H. Wang et al., 2012). In contrast to these studies, my data show that $\mathrm{Ca}^{2+} / \mathrm{PI}(4,5) \mathrm{P}_{2}$ association also occurs at physiological concentrations of $\mathrm{PI}(4,5) \mathrm{P}_{2}$ and $\mathrm{Ca}^{2+}$. The $\mathrm{Ca}^{2+}$ specificity is due to the charge density distribution and matching of chelating properties between $\mathrm{Ca}^{2+}$ and the polynegative head-group of $\mathrm{PI}(4,5) \mathrm{P}_{2}$ (Sarmento, Coutinho, Fedorov, Prieto, \& Fernandes, 2014; Y.-H. Wang et al., 2012; 2014). This implies that $\mathrm{Ca}^{2+}$ acts as the electrostatic bridge that connects syntaxin 1 molecules indirectly via $\mathrm{PI}(4,5) \mathrm{P}_{2}$ (Fig. 4.2). Indeed, the surface interactions between syntaxin 1 and $\mathrm{PI}(4,5) \mathrm{P}_{2}$ are of electrostatic nature since they are strongly sensitive to the presence of ATP and $\mathrm{Mg}^{2+}$. These molecules non-specifically screen the charges on the polybasic patch of syntaxin 1 and the head-group of $\mathrm{PI}(4,5) \mathrm{P}_{2}$, thereby reducing the surface interactions between lipids and proteins (similarly as in Park et al., 2012). Despite this screening, $\mathrm{Ca}^{2+}$ can still 
cause clustering of syntaxin $1 / \mathrm{PI}(4,5) \mathrm{P}_{2}$, further demonstrating that this must be a specific electrostatic interaction. Altogether, these results confirm that $\mathrm{Ca}^{2+}$ can specifically and reversibly induce mesoscale domains of syntaxin 1 and $\mathrm{PI}(4,5) \mathrm{P}_{2}$ in the plasma membrane.

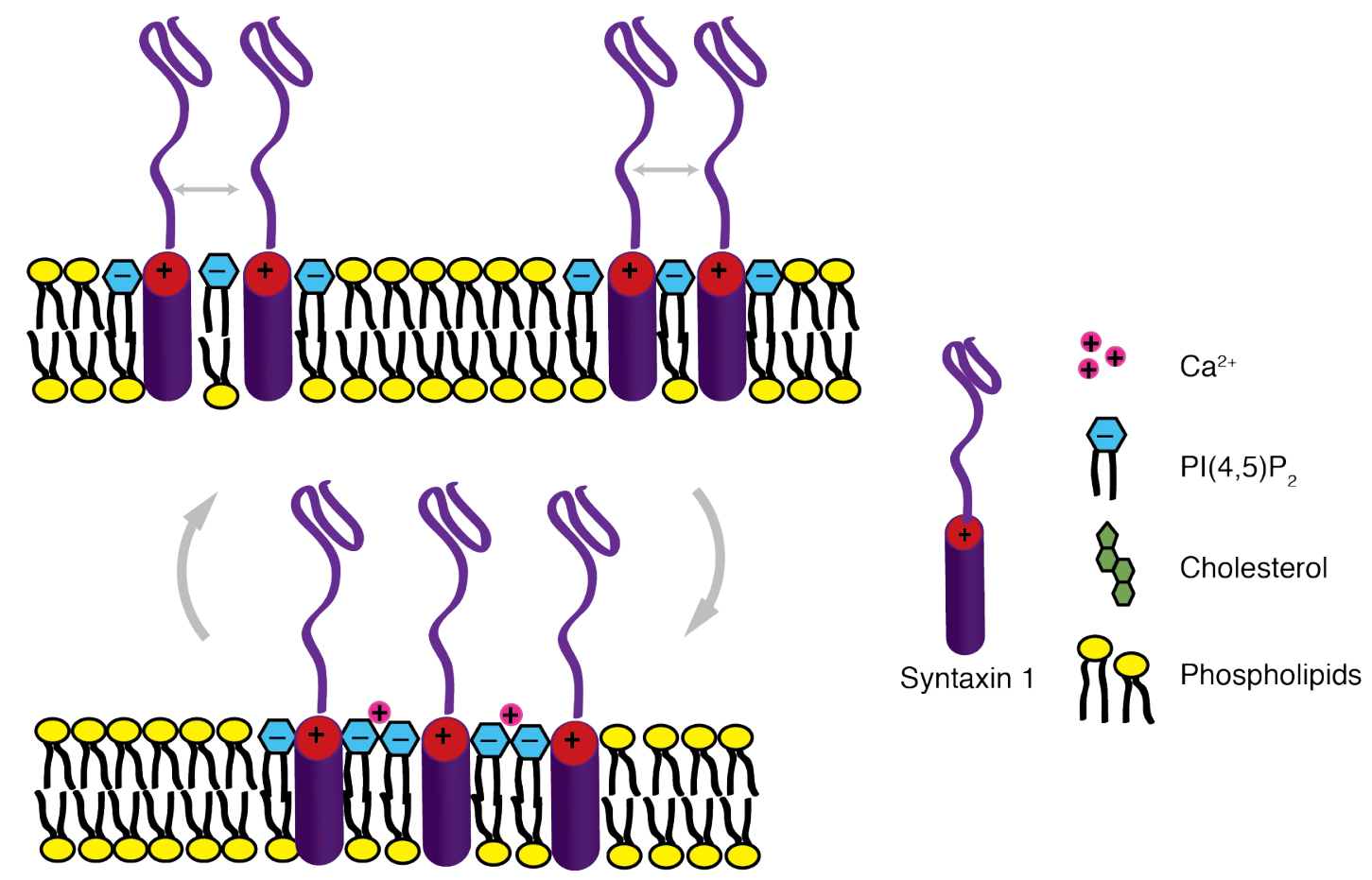

$\mathrm{Ca}^{2+}-$ induced mesoscale domain

Figure 4.2. Model of calcium-induced syntaxin domain formation. $\mathrm{Ca}^{2+}$ acts as a charge bridge that connects multiple syntaxin $1 / \mathrm{PI}(4,5) \mathrm{P}_{2}$ oligomers into clusters.

\subsection{3hysiological significance of SNARE clustering in the plasma membrane}

SNARE clusters in the plasma membrane can act as local hot spots for vesicle docking, they may promote vesicle fusion and help in maintaining the organelle identity during endocytosis and subsequent vesicle sorting.

Attachment of synaptic vesicles to the plasma membrane is a broadly discussed topic and intensive research efforts indicate that there may be more than one tethering/docking determinant (for detailed discussion of current models, see: Chapman, 2008; Hong \& Lev, 2014; Imig et al., 2014; Jahn \& 
Fasshauer, 2012; Rizo \& Südhof, 2012). For instance, synaptotagmin 1, the main calcium sensor of exocytosis, has been proposed the to contribute to docking of vesicles (de Wit et al., 2009; Milosevic et al., 2005). Synaptotagmin 1 is a single spanning vesicular protein that contains two C2 domains (C2A and $\mathrm{C} 2 \mathrm{~B}$ ) that bind to three and two $\mathrm{Ca}^{2+}$ ions, respectively (Chapman, 2008; Fernandez et al., 2001; Radhakrishnan, Stein, Jahn, \& Fasshauer, 2009). Additionally, the $\mathrm{C} 2 \mathrm{~B}$ domain contains the polybasic patch that could bind to negative regions at the membrane surface (Araç et al., 2006; Bai, Tucker, \& Chapman, 2004; Herrick, Sterbling, Rasch, Hinderliter, \& Cafiso, 2006; Stein, Radhakrishnan, Riedel, Fasshauer, \& Jahn, 2007; Vennekate et al., 2012).

Indeed, we showed that synaptotagmin 1 binds to syntaxin1/ $\mathrm{PI}(4,5) \mathrm{P}_{2}$ patches in calcium-independent manner (Honigmann et al., 2013). Here, the calcium-binding pockets were free to bind to acidic phospholipids in either the vesicular or plasma membrane and $\mathrm{PI}(4,5) \mathrm{P}_{2}$ was shown to increase synaptotagmin 1 affinity for $\mathrm{Ca}^{2+}$ (van den Bogaart, Meyenberg, Diederichsen, \& Jahn, 2012), which may partially explain the high calcium sensitivity at the exocytotic sites (Sakaba, Schneggenburger, \& Neher, 2002; Schneggenburger \& Neher, 2005).

Alternatively, synaptotagmin 1 has been suggested to transiently bind to the negative surface of SNARE complex in a calcium-independent manner (Araç et al., 2006; Brewer et al., 2015; Rizo, Chen, \& Araç, 2006). In this case, synaptotagmin 1 could putatively interrupt the full zippering of a SNARE complex (Bhalla, Chicka, Tucker, \& Chapman, 2006; Tang et al., 2006) and only upon calcium entry and its displacement the full zippering would be allowed. However, most of these data are obtained under non-physiological ion strength (i.e. low salt conditions) and deeper biochemical characterization needs to be done under physiological conditions (for discussion see Jahn \& Fasshauer, 2012; Rizo \& Südhof, 2012).

It is important to emphasize that the neuronal synapses contain precisely organized active zone (Fernández-Busnadiego et al., 2010; 2013; Harlow, Ress, Stoschek, Marshall, \& McMahan, 2001; Szule et al., 2012) in contrast to PC12 cells where the vesicle release occurs over the entire membrane. Hence, although syntaxin $1 / \mathrm{PI}(4,5) \mathrm{P}_{2}$ domains may act as 'molecular beacons' and recruit vesicle to the membrane of PC12 cells (de Wit et al., 2009; Honigmann et al., 2013), in neuronal exocytosis synaptotagmin 1 
recognition of syntaxin $1 / \mathrm{PI}(4,5) \mathrm{P}_{2}$ may be dispensable (Imig et al., 2014). Accessory proteins such as Munc 18 are able to peal syntaxin molecules from the clusters and further engage in vesicle recruitment (Bar-On et al., 2012; Gandasi \& Barg, 2014) as well as in fusion complex formation (Ma, Su, Seven, Xu, \& Rizo, 2013).

SNARE clusters may also facilitate the fusion reaction. Although a single (Mohrmann et al., 2010; Shi et al., 2012; van den Bogaart et al., 2010) or just a few (Mohrmann et al., 2010; Sinha, Ahmed, Jahn, \& Klingauf, 2011) SNARE complexes are needed for fusion of synaptic vesicles, sustained exocytosis would require a pool of easily accessible proteins. Here, SNARE clusters are the protein buffer for fusion complex formation. Additionally, it is emerging that the fusion of tightly docked vesicles could be triggered by lipid fluctuations (Risselada et al., 2014; Risselada \& Grubmüller, 2012). Our molecular dynamics simulations (collaboration with Herre Jelger Risselada and Helmut Grubmüller) show that cholesterol-induced hydrophobic mismatch causes a corresponding free energy penalty proportional to the protein-lipid interface (Milovanovic et al., 2015). Assuming that the effective TMD extension of the mismatch interface is constant, the length of proteinlipid interface determines the free energy penalty. Such an energy penaltyper-TMD length, also referred to as line tension, makes the surrounding lipids more susceptible to fluctuation between the bilayers (Kozlov et al., 2014; Risselada et al., 2014). Given that the plasma membrane contains lipids of different shape (for details see Introduction), enhanced lipid fluctuations makes the remodeling of the plasma membrane easier.

SNARE clustering may further be important for maintaining the organelle identity during the vesicle cycle. Detailed quantitative analyses of protein and lipid components in synaptic vesicles and synapses shows that SNAREs are highly abundant proteins (i.e. $>20,000$ copies of syntaxin 1 and SNAP 25 in the entire synapse) (Wilhelm et al., 2014). Some proteins at synaptic vesicles are abundant such as synaptobrevin 2 ( 70 copies/vesicle) whereas some are present at only $1-2$ copies/vesicle, e.g. the vacuolar ATPase required for neurotransmitter uptake (Takamori et al., 2006). Considering that synaptic vesicle release requires only two synaptobrevin 2 molecules (Mohrmann et al., 2010; Shi et al., 2012; Sinha et al., 2011), some flexibility in the copy-number of SNAREs in the vesicle may be tolerated. 
However, the repetitive and fast process of neurotransmitter release requires fast membrane turnover (Rizzoli, 2014). Upon exocytosis, SNAREs were shown to either remain clustered (Willig et al., 2006) or quickly re-cluster prior to the endocytosis (Hua et al., 2011). Based on previous observations from our lab (Zilly et al., 2011) and data presented here that calcium stimulates syntaxin clustering, it is tempting to speculate that these clustering mechanism may help plasma membrane SNARE sorting to facilitate fast endocytosis.

Moreover, synaptic vesicle proteins such as synaptotagmin remain clustered even within the early endosome (Hoopmann et al., 2010), where they serve as a marker for synaptic vesicle retrieval (S. Watanabe, Trimbuch, et al., 2014b). In case of clathrin mediated endocytosis specific adaptor proteins assure that synaptic vesicle components cluster in a coated pit (Collins et al., 2002; Glyvuk et al., 2010; Jung et al., 2007; for details see Introduction 1.4). The protein and lipid content of these endocytic compartments might well match that of synaptic vesicles, which would allow their direct reuse. However, especially in case of sustained synaptic activity which would ultrafast endocytosis (S. Watanabe, Liu, et al., 2013a; S. Watanabe, Rost, et al., 2013b) or clathrin-independent uptake, the freshly engulfed membranes would have to be proofed in order to determine whether the vesicle has to undergo additional sorting in the endosome or whether it may directly re-enter the SV pool. For a better understanding of vesicle turnover, the sorting signals and proofreading components in the membrane need to be better determined. Based on my findings, I propose that protein/lipid clusters may well be important to segregate plasma membrane proteins apart from synaptic vesicle proteins, as well as for the proofreading whether newly endocytosed vesicles meet the requirements for functional synaptic vesicles. 


\section{CONCLUSIONS and PERSPECTIVES}

From this study I can conclude that a single mechanism cannot explain the diversity of different clusters in the membrane. It is the interplay, and one would dare to say - the competition, between different mechanisms that establishes particular clustering pattern (Fig. 5.1). Also, some parameters (for instance, protein-protein interaction in the case of SNAREs) may be dominant for segregation of these proteins in particular clusters despite of the local thermodynamic properties of the membrane. Additionally, cytosolic factor (e.g. ions and charged small molecules) can further fine-tune the protein/protein and protein/lipid interactions in the membrane.

Cellular membranes should not be envisioned as smooth structures, but rather their thickness varies according to the local lipid and protein environment. The high abundance of proteins in the membrane (i.e. more that $20 \mathrm{~mol} \%$ of the total bilayer volume are TMDs; Dupuy \& Engelman, 2008; Takamori et al., 2006) warrants them to be seen as a phase itself and biochemical properties of these TMDs may substantially contribute to membrane structure. Finally, protein and lipid clusters are in constant dynamic equilibrium with the proteins that freely diffuse between these clusters. In fact, it is exactly these regions between the clusters that may act as hot spots for biological processes such as exo- and endocytosis. Beyond their role in the vesicle cycle, SNAREs are the main targets of Clostridial neurotoxins (Rossetto, Pirazzini, \& Montecucco, 2014) and even the general anesthetics appear to disrupt their organization (Herring et al., 2011; Xie et al., 2013).

In this Thesis, SNAREs were used as a paradigm to understand the structure and dynamics of membrane domains. Described principles may apply to the other proteins and lipids. It is important to foster our knowledge on membrane structure and dynamics, because membranes play a critical role for a range of cellular functions such as adhesion, tissue formation, cell proliferation, migration, as well as in intracellular trafficking and organelle cross talk. 
Until now understanding of membrane domains was largely confined to the plasma membrane. In the future it will be interesting to understand if the same protein and lipid organizing principles also apply to organelles where they may play a role in processes such as sorting during trafficking and in organelle biogenesis.

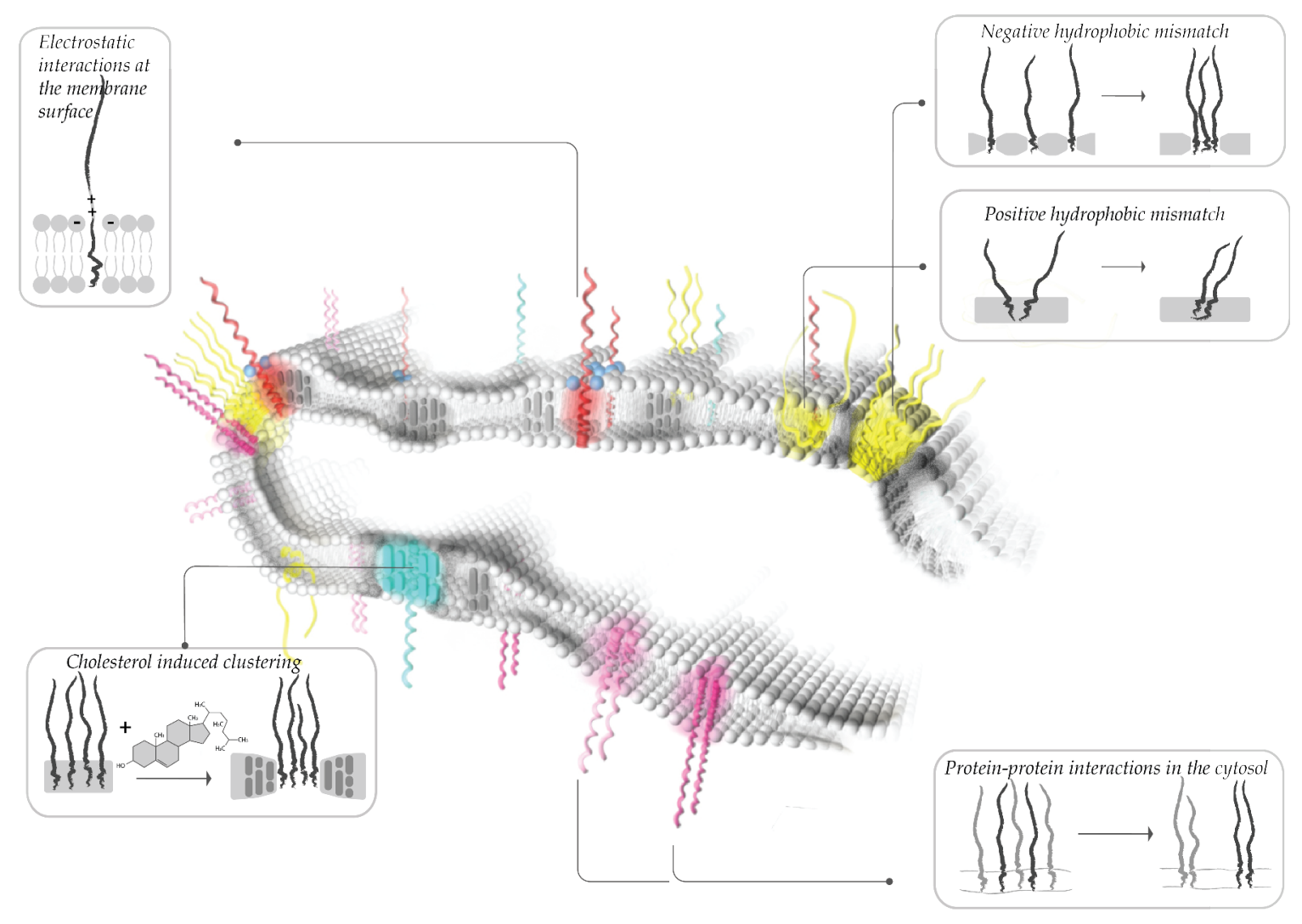

Figure 5.1. Biological membranes are modular systems: different mechanisms contribute to the lateral organization of the membrane and allow for the dynamic instability of protein and lipid domains. Adapted from Milovanovic \& Jahn, 2015. 


\section{REFERENCES}

Abbe, E. (1873) Beiträge zur Theorie des Mikroskops und der mikroskopischen Wahtnehmung. Arch. Mikroskop. Anat. 9, 413-420.

Aittoniemi, J., Niemelä, P. S., Hyvönen, M. T., Karttunen, M., \& Vattulainen, I. (2007). Insight into the Putative Specific Interactions between Cholesterol, Sphingomyelin, and Palmitoyl-Oleoyl Phosphatidylcholine. Biophysical Journal, 92(4), 1125-1137.

Anderson, R. G. W., \& Jacobson, K. (2002). A role for lipid shells in targeting proteins to caveolae, rafts, and other lipid domains. Science, 296(5574),

Antonin, W., Fasshauer, D., Becker, S., Jahn, R., \& Schneider, T. R. (2002). Crystal structure of the endosomal SNARE complex reveals common structural principles of all SNAREs. Nature Structural Biology, 9(2), 107.

Aoyagi, K., Sugaya, T., Umeda, M., Yamamoto, S., Terakawa, S., \& Takahashi, M. (2005). The activation of exocytotic sites by the formation of phosphatidylinositol 4,5-bisphosphate microdomains at syntaxin clusters. Journal of Biological Chemistry, 280(17), 17346-17352.

Araç, D., Chen, X., Khant, H. A., Ubach, J., Ludtke, S. J., Kikkawa, M., et al. (2006). Close membrane-membrane proximity induced by $\mathrm{Ca}(2+)-$ dependent multivalent binding of synaptotagmin-1 to phospholipids. Nature Structural \& Molecular Biology, 13(3), 209-217.

Atkins, P., de Paula, J. (2014) Atkins' Physical Chemistry. 10 ${ }^{\text {th }}$ Edition, Oxford University Press

Bacia, K., Haustein, E., \& Schwille, P. (2014). Fluorescence correlation spectroscopy: principles and applications. Cold Spring Harbor Protocols, 2014(7), 709-725.

Bacia, K., Schuette, C. G., Kahya, N., Jahn, R., \& Schwille, P. (2004). SNAREs prefer liquid-disordered over "raft" (liquid-ordered) domains when reconstituted into giant unilamellar vesicles. Journal of Biological Chemistry, 279(36), 37951-37955.

Bagatolli, L. A., Ipsen, J. H., Simonsen, A. C., \& Mouritsen, O. G. (2010). An outlook on organization of lipids in membranes: Searching for a realistic connection with the organization of biological membranes. Progress in Lipid Research, 49(4), 378-389.

Bai, J., Tucker, W. C., \& Chapman, E. R. (2004). PIP2 increases the speed of response of synaptotagmin and steers its membrane-penetration activity toward the plasma membrane. Nature Structural $\mathcal{E}$ Molecular Biology, 11(1), 36-44.

Bar-On, D., Wolter, S., van de Linde, S., Heilemann, M., Nudelman, G., 
Nachliel, E., et al. (2012). Super-resolution imaging reveals the internal architecture of nano-sized syntaxin clusters. Journal of Biological Chemistry, 287(32), 27158-27167.

Barg, S., Knowles, M. K., Chen, X., Midorikawa, M., \& Almers, W. (2010). Syntaxin clusters assemble reversibly at sites of secretory granules in live cells. Proceedings of the National Academy of Sciences of the United States of America, 107(48), 20804-20809.

Beis, I., \& Newsholme, E. A. (1975). The contents of adenine nucleotides, phosphagens and some glycolytic intermediates in resting muscles from vertebrates and invertebrates. Biochemical Journal, 152(1), 23-32.

Bennett, V., \& Baines, A. J. (2001). Spectrin and ankyrin-based pathways: metazoan inventions for integrating cells into tissues. Physiological Reviews, 81(3), 1353-1392.

Berne, B. J., \& Pecora, R. (2000). Dynamic Light Scattering: With Applications to Chemistry, Biology, and Physics. Dover Publications.

Bhalla, A., Chicka, M. C., Tucker, W. C., \& Chapman, E. R. (2006). Ca(2+)synaptotagmin directly regulates t-SNARE function during reconstituted membrane fusion. Nature Structural \& Molecular Biology, 13(4), 323-330.

Bhattacharyya, R., Barren, C., \& Kovacs, D. M. (2013). Palmitoylation of amyloid precursor protein regulates amyloidogenic processing in lipid rafts. The Journal of Neuroscience, 33(27), 11169-11183.

Bigay, J., \& Antonny, B. (2012). Curvature, lipid packing, and electrostatics of membrane organelles: defining cellular territories in determining specificity. Developmental Cell, 23(5), 886-895.

Blodgett, K. B. (1935). Films built by depositing successive monomolecular layers on a solid surface. Journal of the American Chemical Society, 57(6),

Boucrot, E., Pick, A., Çamdere, G., Liska, N., Evergren, E., McMahon, H. T., \& Kozlov, M. M. (2012). Membrane fission is promoted by insertion of amphipathic helices and is restricted by crescent BAR domains. Cell, 149(1), 124-136.

Boukh-Viner, T., \& Titorenko, V. I. (2006). Lipids and lipid domains in the peroxisomal membrane of the yeast Yarrowia lipolytica. Biochimica Et Biophysica Acta (BBA) - Molecular Cell Research, 1763(12), 1688-1696.

Brandenburg, K., Garidel, P., Howe, J., Andrä, J., Hawkins, L., Koch, M. H. J., \& Seydel, U. (2006). What can calorimetry tell us about changes of threedimensional aggregate structures of phospholipids and glycolipids? Thermochimica Acta, 445(2), 133-143.

Bretscher, A., Edwards, K., \& Fehon, R. G. (2002). ERM proteins and merlin: integrators at the cell cortex. Nature Reviews Molecular Cell Biology, 3(8), 
586-599.

Brewer, K. D., Bacaj, T., Cavalli, A., Camilloni, C., Swarbrick, J. D., Liu, J., et al. (2015). Dynamic binding mode of a Synaptotagmin-1-SNARE complex in solution. Nature Structural \& Molecular Biology.

Brown, D. A., \& Rose, J. K. (1992). Sorting of GPI-anchored proteins to glycolipid-enriched membrane subdomains during transport to the apical cell surface. Cell, 68(3), 533-544.

Carvalho, K., Ramos, L., Roy, C., \& Picart, C. (2008). Giant unilamellar vesicles containing phosphatidylinositol $(4,5)$ bisphosphate: characterization and functionality. Biophysical Journal, 95(9), 4348-4360.

Chamberlain, L. H., \& Gould, G. W. (2002). The vesicle- and target-SNARE proteins that mediate Glut4 vesicle fusion are localized in detergentinsoluble lipid rafts present on distinct intracellular membranes. Journal of Biological Chemistry, 277(51), 49750-49754.

Chamberlain, L. H., Burgoyne, R. D., \& Gould, G. W. (2001). SNARE proteins are highly enriched in lipid rafts in PC12 cells: Implications for the spatial control of exocytosis. Proceedings of the National Academy of Sciences of the United States of America, 98(10), 5619-5624.

Chapman, E. R. (2008). How does synaptotagmin trigger neurotransmitter release? Annual Review of Biochemistry, 77, 615-641.

Charras, G. T., Yarrow, J. C., Horton, M. A., Mahadevan, L., \& Mitchison, T. J. (2005). Non-equilibration of hydrostatic pressure in blebbing cells. Nature, 435(7040), 365-369.

Cheadle, L., \& Biederer, T. (2012). The novel synaptogenic protein Farp1 links postsynaptic cytoskeletal dynamics and transsynaptic organization. The Journal of Cell Biology, 199(6), 985-1001.

Chintagari, N. R., Jin, N., Wang, P., Narasaraju, T. A., Chen, J., \& Liu, L. (2006). Effect of cholesterol depletion on exocytosis of alveolar type II cells. American Journal of Respiratory Cell and Molecular Biology, 34(6), 677687.

Cicuta, P., Keller, S. L., \& Veatch, S. L. (2007). Diffusion of liquid domains in lipid bilayer membranes. The Journal of Physical Chemistry B, 111(13), 3328.

Clark, E. A., \& Brugge, J. S. (1995). Integrins and signal transduction pathways: the road taken. Science, 268(5208), 233-239.

Cline, H. (2005). Synaptogenesis: a balancing act between excitation and inhibition. Current Biology, 15(6), R203-5.

Collins, B. M., McCoy, A. J., Kent, H. M., Evans, P. R., \& Owen, D. J. (2002). Molecular architecture and functional model of the endocytic AP2 complex. Cell, 109(4), 523-535. 
Cooke, I. R., \& Deserno, M. (2006). Coupling between Lipid Shape and Membrane Curvature. Biophysical Journal, 91(2), 487-495.

Daleke, D. L. (2008). Regulation of phospholipid asymmetry in the erythrocyte membrane. Current Opinion in Hematology, 15(3), 191-195.

Danielli, J. F., \& Davson, H. (1935). A contribution to the theory of permeability of thin films. Journal of Cellular and Comparative Physiology, 5(4), 495-508.

de Almeida, R. F. M., Fedorov, A., \& Prieto, M. (2003).

Sphingomyelin/phosphatidylcholine/cholesterol phase diagram: boundaries and composition of lipid rafts. Biophysical Journal, 85(4), 2406.

de Almeida, R., Loura, L., Prieto, M., Watts, A., Fedorov, A., \& Barrantes, F. J. (2004). Cholesterol modulates the organization of the gamma M4 transmembrane domain of the muscle nicotinic acetylcholine receptor. Biophysical Journal, 86(4), 2261-2272.

de Bartolomeis, A., \& Tomasetti, C. (2012). Calcium-dependent networks in dopamine-glutamate interaction: the role of postsynaptic scaffolding proteins. Molecular Neurobiology, 46(2), 275-296.

de Planque, M. R., Greathouse, D. V., Koeppe, R. E., Schäfer, H., Marsh, D., \& Killian, J. A. (1998). Influence of lipid/peptide hydrophobic mismatch on the thickness of diacylphosphatidylcholine bilayers. A 2H NMR and ESR study using designed transmembrane alpha-helical peptides and gramicidin A. Biochemistry, 37(26), 9333-9345.

de Wit, H., Walter, A. M., Milosevic, I., Gulyas-Kovacs, A., Riedel, D., Sørensen, J. B., \& Verhage, M. (2009). Synaptotagmin-1 docks secretory vesicles to syntaxin-1/SNAP-25 acceptor complexes. Cell, 138(5), 935-946.

Dean, C., \& Dresbach, T. (2006). Neuroligins and neurexins: linking cell adhesion, synapse formation and cognitive function. Trends in Neurosciences, 29(1), 21-29.

Di Paolo, G., \& De Camilli, P. (2006). Phosphoinositides in cell regulation and membrane dynamics. Nature, 443(7112), 651-657.

Di Paolo, G., Moskowitz, H. S., Gipson, K., Wenk, M. R., Voronov, S., Obayashi, M., et al. (2004). Impaired PtdIns(4,5)P2 synthesis in nerve terminals produces defects in synaptic vesicle trafficking. Nature, 431(7007), 415-422.

Dietrich, C., Yang, B., Fujiwara, T., Kusumi, A., \& Jacobson, K. (2002). Relationship of lipid rafts to transient confinement zones detected by single particle tracking. Biophysical Journal, 82(1 Pt 1), 274-284.

Dittman, J., \& Ryan, T. A. (2009). Molecular circuitry of endocytosis at nerve terminals. Annual Review of Cell and Developmental Biology, 25, 133-160. 
Do Heo, W., Inoue, T., Park, W. S., Kim, M. L., Park, B. O., Wandless, T. J., \& Meyer, T. (2006). PI(3,4,5) $\mathrm{P}_{3}$ and $\mathrm{PI}(4,5) \mathrm{P}_{2}$ lipids target proteins with polybasic clusters to the plasma membrane. Science, 314(5804), 1458-1461.

Doeven, M. K., Folgering, J. H. A., Krasnikov, V., Geertsma, E. R., van den Bogaart, G., \& Poolman, B. (2005). Distribution, lateral mobility and function of membrane proteins incorporated into giant unilamellar vesicles. Biophysical Journal, 88(2), 1134-1142.

Dupuy, A. D., \& Engelman, D. M. (2008). Protein area occupancy at the center of the red blood cell membrane. Proceedings of the National Academy of Sciences of the United States of America, 105(8), 2848-2852.

Eggeling, C., Ringemann, C., Medda, R., Schwarzmann, G., Sandhoff, K., Polyakova, S., Belov, V.N., Hein, B., von Middendorff, C., Schönle, A., Hell, S.W. (2009) Direct observation of the nanoscale dynamics of membrane lipids in a living cell. Nature, 457(7233), 1159-1162.

Eggeling, C., Willig, K. I., Sahl, S. J., Hell, S.W. (2015) Lens-based fluorescence nanoscopy. Quarterly Reviews of Biophysics, 48(2), 178-243.

El-Husseini, A. E., Schnell, E., Chetkovich, D. M., Nicoll, R. A., \& Bredt, D. S. (2000). PSD-95 involvement in maturation of excitatory synapses. Science, 290(5495), 1364-1368.

Ellenbroek, W. G., Wang, Y.-H., Christian, D. A., Discher, D. E., Janmey, P. A., \& Liu, A. J. (2011). Divalent cation-dependent formation of electrostatic PIP2 clusters in lipid monolayers. Biophysical Journal, 101(9), 2178-2184.

Fadeel, B., \& Xue, D. (2009). The ins and outs of phospholipid asymmetry in the plasma membrane: roles in health and disease. Critical Reviews in Biochemistry and Molecular Biology, 44(5), 264-277.

Faiss, S., Schuy, S., Weiskopf, D., Steinem, C., Janshoff, A. (2007). Phase transition of individually addressable microstructured membranes visualized by imaging ellipsometry. The Journal of Physical Chemistry B 111, 13979-13986.

Fasshauer, D., \& Margittai, M. (2004). A transient N-terminal interaction of SNAP-25 and syntaxin nucleates SNARE assembly. Journal of Biological Chemistry, 279(9), 7613-7621.

Fdez, E., Martínez-Salvador, M., Beard, M., Woodman, P., \& Hilfiker, S. (2010). Transmembrane-domain determinants for SNARE-mediated membrane fusion. Journal of Cell Science, 123(Pt 14), 2473-2480.

Fehon, R. G., McClatchey, A. I., \& Bretscher, A. (2010). Organizing the cell cortex: the role of ERM proteins. Nature Reviews Molecular Cell Biology, 11(4), 276-287.

Feller, S. E. (2008). Acyl chain conformations in phospholipid bilayers: a 
comparative study of docosahexaenoic acid and saturated fatty acids. Chemistry and Physics of Lipids, 153(1), 76-80.

Fernandez, I., Araç, D., Ubach, J., Gerber, S. H., Shin, O., Gao, Y., et al. (2001). Three-dimensional structure of the synaptotagmin 1 C2B-domain: synaptotagmin 1 as a phospholipid binding machine. Neuron, 32(6), 10571069.

Fernández-Busnadiego, R., Asano, S., Oprisoreanu, A.-M., Sakata, E., Doengi, M., Kochovski, Z., et al. (2013). Cryo-electron tomography reveals a critical role of RIM1 $\alpha$ in synaptic vesicle tethering. The Journal of Cell Biology, 201(5), 725-740.

Fernández-Busnadiego, R., Zuber, B., Maurer, U. E., Cyrklaff, M., Baumeister, W., \& Lucic, V. (2010). Quantitative analysis of the native presynaptic cytomatrix by cryoelectron tomography. The Journal of Cell Biology, 188(1), 145-156.

Förster, T. (1948) Zwischenmolekulare Energiewanderung und Fluorescenz. Ann. Phys. 2, 57-75.

Frolov, V. A., Shnyrova, A. V., \& Zimmerberg, J. (2011). Lipid Polymorphisms and Membrane Shape. Cold Spring Harbor Perspectives in Biology, 3(11), a004747.

Frye, L. D., \& Edidin, M. (1970). The rapid intermixing of cell surface antigens after formation of mouse-human heterokaryons. Journal of Cell Science, $7(2), 319-335$.

Fujiwara, T., Ritchie, K., Murakoshi, H., Jacobson, K., \& Kusumi, A. (2002). Phospholipids undergo hop diffusion in compartmentalized cell membrane. The Journal of Cell Biology, 157(6), 1071-1081.

Fukata, Y., \& Fukata, M. (2010). Protein palmitoylation in neuronal development and synaptic plasticity. Nature Reviews Neuroscience, 11(3), 161-175.

Futai, K., Kim, M. J., Hashikawa, T., Scheiffele, P., Sheng, M., \& Hayashi, Y. (2007). Retrograde modulation of presynaptic release probability through signaling mediated by PSD-95-neuroligin. Nature Neuroscience, 10(2), 186.

Gandasi, N. R., \& Barg, S. (2014). Contact-induced clustering of syntaxin and munc18 docks secretory granules at the exocytosis site. Nature Communications, 5, 3914.

Gandhi, S. P., \& Stevens, C. F. (2003). Three modes of synaptic vesicular recycling revealed by single-vesicle imaging. Nature, 423(6940), 607-613.

Gilmore, A. P., \& Burridge, K. (1996). Regulation of vinculin binding to talin and actin by phosphatidyl-inositol-4-5-bisphosphate. Nature, 381(6582), 531-535. 
Giordano, F., Saheki, Y., Idevall-Hagren, O., Colombo, S. F., Pirruccello, M., Milosevic, I., et al. (2013). PI(4,5)P(2)-dependent and Ca(2+)-regulated ERPM interactions mediated by the extended synaptotagmins. Cell, 153(7), 1494-1509.

Glyvuk, N., Tsytsyura, Y., Geumann, C., D'Hooge, R., Hüve, J., Kratzke, M., et al. (2010). AP-1/sigma1B-adaptin mediates endosomal synaptic vesicle recycling, learning and memory. The EMBO Journal, 29(8), 1318-1330.

Gorter, E., \& Grendel, F. (1925). On bimolecular layers of lipoids on the chromocytes of the blood. The Journal of Experimental Medicine, 41(4), 439.

Göttfert, F., Wurm, C. A., Mueller, V., Berning, S., Cordes, V. C., Honigmann, A., \& Hell, S. W. (2013). Coaligned dual-channel STED nanoscopy and molecular diffusion analysis at $20 \mathrm{~nm}$ resolution. Biophysical Journal, 105(1), L01-L03.

Granseth, B., Odermatt, B., Royle, S. J., \& Lagnado, L. (2006). Clathrinmediated endocytosis is the dominant mechanism of vesicle retrieval at hippocampal synapses. Neuron, 51(6), 773-786.

Greene, L.A., Tischler, A.S. (1976). Establishment of a noradrenergic clonal line of rat adrenal pheocromocytoma cells which respond to nerve growth factor. Proceedings of the National Academy of Sciences of the United States of America, 73(7), 2424-2428.

Grote, E., Baba, M., Ohsumi, Y., \& Novick, P. J. (2000). Geranylgeranylated SNAREs are dominant inhibitors of membrane fusion. The Journal of Cell Biology, 151(2), 453-466.

Gurtovenko, A. A., \& Vattulainen, I. (2007). Molecular Mechanism for Lipid Flip-Flops. The Journal of Physical Chemistry B, 111(48), 13554-13559.

Halemani, N. D., Bethani, I., Rizzoli, S. O., \& Lang, T. (2010). Structure and dynamics of a two-helix SNARE complex in live cells. Traffic, 11(3), 394.

Han, X., Wang, C.-T., Bai, J., Chapman, E. R., \& Jackson, M. B. (2004). Transmembrane segments of syntaxin line the fusion pore of $\mathrm{Ca}^{2+}$ triggered exocytosis. Science, 304(5668), 289-292.

Harlow, M. L., Ress, D., Stoschek, A., Marshall, R. M., \& McMahan, U. J. (2001). The architecture of active zone material at the frog's neuromuscular junction. Nature, 409(6819), 479-484.

Haucke, V., Neher, E., \& Sigrist, S. J. (2011). Protein scaffolds in the coupling of synaptic exocytosis and endocytosis. Nature Reviews Neuroscience, 12(3),

Hay, J. C., \& Martin, T. F. (1993). Phosphatidylinositol transfer protein required for ATP-dependent priming of $\mathrm{Ca}^{2+}$-activated secretion. Nature, 366(6455), 572-575.

Heerklotz, H., \& Seelig, J. (2002). Application of pressure perturbation 
calorimetry to lipid bilayers. Biophysical Journal, 82(3), 1445-1452.

Heijne, von, G. (2006). Membrane-protein topology. Nature Reviews Molecular Cell Biology, 7(12), 909-918.

Hell, S. W. (2003). Toward fluorescence nanoscopy. Nature Biotechnology, 21(11), 1347-1355.

Hell, S. W. (2007). Far-field optical nanoscopy. Science, 316(5828), 1153-1158.

Hell, S. W., \& Kroug, M. (1995). Ground-state-depletion fluorscence microscopy: A concept for breaking the diffraction resolution limit. Applied Physics B, 60(5), 495-497.

Hell, S. W., \& Wichmann, J. (1994). Breaking the diffraction resolution limit by stimulated emission: stimulated-emission-depletion fluorescence microscopy. Optics Letters, 19(11), 780-782.

Hernandez, J. M., Kreutzberger, A. J. B., Kiessling, V., Tamm, L. K., \& Jahn, R. (2014). Variable cooperativity in SNARE-mediated membrane fusion. Proceedings of the National Academy of Sciences of the United States of America, 111(33), 12037-12042.

Herrick, D. Z., Sterbling, S., Rasch, K. A., Hinderliter, A., \& Cafiso, D. S. (2006). Position of synaptotagmin I at the membrane interface: cooperative interactions of tandem C2 domains. Biochemistry, 45(32), 9668-9674.

Herring, B. E., McMillan, K., Pike, C. M., Marks, J., Fox, A. P., \& Xie, Z. (2011). Etomidate and propofol inhibit the neurotransmitter release machinery at different sites. The Journal of Physiology, 589(Pt 5), 1103-1115.

Herring, B. E., Xie, Z., Marks, J., \& Fox, A. P. (2009). Isoflurane inhibits the neurotransmitter release machinery. Journal of Neurophysiology, 102(2), 1265-1273.

Hess, P., Metzger, P., \& Weingart, R. (1982). Free magnesium in sheep, ferret and frog striated muscle at rest measured with ion-selective microelectrodes. The Journal of Physiology, 333, 173-188.

Heumann, R., Kachel, V., \& Thoenen, H. (1983). Relationship between NGFmediated volume increase and "priming effect" in fast and slow reacting clones of PC12 pheochromocytoma cells. Experimental Cell Research, 145(1), 179-190.

Heuser, J. E., \& Reese, T. S. (1973). Evidence for recycling of synaptic vesicle membrane during transmitter release at the frog neuromuscular junction. The Journal of Cell Biology, 57(2), 315-344.

Hofmann, M., Eggeling, C., Jakobs, S., \& Hell, S. W. (2005). Breaking the diffraction barrier in fluorescence microscopy at low light intensities by using reversibly photoswitchable proteins. Proceedings of the National Academy of Sciences of the United States of America, 102(49), 17565-17569. 
Hong, W., \& Lev, S. (2014). Tethering the assembly of SNARE complexes. Trends in Cell Biology, 24(1), 35-43.

Honigmann, A., van den Bogaart, G., Iraheta, E., Risselada, H. J., Milovanovic, D., Mueller, V., et al. (2013). Phosphatidylinositol 4,5-bisphosphate clusters act as molecular beacons for vesicle recruitment. Nature Structural $\mathcal{E}$ Molecular Biology, 20(6), 679-686.

Hoopmann, P., Punge, A., Barysch, S. V., Westphal, V., Bückers, J., Opazo, F., et al. (2010). Endosomal sorting of readily releasable synaptic vesicles. Proceedings of the National Academy of Sciences of the United States of America, 107(44), 19055-19060.

Hua, Y., Sinha, R., Thiel, C. S., Schmidt, R., Hüve, J., Martens, H., et al. (2011). A readily retrievable pool of synaptic vesicles. Nature Neuroscience, 14(7), 833-839.

Imig, C., Min, S.-W., Krinner, S., Arancillo, M., Rosenmund, C., Südhof, T. C., et al. (2014). The morphological and molecular nature of synaptic vesicle priming at presynaptic active zones. Neuron, 84(2), 416-431.

Jacobson, K., Hou, Y., Derzko, Z., Wojcieszyn, J., \& Organisciak, D. (1981). Lipid lateral diffusion in the surface membrane of cells and in multibilayers formed from plasma membrane lipids. Biochemistry, 20(18), 5268-5275.

Jahn, R., \& Fasshauer, D. (2012). Molecular machines governing exocytosis of synaptic vesicles. Nature, 490(7419), 201-207.

Jahn, R., \& Scheller, R. H. (2006). SNAREs - engines for membrane fusion. Nature Reviews Molecular Cell Biology, 7(9), 631-643.

James, D. J., Khodthong, C., Kowalchyk, J. A., \& Martin, T. F. J. (2008). Phosphatidylinositol 4,5-bisphosphate regulates SNARE-dependent membrane fusion. The Journal of Cell Biology, 182(2), 355-366.

Jesús Sot, M Isabel Collado, José L R Arrondo, Alicia Alonso, A., \& Goñi, F. M. (2002). Triton X-100-resistant bilayers: effect of lipid composition and relevance to the raft phenomenon. Langmuir.

Jewell, J. L., Luo, W., Oh, E., Wang, Z., \& Thurmond, D. C. (2008).

Filamentous actin regulates insulin exocytosis through direct interaction with syntaxin 4. Journal of Biological Chemistry, 283(16), 10716-10726.

Johannsson, A., Keightley, C. A., Smith, G. A., Richards, C. D., Hesketh, T. R., \& Metcalfe, J. C. (1981a). The effect of bilayer thickness and n-alkanes on the activity of the $\left(\mathrm{Ca}^{2+}+\mathrm{Mg}^{2+}\right)$-dependent ATPase of sarcoplasmic reticulum. Journal of Biological Chemistry, 256(4), 1643-1650.

Johannsson, A., Smith, G. A., \& Metcalfe, J. C. (1981b). The effect of bilayer thickness on the activity of $\left(\mathrm{Na}^{+}+\mathrm{K}^{+}\right)$-ATPase. Biochimica $\mathcal{E}$ Biophysica 
Acta, 641(2), 416-421.

Jung, N., \& Haucke, V. (2007). Clathrin-mediated endocytosis at synapses. Traffic, 8(9), 1129-1136.

Jung, N., Wienisch, M., Gu, M., Rand, J. B., Müller, S. L., Krause, G., et al. (2007). Molecular basis of synaptic vesicle cargo recognition by the endocytic sorting adaptor stonin 2. The Journal of Cell Biology, 179(7), 14971510.

Kang, R., Wan, J., Arstikaitis, P., Takahashi, H., Huang, K., Bailey, A. O., et al. (2008). Neural palmitoyl-proteomics reveals dynamic synaptic palmitoylation. Nature, 456(7224), 904-909.

Kay, J. G., Murray, R. Z., Pagan, J. K., \& Stow, J. L. (2006). Cytokine secretion via cholesterol-rich lipid raft-associated SNAREs at the phagocytic cup. Journal of Biological Chemistry, 281(17), 11949-11954.

Khuong, T. M., Habets, R. L. P., Kuenen, S., Witkowska, A., Kasprowicz, J., Swerts, J., et al. (2013). Synaptic PI(3,4,5)P3 is required for Syntaxin1A clustering and neurotransmitter release. Neuron, 77(6), 1097-1108.

Killian, J. A., \& Nyholm, T. K. (2006). Peptides in lipid bilayers: the power of simple models. Current Opinion in Structural Biology, 16(4), 473-479.

Kirchhausen, T. (2012). Bending membranes. Nature Cell Biology, 14(9), 906908.

Klar, T. A., Jakobs, S., Dyba, M., Egner, A., \& Hell, S. W. (2000). Fluorescence microscopy with diffraction resolution barrier broken by stimulated emission. Proceedings of the National Academy of Sciences of the United States of America, 97(15), 8206-8210.

Knowles, M. K., Barg, S., Wan, L., Midorikawa, M., Chen, X., \& Almers, W. (2010). Single secretory granules of live cells recruit syntaxin-1 and synaptosomal associated protein 25 (SNAP-25) in large copy numbers. Proceedings of the National Academy of Sciences of the United States of America, 107(48), 20810-20815.

Kolmakov, K., Belov, V. N., Bierwagen, J., Ringemann, C., Müller, V., Eggeling, C., \& Hell, S. W. (2010). Red-emitting rhodamine dyes for fluorescence microscopy and nanoscopy. Chemistry: a European Journal, 16(1), 158-166.

Komura, S., Shirotori, H., Olmsted, P. D., \& Andelman, D. (2004). Lateral phase separation in mixtures of lipids and cholesterol. Europhysics Letters, $67(2), 321$.

Kozlov, M. M., Campelo, F., Liska, N., Chernomordik, L. V., Marrink, S. J., \& McMahon, H. T. (2014). Mechanisms shaping cell membranes. Current Opinion in Cell Biology, 29C, 53-60. 
Krause, M. R., Daly, T. A., Almeida, P. F., \& Regen, S. L. (2014). Push-pull mechanism for lipid raft formation. Langmuir.

Kurps, J., \& de Wit, H. (2012). The role of Munc18-1 and its orthologs in modulation of cortical F-actin in chromaffin cells. Journal of Molecular Neuroscience, 48(2), 339-346.

Kusumi, A., \& Hyde, J. S. (1982). Spin-label saturation-transfer electron spin resonance detection of transient association of rhodopsin in reconstituted membranes. Biochemistry, 21(23), 5978-5983.

Kusumi, A., Suzuki, K. G. N., Kasai, R. S., Ritchie, K., \& Fujiwara, T. K. (2011). Hierarchical mesoscale domain organization of the plasma membrane. Trends in Biochemical Sciences, 36(11), 604-615.

Tamm, L.K., McConnell, M. H. (1985). Supported phospholipid bilayers. Biophysical Journal, 47(1), 105-113.

Laage, R., Rohde, J., Brosig, B., \& Langosch, D. (2000). A conserved membrane-spanning amino acid motif drives homomeric and supports heteromeric assembly of presynaptic SNARE proteins. Journal of Biological Chemistry, 275(23), 17481-17487.

Lafont, F., Verkade, P., Galli, T., Wimmer, C., Louvard, D., \& Simons, K. (1999). Raft association of SNAP receptors acting in apical trafficking in Madin-Darby canine kidney cells. Proceedings of the National Academy of Sciences of the United States of America, 96(7), 3734-3738.

Lakowicz, J. R. (2006). Principles of Fluorescence Spectroscopy. $3^{\text {rd }}$ Edition. Springer US.

Lang, T. (2007). SNARE proteins and "membrane rafts." The Journal of Physiology, 585(Pt 3), 693-698.

Lang, T., Bruns, D., Wenzel, D., Riedel, D., Holroyd, P., Thiele, C., \& Jahn, R. (2001). SNAREs are concentrated in cholesterol-dependent clusters that define docking and fusion sites for exocytosis. The EMBO Journal, 20(9), 2202-2213.

Langmuir, I. (1917). The constitution and fundamental properties of solids and liquids. Journal of the American Chemical Society, 39(9), 1848-1906.

Laux, T., Fukami, K., Thelen, M., Golub, T., Frey, D., \& Caroni, P. (2000). GAP43, MARCKS, and CAP23 modulate PI $(4,5) \mathrm{P}_{2}$ at plasmalemmal rafts, and regulate cell cortex actin dynamics through a common mechanism. The Journal of Cell Biology, 149(7), 1455-1472.

Lenard, J., \& Singer, S. J. (1966). Protein conformation in cell membrane preparations as studied by optical rotatory dispersion and circular dichroism. Proceedings of the National Academy of Sciences of the United States of America, 56(6), 1828-1835. 
Levental, I., Christian, D. A., Wang, Y.-H., Madara, J. J., Discher, D. E., \& Janmey, P. A. (2009). Calcium-dependent lateral organization in phosphatidylinositol 4,5-bisphosphate (PIP2)- and cholesterol-containing monolayers. Biochemistry, 48(34), 8241-8248.

Levental, I., Grzybek, M., \& Simons, K. (2011). Raft domains of variable properties and compositions in plasma membrane vesicles. Proceedings of the National Academy of Sciences of the United States of America, 108(28), 11411-11416.

Levental, I., Lingwood, D., Grzybek, M., Coskun, U., \& Simons, K. (2010). Palmitoylation regulates raft affinity for the majority of integral raft proteins. Proceedings of the National Academy of Sciences of the United States of America, 107(51), 22050-22054.

Li, L. B., Vorobyov, I., \& Allen, T. W. (2012). The role of membrane thickness in charged protein-lipid interactions. Biochimica \& Biophysica Acta (BBA) Biomembranes, 1818(2), 135-145.

Li, S., Eghiaian, F., Sieben, C., Herrmann, A., and Schaap, I. A. T. (2011). Bending and puncturing the influenza lipid envelope. Biophysical Journal 100, 637-645

Lichtenberg, D., Goñi, F. M., \& Heerklotz, H. (2005). Detergent-resistant membranes should not be identified with membrane rafts. Trends in Biochemical Sciences, 30(8), 430-436.

Lin, Q., \& London, E. (2013). Altering hydrophobic sequence lengths shows that hydrophobic mismatch controls affinity for ordered lipid domains (rafts) in the multitransmembrane strand protein perfringolysin O. Journal of Biological Chemistry, 288(2), 1340-1352.

Lindblom, G., Orädd, G., \& Filippov, A. (2006). Lipid lateral diffusion in bilayers with phosphatidylcholine, sphingomyelin and cholesterol. Chemistry and Physics of Lipids, 141(1-2), 179-184.

Lingwood, D., \& Simons, K. (2010). Lipid rafts as a membrane-organizing principle. Science, 327(5961), 46-50.

Lipowsky, R. (1993). Domain-induced budding of fluid membranes. Biophysical Journal, 64(4), 1133-1138.

London, E., \& Brown, D. A. (2000). Insolubility of lipids in triton X-100: physical origin and relationship to sphingolipid/cholesterol membrane domains (rafts). Biochimica \& Biophysica Acta, 1508(1-2), 182-195.

London, R. E. (1991). Methods for measurement of intracellular magnesium: NMR and fluorescence. Annual Review of Physiology, 53, 241-258.

Low, S. H., Vasanji, A., Nanduri, J., He, M., Sharma, N., Koo, M., et al. (2006). Syntaxins 3 and 4 are concentrated in separate clusters on the plasma 
membrane before the establishment of cell polarity. Molecular Biology of the Cell, 17(2), 977-989.

Ma, C., Su, L., Seven, A. B., Xu, Y., \& Rizo, J. (2013). Reconstitution of the vital functions of Munc18 and Munc13 in neurotransmitter release. Science, 339(6118), 421-425.

Magde, D., Elson, E. L., \& Webb, W. W. (1974). Fluorescence correlation spectroscopy. II. An experimental realization. Biopolymers, 13(1), 29-61.

Manford, A. G., Stefan, C. J., Yuan, H. L., Macgurn, J. A., \& Emr, S. D. (2012). ER-to-plasma membrane tethering proteins regulate cell signaling and ER morphology. Developmental Cell, 23(6), 1129-1140.

Margittai, M., Fasshauer, D., Pabst, S., Jahn, R., \& Langen, R. (2001). Homoand heterooligomeric SNARE complexes studied by site-directed spin labeling. Journal of Biological Chemistry, 276(16), 13169-13177.

Marsh, D., \& Smith, I. C. P. (1973). An interacting spin label study of the fluidizing and condensing effects of cholesterol on lecithin bilayers. Biochimica E Biophysica Acta (BBA) - Biomembranes, 298(2), 133-144.

Maycox, P. R., Link, E., Reetz, A., Morris, S. A., \& Jahn, R. (1992). Clathrincoated vesicles in nervous tissue are involved primarily in synaptic vesicle recycling. The Journal of Cell Biology, 118(6), 1379-1388.

McConnell, H. M., Tamm, L. K., \& Weis, R. M. (1984). Periodic structures in lipid monolayer phase transitions. Proceedings of the National Academy of Sciences of the United States of America, 81(10), 3249-3253.

McLaughlin, S., \& Murray, D. (2005). Plasma membrane phosphoinositide organization by protein electrostatics. Nature, 438(7068), 605-611.

McLaughlin, S., Wang, J., Gambhir, A., \& Murray, D. (2002). PIP2 and proteins: interactions, organization adn information flow. Annual Review of Biophysics and Biomolecular Structure, 31(1), 151-175.

Mennicke, U., \& Salditt, T. (2002). Preparation of solid-supported lipid bilayers by spin-coating. Langmuir.

Mesmin, B., Antonny, B., \& Drin, G. (2013). Insights into the mechanisms of sterol transport between organelles. Cellular and Molecular Life Sciences : CMLS, 70(18), 3405-3421.

Milosevic, I., Sørensen, J. B., Lang, T., Krauss, M., Nagy, G., Haucke, V., et al. (2005). Plasmalemmal phosphatidylinositol-4,5-bisphosphate level regulates the releasable vesicle pool size in chromaffin cells. The Journal of Neuroscience, 25(10), 2557-2565.

Milovanovic, D., \& Jahn, R. (2015). Organization and dynamics of SNARE proteins in the presynaptic membrane. Frontiers in Physiology, 6, 89.

Milovanovic, D., Honigmann, A., Koike, S., Göttfert, F., Pähler, G., Junius, M., 
et al. (2015). Hydrophobic mismatch sorts SNARE proteins into distinct membrane domains. Nature Communications, 6, 5984.

Mim, C., \& Unger, V. M. (2012). Membrane curvature and its generation by BAR proteins. Trends in Biochemical Sciences, 37(12), 526-533.

Mitra, K., Ubarretxena-Belandia, I., Taguchi, T., Warren, G., \& Engelman, D. M. (2004). Modulation of the bilayer thickness of exocytic pathway membranes by membrane proteins rather than cholesterol. Proceedings of the National Academy of Sciences of the United States of America, 101(12), 4083-4088.

Mohrmann, R., de Wit, H., Verhage, M., Neher, E., \& Sørensen, J. B. (2010). Fast vesicle fusion in living cells requires at least three SNARE complexes. Science, 330(6003), 502-505.

Morone, N., Fujiwara, T., Murase, K., Kasai, R. S., Ike, H., Yuasa, S., et al. (2006). Three-dimensional reconstruction of the membrane skeleton at the plasma membrane interface by electron tomography. The Journal of Cell Biology, 174(6), 851-862.

Mouritsen, O. G., \& Bloom, M. (1984). Mattress model of lipid-protein interactions in membranes. Biophysical Journal, 46(2), 141-153.

Mueller, V., Ringemann, C., Honigmann, A., Schwarzmann, G., Medda, R., Leutenegger, M., et al. (2011). STED nanoscopy reveals molecular details of cholesterol- and cytoskeleton-modulated lipid interactions in living cells. Biophysical Journal, 101(7), 1651-1660.

Munro, S. (1991). Sequences within and adjacent to the transmembrane segment of alpha-2,6-sialyltransferase specify Golgi retention. The EMBO Journal, 10(12), 3577-3588.

Munro, S. (1995a). A comparison of the transmembrane domains of golgi and plasma membrane proteins. Biochemical Society Transactions, 23(3), 527-30.

Munro, S. (1995b). An investigation of the role of transmembrane domains in Golgi protein retention. The EMBO Journal.

Murray, D. H., \& Tamm, L. K. (2009). Clustering of syntaxin-1A in model membranes Is modulated by phosphatidylinositol 4,5-bisphosphate and cholesterol. Biochemistry, 48(21), 4617-4625.

Murray, D. H., \& Tamm, L. K. (2011). Molecular mechanism of cholesteroland polyphosphoinositide-mediated syntaxin clustering. Biochemistry, 50(42), 9014-9022.

Nagle, J. F., \& Tristram-Nagle, S. (2000). Structure of lipid bilayers. Biochimica $\mathcal{E}$ Biophysica Acta, 1469(3), 159-195.

Nakano, M., Nogami, S., Sato, S., Terano, A., \& Shirataki, H. (2001). Interaction of syntaxin with alpha-fodrin, a major component of the 
submembranous cytoskeleton. Biochemical and Biophysical Research Communications, 288(2), 468-475.

Nicolson, G. L. (2014). The Fluid-Mosaic Model of Membrane Structure: still relevant to understanding the structure, function and dynamics of biological membranes after more than 40 years. Biochimica $\mathcal{E}$ Biophysica Acta, 1838(6), 1451-1466.

Niemelä, P. S., Hyvönen, M. T., \& Vattulainen, I. (2006). Influence of chain length and unsaturation on sphingomyelin bilayers. Biophysical Journal, 90(3), 851-863.

Ohara-Imaizumi, M., Nishiwaki, C., Kikuta, T., Kumakura, K., Nakamichi, Y., \& Nagamatsu, S. (2004a). Site of docking and fusion of insulin secretory granules in live MIN6 cells analyzed by TAT-conjugated anti-syntaxin 1 antibody and total internal reflection fluorescence microscopy. Journal of Biological Chemistry, 279(9), 8403-8408.

Ohara-Imaizumi, M., Nishiwaki, C., Nakamichi, Y., Kikuta, T., Nagai, S., \& Nagamatsu, S. (2004b). Correlation of syntaxin-1 and SNAP-25 clusters with docking and fusion of insulin granules analysed by total internal reflection fluorescence microscopy. Diabetologia, 47(12), 2200-2207.

Owen, D. M., Williamson, D., Rentero, C., \& Gaus, K. (2009). Quantitative microscopy: protein dynamics and membrane organisation. Traffic, 10(8), 962-971.

Pantaloni, D., Le Clainche, C., \& Carlier, M. F. (2001). Mechanism of actinbased motility. Science, 292(5521), 1502-1506.

Papadopoulos, T., \& Soykan, T. (2011). The role of collybistin in gephyrin clustering at inhibitory synapses: facts and open questions. Frontiers in Cellular Neuroscience, 5, 11.

Park, Y., Hernandez, J. M., van den Bogaart, G., Ahmed, S., Holt, M., Riedel, D., \& Jahn, R. (2012). Controlling synaptotagmin activity by electrostatic screening. Nature Structural \& Molecular Biology, 19(10), 991-997.

Petrache, H. I., Zuckerman, D. M., Sachs, J. N., Killian, J. A., Koeppe, R. E., \& Woolf, T. B. (2002). Hydrophobic matching mechanism investigated by molecular dynamics simulations. Langmuir, 18(4), 1340-1351.

Petrov, E. P., \& Schwille, P. (2008). Translational diffusion in lipid membranes beyond the Saffman-Delbruck approximation. Biophysical Journal, 94(5), L41-3.

Pike, L. J. (2006). Rafts defined: a report on the Keystone Symposium on Lipid Rafts and Cell Function (Vol. 47, pp. 1597-1598). Presented at the Journal of Lipid Research, American Society for Biochemistry and Molecular Biology. Pockels, A. (1891). Relations between the surface-tension and relative 
contamination of water surfaces. Nature, 43, 437-439.

Pockels, A. (1894). On the spreading of oil upon water. Nature, 50(1288), 223224.

Predescu, S. A., Predescu, D. N., Shimizu, K., Klein, I. K., \& Malik, A. B. (2005). Cholesterol-dependent syntaxin-4 and SNAP-23 clustering regulates caveolar fusion with the endothelial plasma membrane. Journal of Biological Chemistry, 280(44), 37130-37138.

Prescott, G. R., Gorleku, O. A., Greaves, J., \& Chamberlain, L. H. (2009). Palmitoylation of the synaptic vesicle fusion machinery. Journal of Neurochemistry, 110(4), 1135-1149.

Radhakrishnan, A., Stein, A., Jahn, R., \& Fasshauer, D. (2009). The Ca ${ }^{2+}$ affinity of synaptotagmin 1 is markedly increased by a specific interaction of its C2B domain with phosphatidylinositol 4,5-bisphosphate. Journal of Biological Chemistry, 284(38), 25749-25760.

Ramadurai, S., Holt, A., Krasnikov, V., van den Bogaart, G., Killian, J. A., \& Poolman, B. (2009). Lateral diffusion of membrane proteins. Journal of the American Chemical Society, 131(35), 12650-12656.

Rayleigh, L. (1899). The theory of anomalous dispersion. Phil Mag, 48, 151153.

Regalado, M. P., Terry-Lorenzo, R. T., Waites, C. L., Garner, C. C., \& Malenka, R. C. (2006). Transsynaptic signaling by postsynaptic synapse-associated protein 97. The Journal of Neuroscience, 26(8), 2343-2357.

Reich, C., Horton, M. R., Krause, B., Gast, A. P., Rädler, J. O., \& Nickel, B. (2008). Asymmetric structural features in single supported lipid bilayers containing cholesterol and GM1 resolved with synchrotron X-ray reflectivity. Biophysical Journal, 95(2), 657-668.

Rickman, C., Hu, K., Carroll, J., \& Davletov, B. (2005). Self-assembly of SNARE fusion proteins into star-shaped oligomers. Biochemical Journal, 388(Pt 1), 75-79.

Rickman, C., Medine, C. N., Dun, A. R., Moulton, D. J., Mandula, O., Halemani, N. D., et al. (2010). t-SNARE protein conformations patterned by the lipid microenvironment. Journal of Biological Chemistry, 285(18), 13535-13541.

Ries, J., \& Schwille, P. (2012). Fluorescence correlation spectroscopy. BioEssays : News and Reviews in Molecular, Cellular and Developmental Biology, 34(5), 361-368.

Risselada, H. J., \& Grubmüller, H. (2012). How SNARE molecules mediate membrane fusion: recent insights from molecular simulations. Current Opinion in Structural Biology, 22(2), 187-196. 
Risselada, H. J., Bubnis, G., \& Grubmüller, H. (2014). Expansion of the fusion stalk and its implication for biological membrane fusion. Proceedings of the National Academy of Sciences of the United States of America, 111(30), 1104311048.

Rizo, J., \& Südhof, T. C. (2012). The membrane fusion enigma: SNAREs, Sec1/Munc18 proteins, and their accomplices - guilty as charged? Annual Review of Cell and Developmental Biology, 28(1), 279-308.

Rizo, J., Chen, X., \& Araç, D. (2006). Unraveling the mechanisms of synaptotagmin and SNARE function in neurotransmitter release. Trends in Cell Biology, 16(7), 339-350.

Rizzoli, S. O. (2014). Synaptic vesicle recycling: steps and principles. The EMBO Journal, 33(8), 788-822.

Robertson, J. D. (1960). The molecular structure and contact relationships of cell membranes. Progress Biophysics and Biophysical Chemistry, (10), 343418.

Robertson, J. D. (1963). The occurrence of a subunit pattern in the unit membranes of club endings in Mauthner cell synapses in Goldfish brains. The Journal of Cell Biology, 19, 201-221.

Roder, F., Waichman, S., Paterok, D., Schubert, R., Richter, C., Liedberg, B., \& Piehler, J. (2011). Reconstitution of membrane proteins into polymersupported membranes for probing diffusion and interactions by single molecule techniques. Analytical Chemistry, 83(17), 6792-6799.

Rosenmund, C., Sigler, A., Augustin, I., Reim, K., Brose, N., \& Rhee, J.-S. (2002). Differential control of vesicle priming and short-term plasticity by Munc13 isoforms. Neuron, 33(3), 411-424.

Rossetto, O., Pirazzini, M., \& Montecucco, C. (2014). Botulinum neurotoxins: genetic, structural and mechanistic insights. Nature Reviews. Microbiology, 12(8), 535-549.

Roux, A., Cuvelier, D., Nassoy, P., Prost, J., Bassereau, P., \& Goud, B. (2005). Role of curvature and phase transition in lipid sorting and fission of membrane tubules. The EMBO Journal, 24(8), 1537-1545.

Rubenstein, J. L., Smith, B. A., \& McConnell, H. M. (1979). Lateral diffusion in binary mixtures of cholesterol and phosphatidylcholines. Proceedings of the National Academy of Sciences of the United States of America, 76(1), 15-18.

Saffman, P. G., \& Delbrück, M. (1975). Brownian motion in biological membranes. Proceedings of the National Academy of Sciences of the United States of America, 72(8), 3111-3113.

Saka, S. K., Honigmann, A., Eggeling, C., Hell, S. W., Lang, T., \& Rizzoli, S. O. (2014). Multi-protein assemblies underlie the mesoscale organization of 
the plasma membrane. Nature Communications, 5, 4509.

Sakaba, T., Schneggenburger, R., \& Neher, E. (2002). Estimation of quantal parameters at the calyx of Held synapse. Neuroscience Research, 44(4), 343356.

Salaün, C., Gould, G. W., \& Chamberlain, L. H. (2005). Lipid raft association of SNARE proteins regulates exocytosis in PC12 cells. Journal of Biological Chemistry, 280(20), 19449-19453.

Sarmento, M. J., Coutinho, A., Fedorov, A., Prieto, M., \& Fernandes, F. (2014). $\mathrm{Ca}^{2+}$ induces $\mathrm{PI}(4,5) \mathrm{P}_{2}$ clusters on lipid bilayers at physiological $\mathrm{PI}(4,5) \mathrm{P}_{2}$ and $\mathrm{Ca}^{2+}$ concentrations. Biochimica E Biophysica Acta, 1838(3), 822-830.

Saslowsky, D. E., Lawrence, J. C., Henderson, R. M., \& Edwardson, J. M. (2003). Syntaxin is efficiently excluded from sphingomyelin-enriched domains in supported lipid bilayers containing cholesterol. The Journal of Membrane Biology, 194(3), 153-164.

Schaap, I. A. T., Eghiaian, F., Georges, des, A., and Veigel, C. (2012). Effect of envelope proteins on the mechanical properties of influenza virus. Journal of Biological Chemistry, 287, 41078-41088.

Schindelin, J., Arganda-Carreras, I., Frise, E., Kaynig, V., Longair, M., Pietzsch, T., et al. (2012). Fiji: an open-source platform for biological-image analysis. Nature Methods, 9(7), 676-682.

Schneggenburger, R., \& Neher, E. (2005). Presynaptic calcium and control of vesicle fusion. Current Opinion in Neurobiology, 15(3), 266-274.

Schroeder, R., London, E., \& Brown, D. (1994). Interactions between saturated acyl chains confer detergent resistance on lipids and glycosylphosphatidylinositol (GPI)-anchored proteins: GPI-anchored proteins in liposomes and cells show similar behavior. Proceedings of the National Academy of Sciences of the United States of America, 91(25), 1213012134.

Schuette, C. G., Hatsuzawa, K., Margittai, M., Stein, A., Riedel, D., Kuster, P., et al. (2004). Determinants of liposome fusion mediated by synaptic SNARE proteins. Proceedings of the National Academy of Sciences of the United States of America, 101(9), 2858-2863.

Schulz, T. A., \& Prinz, W. A. (2007). Sterol transport in yeast and the oxysterol binding protein homologue (OSH) family. Biochimica Et Biophysica Acta, Schuy, S., Faiss, S., Yoder, N. C., Kalasani, V., Kumar, K., Janshoff, A., Vogel, R. (2008). Structure and thermotropic phase behavior of fluorinated phospholipid bilayers: a combined attenuated total reflection FTIR spectroscopy and imaging ellipsometry study. Journal of Physical Chemistry B 112, 8250-8256. 
Schwenen LL, Hubrich R, Milovanovic D, Geil B, Yang J, Kros A, Jahn R, Steinem C. Resolving single membrane fusion events on planar porespanning membranes. (2015) Scientific Reports, 5: 12006

Sezgin, E., Kaiser, H.-J., Baumgart, T., Schwille, P., Simons, K., \& Levental, I. (2012). Elucidating membrane structure and protein behavior using giant plasma membrane vesicles. Nature Protocols, 7(6), 1042-1051.

Sharpe, H. J., Stevens, T. J., \& Munro, S. (2010). A comprehensive comparison of transmembrane domains reveals organelle-specific properties. Cell, 142(1), 158-169.

Shi, L., Shen, Q.-T., Kiel, A., Wang, J., Wang, H.-W., Melia, T. J., et al. (2012). SNARE proteins: one to fuse and three to keep the nascent fusion pore open. Science, 335(6074), 1355-1359.

Sieber, J. J., Willig, K. I., Heintzmann, R., Hell, S. W., \& Lang, T. (2006). The SNARE motif is essential for the formation of syntaxin clusters in the plasma membrane. Biophysical Journal, 90(8), 2843-2851.

Sieber, J. J., Willig, K. I., Kutzner, C., Gerding-Reimers, C., Harke, B., Donnert, G., et al. (2007). Anatomy and dynamics of a supramolecular membrane protein cluster. Science, 317(5841), 1072-1076.

Silvius, J. R., del Giudice, D., \& Lafleur, M. (1996). Cholesterol at different bilayer concentrations can promote or antagonize lateral segregation of phospholipids of differing acyl chain length. Biochemistry, 35(48), 15198-

Simons, K., \& Ikonen, E. (1997). Functional rafts in cell membranes. Nature, 387(6633), 569-572.

Simons, K., \& van Meer, G. (1988). Lipid sorting in epithelial cells. Biochemistry, 27(17), 6197-6202.

Singer, S. J., \& Nicolson, G. L. (1972). The fluid mosaic model of the structure of cell membranes. Science, 175(4023), 720-731.

Sinha, R., Ahmed, S., Jahn, R., \& Klingauf, J. (2011). Two synaptobrevin molecules are sufficient for vesicle fusion in central nervous system synapses. Proceedings of the National Academy of Sciences of the United States of America, 108(34), 14318-14323.

Sparr, E., Ganchev, D. N., Snel, M. M. E., Ridder, A. N. J. A., KroonBatenburg, L. M. J., Chupin, V., et al. (2005). Molecular organization in striated domains induced by transmembrane alpha-helical peptides in dipalmitoyl phosphatidylcholine bilayers. Biochemistry, 44(1), 2-10.

Stefan, C. J., Manford, A. G., \& Emr, S. D. (2013). ER-PM connections: sites of information transfer and inter-organelle communication. Current Opinion in Cell Biology, 25(4), 434-442.

Stein, A., Radhakrishnan, A., Riedel, D., Fasshauer, D., \& Jahn, R. (2007). 
Synaptotagmin activates membrane fusion through a $\mathrm{Ca}^{2+}$-dependent trans interaction with phospholipids. Nature Structural $\mathcal{E}$ Molecular Biology, 14(10), 904-911.

Stein, A., Weber, G., Wahl, M. C., \& Jahn, R. (2009). Helical extension of the neuronal SNARE complex into the membrane. Nature, 460(7254), 525-528.

Sutton, R. B., Fasshauer, D., Jahn, R., \& Brunger, A. T. (1998). Crystal structure of a SNARE complex involved in synaptic exocytosis at 2.4 A resolution. Nature, 395(6700), 347-353.

Südhof, T. C. (2004). The synaptic vesicle cycle. Annual Review of Neuroscience, $27,509-547$.

Szule, J. A., Harlow, M. L., Jung, J. H., De-Miguel, F. F., Marshall, R. M., \& McMahan, U. J. (2012). Regulation of synaptic vesicle docking by different classes of macromolecules in active zone material. PLOS ONE, 7(3), e33333.

Takamori, S., Holt, M., Stenius, K., Lemke, E. A., Grønborg, M., Riedel, D., et al. (2006). Molecular anatomy of a trafficking organelle. Cell, 127(4), 831846.

Tang, J., Maximov, A., Shin, O.-H., Dai, H., Rizo, J., \& Südhof, T. C. (2006). A complexin/synaptotagmin 1 switch controls fast synaptic vesicle exocytosis. Cell, 126(6), 1175-1187.

Torregrosa-Hetland, C. J., Villanueva, J., Garcia-Martinez, V., ExpósitoRomero, G., Francés, M. D. M., \& Gutiérrez, L. M. (2013). Cortical F-actin affects the localization and dynamics of SNAP-25 membrane clusters in chromaffin cells. The International Journal of Biochemistry $\mathcal{E}$ Cell Biology, 45(3), 583-592.

Torregrosa-Hetland, C. J., Villanueva, J., Giner, D., López-Font, I., Nadal, A., Quesada, I., et al. (2011). The F-actin cortical network is a major factor influencing the organization of the secretory machinery in chromaffin cells. Journal of Cell Science, 124(Pt 5), 727-734.

van den Bogaart, G., Holt, M. G., Bunt, G., Riedel, D., Wouters, F. S., \& Jahn, R. (2010). One SNARE complex is sufficient for membrane fusion. Nature Structural \& Molecular Biology, 17(3), 358-364.

van den Bogaart, G., Lang, T., \& Jahn, R. (2013). Microdomains of SNARE proteins in the plasma membrane. Current Topics in Membranes, 72, 193230.

van den Bogaart, G., Meyenberg, K., Diederichsen, U., \& Jahn, R. (2012). Phosphatidylinositol 4,5-bisphosphate increases $\mathrm{Ca}^{2+}$ affinity of synaptotagmin-1 by 40-fold. Journal of Biological Chemistry, 287(20), 1644716453.

van den Bogaart, G., Meyenberg, K., Risselada, H. J., Amin, H., Willig, K. I., 
Hubrich, B. E., et al. (2011). Membrane protein sequestering by ionic protein-lipid interactions. Nature, 479(7374), 552-555.

van Meer, G., Voelker, D. R., \& Feigenson, G. W. (2008). Membrane lipids: where they are and how they behave. Nature Reviews Molecular Cell Biology, 9(2), 112-124.

Veatch, S. L., \& Keller, S. L. (2005). Seeing spots: Complex phase behavior in simple membranes. Biochimica E Biophysica Acta (BBA) - Molecular Cell Research, 1746(3), 172-185.

Vennekate, W., Schröder, S., Lin, C.-C., van den Bogaart, G., Grunwald, M., Jahn, R., \& Walla, P. J. (2012). Cis- and trans-membrane interactions of synaptotagmin-1. Proceedings of the National Academy of Sciences of the United States of America, 109(27), 11037-11042.

Verhage, M., Maia, A. S., Plomp, J. J., Brussaard, A. B., Heeroma, J. H., Vermeer, H., et al. (2000). Synaptic assembly of the brain in the absence of neurotransmitter secretion. Science, 287(5454), 864-869.

Villanueva, J., Torres, V., Torregrosa-Hetland, C. J., Garcia-Martinez, V., López-Font, I., Viniegra, S., \& Gutiérrez, L. M. (2012). F-actin-myosin II inhibitors affect chromaffin granule plasma membrane distance and fusion kinetics by retraction of the cytoskeletal cortex. Journal of Molecular Neuroscience, 48(2), 328-338.

Voets, T., Toonen, R. F., Brian, E. C., de Wit, H., Moser, T., Rettig, J., et al. (2001). Munc18-1 promotes large dense-core vesicle docking. Neuron, 31(4), 581-591.

Vukojević, V., Pramanik, A., Yakovleva, T., Rigler, R., Terenius, L., \& Bakalkin, G. (2005). Study of molecular events in cells by fluorescence correlation spectroscopy. Cellular and Molecular Life Sciences : CMLS, 62(5), 535-550.

Wagner, M. L., \& Tamm, L. K. (2001). Reconstituted syntaxin1a/SNAP25 interacts with negatively charged lipids as measured by lateral diffusion in planar supported bilayers. Biophysical Journal, 81(1), 266-275.

Wallach, D. F., \& Zahler, P. H. (1966). Protein conformations in cellular membranes. Proceedings of the National Academy of Sciences of the United States of America, 56(5), 1552-1559.

Wang, Y.-H., Collins, A., Guo, L., Smith-Dupont, K. B., Gai, F., Svitkina, T., \& Janmey, P. A. (2012). Divalent cation-induced cluster formation by polyphosphoinositides in model membranes. Journal of the American Chemical Society, 134(7), 3387-3395.

Wang, Y.-H., Slochower, D. R., \& Janmey, P. A. (2014). Counterion-mediated cluster formation by polyphosphoinositides. Chemistry and Physics of 
Lipids, 1-14.

Watanabe, M., Nomura, K., Ohyama, A., Ishikawa, R., Komiya, Y., Hosaka, K., et al. (2005). Myosin-Va regulates exocytosis through the submicromolar $\mathrm{Ca}^{2+}$-dependent binding of syntaxin-1A. Molecular Biology of the Cell, 16(10), 4519-4530.

Watanabe, S., Lehmann, M., Hujber, E., Fetter, R. D., Richards, J., SöhlKielczynski, B., et al. (2014a). Nanometer-resolution fluorescence electron microscopy (nano-EM) in cultured cells. Methods in Molecular Biology (Clifton, N.J.), 1117, 503-526.

Watanabe, S., Liu, Q., Davis, M. W., Hollopeter, G., Thomas, N., Jorgensen, N. B., \& Jorgensen, E. M. (2013a). Ultrafast endocytosis at Caenorhabditis elegans neuromuscular junctions. eLife, 2, e00723.

Watanabe, S., Rost, B. R., Camacho-Pérez, M., Davis, M. W., Söhl-Kielczynski, B., Rosenmund, C., \& Jorgensen, E. M. (2013b). Ultrafast endocytosis at mouse hippocampal synapses. Nature, 504(7479), 242-247.

Watanabe, S., Trimbuch, T., Camacho-Pérez, M., Rost, B. R., Brokowski, B., Söhl-Kielczynski, B., et al. (2014b). Clathrin regenerates synaptic vesicles from endosomes. Nature.

Watson, R. T., \& Pessin, J. E. (2001). Transmembrane domain length determines intracellular membrane compartment localization of syntaxins 3, 4, and 5. American Journal of Physiology. Cell Physiology, 281(1), C215-23.

Weiß, K., Neef, A., Van, Q., Kramer, S., Gregor, I., \& Enderlein, J. (2013). Quantifying the diffusion of membrane proteins and peptides in black lipid membranes with 2-focus fluorescence correlation spectroscopy. Biophysical Journal, 105(2), 455-462.

Wen, P. J., Osborne, S. L., \& Meunier, F. A. (2011). Dynamic control of neuroexocytosis by phosphoinositides in health and disease. Progress in Lipid Research, 50(1), 52-61.

Wienisch, M., \& Klingauf, J. (2006). Vesicular proteins exocytosed and subsequently retrieved by compensatory endocytosis are nonidentical. Nature Neuroscience, 9(8), 1019-1027.

Wilhelm, B. G., Mandad, S., Truckenbrodt, S., Kröhnert, K., Schäfer, C., Rammner, B., et al. (2014). Composition of isolated synaptic boutons reveals the amounts of vesicle trafficking proteins. Science, 344(6187), 1023-1028.

Willig, K. I., Rizzoli, S. O., Westphal, V., Jahn, R., \& Hell, S. W. (2006). STED microscopy reveals that synaptotagmin remains clustered after synaptic vesicle exocytosis. Nature, 440(7086), 935-939.

Wilson, B. S., Steinberg, S. L., Liederman, K., Pfeiffer, J. R., Surviladze, Z., 
Zhang, J., et al. (2004). Markers for detergent-resistant lipid rafts occupy distinct and dynamic domains in native membranes. Molecular Biology of the Cell, 15(6), 2580-2592.

Wittenmayer, N., Körber, C., Liu, H., Kremer, T., Varoqueaux, F., Chapman, E. R., et al. (2009). Postsynaptic Neuroligin1 regulates presynaptic maturation. Proceedings of the National Academy of Sciences of the United States of America, 106(32), 13564-13569.

Woolfrey, K. M., Srivastava, D. P., Photowala, H., Yamashita, M., Barbolina, M. V., Cahill, M. E., et al. (2009). Epac2 induces synapse remodeling and depression and its disease-associated forms alter spines. Nature Neuroscience, 12(10), 1275-1284.

Woronowicz, K., Dilks, J. R., Rozenvayn, N., Dowal, L., Blair, P. S., Peters, C. G., et al. (2010). The platelet actin cytoskeleton associates with SNAREs and participates in $\alpha$-granule secretion. Biochemistry, 49(21), 4533-4542. Xie, Z., McMillan, K., Pike, C. M., Cahill, A. L., Herring, B. E., Wang, Q., \& Fox, A. P. (2013). Interaction of anesthetics with neurotransmitter release machinery proteins. Journal of Neurophysiology, 109(3), 758-767.

Yang, X., Xu, P., Xiao, Y., Xiong, X., \& Xu, T. (2006). Domain Requirement for the Membrane Trafficking and Targeting of Syntaxin 1A. Journal of Biological Chemistry, 281(22), 15457-15463.

Yoshimori, T., Keller, P., Roth, M. G., \& Simons, K. (1996). Different biosynthetic transport routes to the plasma membrane in $\mathrm{BHK}$ and $\mathrm{CHO}$ cells. The Journal of Cell Biology, 133(2), 247-256.

Zaccai, G. (2000). How soft is a protein? A protein dynamics force constant measured by neutron scattering. Science, 288(5471), 1604-1607.

Zaccai, G., Blasie, J. K., \& Schoenborn, B. P. (1975). Neutron diffraction studies on the location of water in lecithin bilayer model membranes. Proceedings of the National Academy of Sciences of the United States of America, 72(1), 376380.

Zhou, P., Bacaj, T., Yang, X., Pang, Z. P., \& Südhof, T. C. (2013). Lipidanchored SNAREs lacking transmembrane regions fully support membrane fusion during neurotransmitter release. Neuron, 80(2), 470-483.

Zilly, F. E., Halemani, N. D., Walrafen, D., Spitta, L., Schreiber, A., Jahn, R., \& Lang, T. (2011). $\mathrm{Ca}^{2+}$ induces clustering of membrane proteins in the plasma membrane via electrostatic interactions. The EMBO Journal, 30(7), 1209-1220.

Zilly, F. E., Sørensen, J. B., Jahn, R., \& Lang, T. (2006). Munc18-bound syntaxin readily forms SNARE complexes with synaptobrevin in native plasma membranes. PLoS Biology, 4(10), e330. 


\section{APPENDICES}

\subsection{Information on peptide synthesis}

Peptides were synthesized by Group of Prof. Ulf Diederichsen (University of Göttingen). Fmoc solid phase synthesis was done on preloaded Fmoc-Gly-Wang resin LL with chain elongation performed on the microwave assisted automatic peptide synthesizer Liberty with an additional module (Discover, CEM Corporation) that provided microwave energy of 2,45 MHz. Deprotection was performed with $20 \%$ piperidine in NMP (N-methyl-2pyrrolidone) and amino acids were prepared as $0.2 \mathrm{M}$ solutions in NMP. Coupling was done with $0.5 \mathrm{M}$ HBTU/HOBt (HBTU-based 1hydroxybenzotriazole) in DMF, 0.2 M amino acids in NMP and $2 \mathrm{M}$ DIPEA (N,N-diisopropylethylamine) in NMP. Deprotection (two steps of 30 and 180 $\mathrm{s}$ respectively), coupling $\left(300 \mathrm{~s}\right.$ at $\left.75^{\circ} \mathrm{C}\right)$ and capping were performed with microwave energy and $\mathrm{N}_{2}$ mixing. The fluorescent dyes Atto647N NHS-ester (Atto-Tec) and Rhodamine Red (Invitrogen) were coupled to the N-terminus to the peptides on solid support using an excess of dye at room temperature for 12 hours. After coupling, the resin was washed with DCM and diethylether and dried in a high vacuum overnight. Cleavage from the solid support was carried with a mixture of TFA: $\mathrm{H}_{2} \mathrm{O}$ :EDT:TES (94:2.5:2.5:10; 10 $\mathrm{ml} / \mathrm{g}$ resin) for 2 hours. Subsequently the resin was filtered off, the solution concentrated and the peptides were precipitated from cold MTBE, centrifuged and lyophilized. Since the fluorophore is coupled to the N-terminal at the very end of synthesis, only complete peptides were fluorescently labeled. All peptides were verified by electrospray ionization mass spectroscopy ESI-MS. 


\subsection{Information on imaging ellipsometry}

Imaging ellipsometry measurements were done by Group of Prof. Andreas Janshoff (University of Göttingen). Lipid stock solutions ( $\left(_{\text {lipid }}=1-10\right.$ $\mathrm{mg} / \mathrm{ml}$ ) were prepared in chloroform and transformed into lipid films by removal of the solvent in a nitrogen stream followed by $3 \mathrm{~h}$ drying in vacuum. Multilamellar vesicles (MLV) were produced by resuspending the lipid films in buffer (50 mM HEPES pH 7.4 with $3 \mathrm{mM} \mathrm{Ca}^{2+}$ ) at a concentration of 1 $\mathrm{mg} / \mathrm{ml}$. MLVs were transformed into small unilamellar vesicles (SUV) by sonication ( $50 \mathrm{~W}, 0.4 \mathrm{~s}$ pulse, $30 \mathrm{~min}$ ) in a vessel resonator (Sonoplus HD 2070). Average vesicle size was $30-50 \mathrm{~nm}$ as determined by dynamic light scattering. Si-Wafers were cleaned in $\mathrm{H}_{2} \mathrm{O}_{2} / \mathrm{NH}_{3} / \mathrm{H}_{2} \mathrm{O} 1: 1: 5$ at $70^{\circ} \mathrm{C}$ for 15 min and afterwards hydrophilized for $1 \mathrm{~min}$ in $\mathrm{O}_{2}$-plasma. For preparing the lipid bilayer, freshly prepared SUVs were spreaded for $10-30 \mathrm{~min}$ on a hydrophilized Si-Wafer at a concentration of $0.2 \mathrm{mg} / \mathrm{ml}$ in $50 \mathrm{mM}$ HEPES $\mathrm{pH} 7.4$ with $3 \mathrm{mM} \mathrm{Ca}^{2+}$. Measurements were carried out in the same buffer in a closed fluid chamber. Ellipsometry experiments were performed using an imaging ellipsometer EP3 3 -SW from Accurion as described previously (Faiss et al., 2007; Schuy et al., 2008). This method offers the possibility to measure thin layer thicknesses in real time within a convective flow at defined temperature. The principal angle del determined by this method is proportional to layer thicknesses for sufficiently thin dielectric layers $(h<30 \mathrm{~nm})$. Absolute height changes resulting from spreaded solid supported membranes (SSM) were computed from the angle del which is linearly related to the height for thin layers $\left(1 \mathrm{~nm} \approx 0.91^{\circ} \mathrm{del}\right)$ and assuming a refractive index of 1.5 for the all used lipids. 


\subsection{Information on atomic force microscopy measurements}

Atomic force microscopy (AFM) measurements were done by Group of Dr. Iwan Schaap (University of Göttingen). The glass coverslips were cleaned using the Plasma cleaner Fempto timer with $40 \mathrm{kHz} / 100 \mathrm{~W}$ generator (Diener electronic, Germany) and a lipid/sx-1 TMD bilayer was generated by spincoating (10 s at 100xg). The reconstituted bilayers were imaged with a Cervantes Full Mode AFM System (Nanotec, Spain) using AC40TS cantilevers $\left(\omega_{0}=110 \mathrm{kHz}, \mathrm{k}=0.1 \mathrm{~N} / \mathrm{m}\right.$; Olympus, Japan) similarly as in (Li et al., 2011, Schaap et al., 2012). Calibration of the cantilevers was accomplished by using the thermal noise spectrum. We employed the jumping mode plus (jump off $100 \mathrm{~nm}$, sample points 50), which allows scanning at controlled vertical forces between $0.2 \mathrm{nN}$ and several $\mathrm{nN}$. 


\section{LIST of FIGURES}

\section{INTRODUCTION}

1.1. Evolving view on the plasma membrane organization .................. 2

1.2. Scheme of the lipid bilayer ........................................ 3

1.3. Schemes and Newman projections of acyl-chains with all bonds in trans

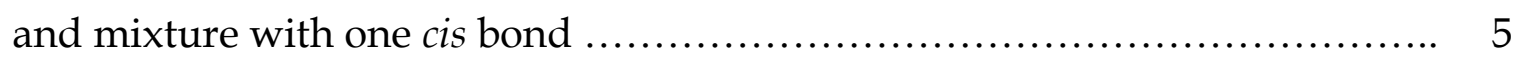

1.4. Formulas of the major lipid classes .................................. 6

1.5. Schematic representation of three characteristic lipid phases ............... 7

1.6. The lateral diffusion coefficient of NBD-PE in DPPC bilayer ............... 8

1.7. Phase transition in model lipid membranes ............................ 9

1.8. Different lipid shapes underlie intrinsic membrane curvature .............. 12

1.9. Different biochemical properties of lipids generate two major membrane territories in cell ....................................................... 14

1.10. Lipid asymmetry in the plasma membrane ........................... 16

1.11. Lipid phases in the plasma membrane ................................ 18

1.12. Picket-fence model of the plasma membrane organization .............. 20

1.13. Organization and coupling of the excitatory pre- and postsynapse ........ 22

1.14. SNARE proteins catalyze membrane fusion ............................ 24

1.15. Effects of hydrophobic mismatch between lipids and proteins.............. 27

1.16. Syntaxin $1 \mathrm{~A}$ interacts with $\mathrm{PI}(4,5) \mathrm{P}_{2}$ in the membrane ...................... 29

1.17. Supramolecular syntaxin clusters reconstituted in silico .................. 30

1.18. Scheme of the synaptic vesicle cycle ................................. 33

1.19. Plasma membrane SNAREs are too short to span the entire membrane .... 36

\section{MATERIALS AND METHODS}

2.1. Scheme of the assay for preparation of polymer supported membranes ... 40

2.2. Scheme of LUVs generation by extrusion ................................ 42

2.3. DLS measurement of the LUVs prepared by extrusion procedure .......... 43

2.4. Preparation of cell sheets .......................................... 44

2.5. Principles of stimulated emission depletion (STED) nanoscopy ............. 49

2.6. Principles of fluorescence correlation spectroscopy ..................... 51 
2.7. Jablonski diagram of the electronic states of donor (left) and acceptor (right) molecules in FRET

\section{RESULTS}

3.1. Hydrophobic mismatch between the transmembrane domain (TMD) of proteins and the surrounding lipid environment ........................ 56

3.2. Hydrophobic mismatch clusters syntaxin 1 TMD in lipid bilayers ....... 57

3.3. Sx-1 TMD and sx-4 TMD clustering determined by FRET assay .......... 58

3.4. Cholesterol increases thickness of the lipid bilayer and further enhances hydrophobic mismatch ............................................. 60

3.5. Visualizing sx-1 TMD clustering in GUVs .......................... 61

3.6. Reconstitution of sx-1 TMD in polymer supported membranes .......... 62

3.7. Specific protein-protein interactions of TMDs do not affect clustering in case of positive and negative mismatch .................................. 63

3.8. Two color STED images of syntaxin 1 and 4 colocalization with the $\mathrm{PI}(4,5) \mathrm{P}_{2}$ in the plasma membrane sheets of PC12 cells ...................... 65

3.9. Cholesterol and $\mathrm{PI}(4,5) \mathrm{P}_{2}$ both induce clustering of syntaxin TMDs ...... 66

3.10. Determining the charge screening effect on sx-1TMD and $\mathrm{PI}(4,5) \mathrm{P}_{2}$

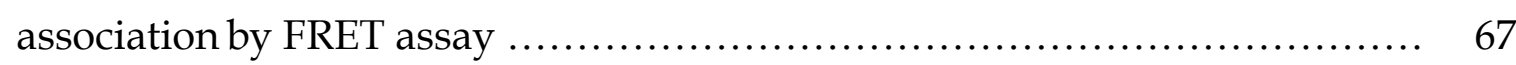

3.11. Two-color STED microscopy of PC12 membrane sheets immunostained against syntaxins 1 (red) and 4 (green) ................................ 68

3.12. Clustering of sx- 1 TMD and sx-4 TMD measured by FRET assay in liposomes composed of either C18:1 PC or an equimolar mixture of C14:1, C16:1, C18:1 and C20:1 PC

3.13. Differences in the length of the TMDs contribute to segregation of syntaxin 1 and 4 into distinct domains in the plasma membrane ............. 70

3.14. Calcium promotes clustering of syntaxin 1 in the plasma membrane of PC12 cells ............................................................. 73

3.15. Calcium promoted syntaxin clustering requires $\mathrm{PI}(4,5) \mathrm{P}_{2} \ldots \ldots \ldots \ldots \ldots . . . \ldots 4$

3.16. Sx-1 TMD/PI $(4,5) \mathrm{P}_{2}$ domains reconstituted in lipid bilayers observed by

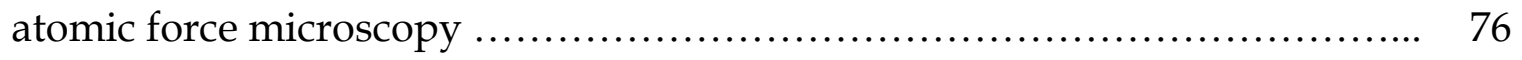

3.17. Reversible clustering of syntaxin $1 / \mathrm{PI}(4,5) \mathrm{P}_{2}$ domains by $\mathrm{Ca}^{2+} \ldots \ldots \ldots \ldots .77$

3.18. $\mathrm{Ca}^{2+}$ induces $\mathrm{PI}(4,5) \mathrm{P}_{2}$ clustering in the lipid independently of sx-1 TMD 78 
3.19. Calcium acts as charge bridge that connects multiple syntaxin $1 / \mathrm{PI}(4,5)_{2}$ complexes

3.20. Effect of the charge screening effect on sx-1 TMD clustering in the absence and presence of $\mathrm{Ca}^{2+}$ measured by FRET

\section{DISCUSSION}

4.1. Synergistic model of syntaxin clustering in the membrane 85

4.2. Model of calcium-induced syntaxin domain formation 88

\section{CONCLUSIONS and PERSPECTIVES}

5.1. Biological membranes are modular systems 


\section{LIST of ABBREVIATIONS}

ABC transporters: ATP-binding cassette family transporters

AFM: atomic force microscope

ALPS: amphipathic lipid packing sensor motifs

AMPAR: $\alpha$-amino-3-hydroxy-5-methyl-4-isoxazolepropionic acid receptor

ATP: adenosine triphosphate

BAR: Bin-Amphiphysin-Rvs domain

C14:1 PC: 1,2-dimyristoleoyl-sn-glycero-3-phosphocholine

C16:1 PC: 1,2-dipalmitoleoyl-sn-glycero-3-phosphocholine

C18:1 PC: 1,2-dioleoyl-sn-glycero-3-phosphocholine

C20:1 PC: 1,2-dieicosenoyl-sn-glycero-3-phosphocholine

CASK: calcium/calmodulin-dependent serine protein kinase

DAPEG: diaminopolyethilene glycol

DCM: dichloromethane

DiO: 3,3'-dilinoleyloxacarbocyanine perchlorate

DIPEA: N,N-diisopropylethylamine

DLS: dynamic light scattering

DMSO: dimethyl sulfoxide

DOPC: dioleoylphosphatidylcholine

DOPE: dioleoylphosphatidylethanolamine

DOPS: dioleoylphosphatidylserine

DTT: dithiothreitol

EDT: 1,2-ethanedithiol

EDTA: ethylenediaminetetraacetic acid

ERM: ezrin, radixin and merlin protein family

ESI-MS: electrospray ionization mass spectrometry

FCS: fluorescence correlation spectroscopy

FRET: Förster resonance energy transfer

GOPTS: (3-glycidyloxypropyl)-trimethoxysilane

GPMV: giant plasma membrane-derived vesicles

GUV: giant unilamellar vesicles

HBTU: 2-(1H-benzotriazol-1-yl)-1,1,3,3-tetramethyluronium hexafluorophosphate

HEPES: 4-(2-hydroxyethyl)-1-piperazineethanesulfonic acid 
HOBt: 1-hydroxybenzotriazole

LUV: large unilamellar vesicles

MTBA: methyl tert-butyl ether

NBD-PE: N-(7-nitrobenz-2-oxa-1,3-Diazol-4-yl)-1,2-Dihexadecanoyl-sn-

glycero-3-phosphoethanolamine

NMDAR: N-methyl-D-aspartate receptor

NMP: N-methyl-2-pyrrolidone

ORP: oxysterol-binding protein (OSBP) related-proteins

PC: phosphatidylcholine

PC12 cells: pheochromocytoma cell line

PDZ: postsynaptic density-95/Discs large/Zona occludens-1

PE: phosphatidylethanolamine

PI4P: phosphatidylinositol 4-phosphate

$\mathrm{PI}(4,5) \mathrm{P}_{2}$ : phosphatidylinositol 4,5-bisphosphate

$\mathrm{PI}(3,4,5) \mathrm{P}_{3}$ : phosphatidylinositol 3,4,5-trisphosphate

PS: phosphatidylserine

PSD95: postsynaptic density protein 95

PSF: point spread function

PSM: polymer supported membranes

RIM: Rab3-interacting molecule

SM: sphingomyelin

SMP: synaptotagmin-like-mitochondrial-lipid binding protein domain

SNARE: soluble N-ethylmalemide sensitive factor-attachment protein receptor

STED: stimulated emission depletion nanoscopy

SUV: small unilamellar vesicles

SSM: solid supported membranes

TES: triethylsilane

TFA: trifluoroacetic acid

TMD: transmembrane domain 


\subsection{CURRICULUM VITAE}

Dragomir Milovanović

Date of birth: 23/10/1988

Nationality: Serbian

Marital status: single

Contact details: Department of Neurobiology

Max Planck Institute for Biophysical Chemistry

Am Fassberg 11

37077 Göttingen, Germany

+49(0)551201 1670 (office)

+49 (0)176 81246601 (mob)

drago.milovanovic@gmail.com

dmilova@gwdg.de

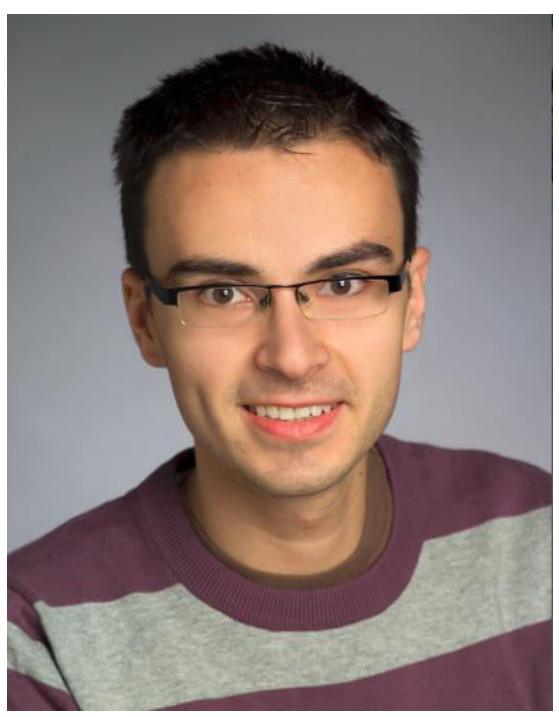

\section{EDUCATION}

2012 - present: Doctoral studies (expected graduation date: October $5^{\text {th }}, 2015$ ) Department of Neurobiology, Max Planck Institute for Biophysical Chemistry and Göttingen Graduate School for Neuroscience, Biophysics and Molecular Biosciences, Georg-August-Universität Göttingen

Title of the Thesis: Nanoscale organization and dynamics of SNARE proteins in the presynaptic membranes

2011 - 2012: graduate studies:

International Max Planck Research School in Molecular Biology and Georg-August-Universität Göttingen;

Masters Examination, August 2012: performance excellent/A

2007 - 2011: B.Sc. (Hons)/Dipl.

Faculty of Chemistry, University of Belgrade, Serbia

Department of Biochemistry : 2007-2008; 2009 - 2010;

Performance: 9.97 (10.0 scale); Top student in the Class

2008 - 2009: US Government-sponsored student fellowship

Department of Biochemistry, College of Natural Sciences and Mathematics, Indiana University of Pennsylvania, USA; performance: 4.0 (4.0 scale) 


\section{INVITED TALKS}

- 2015 - Invited Speaker at the $15^{\text {th }}$ International Membrane Research Forum, Kyoto, Japan

- 2014, 2015 - Invited Speaker at the II \& III Petnica School of Microscopy, Petnica Science Center and University of Belgrade, Serbia

- 2015 - Awarded Student Talk at 12 ${ }^{\text {th }}$ Horizons in Molecular Biology, Göttingen, Germany

- 2014 - Guest Lecturer at the Biochemistry 301 (Biochemistry majors, III year undergraduate course), Indiana University of Pennsylvania, USA.

- 2014 - Chemistry Seminar Series, Invited Speaker, Indiana University of Pennsylvania.

- 2014 - Guest Lecture at the International Science Camp, XLAB, Germany

- 2012 - Selected speaker at the FEBS Advanced Microscopy Course, Wageningen and Nijmegen, the Netherlands.

- 2012 - Selected speaker at the 50th Meeting of the Serbian Chemical Society, Belgrade, Serbia.

\section{AWARDS, FELLOWSHIPS, DISTINCTIONS}

- 2015 Max Planck Society Fellow: participation at the $65^{\text {th }}$ Interdisciplinary Lindau Nobel Laureate Meeting, Lindau, Germany

- 2011 - 2014: Stipend of the Excellence Foundation for the Promotion of the Max Planck Society.

- 2012 Djordje Stefanovic Plaque for the Best Graduated Student in the Department of Biochemistry, Faculty of Chemistry, University of Belgrade.

- 2011 Serbian Chemical Society Award: the Best Graduated Student in the Academic 2011.

- 2010 Institute of International Education: Scholarship for the best student of Serbia awarded in collaboration with the Eurobank EFG.

- 2010 British council/IAESTE: Global Work Experience Scholarship for a research project at the University of Edinburgh.

- Ministry of Science and Education, Republic of Serbia Stipend for the academic years 2007/8, 2009/10, 2010/11.

- 2009 - Outstanding oral presentation award, Research Scholar Forum, Indiana, PA, USA.

- 2009 - Dean's List at the Indiana University of Pennsylvania, Indiana, PA.

- US Government and World Learning Stipend for the academic studies 2008 - 2009 at the Indiana University of Pennsylvania, USA. 
- American Chemical Society and the Department of Chemistry at Indiana University of Pennsylvania annual award for student achievement in the academic year 2008/9.

- 2007 Diploma for the Best student in the Gymnasium, Fifth Belgrade Gymnasium.

\section{SCIENCE AND COMMUNITY}

2014 - present: Coordinator of the PhD/Postdoc Community at the Max Planck Institute for Biophysical Chemistry, Göttingen, Germany. *Representing around $200 \mathrm{PhD}$ students and postdocs, organizing Scientific Seminars (hosted more than ten professors from different countries/institutions), Career Seminars (hosted journal editors, company CEOs), Tea Time Talk (I am particularly proud to design this event which is a session that brings two junior group leaders sharing their path to success).

2014: Head of the Speakers Group (team-chair, coordination and executive work); International conference: $11^{\text {th }}$ Horizons in Molecular Biology (approx. 300 participants)

2013 - 2014: PhD General Student Representative at the Göttingen Graduate School for Biophysics, Neuroscience and Molecular Biosciences (encompasses 12 graduate programs over major natural science disciplines with more than 500 students).

TEACHING (during graduate studies)

February 2015 - April 2015: Supervising a guest student from Windber Research Institute, Windber, PA USA

January 2014 - April 2014: Supervisor of two master students from IMPRS Molecular Biology and IMPRS Neuroscience Programs, University of Göttingen.

May 2014 - June 2014: XLAB Lectures at the International Science Camp

2012 - 2014: Laboratory Course: In vitro Reconstitution of the SNARE mediated vesicle fusion, offered each semester, Göttingen Graduate School for Neuroscience, Biophysics and Molecular Biosciences, University of Götitngen.

2012 - 2014: Tutor in the International Max Planck Research School in Molecular Biology for the following topics/lectures: Biological Membranes, Protein Sorting and Processing, Membrane Traffic. 


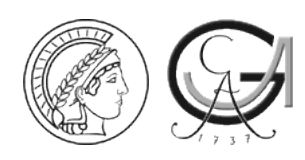

\section{LAB PROJECTS DONE BEFORE STARTING DOCTORAL WORK}

- $\quad 7 / 2011$ - 9/2011 Analysis of the Ebf1 interaction partners using the proximity ligation assay, Department of Cellular and Molecular Immunology, Max Planck Institute for Immunobiology and Epigenetics, Freiburg, Germany

- 10/2010 - 6/2011 Variability of mitochondrial DNA haplotypes in Drosophila subobscura, Department of the Population Genetics, Institute for Biological Research, Belgrade, Serbia

- 7/2010 - 9/2010 Racemization studies of recombinant N-acetyl amino acid racemase from Geobacillus kaustophilus and Geobacillus thermodenitrificans, School of Chemistry, The University of Edinburgh, Edinburgh, UK

- 7/2009 - 9/2009 The cross correlated relaxation rate as a tool to investigate protein dynamics, Department of NMR-based Structural Biology, Max Planck Institute for Biophysical Chemistry, Göttingen, Germany

- 9/2008 - 5/2009 The Srs2 protein expression using the pET11c expression vector in the BL21 (DE3) bacterial protein expression strain of Escherichia coli, Department of Biochemistry, Indiana University of Pennsylvania, Indiana, USA

- 9/2008 - 5/2009 Consequence of the IP3 protein receptor absence on calcium ion influx and circadian cycle of Paramecium, Department of Biology, Indiana University of Pennsylvania, Indiana, USA

- 5/2007 - 12/2007 Antimutagenic effect of marigold, plantain, and stinging nettle tea in a somatic mutation and recombination test on Drosophila melanogaster, Department of the Population Genetics, Institute for Biological Research, Belgrade, Serbia

\section{HOBBYS}

Photography, debate clubs, improvisational theater, hiking and running.

\section{LANGUAGE SKILLS}

Serbian: native;

English: proficiency level (C2);

German: advanced level (C1);

French: intermediate level (B1). 


\subsection{LIST of PUBLICATIONS}

\section{Publications during graduate studies}

Milovanovic D, Platen M, Junius M, Diederichsen U, Schaap I, Honigmann A, Hell SW, Jahn R, van den Bogaart G. Calcium induces syntaxin 1 mesoscale domain formation through phosphatidylinositol 4,5-bisphosphate. manuscript in preparation.

Milovanovic D, Honigmann A, Risselada JH, Pähler G, Müllar S, Grubmüller H, Janshoff A, Diedrichsen U, Eggeling C, Hell SW, van den Bogaart G, Jahn R. Hydrophobic mismatch sorts SNAREs in distinct plasma membrane domains. (2015) Nature Communications, 6: 5984

Milovanovic D, Jahn R. Organization and dynamics of SNARE proteins in the presynaptic membrane. (2015) Frontiers in Physiology, 6: 89

Schwenen LL, Hubrich R, Milovanovic D, Geil B, Yang J, Kros A, Jahn R, Steinem C. Resolving single membrane fusion events on planar porespanning membranes. (2015) Scientific Reports, 5: 12006

Honigmann A, van den Bogaart G, Iraheta E, Risselada JH, Milovanovic D, Pena V, Mueller, Muellar S, Meyenberg K, Grubmüller H, Diederichsen U, Fasshauer D, Hell SW, Eggeling C, Jahn R. Phosphatidylinositol 4,5bisphosphate clusters act as molecular beacons for vesicle recruitment. (2013) Nature Structural \& Molecular Biology, 20: 679-86.

Sadek M, Berndt D, Milovanovic D, Jahn R, Diederichsen U. Nucleobasefunctionalized $\beta$ peptide as a modified model of a SNARE protein for membrane fusion. manuscript in preparation.

Publications prior to graduate studies

Jelic M, Castro J, Kurbalija Novicic Z, Kenig B, Dimitrijevic D, Savic Veselinovic M, Jovanovic M, Milovanovic D, Stamenkovic Radak M, Andjelkovic M. Absence of linkage disequilibria between chromosomal arrangements and mtDNA haplotypes in natural populations of Drosophila subobscura. (2012) Genome, 55: 214-21.

Fenwick RB, Esteban-Martín S, Richter B, Lee D, Walter KFA, Milovanovic D, Becker S, Lakomek N, Griesinger C, Salvatella X. Weak long-range correlated motions in a surface patch of ubiquitin involved in molecular recognition. (2011) Journal of the American Chemical Society, 133: 10336-9. 